\title{
Development and validation of a model for the response of the Belle II vertex detector
}

\author{
Dissertation \\ zur Erlangung des mathematisch-naturwissenschaftlichen Doktorgrades \\ "Doktor rerum naturalium" \\ der Georg-August-Universität Göttingen \\ im Promotionsprogramm ProPhys \\ der Georg-August University School of Science (GAUSS)
}

\author{
vorgelegt von \\ Benjamin Schwenker \\ aus Rosenheim
}

Göttingen, 2014 


\section{Betreuungsausschuss}

Prof. Dr. A. Frey, II. Physikalisches Institut, Georg-August-Universität Göttingen

Prof. Dr. A. Quadt, II. Physikalisches Institut, Georg-August-Universität Göttingen

PD Dr. J. Große-Knetter, II. Physikalisches Institut, Georg-August-Universität Göttingen

\section{Mitglieder der Prüfungskommission}

Referentin: Prof. Dr. A. Frey, II. Physikalisches Institut, Georg-August-Universität Göttingen

Korreferent: Prof. Dr. A. Quadt, II. Physikalisches Institut, Georg-August-Universität Göttingen

\section{Weitere Mitglieder der Prüfungskommission}

PD Dr. J. Große-Knetter, II. Physikalisches Institut, Georg-August-Universität Göttingen

Prof. L. Covi, PhD, Institut für Theoretische Physik, Georg-August-Universität Göttingen

Jun.-Prof. Dr. S. Schumann, II. Physikalisches Institut, Georg-August-Universität Göttingen

Prof. Dr. W. Kollatschny, Institut für Astrophysik, Georg-August-Universität Göttingen

Tag der mündlichen Prüfung: 16.10.2014

Referenz: II.Physik-UniGö-Diss-2014/05 



\section{Abstract}

The future super flavour factory SuperKEKB with its detector system Belle II offers precision physics measurements to test the Standard Model or probe undiscovered phenomena. The physics goals of Belle II require a very precise detection of the decay point, or vertex, of $B$ mesons from their low momentum decay products. A novel, two layer vertex detector composed of very thin, high resolution silicon pixel detectors based on Depleted Field Effect Transistors (DEPFET) is in production for Belle II. A realistic and experimentally validated simulation for DEPFET pixel detectors is a crucial tool to optimize the resolution of the vertex detector well before construction. In this thesis, a detailed detector simulation for the response of thin DEPFET pixel detectors to charged particles is presented. The detector simulation provides a description of the straggling of particles in silicon, the drift, diffusion and collection of the signal and the response of the read-out electronics. The model yields a precise prediction of the spatial resolution of the detector, given design parameters such as the pixel size, the sensor thickness and the electronics noise. The second part of this thesis is devoted to the results of measurements of the response of DEPFET detector prototypes to a beam of charged particles in test beam lines at CERN and DESY. New methods for the calibration, tracking and alignment of the EUDET/AIDA beam telescope are presented, that allow a quantitative determination of the signal distribution, hit efficiency and spatial resolution. Comparison of the results with the predictions of the response model yields good agreement. The validated model predicts a spatial resolution of $\sim 10 \mu \mathrm{m}$ for $50 \mu \mathrm{m}$ thick DEPFET sensors for Belle II, satisfying the vertex detector requirements. 


\section{Contents}

1. Introduction: Motivation and overview of study 1

2. Vertexing at Belle II: Framework and scope of research 4

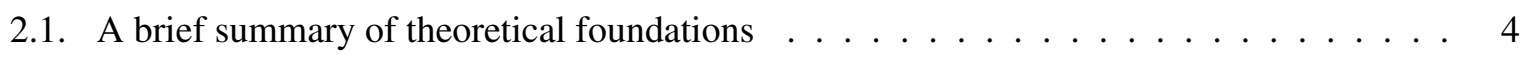

2.1.1. Introduction to particles and interactions $\ldots \ldots \ldots \ldots$. . . . . . 4

2.1.2. Cornerstones of the Standard Model . . . . . . . . . . . . . . . . . . . 5

2.1.3. CP violation in the Standard Model . . . . . . . . . . . . . . . . . . 7

2.2. Belle II at SuperKEKB: Challenges and opportunities f . . . . . . . . . . . 8

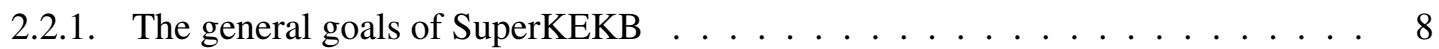

2.2 .2 . Vertexing challenges at Belle II . . . . . . . . . . . . . . . . . 10

2.2.3. The DEPFET pixel detector . . . . . . . . . . . . . . . . . . . . 12

2.3. Scope and design of research: Three steps towards a valid detector simulation . . . . . . 13

2.3.1. First step: Simulation of a vertex detector module . . . . . . . . . . . . . . . . 14

2.3.2. Second step: Data analysis with EUDET beam telescope . . . . . . . . . . . . . 14

2.3.3. Third step: Validation of simulation results $\ldots \ldots \ldots \ldots$

3. The DEPFET detector simulation: Algorithm and its implementation 17

3.1. Introduction: Overview of simulation and its challenges . . . . . . . . . . 17

3.2. The software framework: Tools and methods for data simulation and reconstruction. . . 19

3.2.1. Key components of the ILCSoft framework . . . . . . . . . . . . . . . . . . 19

3.2.2. The geometry model for a beam telescope . . . . . . . . . . . 20

3.2 .3 . Event data model: energy losses, digits, clusters and hits . . . . . . . . . . 23

3.3. The DEPFET simulation algorithm: A three step approach . . . . . . . . . . . 25

3.3.1. Step 1: From energy losses to signal charge deposition . . . . . . . . . . . 26

3.3.2. Step 2: Collection of signal charge in DEPFET pixels . . . . . . . . . . . 27

3.3.2.1. Signal detection with DEPFET pixel sensor . . . . . . . . . . 27

3.3.2.2. Fast model for drift and diffusion in DEPFET pixels . . . . . . . . . 28

3.3.2.3. Fast vertical signal charge collection . . . . . . . . . . . . 30

3.3.2.4. Lateral charge collection between pixels . . . . . . . . . . . 32

3.3.3. Step 3: From collected signal charge to readout digits $\ldots \ldots \ldots \ldots$

3.4. A case study on spatial resolution of thin DEPFET sensors $\ldots \ldots \ldots$. . . . . . 36

3.4.1. The setup of the simulation: Overview of parameters . . . . . . . . . . . . . . . . . . . . . 36

3.4 .2 . Steps for data reconstruction . . . . . . . . . . . . . . 37

3.4.2.1. From readout digits to clusters . . . . . . . . . . . . . 37

3.4.2.2. From clusters to local hit positions . . . . . . . . . . . . . . . . 39

$3.4 .2 .3 . \quad$ Sampling hit position errors $\ldots \ldots \ldots . \ldots . \ldots . \ldots 39$ 
3.4.3. The results: cluster shapes and spatial resolution . . . . . . . . . . . . . . 40

4. The reconstruction of beam telescope data: methods, steps and calibration 44

4.1. Overview of reconstruction and its challenges . . . . . . . . . . . . . . . . 44

4.2. The reconstruction framework: From digits to tracks $\ldots \ldots \ldots$. . . . . . . 45

4.2.1. High energy particle beams at DESY and CERN . . . . . . . . . . . . 45

4.2.2. The EUDET beam telescope as data source . . . . . . . . . . . . . . . 48

4.3. The reconstruction of clusters and hits (Step 1) $\ldots \ldots \ldots$. . . . . . . . . . . 49

4.4. Track finding and fitting (Step 2) . . . . . . . . . . . . . . . . . 52

4.4.1. A fast and combinatorial track finder . . . . . . . . . . . . . . 52

4.4.2. Track fitting with Kalman Filters . . . . . . . . . . . . . . . . . . . 53

4.4.2.1. Local track parameters and residuals . . . . . . . . . . . . . . 54

4.4.2.2. A model for track parameters in collimated particle beams . . . . . . . 55

4.4.2.3. Multiple scattering and estimation of the radiation length . . . . . . . 59

4.5. The telescope alignment (Step 3) $\ldots \ldots \ldots$. . . . . . . . . . . . 60

4.5.1. Goals and problems of telescope alignment . . . . . . . . . . . . . . . 60

4.5.2. The parametrization of telescope misalignment . . . . . . . . . . . . . . 62

4.5.3. Survey measurements and initial telescope misalignment . . . . . . . . . . . 63

4.5.4. A new approach to better telescope alignment . . . . . . . . . . . . . 65

4.5.4.1. Pre-Alignment: Beam spot correction and hit correlations . . . . . . . 65

4.5.4.2. Alignment with tracks: Kalman Alignment Algorithm with annealing 68

4.5.4.3. Strategy for telescope alignment and control plots . . . . . . . . . 70

5. Thin DEPFET modules in test beams: Validation of the simulation 72

5.1. Introduction to the validation approach and the results $\ldots \ldots \ldots \ldots$. . . . . 72

5.2. Overview of prototype sensors and the test system . . . . . . . . . . . . 73

5.3. Offline calibration of sensor raw data . . . . . . . . . . . . . . . . . . . 75

5.4. Results of the validation measurements . . . . . . . . . . . . . . . . . . . . . 79

5.4.1. Study of signal and cluster size distributions $\ldots \ldots \ldots$. . . . . . . . 80

5.4.2. Study of the intrinsic spatial resolution . . . . . . . . . . . . . . 81

5.4.3. Study of hit detection efficiency . . . . . . . . . . . . . . . . . 84

5.4.4. Study of in-pixel charge collection . . . . . . . . . . . . . . . . . 87

$5.4 .5 . \quad$ Study of in-pixel charge sharing . . . . . . . . . . . . . . . . . . . 89

6. Summary and outlook 93

A. Track fitting in the EUDET telescope with Kalman Filters 95

A.1. A track model for the EUDET beam telescope . . . . . . . . . . . . . . . . . . 95

A.2. Kalman Filters for track fitting . . . . . . . . . . . . . . . . . . . 100

A.2.1. Linearization for tracks in a collimated beam . . . . . . . . . . . . . . . 100

A.2.2. Forward, backward and time reversed filters . . . . . . . . . . . . . 101

A.2.3. Track fitting with forward and backward filters . . . . . . . . . . . . . 102

A.3. Validation of track fitting with pseudo experiments . . . . . . . . . . . . 103 
B. Kalman Alignment Algorithm with annealing 105

B.1. The sequential update rule . . . . . . . . . . . . . . . . . . . . 105

B.2. The initial alignment covariance and annealing . . . . . . . . . . . . . . 107

B.3. Validation of telescope alignment $\ldots \ldots \ldots \ldots$. . . . . . . . . . . . . .

C. GEAR interface for test beams 113 


\section{Introduction: Motivation and overview of study}

Experiments at particle colliders developed into a prototype experiment of modern particle physics in the second half of the 20 th and beginning 21 th century. Starting from a seemingly simple situation, a head on collision of a single electron and positron in vacuum, nature reveals some of her deepest secrets under well controlled laboratory conditions. The experimental results of collider experiments provide an important test for the principles of quantum mechanics and special relativity. Both quantum mechanical interference and the equivalence of mass and energy of special relativity were demonstrated at subatomic scales with high accuracy. Moreover, the experimental data showed the unification of phenomena as different as electromagnetism, radioactivity and nuclear physics into an elegant theoretical framework called the Standard Model of Particle Physics (SM).

The Standard Model lists the fundamental particles that have been found so far: the three families of quarks and leptons, and the four bosons responsible for the fundamental forces of nature excluding gravity. Even more, the ingredients of the Standard Model - quantum electrodynamics, quantum chromodynamics and the electroweak theory - precisely describe the interactions of all the particles in agreement with all available measurements. In the Standard Model the Higgs mechanism [1, 2] proposed in 1964 is responsible for breaking the electroweak symmetry, giving mass to the $W^{ \pm}$and $Z$ gauge bosons and the elementary constituents of matter. The discovery of a new particle with properties compatible with that of the Higgs boson is a historic success of the Standard Model [3, 4].

The Standard Model has three families of quarks and leptons distinguished by their flavour, the charge of the weak interaction. The mixing of flavours due to charged-current interactions and the violation of the combined Charge-Parity (CP) symmetry of the weak interaction are described by the CabbiboKobayashi-Maskawa formalism [5]. The study of CP violation may prove the key to understanding the dominance of matter over anti-matter in our universe, even if in our current understanding the CP violation in the SM is too small to explain the excess of matter [6, 7]. The experiments BaBar [8] and Belle [9] at the $B$ factories (asymmetric $e^{+} e^{-}$colliders running at the $Y(4 s)$ resonance) empirically established the CKM model of quark mixing and $\mathrm{CP}$ violation.

To further explore CP violation in nature, the super flavour factory SuperKEKB will go into operation at KEK in 2016 [10]. This asymmetric $e^{+} e^{-}$accelerator is designed to operate with energies of $4 \mathrm{GeV} / 7 \mathrm{GeV}$ per beam and its nominal instantaneous luminosity of $8 \times 10^{35} \mathrm{~cm}^{-2} \mathrm{~s}^{-1}$ is about a factor 40 higher compared to the previous machine KEKB. A new detector, Belle II as an upgrade of Belle, is required to deal with the increased interaction and background rates. The Belle II detector [11] is a hermetic detector system which allows the reconstruction of particle tracks after a collision, and performs the measurement of energy, momentum and charge of the produced particles. The precise measurement of the decay origin or vertex of $B$ mesons produced in collisions at SuperKEKB require a pixel vertex detector (PXD) placed in close proximity to the interaction point, which can deal with a 


\section{Introduction: Motivation and overview of study}

high track multiplicity, perform well in an environment with high radiation levels, and offers an intrinsic position resolution in the order of $10 \mu \mathrm{m}$. By keeping the material budget of the vertex detector very low, Coulomb scattering of low momentum particles is strongly reduced and the decay vertices are reconstructed with superior precision. A new addition to the Belle II detector suited for this is a two layer silicon pixel detector based on the DEPFET technology.

The concept of particle detection with fully Depleted Field Effect Transistors (DEPFET) was proposed by Kemmer and Lutz in 1987 [12]. Excellent signal over noise performance is provided by integration of a MOSFET (Metal Oxide Semiconductor Transistor) in each pixel, as the first amplification stage of readout electronics. Thinning technologies make it possible to manufacture DEPFET sensors with thickness of active sensor area $50 \mu \mathrm{m}$ [13]. A segmentation of the sensor in a matrix of DEPFET pixels with a typical pitch of $50-75 \mu \mathrm{m}$ offers an intrinsic spatial resolution of $\sim 10 \mu \mathrm{m}$ suitable for the Belle II case. New application specific integrated circuit (ASIC) readout chips allow to readout a DEPFET detector with about 8 million pixels surrounding the collision point in $20 \mu$ s with low readout noise. The resolution of the vertex detector depends strongly on two questions that can only be studied in simulation studies before construction: What is the density of background hits in the PXD for an integration time of $20 \mu$ s proposed for the PXD? Secondly, what is the intrinsic spatial resolution for the position resolved detection of charged tracks using the proposed design of thin DEPFET detectors?

Performance studies for the pixel vertex detector strongly depend on an accurate simulation of the DEPFET response to a single charged particle hitting a module of the vertex detector, which is the scope of this thesis. A detailed simulation of the DEPFET response is required to predict the detected energy loss per pixel for a charged particle traversing the pixel matrix at precisely controlled conditions: momentum, angle of incidence and in-pixel hit position. In this thesis, a fast simulation algorithm for the DEPFET response is presented, taking into account the particle interactions with the sensor material responsible for the signal generation, the analog signal amplification in the DEPFET transistor and the analog-to-digital conversion in the drain current digitizer (DCD) chip. A first implementation of the simulation algorithm in the ILCSoft computing framework was presented in 2011 [14] and used for optimization studies for the design of the Belle II vertex detector [11]. One of the contributions of this thesis is the implementation of a physical model for drift and diffusion of signal charge in the DEPFET pixels. It allows an improved estimation for cluster shapes and spatial resolution of the DEPFET pixel detectors while keeping the simulation time low.

In order to validate the DEPFET simulation algorithm, the second part of the research presented in this thesis deals with the development of a software framework providing precise tools for the reconstruction of beam telescope data. The latest prototype modules tested in 2012 and 2013 are a big step towards the final specifications for Belle II modules. The tested sensors are $50 \mu \mathrm{m}$ thick and have pixel cell dimensions of $50 \times 75 \mu \mathrm{m}^{2}$. The test system consists of a small matrix with $32 \times 64$ pixels. The readout of the test system uses first production versions of DCDB and Switcher chips [15, 16] developed for the Belle II pixel detector. All steps in the simulation of DEPFET response have a real counterpart in the test system. For this reason, a detailed validation of the simulation algorithm is feasible for the first time.

In order to compare real data with simulations, precise tools for an independent prediction of the particle momentum, in-pixel hit position and angle of incidence at the DEPFET pixel matrix, the so-called local track parameters, are needed. The validation study is based on the operation of DEPFET modules as a device under test inside the EUDET beam telescope [17]. The EUDET beam telescope is a precise 


\section{Introduction: Motivation and overview of study}

tracking detector installed at intense sources of charged particles with well known momentum; a high energy beam line. A precise calibration and analysis of beam telescope data allows to achieve a high resolution for local track parameters at the device under test in the order of a few micrometers for positions and few milliradians for incidence angles. In this thesis, new methods for reconstruction and calibration of telescope data were implemented and tested in the ILCSoft framework. The methodical approach followed here consists of three steps to beam telescope data reconstruction. The first step, clustering, is the estimation of the local hit position from sensor raw data. For clustering, we present methods for an improved data driven estimation of spatial resolution for telescope detectors. For the second step, tracking, the key point is the implementation of a Kalman Filter based local track parameter estimation. The method features an optimal weighting of telescope hits taking into account Coulomb scattering at telescope detectors and air and allows free $3 \mathrm{D}$ rotations of all telescope parts. The last step, telescope alignment, is the data driven software correction of the geometrical positions of telescope detectors. An extended implementation of the Kalman Alignment Algorithm proposed in [18] is presented here that allows to take into account all three rotations and shifts of all detector modules in the beam telescope. A new method to correct global distortions of the telescope, the so-called shearing and torsion, is presented that uses an explicit mathematical model of the particle beam as an additional constraint for alignment. The performance of Kalman Filter based track fitting and telescope alignment methods is tested in detailed pseudo experiments.

The validation framework developed provides a means for a detailed testing of all parts of the DEPFET response simulation. The simulation of energy loss straggling and the effect of delta electrons on hit reconstruction are studied. Prototypes with $50 \mu \mathrm{m}$ thin sensors achieve the designed signal amplification and provide a comfortable signal to noise ratio of $20-30$ for position resolved hit detection. The intrinsic spatial resolution is studied at high $(120 \mathrm{GeV})$ and low momentum $(1-6 \mathrm{GeV})$ particle beams as a function of the track incidence angle. Very large data sets with several million precisely reconstructed tracks hitting the sensor allow to study the charge sharing between neighbouring pixels depending on the in-pixel hit position. Charge sharing, the division of signal charge between pixels around the hit, is used as a proxy for the net effect for the drift and diffusion of signal electrons in the pixel sensor.

Following the above described research design, this thesis is organized as follows: Chapter 2 outlines the challenges and opportunities of vertexing at Belle II motivating the need for simulations and introduces the EUDET beam telescope. Chapter 3 presents the DEPFET response simulation and the first implementation of the algorithm in ILCSoft. The following chapter 4 introduces the methods used for analysis of beam telescope data. The performance of new analysis methods is studied on data from real test beam experiments. Chapter 5 presents the validation study for the DEPFET response simulation with DEPFET modules in the EUDET beam telescope. 


\section{Vertexing at Belle II: Framework and scope of research}

The Standard Model of Particle Physics is currently the most complete and most thoroughly tested theory for the basic constituents of matter and their behaviour. Despite its big success at earth bound experiments in high energy physics, the Standard Model is in disagreement with cosmological observations. The Standard Model predicts a far too small violation of the CP symmetry in physical processes and cannot explain the excess of baryonic matter over antimatter. These open questions motivate the construction of the super flavour factory SuperKEKB at Tsukuba, Japan, for improved measurements of $\mathrm{CP}$ violation in the context of the Standard Model. SuperKEKB and its detector system Belle II have the potential to reveal new sources of CP violation and to constrain physics beyond the Standard Model.

As already outlined in the introduction, the aim of this thesis is to develop and validate a simulation for the DEPFET pixel vertex detector at Belle II. To create a sound understanding of the challenges of this pixel detector as input for the simulation, this chapter is structured as follows: based on a brief summary of the Standard Model of Particle Physics and the current measurements of CP violation in the context of the CKM model for quark flavour maxing, the second part outlines the experimental opportunities and challenges for precision measurements of CP violation at Belle II. The requirements for the pixel vertex detector at Belle II and the need for reliable detector simulations are motivated. The last part of the chapter gives an overview of the research program presented in this thesis.

\subsection{A brief summary of theoretical foundations}

\subsubsection{Introduction to particles and interactions}

The discovery of the first elementary particles, electrons and photons, dates back to the beginning of the 20 th century. Early experiments showed that electrons can be ripped off from the atomic shell and placed on small oil drops between plate condensers [19]. The electric charge of the oil drops was always found to be an integer multiple of a fundamental unit depending on the number of electrons per oil drop. All electrons carry precisely the same electric charge and there is no measurable difference between one electron and another. These properties of electrons apply quite generally to the other elementary particles discovered so far: they can be localized in space and time, carry discrete charges and come in indistinguishable species. Unlike the electrons, many elementary particles are unstable and require more advanced experimental detectors for their observation from stable decay products.

The existence of the photon was postulated in 1905 by Einstein as an explanation of the photoelectric effect [20, 21]. The photoelectric effect is the observation that many metals emit electrons when ultraviolet light shines upon them. Einstein realized that the emission of electrons occurs one by one at different times and localized positions on the metal surface. The emission of an electron requires an 
amount of energy and momentum that arrives from an ultraviolet light source in discrete packets called photons. More precisely, the observed process consists of the emission of a photon at the light source, the flight, or better, propagation of the photon to the metal and finally the absorption of the photon by an electron in the metal. On arrival at the metal, the photon instantaneously transfers its energy and momentum to an electron and vanishes from space and time. It is neither possible that the photon splits in two on the way to the plate nor can the electron absorb only half a photon. In the language of modern particle physics, the photoelectric effect is the first example for a local quantum interaction between an electron and a photon. Quantum interactions involve the creation and destruction of elementary particles at a point in space and time called interaction vertex. More generally, all elementary particles carrying electric charge have a probability to spontaneously emit or absorb a photon.

A precise theory for the interactions of electrons and photons in relativistic space time, called Quantum Electrodynamics (QED), was developed by Feynman, Tomonaga and Schwinger in the late 1940's [22, 23, 24]. As a quantum theory, QED predicts an interaction probability for any possible experimental measurement as the square of a quantum amplitude. In Feynman's new formulation of QED, there are only three basic amplitudes from which all others can be computed. The first two amplitudes describe the propagation of an electron or a photon from one point in space time to another. The third amplitude describes the local emission, or absorption, of a photon by an electron. QED is the first theory that correctly anticipates the possibility for the propagation of electrons backward in time. In a real experiment where time runs forward, an electron moving backward in time appears as having the wrong, more precisely, opposite electric charge. These wrong sign electrons are called positrons and are the first example of anti-particles.

QED is an extremely successful theory capable to describe all physical processes in the atomic shell and modern optics with very high precision [25]. However, QED fails to account for physical processes inside the atomic nucleus. The nucleus itself cannot consist of electrons or positrons and a theory beyond QED was needed to understand its constituents and behaviour. The rapid progress in particles physics in the last 50 years, both experimental and theoretical, brought us definite answers. The protons and neutrons inside atomic nucleus are just the lightest examples for a type of matter called baryons which consist of triplets of new elementary particles called quarks.

\subsubsection{Cornerstones of the Standard Model}

Today, the Standard Model of Particle Physics (SM) is a theory that contains nearly everything we know about elementary particles and their interactions. This section aims to briefly outline the main ingredients of the Standard Model. More detailed descriptions of the SM can be found in various text books [26, 27].

Fig. 2.1 shows a representation of the known elementary particles in the SM. All elementary particles carry a quantum mechanical angular momentum called spin. Like the electric charge, the spin is quantized and can be measured in units of Planck's constant $\hbar$. The fermions - leptons and quarks are experimentally found to be spin $\frac{1}{2}$ particles. The interactions between fermions are described by the exchange of spin 1 gauge bosons: the electromagnetic force is carried by photons $(\gamma)$, the weak force by $W^{ \pm}$and $Z^{0}$ bosons and the strong force by gluons $(g)$. While photons and gluons are thought to be massless, $W^{ \pm}$and $Z^{0}$ bosons are massive. Only particles which are electrically charged couple to photons; they can spontaneously absorb or emit photons in quantum mechanical interactions. The photon 


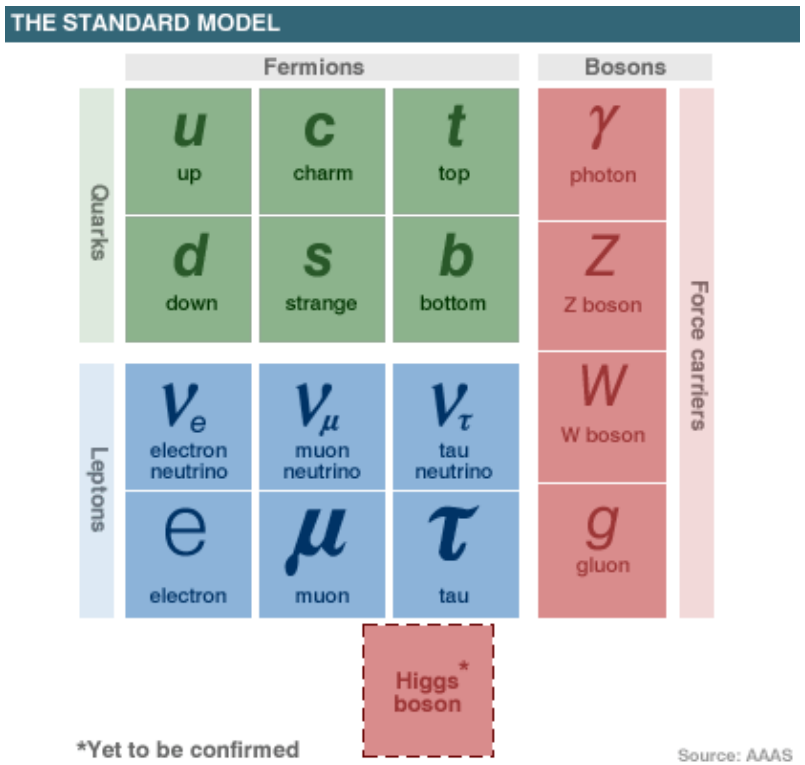

Figure 2.1.: Known elementary particles in the SM: there are three generations of quarks and leptons. The interactions are mediated by four types of gauge bosons. The recently discovered Higgs boson is shown at the bottom.

itself carries no electric charge and cannot directly couple to other photons. Colour charge, however, is only carried by quarks and gluons, which hence interact via the strong force. All fermions carry a weak charge and couple to $W^{ \pm}$and $Z^{0}$ bosons. For example, the spontaneous emission of a $W^{ \pm}$from a down quark in the neutron is the reason for radioactive $\beta$ decays. The form of the different couplings between fermions and gauge bosons are entirely determined by the underlying local gauge symmetries in the SM.

Leptons and quarks exist in three generations, where the masses of the particles increase from generation to generation. Additionally, for every fermion, there is an antiparticle with the same properties like the particle, but with opposite values of the additive quantum numbers, such as electric charge and the third component of the weak isospin, $I_{3}$.

Each quark generation consists of an up-type quark with $I_{3}=+\frac{1}{2}$ and its down-type quark partner with $I_{3}=-\frac{1}{2}$. Due to the parity violating nature of the weak force, only left-handed quarks form doublets of up- and down-type quarks, while right-handed quarks form singlets. The quark doublets read: up $(u)$ and down $(d)$ quark, charm $(c)$ and strange $(s)$ quark, and top $(t)$ and bottom $(b)$ quark. Up-type quarks have an electric charge $+\frac{2}{3} e$, the charge of the down-type quarks is $-\frac{1}{3} e$.

The lepton doublets consist of a lepton with electric charge $-e$ (electron $e$, muon $\mu$, tau $\tau$ ) and the corresponding neutrino $\left(\nu_{e}, \nu_{\mu}, \nu_{\tau}\right)$, which is electrically neutral. The charged leptons form right-handed singlets. Since neutrinos are assumed to be massless in the SM, no right handed neutrino singlets are foreseen in the SM. In neutrino oscillation experiments, however, it was shown that neutrinos have nonvanishing masses [28]. Although the neutrino masses have not yet been measured, the differences in the squares of their masses were measured in the oscillation experiments. However, the neutrino masses must be very small compared to the scales present in high energy physics experiments and can hence be ignored in this context.

Mathematically, the SM is formulated as a renormalizable, Lorentz invariant and local gauge invariant quantum field theory. Interactions are introduced by local gauge symmetries. The structure of the gauge 
groups is $S U_{C}(3) \times S U_{L}(2) \times U_{Y}(1)$, where $S U_{C}(3)$ is the gauge group for Quantum Chromodynamics (QCD) [29, 30, 31], which describes the strong interaction, and $S U_{L}(2) \times U_{Y}(1)$ is the gauge group for the unified electromagnetic and weak interactions [32, 33, 34]. The symmetry between electromagnetic and weak interactions is spontaneously broken via the Higgs mechanism [1, 2]. The Higgs mechanism describes the generation of particle masses in the SM. It implies the existence of at least one additional spin 0 boson called Higgs particle with defined couplings to fermions and vector bosons. In 2012, a new Higgs like spin 0 boson was indeed discovered at the ATLAS and CMS experiments [3, 4].

Similar to QED in the late 1940's, the SM is known to have a number of serious shortcomings. Precision measurements of the cosmic microwave background show that only $5 \%$ of all energy in the universe can be attributed to known particles. The known $5 \%$ of matter are dominated by hadrons, particles composed of either a quark anti-quark pair (mesons) or a triplet of quarks (baryons). The composition of the remaining $95 \%$ of energy in the universe are $27 \%$ dark matter and $68 \%$ dark energy. However, even $5 \%$ of hadronic matter is much higher than could be expected from our existing theories which stipulate nearly all baryonic matter and antimatter should have been annihilated just after the big bang. A theoretical work by Sakharov showed that $\mathrm{CP}$ violation is an essential ingredient to understanding the observed surplus of matter over antimatter in the universe [6].

\subsubsection{CP violation in the Standard Model}

The first experimental observation of CP violation in nature was achieved in the neutral Kaon system by Cronin and Fitch in 1964 [35]. In 1973, Kobayashi and Maskawa proposed a theory describing the CP violation in the SM by a mixing of the three quark generations in weak interactions [5]. All down-type quarks have a mixing amplitude to spontaneously decay into up-type quarks by the emission of a $W^{-}$ boson. To explain CP violation in the SM, Kobayashi and Maskawa were lead to predict the existence of a third quark generation years before the top and bottom quarks, the members of the third quark generation, were experimentally discovered. Moreover, the KM theory predicted that the nine quark mixing amplitudes cannot be independent but form what is today called the CKM matrix, a unitary $3 \times 3$ matrix with one complex phase responsible for $\mathrm{CP}$ violation.

The unitarity constraints of the CKM matrix can be displayed by six triangles in the complex plane, whose area is non vanishing in the case of CP violation. The unitary triangle (UT) with the largest angles and biggest $\mathrm{CP}$ violation was predicted to occur for the mixing of the bottom $(b)$ quark into the three up-type quarks $(u, c, t)$. Two dedicated experiments, BaBar at the PEP-II collider and Belle at the KEKB collider, were built to study the CP violation in the UT from decays of $B$ mesons produced in electron positron collisions. $B$ mesons are hadrons and contain a heavy bottom quark (either $b$ or $\bar{b}$ ) together with a light up $(u)$ or down $(d)$ quark. The goal of the $B$ factories BaBar and Belle was to overconstrain the UT from independent measurements of all sides and angles of the UT. A global fit of the unitary triangle is reproduced here as Fig.2.2. It shows that the measured sides and angles of the UT indeed form a triangle within the current statistical and systematic errors. The experimental results from BaBar and Belle were of high importance for the confirmation of the CKM mechanism and the two experiments were mentioned when the Nobel prize in physics 2008 was finally awarded to Kobayashi and Maskawa for the invention of the CKM mechanism.

So far, the CKM matrix is the only source of CP violation in the SM. But cosmological models suggest that the known CP violation in the SM is far too small to explain the observed surplus of matter over 


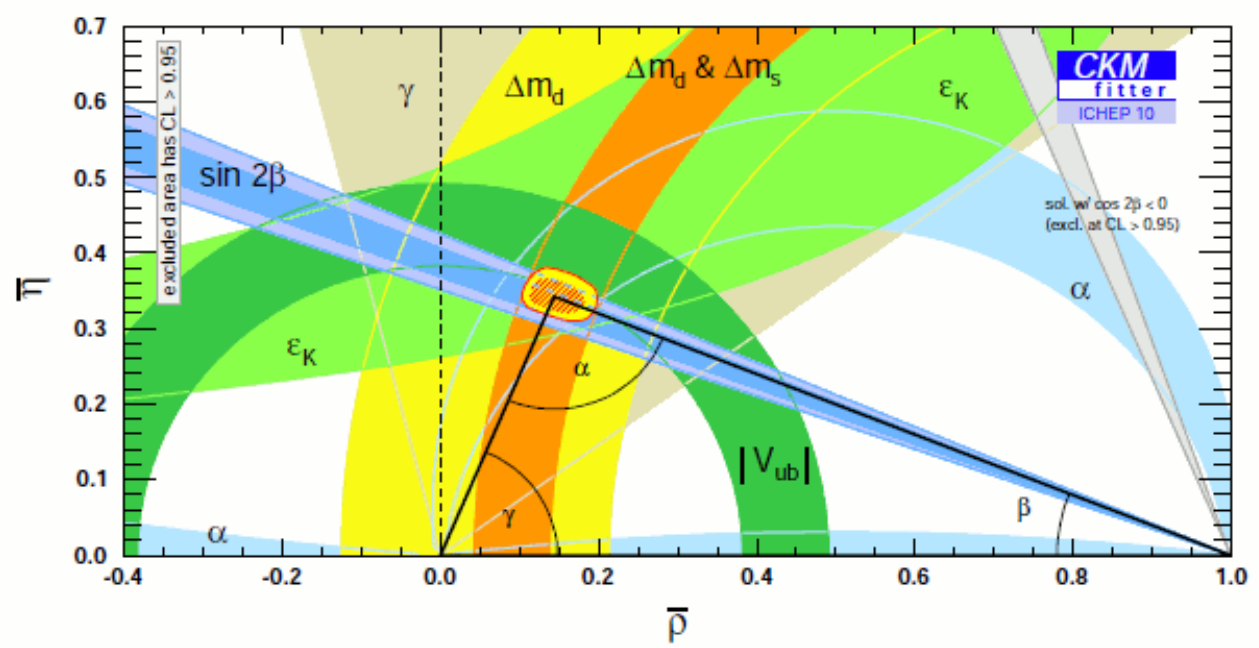

Figure 2.2.: Confidence levels of mixing angles of the CKM matrix for the global CKM fit. The shaded areas are regions of $5 \% \mathrm{CL}$. The hatched area in the center of the combined fit indicates the region were systematic errors dominate [36].

antimatter in the universe [7]. In other words, there must be undiscovered sources for CP violations beyond the Standard Model. Upgraded $B$ factories are believed to have a high potential to improve our understanding of $\mathrm{CP}$ violation in the universe [37]. The basic idea remains to overconstrain the UT, but this time using much more $B$ decays reconstructed with a better detector to reduce the errors. From an experimental perspective there are just two possibilities: either the UT remains closed and confirming the SM at higher accuracy. Or the UT begins to open and directly shows new physics. Other investigations at upgraded $B$ factories will search for very rare $B$ decays, effects from new Higgs particles, new flavour violation such as lepton-flavour violation and the existence of right-handed currents. These examples are very difficult to access at other currently running high-energy physics experiments.

\subsection{Belle II at SuperKEKB: Challenges and opportunities}

\subsubsection{The general goals of SuperKEKB}

The accelerator SuperKEKB and its detector system Belle II are an upgrade of the former KEKB Bfactory in Tsukuba, Japan. Like its predecessor, SuperKEKB is an asymmetric ring-accelerator that will collide electrons at $7 \mathrm{GeV}$ and positrons at $4 \mathrm{GeV}$ in order to produce boosted $Y(4 s)$ mesons that predominantly decay into pairs of $B$ mesons. The luminosity upgrade program of the accelerator aims at a 40-fold increase for the rate of electron positron collisions to acquire much more $B$ meson decays in the first ten years of operation than before. At the same time, the upgrade of the detector system aims at a significant improvement of the resolution for observation of $B$ decays. The upgrade has the potential to significantly lower the experimental uncertainties of the unitarity triangle within the first six years of operation [37, 11].

A schematic drawing of the SuperKEKB accelerator is shown in Fig.2.3. The accelerator will consist of two separate storage rings for bunches of electrons at $7 \mathrm{GeV}$ and positrons at $4 \mathrm{GeV}$ circulating nearly at the speed of light with a period of $10 \mu$ s per revolution. At nominal operation of SuperKEKB, 


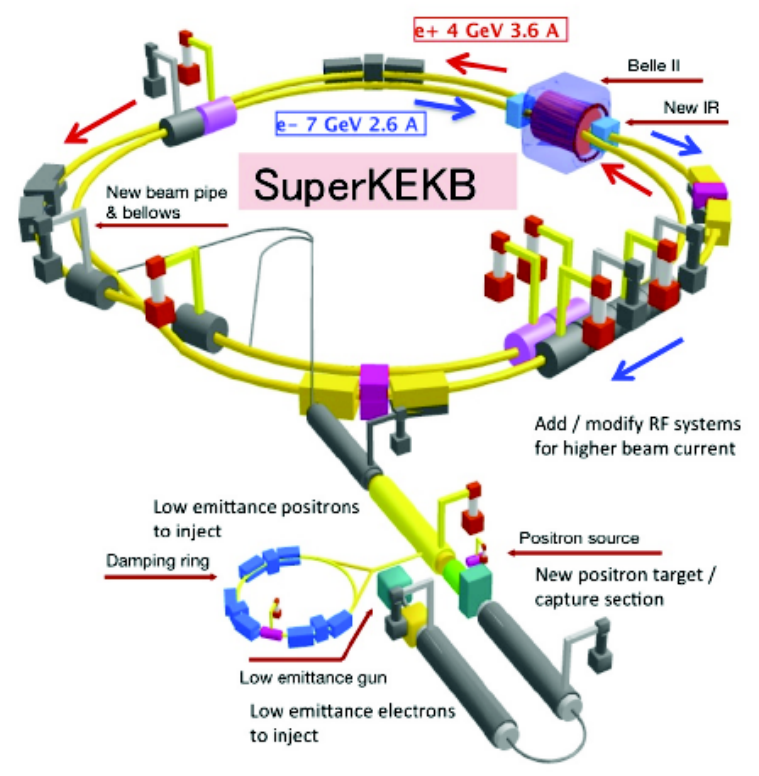

Figure 2.3.: Illustration of the SuperKEKB accelerator at Tsukuba in Japan. Areas with major changes with respect to KEKB are marked.

there will be around 2500 bunches per ring and each electron (positron) bunch will roughly contain $6.5 \times 10^{10}\left(9 \times 10^{10}\right)$ particles. The bunches will be injected into the storage rings from a new low emittance electron/positron gun at a continuous rate of $10 \mathrm{~Hz}$. The SuperKEKB accelerator has a single interaction point where electron and positron bunches are collided every $4 \mathrm{~ns}$. To enhance the possibility for electron-positron collisions, the cross section of the bunches at the interaction point will be strongly squeezed by quadrupole magnets to tens of nanometers. The crossing angle between of electrons and positrons will be $82 \mathrm{mrad}$ to achieve a better geometrical overlap between the bunches at the collision point. The upgrade scheme for the accelerator just outlined is called nano beam option and is fully described in reference [11]. From a physics perspective, the upgrade results in an increased rate of collisions or events where electrons and positrons annihilate at the interaction point to produce an $Y(4 s)$ meson containing a bottom $(b)$ and anti-bottom $(\bar{b})$ quark. The rate of such signal events increases from $0.5 \mathrm{~Hz}$ at KEKB to about $100 \mathrm{~Hz}$ at SuperKEKB. However, a rate of $100 \mathrm{~Hz}$ implies that signal events are still rather rare, while it does not imply that nothing is happening in other collisions. Physics interactions from these collisions are an important source of background that will complicate the observation of signal events.

The Belle II detector [11] is a hermetic detector system which allows the reconstruction of particle tracks after a collision and performs the measurement of energy, momentum and charge of the produced particles. A dedicated trigger system is used to steer the readout of the particle detectors after interesting collisions. A schematic illustration for such an interesting collision, the so called golden channel for $\mathrm{CP}$ violation, is shown in Fig.2.4. In the collision, a very short lived $Y(4 s)$ meson with a mean lifetime of $10^{-20} \mathrm{~s}$ is produced at the interaction point inside the beam pipe. Relative to the Belle II detector, the $Y(4 s)$ has a speed of $\sim 0.3 \times c$ in the flight direction of the more energetic electrons. Due to its short life time, the $Y(4 s)$ decays at the interaction point into a pair of neutral $B$ mesons which continue to fly along the beam line at high speed. However the $B$ mesons have a much longer mean lifetime $\sim 1 \mathrm{ps}$ 


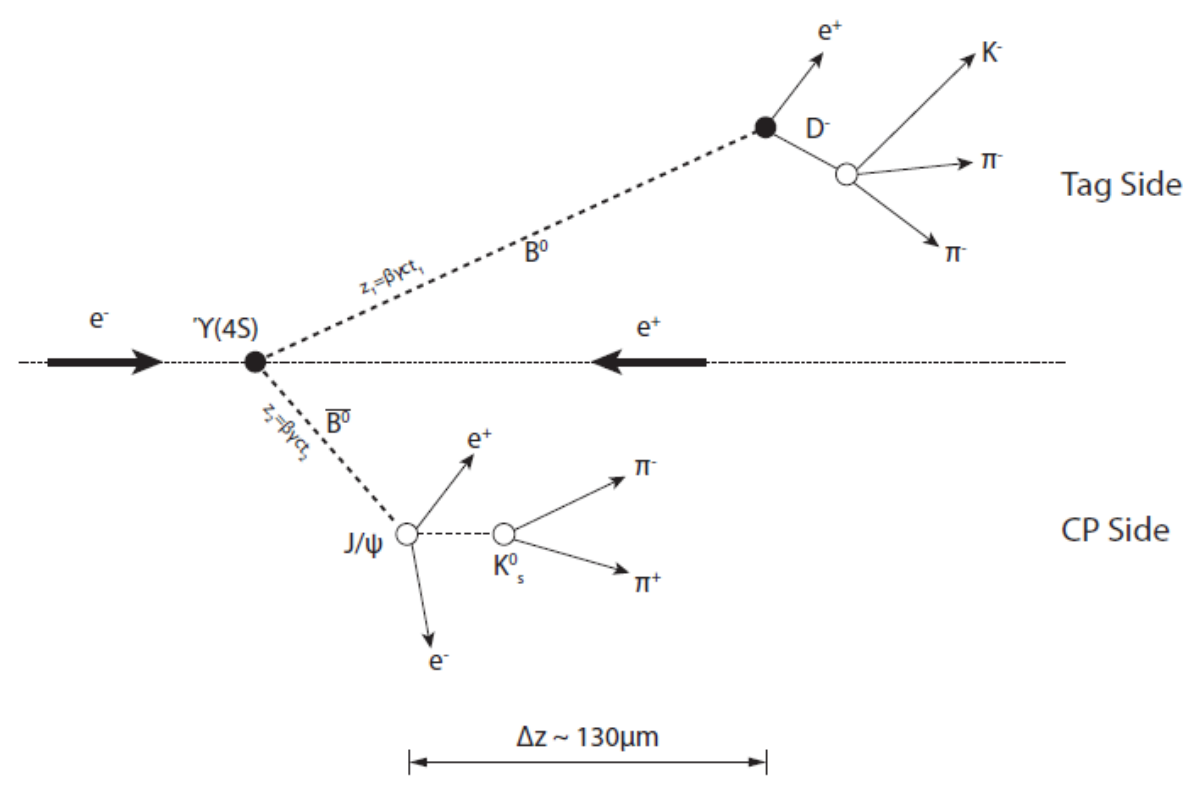

Figure 2.4.: Exemplary decay mode of neutral $B$ mesons to be studied with the Belle II detector. A coherent pair of neutral $B$ mesons is created at the interaction point in the beam pipe. The flavour of the $B$ mesons and the distance $\Delta z$ between $B$ decays must be reconstructed from stable decay products [38].

and decay at different positions forming two displaced decay vertices. The vertex displacement $\Delta z$ along the beam line observed from the detector is equivalent to a time difference $\Delta t$ between $B$ decays for a hypothetical observer sitting on the $Y(4 s)$ meson. The time difference $\Delta t$ plays an important role for precision measurements of time dependent $\mathrm{CP}$ violation in mixing and must be measured with high precision. The experimental trick of using asymmetric beam energies at $B$ factories displaces the decay vertexes and allows to separate the decay products along the beam line and to measure the time difference between decays.

\subsubsection{Vertexing challenges at Belle II}

The task of the pixel vertex detector (PXD) is to precisely detect the $B$ decay vertex from their longlived decay products. The goal of this section is to outline the specific challenges for vertexing at the interaction point of SuperKEKB in a way that is independent from a specific detector design.

A schematic view of the two layer PXD in the $r-z$ cross section along the beam line is shown in Fig. 2.5. From a conceptual point of view, the PXD readout delivers true $2 \mathrm{D}$ position coordinates relative to the surface of the thin silicon sensors for all charged particles emerging from a triggered collision. The silicon sensors are arranged in two cylindrical layers around the interaction point. The first task for vertexing is to collect all hits from the same particle at both layers and to compute a three dimensional model of the particle's flight path or track in the detector volume. The second task is the extrapolation of the track towards the interaction point. The track extrapolation allows a determination of the geometrical origin of the particle along the $z$ axis. However, the precision requirements for separation of decay vertices are very high as the vertex displacement $\Delta z$ at SuperKEKB will be as small as $\sim 80 \mu \mathrm{m}$ due to the reduced beam asymmetry compared to KEKB. Moreover, the problem is complicated by the fact that the upgrade of SuperKEKB strongly increased the rate and density of charged particles entering 


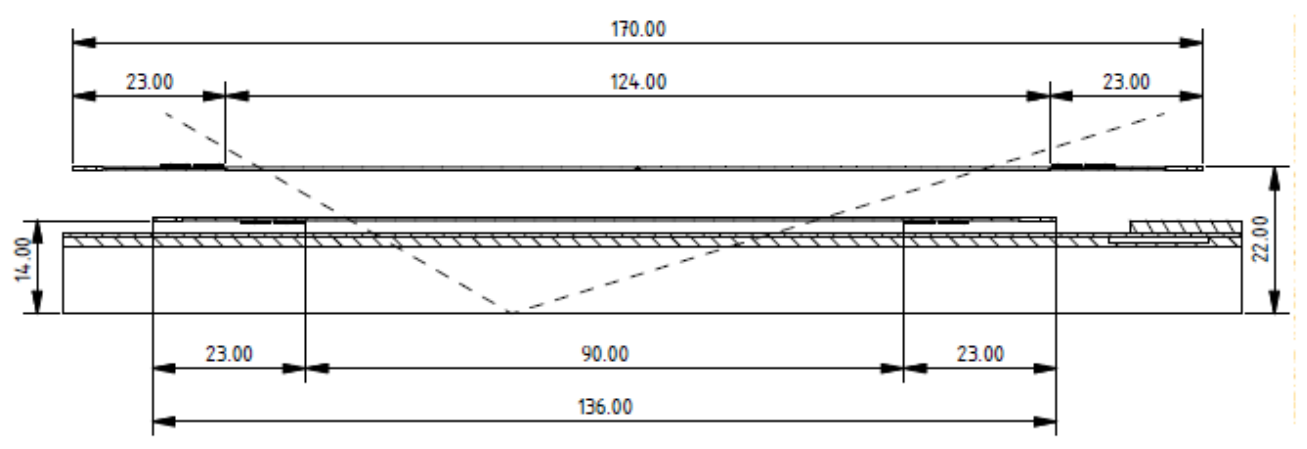

Figure 2.5.: Belle II PXD in $r-z$ plane: The view shows the interaction point, beam pipe wall and the two PXD layers at radii of $14 \mathrm{~mm}$ and $22 \mathrm{~mm}$. The PXD covers the full polar acceptance from 17 to 150 degrees around the interaction point.

the vertex detector both form $B$ decays and background sources. For this reason, the vertex detector events will contain a large number of hits per layer which complicates the identification of tracks from $B$ decays. In the following, an attempt is made to describe and quantify three particular challenges - vertex resolution, vertex acceptance and high rate capability - of vertexing at Belle II that drive the design of the pixel detector:

\section{Vertex resolution}

For simplicity, we restrict ourselves to a semi quantitative model for the single track $z$ resolution in the $r-z$ plane. The model determines the root mean square error $\sigma_{z}$ for the extrapolation of a single track to the beam axis from position measurements on two pixel layers as a quadratic sum of two terms [39]

$$
\sigma_{z}=\sqrt{\frac{r_{1}^{2}+r_{2}^{2}}{\left(r_{2}-r_{1}\right)^{2}}} \sigma_{s p} \oplus \frac{r_{1} \cdot 0.0136 \mathrm{GeV}}{p[\mathrm{GeV}] \cdot \sin ^{\frac{3}{2}} \theta} \sqrt{\frac{X}{X_{0}}}
$$

The first term is purely geometric and depends on the two layer radii $r_{1}$ and $r_{2}$ as well as on the intrinsic spatial resolution of the PXD layers $\sigma_{s p}$. The source of the second term are many small Coulombic scatterings of the charged particle in the innermost PXD layer leading to an additional random smearing of the polar angle $\theta$ as a function of the layer thickness $X$ per radiation length $X_{0}$. At Belle II, the particle spectrum is dominated by charged tracks with low momentum $p \sim 0.1-1 \mathrm{GeV}$ and multiple scattering dangerously degrades the vertex resolution.

For Belle II, the resolution $\sigma_{z}$ should be in the order of $\sim 10 \mu \mathrm{m}$ to allow a separation between displaced decay vertices. For the nano beam design of the SuperKEKB accelerator, the outer circumference of the beryllium beam pipe is reduced to $10 \mathrm{~mm}$. This is good for vertex resolution and physics, because it allows to shrink the inner layer radius to $14 \mathrm{~mm}$ and the outer radius to $22 \mathrm{~mm}$. At the same time, it is necessary to limit the overall thickness per layer to a very low level $<0.2 \%$ of $X / X_{0}$ to further minimize the multiple scattering term. The first term in Eq.2.1 leads to the requirement of a spatial resolution $\sigma_{s p}$ in the range of $\sim 10 \mu \mathrm{m}$. 


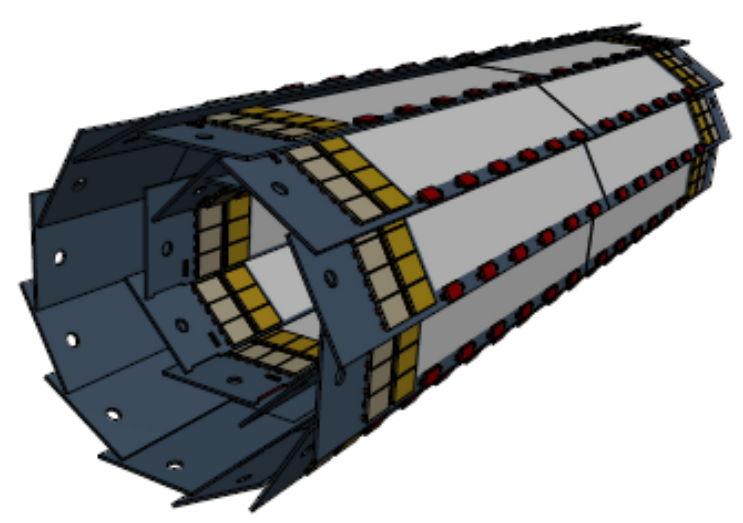

Figure 2.6.: Schematic view of the geometrical arrangement of the sensors for the PXD. The light grey surfaces are the sensitive pixel matrices, which are thinned to $75 \mu \mathrm{m}$ and cover the entire acceptance of the vertex detector. The full length of the outer modules is $174 \mathrm{~mm}$.

\section{Vertex acceptance}

The Belle II vertex detector aims to cover a wide range of polar angles $\theta$ from 17 degrees to 150 degrees relative to the flight direction of electrons. This range is called the polar acceptance and determines essentially the required sensitive length of the silicon sensors to $90 \mathrm{~mm}$ in the first layer and $124 \mathrm{~mm}$ in the second layer, see also Fig.2.5. As shown in Fig.2.6, eight silicon sensors with a sensitive width of $15 \mathrm{~mm}$ form the first layer, while the second layer consists of 12 sensors with identical width. The total active silicon area in the inner layer is roughly $110 \mathrm{~cm}^{2}$ and $220 \mathrm{~cm}^{2}$ in the inner and outer pixel layers. The high spatial resolution must be maintained uniformly over the whole area.

It should be emphasized that the areas outside of the polar acceptance do not contribute to the layer thickness for vertexing. Tracks from the interaction point hitting these parts miss the silicon sensors and are lost for physics measurements. These areas can be used for mechanical support structures, and for active cooling of fast readout chips with high power dissipation.

\section{High rate capability}

A pixel vertex detector records not only the hits from a triggered bunch collision but all hits in a fixed length time interval after the trigger as well. This so called integration time must be short enough to limit the number of hits per layer to a manageable level and to allow a unique identification for hits from $B$ decays. At SuperKEKB the situation is more challenging for two reasons. Firstly, the luminosity upgrade implies an increase in the number of tracks per bunch crossing by a factor of $10-20$. Secondly, the reduction of the layer radii improves the vertex resolution but at the same time increases the track density even more.

\subsubsection{The DEPFET pixel detector}

The main challenge for the design of the pixel vertex detector at SuperKEKB is to find a sound compromise between time resolution, layer thickness and pixel sizes, balancing the need for acceptable hit density and high vertex resolution. Obviously, simulation studies are needed to quantitatively compare different design scenarios. Especially the following two factors have played a crucial role in the final 
proposal: first, detector operation capabilities under increased level of particle background, and second, high position sensitivity to low momentum particles. From this perspective, only a vertex detector consisting of low material budget sensors, providing true $3 \mathrm{D}$ position measurements 1 and located as close as possible to the interaction point can fulfill such conditions. Particle detection with pixel matrices consisting of fully Depleted Field Effect Transistors (DEPFET) has been demonstrated to feature high signal to noise ratios $\sim 20-40$ for particle detection on thinned silicon sensors and a very low power dissipation in the acceptance region to avoid active cooling. A detailed description of the joint efforts of the DEPFET Collaboration towards the design of the DEPFET pixel detector for Belle II can be found in the Belle II Technical Design Report [11] and the references therein.

The PXD is the innermost part of the Belle II detector, mounted directly on the beam pipe around the interaction point. It has two layers with 8 million pixels in total. The integration time to readout an 8 bit digital signal from all pixels is $20 \mu$ s or two revolution cycles of SuperKEKB. The total raw data rate is $\sim 30 \mathrm{~Gb} / \mathrm{s}$. A fast detection of hit pixels allows to reduce the raw data rate by roughly a factor of $100 \mathrm{on}$ the level of pixel modules. A further reduction by a factor of 10 is required by the data acquisition system of the PXD where hits from all pixel modules are merged. The data reduction is achieved in real time by discarding hits far away from tracks observed in the outer tracking detectors of Belle II. The pixel pitch of modules varies with the $z$ position along the beam pipe. Small pixel pitches $50 \times 50 \mu \mathrm{m}^{2}$ are chosen in the inner part of modules close to the interaction point. In the forward-backward part of modules, the pixel pitches are enlarged to $50 \times 85 \mu \mathrm{m}^{2}$. This design takes into account the dependence of the intrinsic spatial resolution on the track incidence angle and allows to maintain an intrinsic spatial resolution of $\sim 10 \mu \mathrm{m}$ throughout the entire acceptance. The single track vertex resolution is $15-30 \mu \mathrm{m}$ depending on momentum and incident angle. For low energy momentum tracks, the single track resolution will be a factor of two better than in the previous detector.

A schematic drawing of the pixel vertex detector is shown in Fig.2.6. The PXD consists of two cylindrical layers around the beam pipe. The first layer is divided into eight ladders while the outer layer has 12 ladders. Each ladder consists of two pixel modules glued together by a ceramic joint in the middle. The inner part of the module is the sensitive pixel matrix thinned to $75 \mu \mathrm{m}$ while the periphery is a thicker silicon support frame and provides mechanical stability for the module. The support frame is populated by readout chips: six Switcher-B chips are placed on the balcony next to the long side of the matrix and four pairs of DCD-B and DHP chips are placed at the end of the pixel module outside of the acceptance where active cooling is possible. The pixel modules are powered independently and are readout in parallel to reduce the time needed to acquire data from all pixels in response to a trigger. Apart from the data acquisition system needed to merge hits from different modules, one can consider a pixel module as the basic functional unit of the PXD covering a small part of the Belle II acceptance.

\subsection{Scope and design of research: Three steps towards a valid detector simulation}

Simulation studies of the pixel vertex detector at SuperKEKB are an important method to prove the feasibility of the vertex detector design and demonstrate superior resolution beyond the previous detector.

\footnotetext{
${ }^{1}$ A pixel detector detects two hit coordinates relative to a planar silicon wafer. Hits are bound to a thin sensor plane and the third coordinate can be computed from the position and orientation of the sensor plane in space.
} 
The contribution of this thesis will be presented in three steps. First, we present a simulation algorithm which allows to study the response of a DEPFET pixel module hit by a charged particle. The validation of the simulation is a key topic as the design of the vertex detector and the prediction of an improved $\Delta z$ vertex resolution for Belle II depend on it. Secondly, we present new methods to analyze data from the EUDET beam telescope in the ILCSoft framework needed for the experimental validation from real data. The performance of the new methods is studied on pseudo experiments and on real data. The third step is the final validation using real data from the DEPFET prototype module operated inside the EUDET beam telescope.

\subsubsection{First step: Simulation of a vertex detector module}

A key part for the optimization of the vertex resolution is a realistic algorithm to simulate the response of a DEPFET pixel matrix to the hit of a charged particle. Such a simulation encodes the physical relationships between pixel size, sensor thickness and readout noise on the one hand and the intrinsic spatial resolution on the other hand. The focus of the study presented here is to understand the influence of the physical factors relevant for particle detection on the intrinsic spatial resolution on a quantitative level. The response simulation needs a detailed simulation of how signal charge is created inside the detector, shared between the pixel cells by drift and diffusion and finally converted to a digital number for the detected charge per pixel. The integration time can be implemented by overlaying the energy losses from all tracks crossing the sensor in a time interval of $20 \mu$ s starting with the trigger signal.

\subsubsection{Second step: Data analysis with EUDET beam telescope}

The EUDET beam telescope is a tool to study the response of real silicon pixel detectors $2^{2}$ to real charged particles. The EUDET beam telescope provides the infrastructure where the response from a pixel detector can be directly related with its source, namely the path of a charged particle of given mass, charge and momentum traversing the sensitive silicon volume. In order to achieve this, several pieces are needed: an intense particle beam, a fast trigger for charged particles, precise pixel detectors for charged particles, and a data acquisition system. The EUDET telescope offers all these parts as a service for research and development for precise tracking detectors in high energy physics [17].

A photograph of the EUDET beam telescope installed in the test beam area at DESY is shown as Fig.2.7. The telescope consists of two arms equipped with three high resolution pixel detectors each, which provide reference hits for charged particles used for tracking. The telescope mechanics provides a track system for a precise positioning of the reference pixel detectors along the beam line. In the space between the arms, a DEPFET pixel detector is installed as device under test (DUT) in the particle beam line. The DUT box is mounted on a motor stage which allows precise positioning of the DEPFET pixel matrix in the electron beam. In addition, the DUT box can be rotated with a precision of $10^{-3}$ radians to vary the inclination of tracks into the sensitive silicon for so called tilt scans. As a triggering device, two pairs of crossed finger scintillators are installed in front and behind the whole assembly. The scintillators are connected to a Trigger Logic Unit (TLU) which delivers a fast trigger signal to all detectors on the coincidence of all scintillators to steer the detector readout. All detector data for a trigger is sent to a common data acquisition system EUDAQ running on a PC where the data is merged to an event and

\footnotetext{
${ }^{2}$ More generally, position sensitive particle detectors
} 


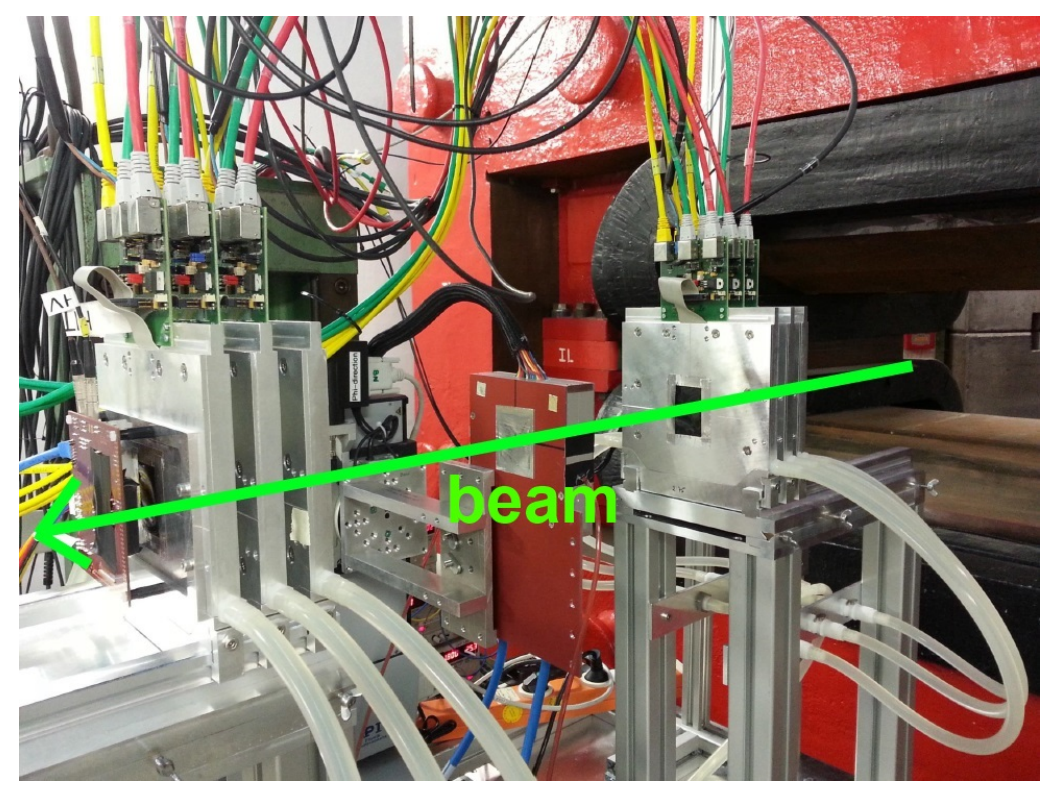

Figure 2.7.: Photograph of the EUDET beam telescope: Experimental setup installed at DESY beam line. A DEPFET prototype module is installed in the center. The pixel matrix is positioned in the electron beam.

written to file. EUDAQ is also used for the control of the telescope, starts and stops the data acquisition and provides online monitoring.

The usefulness of the EUDET telescope results from the very high intrinsic spatial resolution and low radiation length of the six telescope reference layers as well as the possibility to synchronize the reference telescope layers and the DUT. The six telescope layers are equipped with Mimosa26 [40] silicon pixel sensors with $18.4 \mu \mathrm{m}$ pixel pitch, and a silicon thickness of $50 \mu \mathrm{m}$. The Mimosa26 detectors cover an active area of $1 \times 2 \mathrm{~cm}^{2}$ and have an integration time of $115 \mu \mathrm{s}$. The maximum trigger rate of the telescope is $\sim 1 \mathrm{kHz}$. The spatial resolution of the Mimosa26 sensors depends on their operation mode 4 , but values between $3.5 \mu \mathrm{m}$ and $4.5 \mu \mathrm{m}$ are reported in the literature [41].

The combination of spatial resolution and low material offers a precise estimation of the hit position and the incidence angle of particles at the DUT. These local track parameters should be estimated with the highest possible precision. However, the precision depends on a number of factors like the momentum of beam particles, the precise information about detector positions in space and the distance between detectors. Last but not least, the optimal estimation of track parameters requires to explicitly take into account the Coulombic scattering of the particle when traversing material in the telescope. Relevant materials are the detectors and even the air between detectors. In chapter 4, we present our approach to proceed from pixel hits on the telescope to the estimation of track parameters at the DUT. The quality of data analysis methods is studied in chapter 4 on real data and in appendix $\mathrm{A}$ on pseudo experiments.

\footnotetext{
${ }^{3}$ The Mimosa26 sensors are packaged in boxes with light shielding windows at the front and back side. These windows consist of $25 \mu \mathrm{m}$ carbon and must be added to the overall radiation length per telescope layer. The resulting radiation length per layer is $X / X_{0}=0.07 \%$.

${ }^{4}$ The threshold for particle detection can be chosen by the user. Optimal values are $5-6 \times$ readout noise.
} 


\subsubsection{Third step: Validation of simulation results}

After having presented the simulation algorithm for DEPFET pixel modules and the data reconstruction for charged particles in the EUDET beam telescope, the final part of this thesis covers the validation of the simulation using beam telescope data.

Previous results of DEPFET pixel detectors from high energy beam tests used $450 \mu \mathrm{m}$ thick sensors with very small $20 \times 20 \mu \mathrm{m}^{2}$ pixels. These test beams were focused on the functionality of small pixel designs relevant for the International Linear Collider and to demonstrate a spatial resolution around $\sim 1 \mu \mathrm{m}$ [42]. The test beams relevant for the validation of the DEPFET Digitizer for Belle II are based on the latest DEPFET sensor production on $50 \mu \mathrm{m}$ thin silicon with enlarged pixel sizes of $50 \times 50 \mu \mathrm{m}^{2}$ or $50 \times 75 \mu \mathrm{m}^{2}$ relevant for Belle II. Another important addition is the replacement of the outdated CURO chips with the DCD-B readout chip designed for Belle II [15].

The first part of the validation study is the calibration of the overall readout noise in the prototype detectors. The readout noise is estimated pixel by pixel from the fluctuations of the digitized drain currents. The proposed scheme to calibrate and convert digitized currents into an equivalent number of collected electrons employs the well known distribution for energy loss straggling in $50 \mu \mathrm{m}$ of depleted silicon. We find that the readout noise is equivalent to $\sim 120$ electrons in the internal gate. The energy loss straggling is found to be well reproduced from simulation with Geant4.

The validation study focuses on the study of the spatial resolution of the pixel module as this is ultimately the most relevant quantity for a vertex detector. The spatial resolution is studied for ultra relativistic hadrons, mostly protons and pions, with a momentum of $120 \mathrm{GeV}$ at the $\mathrm{H} 6$ area at CERN and $1-6 \mathrm{GeV}$ electrons for test beams at DESY. The spatial resolution was measured as a function of the beam inclination for angles up to 50 degrees. The test modules are operated without the DHP chip [43] and provide a unique opportunity to study the dependence of the spatial resolution on the zero suppression threshold. The best spatial resolution is achieved for a rather low threshold equal to $5 \times$ the equivalent noise charge. For lower thresholds, the resolution is compromised by readout noise while for higher thresholds the detected charge sharing is too small.

The high position resolution of the EUDET telescope is exploited in a further study of the in-pixel signal charge collection with tracks hitting the sensor at perpendicular incidence. This situation offers the possibility to search for in-pixel areas with incomplete charge collection by mapping the average cluster charge as a function of the in-pixel hit position. Similarly, we can map the average cluster size to identify in-pixel areas with large charge sharing to neighbouring pixels. In the first case, the average cluster charge drops by $15 \%$ in the corners of pixel cells. The detector simulation reproduces this charge loss and hits at the zero suppression threshold as the most probable explanation. In the second case, the DEPFET response simulation reproduces the observed charge sharing well. Finally, we present a study for the hit detection efficiency of the tested DEPFET modules. The hit detection efficiency is found to be $>99.5 \%$ for all working pixels. 


\section{The DEPFET detector simulation: Algorithm and its implementation}

This chapter presents a computer simulation algorithm for the position resolved detection of a charged particle hitting a DEPFET pixel detector. The computer simulation allows to predict the intrinsic spatial resolution of the novel design DEPFET pixel detectors for the Belle II vertex detector. As discussed before in section 2.2.2, a high intrinsic spatial resolution of $\sim 10 \mu \mathrm{m}$ for very thin pixel detectors is the key requirement for an improved $\Delta z$ vertex resolution from low momentum charged tracks at SuperKEKB.

Starting from a precise statement of the simulation problem in section 3.1, we continue to describe the software framework used to organize the simulation modules and manage the data flow between modules (section 3.2. In section 3.3, the simulation algorithm is described in three steps, going from energy depositions in the silicon sensor towards signal electrons stored in the internal gate of DEPFET pixels and finally to digitized charge measurements, or digits, delivered from the readout chips. In the last section of this chapter, we implement the algorithm to simulate a test beam experiment to estimate the intrinsic spatial resolution of DEPFET pixel detectors for Belle II.

\subsection{Introduction: Overview of simulation and its challenges}

The DEPFET detector simulation provides a computer model for the processes inside the pixel module beginning with the entry of a charged particle into the silicon sensor and ending with the detection of pixel signals or digits on disk, see Fig. 3.1. The detector simulation proceeds from "left to right" modeling the detection of energy losses along the path of charged particle in the silicon sensor. On the other hand, the hit reconstruction proceeds from "right to left" estimating the particle hit position from the detected cluster of digits. The implementation of a test beam simulation allows to proceed from "left to right" and back from "right to left" in order to estimate the error of the reconstructed hit position as a function of the true energy losses in the sensor. In particular, the simulation allows to extract the distribution of position errors of the pixel detector and predict its intrinsic spatial resolution.

The simulation of particle detection ("from left to right") can be modeled as a three step process, that results in a cluster of digitized measurements of how many signal electrons were collected by pixels close to the particle track:

- The first step is the simulation of the spatial distribution of energy losses along the particle's path in the silicon sensor. The energy losses are locally converted to a number of mobile electron hole pairs in the depleted silicon sensor. On the average, one electron hole pair is created per $3.62 \mathrm{eV}$ energy loss in silicon at room temperature. The holes drift undetected towards the back plane and can be ignored. Only the electrons will be detected in DEPFET pixels.

- The second step covers the collection of electrons into the internal gates of nearby DEPFET pixels. 

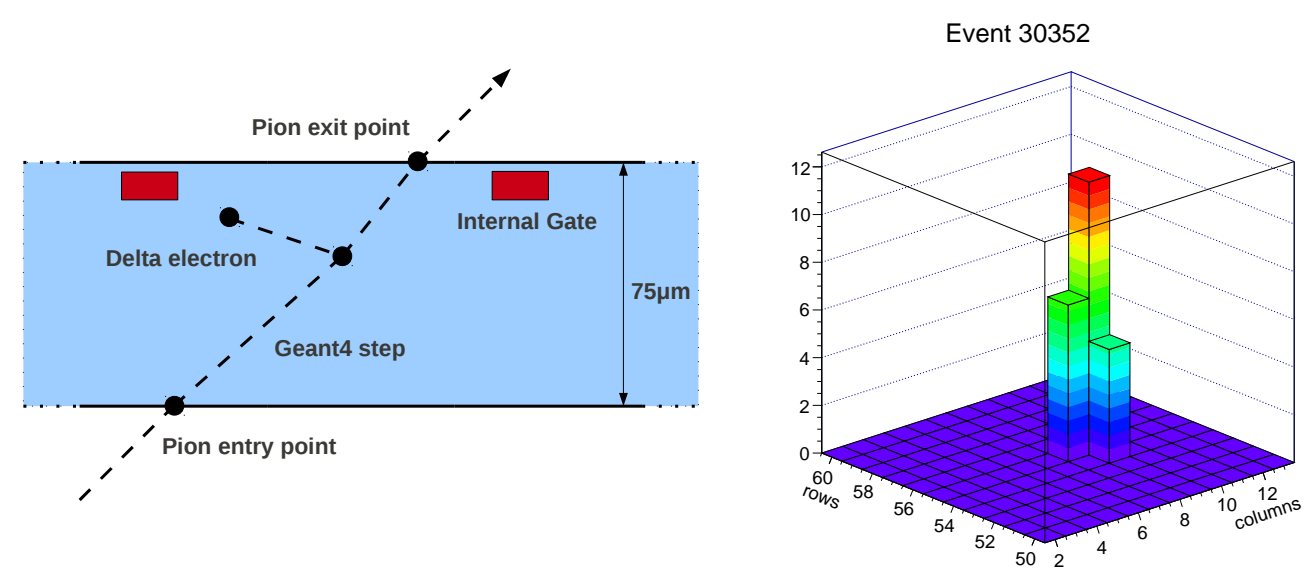

Figure 3.1.: Left: Schematic picture of the passage of a $120 \mathrm{GeV}$ pion in the silicon sensor simulated in Geant4 [44]. The position of the internal gate of two pixels close to the hit is indicated. Right: Schematic drawing of the detected cluster in response to the pion hit. The detected cluster contains three digits recorded from pixels around the hit position.

The charge collection is mostly based on fast drift in the sensor. However, in between two pixels, the drift fields are weak and charge transport is based on slow diffusion. At Belle II, a Lorentz shift of electrons in the $1.5 \mathrm{~T}$ magnetic field must be taken into account.

- The third step covers the pixel level amplification and digitization of the number of collected electrons at readout time. The signal height for a pixel is proportional to the stored number of electrons in the internal gate. In order to save computation time, the number of electrons is smeared by readout noise and a zero suppression threshold is applied.

While there are generic and high quality solutions available for the first step, the main innovation of this thesis is in the second and third step. The main challenges to be solved were the speed of the simulation and an accurate prediction of the intrinsic spatial resolution. Speed is an issue as the Belle II pixel detector has 8 million pixels which are readout at a rate of $50 \mathrm{kHz}$. Too much for a personal computer. To solve this issue, the philosophy of the approach presented here is to simulate the pixel data after digital calibration and zero suppression provided by the readout chips on the pixel modules. It means that no attempt is made to simulate the highly parallelized and fast operation of the analog and digital readout chips, but the simulation presumes their successful operation. This approach is justified by the high production yield which is required for readout chips on a final production pixel module for Belle II.

Regarding the second challenge: the intrinsic spatial resolution of pixel modules in the innermost layer is the key for the $\Delta z$ vertex resolution, but reference data to guide the sensor layout, in particular the thickness and the pixel size, were missing when the design of the Belle II pixel vertex detector was optimized in 2010-2011. Even today, no data from $75 \mu \mathrm{m}$ thin final design modules for Belle II are available for testing. To solve this issue, a realistic detector simulation was needed to accurately predict the response of final design modules to charged particles. The idea was to extend available simulations of the electric drift fields in fully depleted DEPFET sensors from $2 \mathrm{D}$ cuts into a $3 \mathrm{D}$ model for collection of electrons into the internal gates of DEPFET pixel cells. In particular, the model uses the 
physical equations for electron drift, diffusion and Lorentz shift in a magnetic field. Despite a number of simplifications, the developed charge collection model is demonstrated to correctly predict the intrinsic spatial resolution of $50 \mu \mathrm{m}$ thin prototypes available for test beams since end of 2011 .

The three steps described so far take us from a particle passage in silicon to readout digits ("from left to right"). The key for a position resolved particle detection ("from right to left") is now to estimate the position where the particle passed the sensor from the digits. This hit reconstruction consists of two steps: clustering and hitmaker. The task of clustering is to form a list, or cluster, containing all digits from the same particle passage. The next step, the hitmaker, processes clusters and computes a center-of-gravity of the position of pixels on the sensor weighted by their detected signal. The main task addressed here is to quantify the distance between the estimated and true position of particle passage on the silicon sensor. The intrinsic spatial resolution quantifies the width of the distribution of these distances, or hit measurement errors, in a way usable for tracking and vertexing [45].

The estimation of the intrinsic spatial resolution requires to combine all steps discussed so far in a simulated test beam experiment: Particles are repeatedly shot at a pixel module under identical conditions. The simulated particle passage is digitized and clustered. Finally, the intrinsic spatial resolution is taken as the root mean square of the histogram of measurement errors. The simulated test beam experiment allows to study the impact of different physical effects on the spatial resolution. In particular, the incidence angle of the particle relative to the sensor plane and different scenarios for the electronic noise and zero suppression threshold have a sizable impact on the intrinsic spatial resolution.

\subsection{The software framework: Tools and methods for data simulation and reconstruction}

\subsubsection{Key components of the ILCSoft framework}

The simulation of the particle passage through silicon sensors, the digitization of energy losses and the hit reconstruction are implemented in the ILCSoft framework developed for detector studies of the International Linear Collider [46, 47]. The structure of ILCSoft is shown in Fig. 3.2. The key components of the framework used in this study are Mokka, Marlin, LCIO and GEAR:

- Mokka is a stand alone program based on the Geant4 toolkit [44] used for the simulation of the passage of particles through a setup of silicon absorbers. Particles are shot one after another and a simulated event contains the energy losses in all silicon absorbers traversed by a particle. Mokka reads the telescope geometry from a MySQL database that defines the geometrical positions of the silicon sensors. Mokka uses the LCIO [48] data persistency framework to write the output data event by event to an LCIO output file. An event is represented by an LCIO::LCEvent object and stores the energy losses in a collection of type LCIO::SimTrackerHit. A SimTrackerHit represents a Geant4 step inside a silicon sensor.

- Marlin is a modular application framework used to organize the reconstruction into a flow of modular processing units called Processors. Processors are used to implement independent computations like digitization or clustering in $\mathrm{C}++$ classes that process data stored in an LCIO file from simulation event by event. A Marlin Processor has well defined interfaces to read input data from and write output data to an LCEvent. Information about the detector geometry is available 


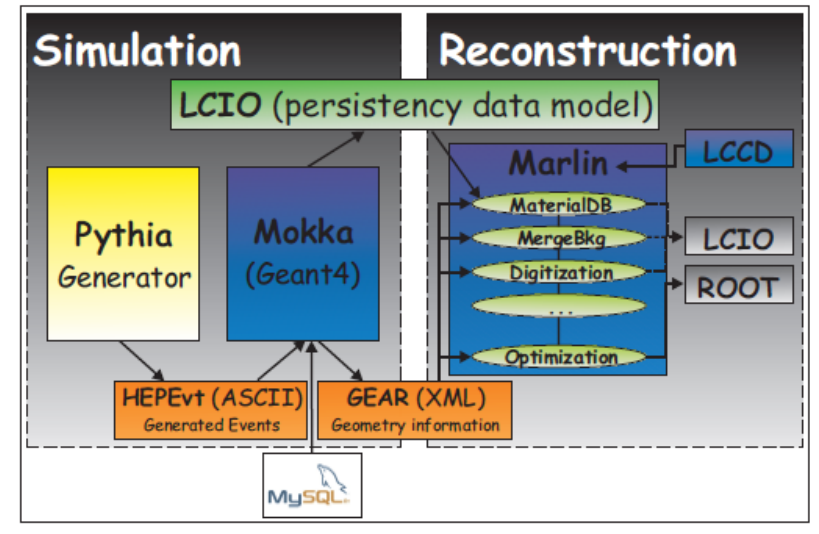

Figure 3.2.: Scheme of ILC software framework for data simulation and reconstruction [14].

from a Gear file for all Processors. Additional user variables needed in Processors can be supplied in steering files.

- GEAR offers an XML based format for the description of the tracking telescope as an array of detector modules called layers. The GEAR file contains all needed information for the digitization of energy losses or the reconstruction of hits. For example, it stores and organizes specifications of the length, width and height of the silicon sensor as well as the number of pixel columns (rows) and the radiation length.

- LCIO offers an object oriented and persistent storage for event data at all steps of the reconstruction flow. Data objects are stored event by event. Data objects like LCIO::SimTrackerHits, LCIO::TrackerData and LCIO::Hits are used to organize data for Geant4 steps, digits, hits and tracks in an event by event fashion.

Necessary adjustments and specifications to these tools are described within the steps of the simulation approach or the hit reconstruction.

\subsubsection{The geometry model for a beam telescope}

The simulation of a test beam experiment requires a detailed description of the geometrical layout of silicon sensors. As already discussed in section 2.3.2, a beam telescope consists of an array of silicon sensors, and their readout electronics, positioned along a particle beam line. One of the challenges for writing a detector simulation and reconstruction for a beam telescope is to organize the geometry related data in an object oriented way. Digitization requires to relate the $3 \mathrm{D}$ position of energy losses to the positions of pixels cells on the sensor for the simulation of charge collection. Hit reconstruction needs to know the position of pixel cells on the sensor plane to estimate the hit position of a particle from digits. Both tasks need to be solved for different sensor layouts and arbitrary rotations of the silicon sensor with respect to the particle beam. The philosophy followed here is to perform all these computations in local coordinate systems attached to the silicon sensors. Local coordinate systems allow to decouple the description of the sensor layout from its placement in the laboratory. This approach is possible since the detection of energy losses and all related quantities like digits, clusters and hits are inherently local 


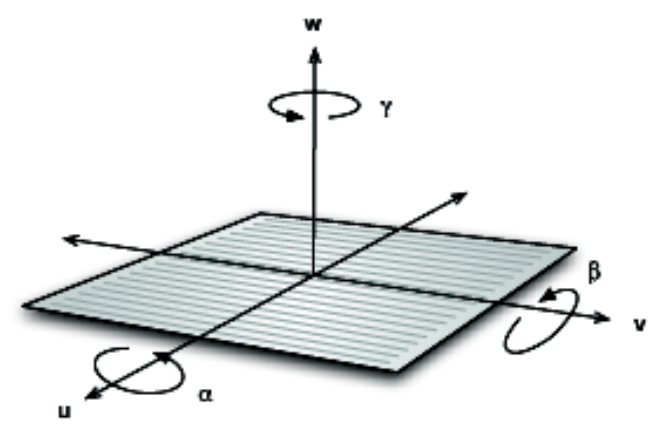

Figure 3.3.: Definition of the local coordinate system for a silicon sensor. For a pixel detector, the $u$ axis points along the readout columns while the $v$ axis points along the readout rows. The $w$ axis is the sensor normal and forms a right handed $u, v, w$ coordinate system.

and involve only interactions of a particle with a single silicon sensor in the beam telescope. The local coordinate approach will be used both for the digitization of energy losses and the reconstruction of hits on individual silicon sensors.

In the following, we introduce the mathematical description of the local coordinate system approach and the $\mathrm{C}++$ class hierarchy needed to organize geometry data.

\section{Local coordinate systems}

A local coordinate system $(u, v, w)$ is attached to each silicon sensor. The origin of the local coordinate system is the center of the sensitive volume at half thickness, half length and half width as shown in Fig. 3.3. The $u$ and $v$ axis are aligned with the $2 \mathrm{D}$ pixel matrix and point along the direction of increasing column $(i)$ and row $(j)$ pixel indices. The $w$ axis is perpendicular to the silicon sensor surface and completes a right handed Cartesian coordinate system. The $w=0$ plane is called the measurement plane of the layer. All particle intersections with the silicon sensor will be computed relative to the measurement plane.

The transformations between local and global coordinates are managed by the class ReferenceFrame. The user interface prescribes methods to transform the coordinates of space points and direction vectors from global coordinates to local coordinates and vice versa. A space point with coordinates $\vec{r}=(x, y, z)$ may be transformed to layer coordinates $\vec{q}=(u, v, w)$ using the transformation law

$$
\vec{q}=R_{0}\left(\vec{r}-\vec{r}_{0}\right)
$$

where $R_{0}$ is a $3 \times 3$ rotation matrix and $\vec{r}_{0}=\left(x_{0}, y_{0}, z_{0}\right)$ defines the sensor origin in telescope coordinates. Essentially, the pair $\vec{r}_{0}$ and $R_{0}$ embeds a silicon sensor in a common telescope coordinate system. The telescope coordinate system $(x, y, z)$ is a right handed Cartesian coordinate system where the $z$ axis points along the particle beam, and the $y$ axis points upward.

In the typical case, silicon sensors in a beam telescope are placed in regular distances along the $z$ axis. Sensors are exactly perpendicular to the $z$ axis and get crossed by beam particles at near perpendicular incidence. We can count a total of eight discrete rotations $D$ to embed the local coordinate $u v w$ axes into the tracker volume 


$$
D=\left(\begin{array}{ccc}
d_{1} & d_{2} & 0 \\
d_{3} & d_{4} & 0 \\
0 & 0 & d_{5}
\end{array}\right)
$$

The discrete rotation has four parameters $d_{i}=( \pm 1,0)$. The fifth component $d_{5}$ is uniquely determined by the condition to form a proper $3 \times 3$ rotation matrix. The four rotation parameters are specified in the gear file. They account for the various possibilities to attach a detector module to the telescope support table.

Apart from the discrete rotation, sensors are allowed to have additional continuous rotations in the laboratory. A continuous rotation matrix may be written as a product $R_{C}=R_{3}\left(\gamma_{0}\right) R_{2}\left(\beta_{0}\right) R_{1}\left(\alpha_{0}\right)$ using the following three Euler rotations

$$
\begin{aligned}
& R_{1}(\alpha)=\left(\begin{array}{ccc}
1 & 0 & 0 \\
0 & \cos \alpha & \sin \alpha \\
0 & -\sin \alpha & \cos \alpha
\end{array}\right) \\
& R_{2}(\beta)=\left(\begin{array}{ccc}
\cos \beta & 0 & \sin \beta \\
0 & 1 & 0 \\
\sin \beta & 0 & \cos \beta
\end{array}\right) \\
& R_{3}(\gamma)=\left(\begin{array}{ccc}
\cos \gamma & \sin \gamma & 0 \\
-\sin \gamma & \cos \gamma & 0 \\
0 & 0 & 1
\end{array}\right)
\end{aligned}
$$

Initial values for layer rotation angles $\alpha_{0}, \beta_{0}$ and $\gamma_{0}$ are specified in the gear file. The overall rotation matrix of a sensor relative to telescope coordinates is a matrix product of the discrete and the continuous rotation matrix

$$
R_{0}=R_{C} D=R_{3}\left(\gamma_{0}\right) R_{2}\left(\beta_{0}\right) R_{1}\left(\alpha_{0}\right) D
$$

In total, the position and rotation of a sensor in the telescope is determined by six continuous geometry constants $\left(x_{0}, y_{0}, z_{0}, \alpha_{0}, \beta_{0}, \gamma_{0}\right)$ and four discrete rotation parameters $\left(d_{1}, d_{2}, d_{3}, d_{4}\right)$. The sensor can be shifted and rotated in the telescope by shifting the center coordinates $x_{0}, y_{0}, z_{0}$ or rotated (tilted) by changing the Euler angles $\alpha_{0}, \beta_{0}, \gamma_{0}$.

\section{Class hierarchy: Telescope and sensors}

In the case of a beam telescope experiment (test beam), the telescope geometry is a linear array of silicon pixel detector modules called telescope layers or planes. The telescope layers are naturally numbered along the beam line. The geometry data provided by the gear file is organized in an object oriented way with a two layer hierarchy. On the top level, the class TBDetector represents the entire telescope with all telescope layers. On the second level, the class Det represents a telescope layer and provides a common interface to retrieve layer specific geometry data. Together, the TBDetector and Det classes provide a common interface for all simulation ('digitization') and data reconstruction ('clustering', 'hitmaker, 'tracking','alignment') Marlin Processors used in this thesis. 
The TBDetector class provides an interface to read the geometry of a beam telescope from a GEAR. It initializes Det objects for all telescope layers found in the GEAR file. The Det class provides an abstract interface to the geometry data stored in the GEAR file for individual telescope layers. The layout of GEAR files used in this thesis is introduced in appendix C. A telescope layer has a unique DAQID which allows to relate a layer to its readout data in the LCIO file. The Det class provides an abstract interface to data reconstruction methods. The interface prescribes methods for the following common tasks:

- Transform between local coordinates $(u, v, w)$ and telescope coordinates $(x, y, z)$.

- Get column $i$ and row $j$ of the pixel struck by a track intersection at $(u, v, w=0)$.

- Get geometrical center $\left(u_{c}, v_{c}, w_{c}=0\right)$ of the pixel cell with column $i$ and row $j$.

- Get position $\vec{r}_{0}=\left(x_{0}, y_{0}, z_{0}\right)$ and rotation matrix $R_{0}$ of the silicon sensor.

- Get layer thickness $X(u, v, w=0)$ and radiation length $X_{0}(u, v, w=0)$.

- Get pixel pitch and intrinsic spatial resolution for pixel cell $(i, j)$.

- Have pixel cells $\left(i_{1}, j_{1}\right)$ and $\left(i_{2}, j_{2}\right)$ a common edge (corner) on the sensor?

This interface is sufficiently powerful to support all reconstruction algorithms used in the remainder of this thesis. The default implementation of the interface assumes a checkerboard arrangement of pixels on the sensor. The default implementation is already enough to support the current DEPFET and Mimosa26 detector layouts. New detector layouts can be integrated into the reconstruction framework by adding new specializations of the Det class.

\subsubsection{Event data model: energy losses, digits, clusters and hits}

The data model, which is used to describe the event data throughout the chain, is based on the LCIO persistency framework. It provides all necessary structures $(\mathrm{C}++$ objects) to store data produced in silicon simulation: energy losses (SimTrackerHits) $\rightarrow$ digits (TrackerData) $\rightarrow$ cluster (TrackerData) $\rightarrow$ hits (TrackerHits).

\section{Energy losses in silicon sensors}

The Mokka simulation creates a detailed map of all energy deposits of primary and secondary particles in the sensitive volumes passed by the primary particle as a collection of SimTrackerHits. The most important attributes of SimTrackerHit for digitization are:

- The unique DAQID of the sensitive volume for this step.

- The position $\vec{r}=(x, y, z)$ of the step center in units of millimeters.

- The time $t$ of the hit in units of nanoseconds.

- The 3-momentum $\vec{p}=\left(p_{x}, p_{y}, p_{z}\right)$ of the step in units of $\mathrm{GeV}$

- The path length $\Delta s$ in the sensitive material in units of millimeters 
- The energy loss $\Delta E$ along the step in units of GeV.

Conceptually, a SimTrackerHits represents a straight line step of the primary particle from an initial point

$$
\vec{r}_{\text {initial }}=\vec{r}-\Delta s \frac{\vec{p}}{2|\vec{p}|}
$$

to a final point at

$$
\vec{r}_{\text {final }}=\vec{r}+\Delta s \frac{\vec{p}}{2|\vec{p}|}
$$

inside the silicon sensor. For a charged particle, the energy loss $\Delta E$ is converted into electron hole pairs created uniformly along the step. The number of electron hole pairs created along the step depends on the energy loss and is given as

$$
N_{e-h}=\frac{\Delta E}{J}
$$

where $J=3.62 \mathrm{eV}$ is the average energy to create an electron hole pair in silicon at room temperature. For the simulation studies presented here, the interface ${ }^{1}$ between Mokka and the particle propagation in Geant 4 was implemented in a way that any step of the Geant 4 particle propagation is converted into an LCIO::SimTrackerHit. There is no merging of multiple Geant4 steps into bigger steps and all secondary particles created in the sensitive volume are kept. The precision of the energy losses written to the LCIO file equals the precision of the Geant 4 simulation itself. The first and last LCIO::SimTrackerHit of a primary particle in a silicon sensor are marked as 'entry' and 'exit' steps as shown in Fig. 3.1. These two special steps will be used later to compute the true hit position of the particle independent from the detected energy losses.

\section{Readout digits}

A readout digit is the signal of a pixel in a detector. More precisely, a readout digit is a measurement of total charge $Q_{i j}$ deposited in the pixel cell $(i, j)$ of the silicon sensor. Readout digits are generally zero suppressed, the detected energy deposit is above a zero suppression threshold. The attributes of a readout digit are:

- The column $i$ and row $j$ numbers of the pixel on the pixel matrix.

- The calibrated detector signal $Q$ in units of the electron charge $e$

The number of digits varies from event to event. The persistent storage of digits in an LCIO::LCEvent is handled using the LCIO::TrackerData class. This class is essentially a $\mathrm{C}++$ standard vector of floating point numbers named chargeValues and a 64bit field for meta data. The meta data field is used to store the DAQID of the pixel module owning the digit. Digits are serialized and stored into the vector chargeValues.

\footnotetext{
${ }^{1}$ The interface is implemented as a G4SensitveVolume class. The G4SensitveVolume is a hook for end users to access the transient information of individual Geant 4 steps during particle propagation in Geant 4 .
} 


\section{Clusters and hits}

A cluster is a group of neighboring digits on the same detector module originating from the same particle. The handling of clusters in an event is again done using the LCIO::TrackerData class. The LCIO::TrackerData object stores only the digits belonging to the same cluster.

A hit on the telescope layer is computed from the clusters. It contains a $2 \times 1$ matrix for the estimated hit coordinates in the $w=0$ plane.

$$
m=\left(\begin{array}{l}
u_{m} \\
v_{m}
\end{array}\right)
$$

In addition, the hit contains a $2 \times 2$ measurement covariance matrix to quantify the statistical error of the estimated hit coordinates. The hit measurement covariance matrix is written as

$$
V=\left(\begin{array}{cc}
\sigma_{u}^{2} & \sigma_{u v}^{2} \\
\sigma_{v u}^{2} & \sigma_{v}^{2}
\end{array}\right)
$$

where $\sigma_{u}$ and $\sigma_{v}$ are the intrinsic spatial resolution of the pixel module in the local $u$ and $v$ directions. The off-diagonal $\sigma_{u v}=\sigma_{v u}$ is the covariance between the measured hit coordinates $u_{m}$ and $v_{m}$. In many cases, the correlation between $u_{m}$ and $v_{m}$ is very close to zero and $V$ can be approximated by a diagonal matrix. It is important to realize that hits are bound to the measurement plane of the sensor. The $3 \mathrm{D}$ coordinates of the hit are $\vec{q}=(u, v, w=0)$ and local hit coordinates can be transformed into global hit coordinates using Eq. 3.1.

The storage of a hit is done via the LCIO::TrackerHit class. The implementation of the interface of the LCIO::TrackerHit class is changed in a way that the hit does not store the global coordinates $(x, y, z)$, but the triplet of local data $\left(u_{m}, v_{m}\right.$, DAQID) and the measurement covariance matrix $V$. The reason for this choice is that the local hit position $\left(u_{m}, v_{m}\right)$ is decoupled from the position and rotation of the sensor in the telescope. The Det object owning the hit has a member function to transform local hit coordinates into global hit coordinates on the fly. The framework is designed such that only TBDetector objects maintain information regarding the position and rotation of sensors in the beam telescope.

\subsection{The DEPFET simulation algorithm: A three step approach}

Based on the software framework and the mathematical models described above, we now present a detailed account of the simulation method. As already outlined, the basic idea is to work through three distinct steps which encapsulate different areas of detector physics needed:

- Step 1 deals with the physical interactions like ionization, multiple scattering and others between a charged particle and atoms in the silicon sensor. The main source for continuous energy losses along the particles path is ionization.

- In step 2, a physical model for the generation and collection of signal charge in DEPFET pixel matrix is presented. The approach is based on a realistic model for the electric and magnetic fields affecting drift and diffusion of signal charges.

- Step 3 describes the net effect of sensor readout, electronic noise and zero suppression, and converts the collected charge in pixel cells to readout digits. 
The simulation is presented on a technical level, focusing especially on the inclusion of a physics driven model for the collection of signal charge in step 2. The simulation method is well tested and used since 2011 for simulations of the pixel vertex detector at Belle II. Section 3.4 presents a scheme of how to predict the intrinsic spatial resolution from the simulation. Furthermore, the results of the simulation are compared to real data in chapter 5

\subsubsection{Step 1: From energy losses to signal charge deposition}

The general picture for energy losses of a relativistic charged particle in a depleted silicon sensor is the following: In absence of a magnetic field, the particle passes in a straight line through the silicon sensor and looses a small part of its kinetic energy in many collisions with shell electrons of silicon atoms. In silicon, the average energy loss of a relativistic charged particle is $\sim 300 \mathrm{eV}$ per micrometer path length [28]. The energy losses are uniform along the particles path and their detection allows to precisely follow the particle through the silicon sensor. However, to perform a realistic simulation, especially three physical effects must be taken into account: the energy loss per micrometer strongly fluctuates around its mean value, the particle is deflected due to multiple scattering and secondary electrons create ionization at a large distance from the primary particle. In more detail:

- Energy loss straggling: The straggling theory developed by Landau [49] allows to sample the ionization energy loss $\Delta E$ for a given track length $\Delta s$ of moderate silicon thickness $>10 \mu \mathrm{m}$. The default model for energy loss used in Geant4 [50] is based on a simplified model of a $\mathrm{Si}$ atom with two energy levels with binding energy $E_{1}$ and $E_{2}$. The atom-particle interaction can either be an excitation, with energy loss $E_{1}$ or $E_{2}$, or an ionization with a continuous energy loss distributed according to function $g(\Delta E) \propto 1 / \Delta E^{2}$.

- Delta electrons: Delta electrons are secondary particles created when the energy transferred to an atomic electron in a single interaction largely exceeds the ionization energy $I$ of silicon. In this case, the hit electron will fork out and deposit energy far away from the path of the primary particle. Delta electrons with an energy exceeding hundreds of keV are produced rarely but have a range of $>100 \mu \mathrm{m}$ in silicon. Long range Delta electrons ionize the silicon sensor far away from the primary track and drastically increase the cluster size.

- Multiple scattering: Coulombic interactions of the primary particle with Si nuclei lead to numerous random small angle scatterings of the primary particle in the silicon sensor. The default model for multiple scattering in Geant4 is reviewed in [51].

The physical interactions of the particle with matter are simulated with the GEANT4 [44] based Mokka program. The Geant 4 toolkit is the standard solution used to incorporate the above described physical processes correctly into the simulation of the passage of a charged particle through the silicon sensor. The generated particles are tracked through a three dimensional silicon volume representing the silicon sensor in the simulated test beam experiment. The particle passage is resolved in several so called G4Steps which represent a part of the particle track inside the sensitive silicon. Along a step of length $\Delta s$, the particle suffers a continuous energy loss $\Delta E$ due to ionization and excitation of $\mathrm{Si}$ atoms and random deviations of the flight direction due to multiple scattering. Furthermore, Geant 4 allows the creation of secondary particles in the silicon volume, for example Delta electrons created from large 
energy transfers from the primary particle to a silicon shell electron. The G4Steps provide an accurate and well validated three dimensional distribution of energy losses in the silicon sensor around the particle track and its secondary particles.

The precision of the Geant 4 simulation is controlled by a production threshold cut which has strong impact on the number of generated secondary Delta electrons. Generally speaking, if the energy of a Delta electron is below this threshold, no "soft" Delta electron is generated in the simulation but the transferred energy is counted as a continuous energy loss along the path of the primary particle. The choice of the threshold defines a compromise between computation time needed to simulate short ranged secondary particles and an accurate model of the spatial distribution of energy losses in silicon. As a rule of thumb, the threshold is chosen to simulate all Delta electrons which are able to traverse a mean distance of $1 \mu \mathrm{m}$ away from the primary particle.

\subsubsection{Step 2: Collection of signal charge in DEPFET pixels}

The result of step 1 is a map of energy losses or electron hole pairs in a silicon sensor. At this point, we need a physical model to describe how the DEPFET sensor detects electron hole pairs in the sensor volume and achieves a position resolved particle detection. For a DEPFET pixel matrix, the silicon sensor is fully depleted by a scheme called sidewards depletion [12]. The electron hole pairs in the sidewards depleted sensor are separated by the electrical field. Holes drift towards the back plane contact while electrons drift to the front surface and are collected in the internal gate of pixels. There, the electrons are stored for later amplification and detection. The task solved here is the main contribution of this work: the development of a fast simulation algorithm for the division of signal electrons between the internal gates of pixels close to the particle track, derived from two dimensional simulations of electrical fields in the DEPFET pixel sensor. After a brief overview of the functioning of the DEPFET pixel sensor, we move on to a detailed description of the new method.

\subsubsection{Signal detection with DEPFET pixel sensor}

The DEPleted Field Effect Transistor (DEPFET) represents a complex $3 \mathrm{D}$ device and a substantial simplification of detector physics is crucial for the digitization to perform in reasonable time. The DEPFET can be described as a MOS-type field effect transistor integrated on a sidewards depleted p-on$\mathrm{n}$ silicon sensor (Fig. 3.4). The device combines the advantages of large signals from charge collection in a fully depleted silicon sensor with low noise operation from in-pixel amplification. By means of sidewards depletion, a potential minimum for electrons is created right underneath the transistor channel (see right Fig. 3.4). The position of this so-called internal gate is shifted by a deep n-implantation to a depth of roughly $1 \mu \mathrm{m}$ below the sensor surface. When a particle creates electron hole pairs in the sensor volume, holes drift to the back contact and electrons are collected in the internal gate, where they accumulate. The signal charge leads to a change in the potential of the internal gate, resulting in a modulation of the channel current of the transistor at read-out time. After read-out, the signal charge is cleared out by a positive voltage pulse at the clear contact.

The DEPFET is operated dynamically by repeating a sequence of 'collect', 'read', and 'clear' states. In the collect state, the internal gate of a pixel collects and stores signal electrons. The duration of the collect state is called the integration time. In the 'read' state the gate voltage is pulled low and the DC drain current from the DEPFET transistor is sampled and digitized in the DCDB readout chip [15]. 

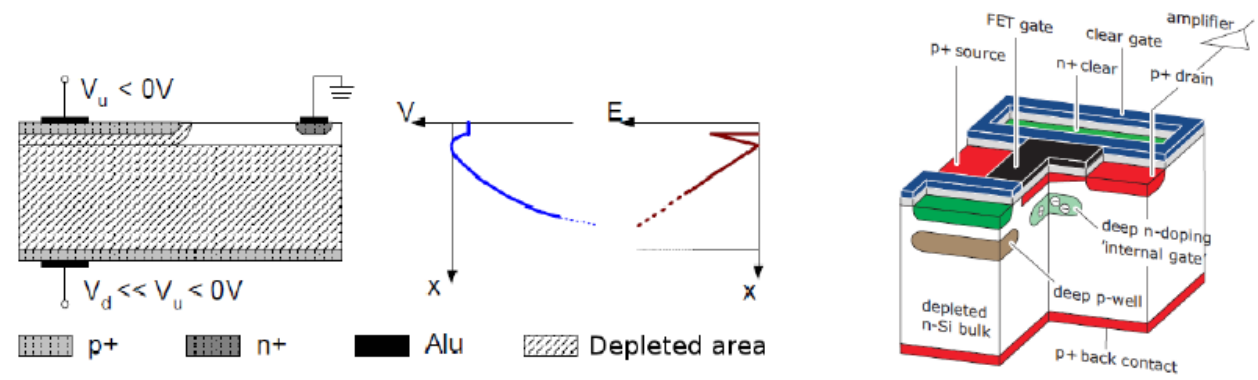

Figure 3.4.: Left and Middle: Cross section of a sidewards depleted $\mathrm{p}$ on $\mathrm{n}$ silicon detector and corresponding distribution of the electrical potential and electrical field. Right: $3 \mathrm{D}$ scheme of a DEPFET pixel cell [14].

The drain current $I_{d}$ is modulated by a capacitive coupling of the charge $Q$ in the internal gate to the transistor channel. The figure of merit is the internal amplification $g_{q}$ defined as $\Delta I_{d}=g_{q} \times Q$. In the 'clear' state a positive voltage pulse is applied to the clear contact and all electrons in the internal gate are transferred into the clear contact via a punch-through mechanism. The periodic repetition of the 'clear' state keeps the sensor bulk fully depleted and prohibits an overflow of the internal gate.

\subsubsection{Fast model for drift and diffusion in DEPFET pixels}

The model for the signal charge collection process in DEPFET pixel sensor is based on detailed semiconductor device simulations [52, 53]. The device simulation takes into account the detailed doping profiles of DEPFET sensors from the device manufacturing at the semiconductor laboratory of the Max Planck Society. Device simulations are available for the proposed prototype design for Belle II DEPFET sensors with a pixel size of either $50 \times 50 \mu \mathrm{m}^{2}$ or $50 \times 75 \mu \mathrm{m}^{2}$ on $75 \mu \mathrm{m}$ thick silicon substrate. The contact voltages are chosen to simulate a fully depleted DEPFET sensor in the 'collect' state. The device simulation provides maps of the electric potentials and electric fields for characteristic $2 \mathrm{D}$ cross sections through the $3 \mathrm{D}$ pixel cell along the local $u$ and $v$ directions. The sensor layout and the available cross sections are shown in Fig. 3.5.

The trajectories of signal electrons in fully depleted silicon can be described by the standard driftdiffusion equations in a magnetic field [54]. As can be seen from the equipotential lines in Fig. 3.6, the electric potential has large gradients (electric fields) along the local $w$ axis perpendicular to the sensor surface. The gradients along the lateral directions, the $u-v$ plane, are very small in most parts of the sensor volume. Between two pixels, a local potential minimum is achieved at a depth of $\sim 20 \mu \mathrm{m}$ below the front surface. In the following, we will call the $u-v$ plane at this depth the potential valley, see also Fig.3.6. Signal electrons arriving at the potential valley, are funneled by lateral fields into the internal gates. However, in between two pixels, the lateral fields are very small and charge transport is dominated by slow diffusion. This diffusion zone increases the charge sharing between pixels; signal electrons are distributed over more pixels. The numerical solution of the drift and diffusion equations for small groups of electrons is very time consuming and requires a complete $3 \mathrm{D}$ model of electric drift fields. In order to utilize the available $2 \mathrm{D}$ simulations and to save computation time, a simplified model for electron drift and diffusion was developed.

For this work, it will be assumed that the DEPFET sensor is operated in a way such that all signal 


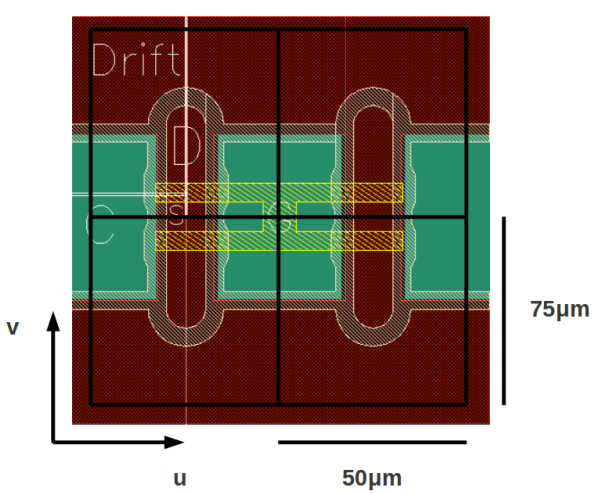

Figure 3.5.: DEPFET sensor layout for Belle II with four pixel cells on the sensor surface. The coloured regions are Clear ('C', green), Drift ('DRIFT', red), Source ('S', red), Drain ('D', red) implants and the Gate contact ('G', yellow). The first simulated cross section goes along the $u$ axis from the Gate into the Clear region. The second cross section goes along the $v$ direction from Source to Drift.

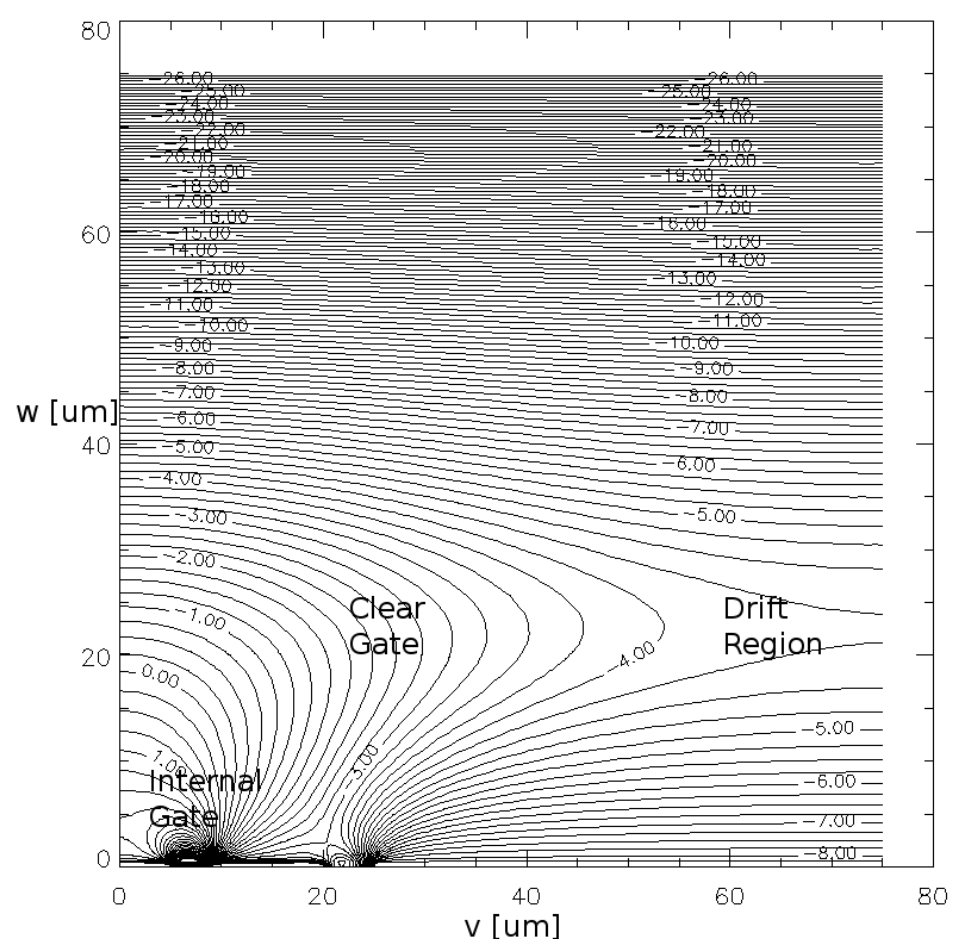

Figure 3.6.: $2 \mathrm{D}$ potential map for a Belle II sensor: The equipotential lines are shown for a cut along the $v$ axis from Source $\left(v_{S}=0 \mu \mathrm{m}\right)$ to Drift $\left(v_{D}=75 \mu \mathrm{m}\right)$. The internal gate is visible between Source and Drain roughly $1 \mu \mathrm{m}$ below the front surface. 
electrons created in the sensor bulk are collected within a charge collection time in the order of $\sim 10 \mathrm{~ns}$. The collection time is very short compared to the integration time of $20 \mu \mathrm{s}$. This situation implies that the time resolution of hits is limited by the integration time. In the following, we will assume that electrons have enough time to reach the internal gate before the next read-out cycle after the particle hit is performed. The task is effectively reduced to simulating how electrons are divided between the internal gates of pixels close to the particle track in the sensor. The key idea is to describe the collection of signal electrons from the point of creation until the arrival at an internal gate as a two step process with a 'vertical' and a 'lateral' part. In the 'vertical' part, all electrons are followed from their creation point into the potential valley assuming a purely parabolic electric potential along the $w$ axis. Lateral components of the drift field are neglected but lateral diffusion of electrons during the drift time and the Lorentz shift of electrons in a magnetic field perpendicular to the $w$ axis are taken into account. In the following 'lateral' part, electrons arriving in the potential valley are funneled into internal gates. The lateral funneling is rather simple in the case that electrons arrive in the potential valley close to an internal gate. In a well designed sensor, it is reasonable that those electrons quickly reach this internal gate by drift. However, the fate of electrons arriving in between two pixels where lateral drift fields are weak is not yet decided. These electrons can reach both neighbouring pixels by lateral diffusion. The idea is to subdivide the sensor area in drift and diffusion dominated areas as shown in Fig. 3.10 Electrons in a diffusion zone perform a random walk until they hit the border of a drift zone around the internal gate of a pixel.

\subsubsection{Fast vertical signal charge collection}

As already explained, the position of electron hole pairs is fully determined by the Geant 4 simulation. Now, we will describe what happens to a small cloud of $n_{e}$ electrons created at the position $\vec{q}=(u, v, w)$ inside the silicon sensor. All computations are done in the local coordinates of the sensor and must be repeated for all electron clouds generated during step 1.

We can derive a parabolic potential model for a homogeneously doped $n$ type silicon between two fixed potentials at the front surface at $w=0$ and the back surface at $w=T$. For a fully depleted silicon bulk, the electrostatic potential $\varphi$ can be calculated from Poisson's equation

$$
\begin{gathered}
\partial_{w}^{2} \varphi(w)=-q N_{d} / \epsilon_{0} \epsilon_{S i} \\
\varphi(0)=\varphi_{\text {front }}, \quad \varphi(T)=\varphi_{\text {back }} .
\end{gathered}
$$

where $q$ is the electron charge, $N_{d}$ is the effective bulk doping and $\epsilon_{s i}$ is the relative permittivity of silicon. The solution is a simple parabolic potential distribution given as

$$
\varphi(w)=\frac{q N_{d}}{2 \epsilon_{0} \epsilon_{S i}} w(T-w)+\frac{w}{T}\left(\varphi_{\text {back }}-\varphi_{\text {front }}\right)+\varphi_{\text {front }} .
$$

with a potential minimum at a depth $w_{\min }$ below the sensor front side.

$$
w_{\text {min }}=\frac{T}{2}+\frac{\epsilon_{0} \epsilon_{S i}}{q N_{d} T}\left(\varphi_{\text {back }}-\varphi_{\text {front }}\right) .
$$

The $u-v$ plane inside the silicon sensor at $w_{\min }$ is the so-called potential valley. The $1 \mathrm{D}$ potential 


\section{The DEPFET detector simulation: Algorithm and its implementation}

model in Eq. 3.14 can be used to calculate the trajectory $w_{e}(t)$ of an electron cloud from the ionization point $w=d$ to the potential minimum $w_{\min }$ :

$$
\begin{gathered}
\frac{d w_{e}}{d t}=-\mu_{n} E_{w}, \quad E_{w}=-\partial_{w} \varphi \\
w_{e}(t=0)=d .
\end{gathered}
$$

Here, $\mu_{n}$ is the mobility of electrons in silicon at room temperature. The most important variable is the drift time $t_{d}$ needed to reach the potential valley $w_{\min }$. However, the naive drift time for approaching the potential valley $w_{e}(t) \rightarrow w_{\min }$ is infinite. Instead, we employ a truncated drift time to reach a silicon sheet

$$
w_{e}\left(t_{d}\right)=w_{\min } \pm \Delta
$$

around the potential valley with a thickness $\Delta$ of $3 \mu \mathrm{m}$. For electrons approaching the potential valley from the back, the drift time is given by

$$
t_{d}=\frac{\epsilon_{0} \epsilon_{s i}}{\mu_{n} q N_{d}} \ln \frac{d-w_{\min }}{\Delta}, \quad \text { if: } d>w_{\min }+\Delta
$$

Typical values for this drift time are in the order of $\sim 10 \mathrm{~ns}$ for $75 \mu \mathrm{m}$ thick DEPFET sensors. For electrons approaching the valley from the front surface, the drift time is given by

$$
t_{d}=\frac{\epsilon_{0} \epsilon_{s i}}{\mu_{n} q N_{d}} \ln \frac{w_{\min }-d}{\Delta}, \quad \text { if: } d<w_{\min }-\Delta .
$$

The drift time is important because it determines the amount of lateral diffusive spread of the electron cloud. An initially localized charge cloud with $n_{e}$ electrons will have a lateral spread at the potential valley that can be described by a $2 \mathrm{D}$ Gaussian charge density $\rho(u, v)$ with a width of

$$
\sigma_{u}^{D}=\sigma_{v}^{D}=\sqrt{2 U_{t h} \mu_{n} t_{d}}
$$

in the $u$ and $v$ direction. The thermal voltage $U_{t h}$ in silicon at room temperature is $26 \mathrm{mV}$. Without a magnetic field, the mean position of the charge cloud in the $u-v$ plane still equals the original position where the electron hole pair was created. In case of Belle II, a magnetic field of $B=1.5 \mathrm{~T}$ will point parallel to the local $v$ or row direction of the pixel modules. The Lorentz effect will shift the center of the charge cloud in the $u$ direction by an offset $\Delta_{L}$.

$$
\Delta_{L}=\left(d-w_{\text {min }}\right) \times \tan \theta_{L}
$$

When an electron cloud arrives at the potential valley, the electrons are split into groups of $\sim 50$ electrons and their positions $u_{0}, v_{0}$ are randomly sampled from the $2 \mathrm{D}$ density $\rho(u, v)$ to account for lateral diffusion during the drift time $t_{d}$.

The next step is to understand the relation between the $1 \mathrm{D}$ parabolic model calculation presented above to the real situation inside the DEPFET sensor. The doping concentration used for the simulation experiment is $N_{d}=10 \times 10^{12} \mathrm{~cm}^{-3}$. For a $75 \mu \mathrm{m}$ thin sensor, the potential at the back plane $\varphi_{\text {back }}$ is set to $-26 \mathrm{~V}$ relative to the source potential. Fig. 3.7 and 3.8 show a comparison between the $2 \mathrm{D}$ device 

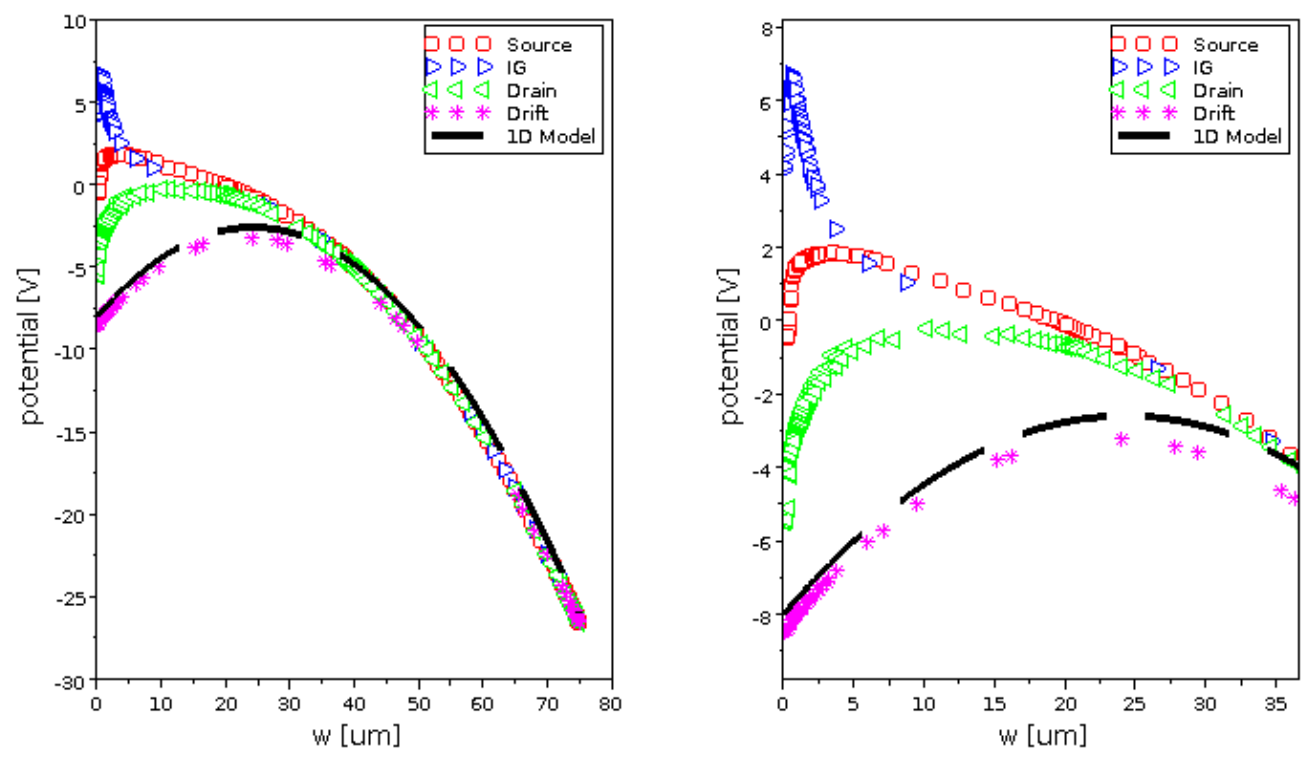

Figure 3.7.: Simulation of the potential $\varphi(w)$ for different positions on the pixel area. The dashed solid line is the simplified parabolic potential model.

simulation and the $1 \mathrm{D}$ parabolic model calculations. The agreement is reasonably good below the large area Drift and Clear regions at the pixel boundaries. The depth of the potential valley is $20 \mu \mathrm{m}$ below the front surface. The situation is more complex in the Source and Drain regions near the center of the pixel cell. The parabolic model is acceptable at positions below the potential valley but fails near the front surface. The potentials near the front surface strongly depend on the detailed doping profiles in these parts of the pixel cell. These lateral potential gradients are important for the lateral transport of electrons into the internal gate of a pixel cell.

\subsubsection{Lateral charge collection between pixels}

To understand the dynamics of electron transport by drift and diffusion in more detail, we will refer to a simplified $1 \mathrm{D}$ calculation. More precisely, we consider the transport of electrons in the potential valley along the $v$ axis using the Drift-Source cross section (see Fig. 3.5). The trajectory of electrons may be described by the stochastic drift diffusion equation

$$
d v=-\mu_{n} E_{v}(v) d t+\sqrt{\left(2 \mu_{n} U_{t h}\right)} d W(t)
$$

where $\mu_{n}$ is the electron mobility, $U_{t h}$ is the thermal voltage and $d W(t)$ Gaussian noise with mean zero. The variance of $d W$ is equal to the stepping time $d t$. A typical value for $d t$ used in the simulation is $0.5 \mathrm{~ns}$. The first term in Eq. 3.23 describes electron drift in an electric field while the second term describes electron diffusion as a Gaussian random walk. Near the Drift region between pixels at $v_{D}$, the lateral field $E_{v}$ is well described by a linear model:

$$
E_{v}(v)=\alpha_{D} \times\left(v-v_{D}\right)
$$



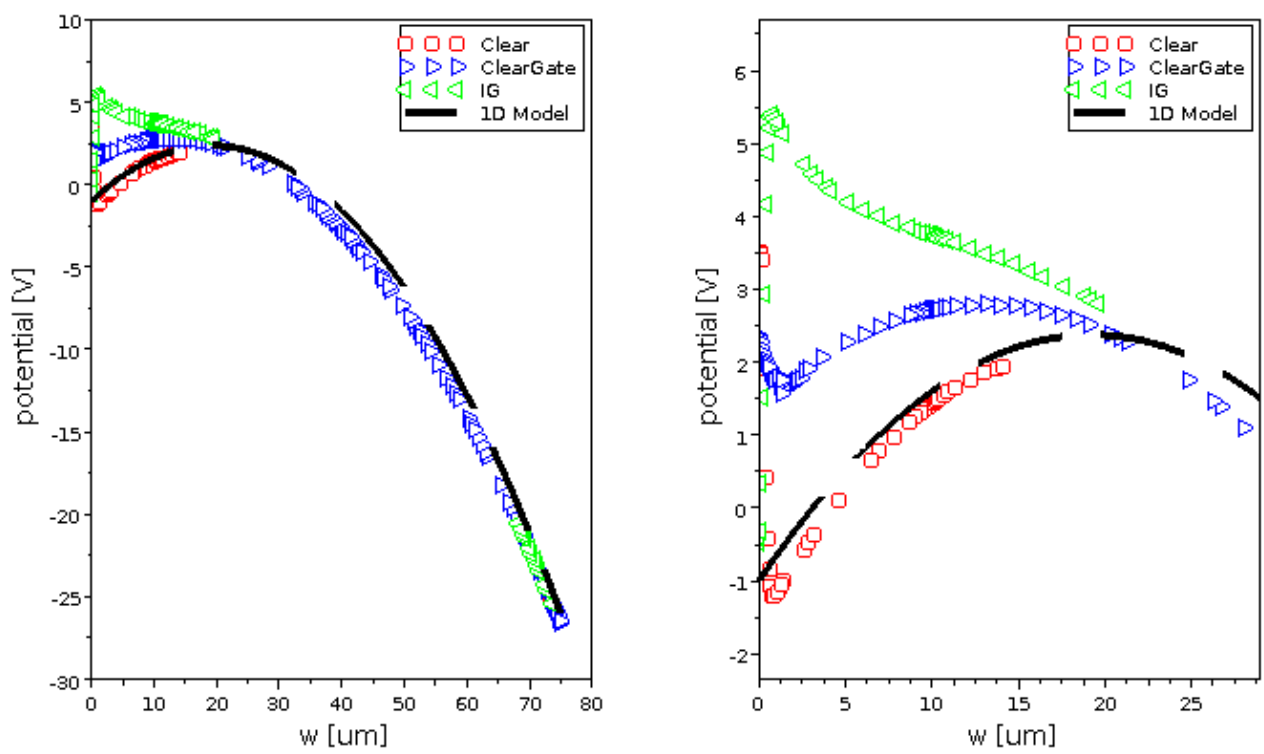

Figure 3.8.: Simulation of the potential $\varphi(w)$ for different positions on the pixel area. The dashed solid line is the simplified parabolic potential model.

The value of the field slope $\alpha_{D}=10^{5} \mathrm{~V} / \mathrm{cm}^{2}$ is extracted from the $2 \mathrm{D}$ simulations shown in Fig. 3.6. The transport model in Eq. 3.23 is used to compute the charge sharing probability $P(v)$. This probability is defined as the fraction of simulated trajectories where an electron starting at position $v$ finally reaches the internal gate of the upper pixel. The result of a toy simulation with 250 electron trajectories for each starting point is shown in Fig. 3.9. The charge sharing probability is $50 \%$ in the center of the Drift region at $v=v_{D}$ and increases almost linearly in the range $v_{D} \pm \Delta v_{D}$. The distance $\Delta v_{D}$ is called the border length of the Drift region. The probability $P(v)$ saturates at $100 \%$ for electrons starting at $v_{D}+\Delta v_{D}=9 \mu \mathrm{m}$. Since $P(v)$ is almost linear in the range $v_{D} \pm \Delta v_{D}$, we can approximate the drift-diffusion process by a bounded random walk in the range $v_{D} \pm \Delta v_{D}$. Once an electron crosses the border at $v_{D}+\Delta v_{D}\left(v_{D}-\Delta v_{D}\right)$, it drifts to the internal gate of the upper (lower) pixel. The details of this later process are not of interest for our problem as long as the electron reaches the internal gate. This simplification avoids an explicit modeling of the lateral drift fields between pixels and has an intuitive geometrical interpretation.

The extension of this concept for the relevant two dimensional case is shown in Fig. 3.10, The sensor area is divided into rectangular regions around the internal gate of pixels where drift dominates the electron transport. These drift regions are embedded in an area where diffusion dominates the electron transport. The length of the diffusion borders depends on the electric field slope at the Drift, Clear and Source border of the pixel. The simulation of vertical charge collection provides the $u, v$ coordinates for electron groups reaching the potential valley. These coordinates are the starting point for a two dimensional random walk in the $u, v$ plane. The random walk is performed in time steps of $0.5 \mathrm{~ns}$ and stopped as soon as the electron group hits a drift border.

In order to evaluate the stopping condition of random walks, we must locate the pixel centers on the $u-v$ sensor plane. We consider the typical case of a checker board grid of rectangular pixels. The 


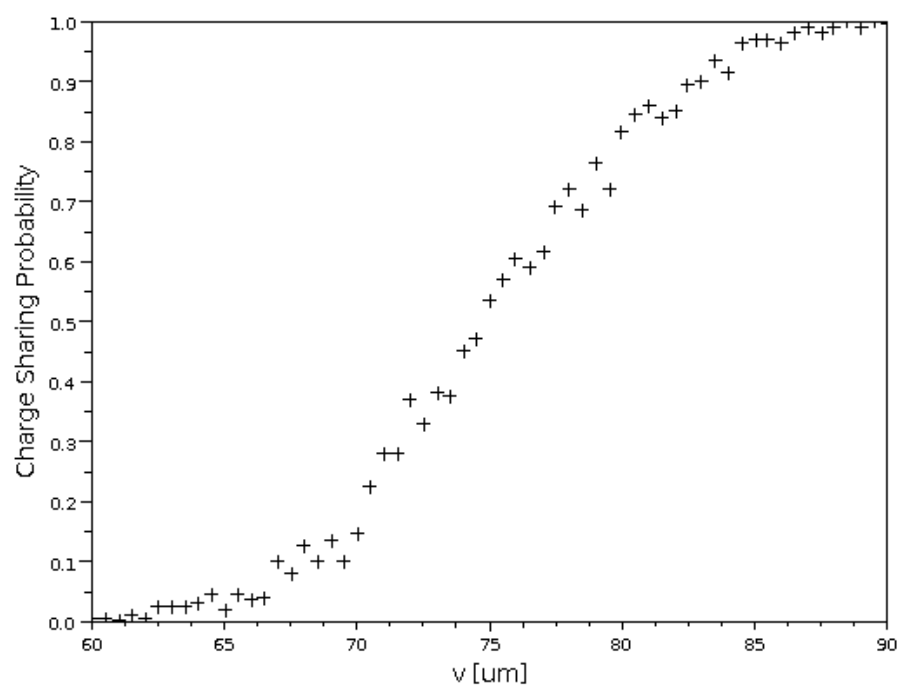

Figure 3.9.: Charge sharing probability $P(v)$ for signal electrons arriving at the potential valley $w_{\min }$ in near the Drift border at $v_{D}=75 \mu \mathrm{m}$ between two pixels. The lateral transport of electrons is dominated by diffusion in the range $v_{D} \pm \Delta v_{D}$. The border length $\Delta v_{D}$ is around $9 \mu \mathrm{m}$.

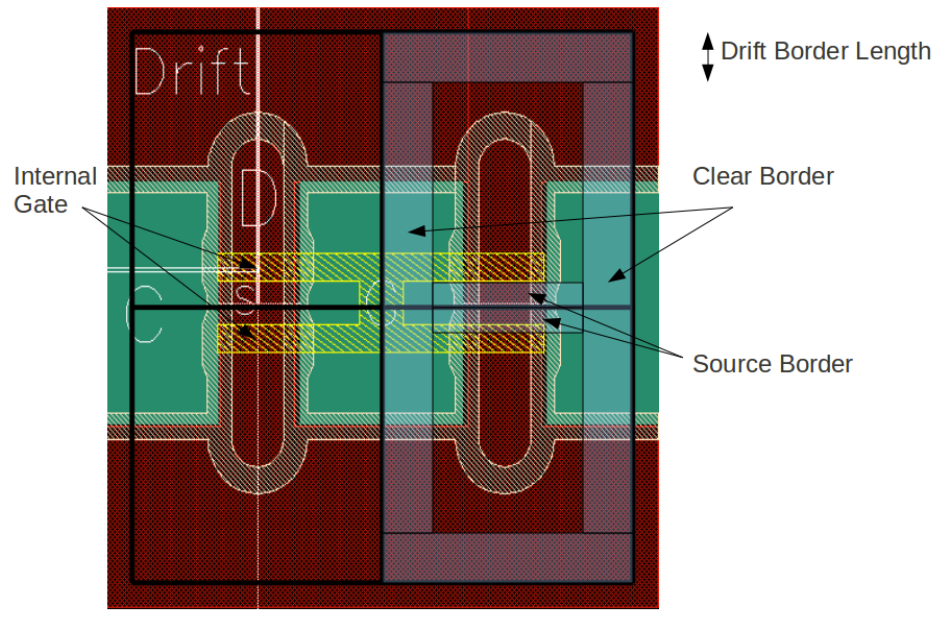

Figure 3.10.: Definition of Drift, Clear and Source border regions for a Belle II pixel cell. In these border regions, the lateral transport of signal electrons is dominated by diffusion. 
pixel cells will be counted from the lower left sensor corner by a column index $i$ and a row index $j$. The geometrical center of the pixel $i, j$ has the local coordinates

$$
\begin{aligned}
& u_{c}(i, j)=P_{u}\left(i-n_{u} / 2\right) \\
& v_{c}(i, j)=P_{v}\left(j-n_{v} / 2\right)
\end{aligned}
$$

where $P_{u}$ and $P_{v}$ denote the pitch of the pixel cell along the $u$ and $v$ directions and $n_{u}$ and $n_{v}$ are the total number of columns and rows on the sensor.

\subsubsection{Step 3: From collected signal charge to readout digits}

The result of step 2 is the division of all signal electrons on the internal gates of pixel cells. It is impossible for reasons of speed of simulation to simulate the charge amplification in the DEPFET transistor and the signal processing the readout chips in detail. Instead, we account for the readout step as a simple Gaussian smearing of the collected number of electrons in pixels. The Gaussian smearing is followed by a zero suppression step which discards very small signals.

The measurement of the number of electrons $Q_{i, j}$ in the internal gate of a pixel has two stages: a charge to current conversion in the DEPFET pixel transistor and a digital 8bit sampling of the transistor current arriving at the DCDB readout chips. The transistor current $I_{i j}=I_{b}+I_{n}+g_{q} Q_{i j}$ consists of a pixel specific baseline current $I_{b}$, a noise current $I_{n}$ and an amplified signal $g_{q} Q_{i j}$. The DCDB allows an analog $2 \mathrm{~b}$ correction of offset currents and has a dynamic range for corrected input currents of $24 \mu \mathrm{A}$ [38]. Further digital data processing in the DHP chips includes a digital offset correction to remove the baseline current and a zero suppression to discard too small signals. Both readout chips add noise to the detected signals. A detailed account of all steps is given in the Belle II technical design report ([11], chapter 4) were the readout of the pixel detector including the readout chips are explained in detail. For the simulation, we are forced to omit a number of steps for the analog and digital data processing. The short cut used is the following: after proper calibration, the DHP readout chip produces calibrated measurements of the collected number of electrons in the internal gate of DEPFET pixels. The simulation aims to provide readout digits at the level of the DHP outputs in order to speed up computations. As the study goal is an accurate prediction of the spatial resolution, we will focus on the overall electronics noise of the system and the zero suppression performed on the DHP readout chips.

\section{Equivalent noise charge}

We consider a pixel having collected $Q$ signal electrons. The signal is smeared by a Gaussian random number $\epsilon_{E N C}$ to account for readout noise. The readout noise has zero mean due to digital offset corrections and the standard deviation is the so-called equivalent noise charge (ENC). The ENC can be obtained from calibration measurements with real modules. The Belle II specification require an ENC in the range $100-200$ electrons. In the simulation, readout noise is added to all pixels which have a positive signal charge $Q$. The smeared pixel signal is

$$
Q^{s}=Q+\epsilon_{E N C}
$$




\section{Zero suppression and noise hits}

Only signals above the zero suppression threshold are maintained, all other signals are discarded. Zero suppression requires that the pixel signal $Q^{s}$ surpasses a so-called zero suppression threshold:

$$
Q^{z s}=\left\{\begin{array}{lll}
Q^{s} & , & Q^{s}>N_{z s} \times E N C \\
0 & , & Q^{s}<N_{z s} \times E N C
\end{array}\right.
$$

The threshold is expressed as a multiple $N_{z s}$ of the equivalent noise charge. After the zero suppression is applied to all pixels with true signal charge, the pixel matrix is populated with additional noise digits. The number of noise digits is computed as the mean number of pixel cells with a Gaussian readout noise $\epsilon_{E N C}$ above the threshold $N_{z s} \times E N C$. The noise digits are uniformly distributed over the sensor columns and rows.

\section{Readout frame}

The result of the simulation is a readout frame, a list of digits from all pixels with a signal $Q^{z s}$ above the zero suppression threshold. The readout frame contains signals from all particle hits within the sensor integration time. The beginning of the integration time is defined by the arrival of the trigger signal. For example, the number of hits per readout frame during a test beam experiment depends on the intensity of the particle beam. The digits in a readout frame are stored in an LCEvent as a collection of type TrackerData as described in section 3.2.3.

\subsection{A case study on spatial resolution of thin DEPFET sensors}

So far, we have outlined the steps to simulate digits from a DEPFET pixel detector in general terms; no particular sensor layout with a specific choice for the pixel sizes, the sensor thickness or the number of pixels was needed to be assumed. In this part now, we provide a particular example and simulate the readouts digits for a sensor design that was actually tested with the EUDET beam telescope in the years 2012 - 2013. This section simulates a particle beam hitting a DEPFET detector module and introduces a number of hit reconstruction variables that can be compared to real data.

First, we provide a summary of the simulation parameters that define a sensor layout for the detector simulation. Second, we proceed through the hit reconstruction steps from digits to hits. These steps are identical for real and simulated data and can be re-used for validation studies (chapter 5). Finally, we give the results for cluster sizes, signals and spatial resolution for a case study with a beam of $3 \mathrm{GeV}$ electrons hitting a pixel module at a tilt angle of 55 degrees. The case study shows how the simulation setup can be used to parametrize the intrinsic spatial resolution for different sensor layouts. The simulation setup will be re-used for the validation with many more test cases and the opportunity to compare the simulation results with real measurements.

\subsubsection{The setup of the simulation: Overview of parameters}

Tab. 3.1 summarizes the parameter values for the DEPFET detector simulations. The first column provides parameter values for the Belle II PXD modules extracted from the device simulation. The second column provides parameter values for the DEPFET modules used in test beams from the most recent 
3. The DEPFET detector simulation: Algorithm and its implementation

\begin{tabular}{|l|c|c|}
\hline & Belle II PXD & PXD6 \\
\hline \hline Sensor Thickness (in $\mu \mathrm{m})$ & 75 & 50 \\
\hline Pixel Pitch $\left(\right.$ in $\left.\mu \mathrm{m}^{2}\right)$ & $50 \times 75$ & $50 \times 75$ \\
\hline Pixel Noise $(\mathrm{ENC})$ & $100-200$ & 120 \\
\hline ZS Threshold $\left(N_{z s}\right)$ & 5 & 5 \\
\hline Bulk Doping (in $10^{12} \mathrm{~cm}^{-3}$ ) & 10 & 10 \\
\hline Back plane Voltage (in V) & -26 & -13.5 \\
\hline Drift Border Length (in $\mu \mathrm{m})$ & 9 & 9 \\
\hline Clear Border Length $($ in $\mu \mathrm{m})$ & 10 & 10 \\
\hline Source Border Length $($ in $\mu \mathrm{m})$ & 7 & 7 \\
\hline
\end{tabular}

Table 3.1.: Overview of DEPFET simulation parameters: The first column specifies parameter settings for Belle II PXD modules. The second column specifies parameter settings for the simulation of the most recent DEPFET prototype modules available for test beams.

sensor production (PXD6). The reduced thickness of the PXD6 sensors requires an adjustment of the back plane potential to $-13.5 \mathrm{~V}$ relative to the source potential. The distribution of lateral electric fields in the potential valley near the front surface is expected to be independent from the sensor thickness and the extracted values for length of border regions can be used for both designs. The equivalent noise charge measured during test beams is $100-120$ electrons and meets the requirements for Belle II. The calibration of the pixel noise from test beam measurements is discussed in section 5.4.1. The readout of signal electrons is parametrized by two parameters, namely the pixel noise and the zero suppression threshold. The pixel noise is given as an equivalent noise charge including all steps of analog and digital signal processing up the level of zero suppressed digits at the output of the DHP chip. The zero suppression threshold $N_{z s}$ is given as a multiple of the equivalent noise charge.

\subsubsection{Steps for data reconstruction}

For the simulation experiment, we shoot $50 \mathrm{k}$ electrons with an energy of $1 \mathrm{GeV}$ at a single PXD6 module. The simulation assumes a low beam intensity with one particle hit per event. The direction of the electron beam is parallel to the $z$ axis and the particle $x$ and $y$ positions at the $z=0$ plane are uniformly smeared by $5 \mathrm{~mm}$ to account for the beam size. The DEPFET sensor is rotated around the $x$ axis by 55 degrees. The simulation of particles in Mokka results in an LCIO file which stores the energy losses in $50 \mathrm{k}$ events. The DEPFET specific part of the simulation, steps 2 and 3 , are implemented in a dedicated Marlin Processor "DEPFETDigitizer" which adds a collection of zero suppressed digits to each event in the LCIO file. Two additional Marlin Processors "DEPFETSparseClustering" and "DEPFETHitMaker" are needed to reconstruct particle hits from digits. The matching between reconstructed and true hits as well as the sampling of position coordinate errors, cluster sizes and signals is handled in another Marlin Processor called "DEPFETSpatialResolution".

\subsubsection{From readout digits to clusters}

The starting point for clustering is the collection of digits that represent the readout frame of a detector module. The task of clustering is to process these digits and to form groups of digits from neighbouring pixels. These candidate clusters are believed to contain all ionization charge created from one particle. In order to discriminate cluster from particles against detector noise, only cluster candidates passing 
threshold cuts on the signal of the highest digit ("seed signal") and the sum of all digits ("cluster signal") are maintained for hit reconstruction.

In the following, a general method is introduced to compute candidate clusters from readout digits called sparse clustering. The sparse clustering algorithm builds on the concept of neighbouring digits: two digits are neighbours if they belong to pixel cells sharing a common edge or corner on the sensor. The advantage of this topological definition is, that it is independent of the pixel pitch and can be used for many sensor layouts. The sparse clustering method is implemented in the Marlin Processor DEPFETSparseClustering and can be used for particles with an arbitrary incidence angle.

\section{The sparse clustering algorithm}

We start with a non empty list of all digits from a detector module called HITS. Moreover, we maintain an initially empty list of candidate clusters called CLUSTERS. The first digit in HITS is assigned to a candidate cluster called CLUSTER[0] which is added to CLUSTERS. Now, we proceed to the second digit in HITS. In case this digit is a neighbour of the first digit, it is added to CLUSTER[0]. Otherwise it is added to a new cluster, called CLUSTER[1], which is in turn added to CLUSTERS. Now, we proceed to the third digit in HITS. We test if this digit is a neighbour of any digit in any candidate cluster previously added to CLUSTERS. If not, this digit opens a new candidate cluster which is added to CLUSTERS. If yes, this digit belongs to one or more cluster candidates in CLUSTERS. We add this digit to the first such candidate cluster and merge all other candidate clusters which are also adjacent.

\section{Signal cuts for cluster candidates}

All candidate clusters in the list CLUSTERS are checked if the seed signal and the total signal are above threshold cuts. The threshold cuts are:

- seed signal threshold given as $N_{\text {seed }} \times E N C$

- cluster signal threshold given as $N_{c l u} \times E N C$

Candidate clusters above both thresholds are added to an LCIO cluster collection and are saved for later hit reconstruction. Candidate cluster below one of the thresholds are discarded. The seed and cluster thresholds play a similar role as the zero suppression threshold and are used to discriminate against noise. The ordering of the three thresholds is $N_{\text {clu }} \geqq N_{\text {seed }} \geqq N_{z s}$ and typical values are $7>5>3$. This choice is motivated by the fact that $N_{z s}=3$ removes already most noise digits while keeping digits with small signal collected next to the seed pixel. The upper limit for $N_{c l u}$ depends on the ratio between the most probable cluster charge and the equivalent noise charge. Too high thresholds throw away signal charge and reduce the cluster size. In the worst case, all detected signals from a real particle are thrown away and the particle hit is not detected.

A good property is that the algorithm can deal with arbitrary cluster shapes. The sparse clustering method was used in a bachelor thesis [55] to reconstruct the path of Delta electrons created from $120 \mathrm{GeV}$ pions in $450 \mu \mathrm{m}$ thick DEPFET detector modules. Fig. 3.11 shows the reconstructed path of a Delta electron in the $u-v$ plane of the silicon sensor. 


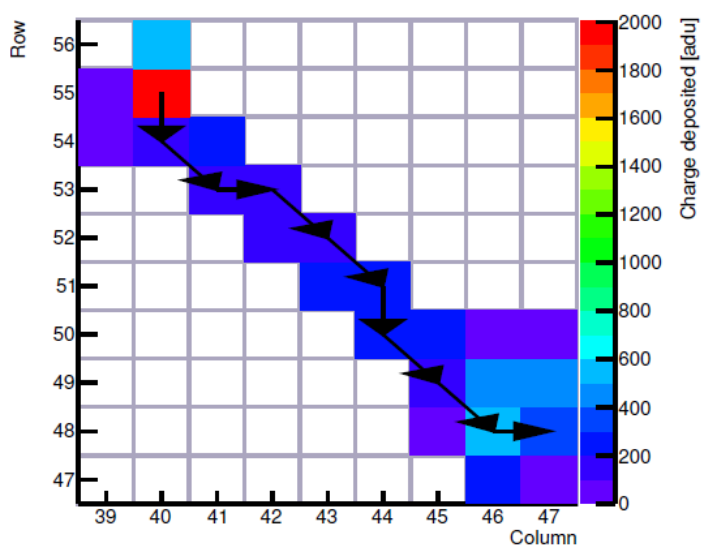

Figure 3.11.: Reconstructed path of a Delta electron in a $450 \mu \mathrm{m}$ thick DEPFET sensor emitted from a $120 \mathrm{GeV}$ pion [55]. The cluster is formed using the sparse clustering algorithm. The starting point of the Delta electron is tagged by the pion track detected in the EUDET telescope.

\subsubsection{From clusters to local hit positions}

The center-of-gravity algorithm is a robust method to compute where the particle has intersected the silicon sensor from a reconstructed cluster. The idea is to compute a weighted mean of the local pixel centers $u_{c}(i, j)$ and $v_{c}(i, j)$ of all pixels $i, j$ in the cluster, weighted with their detected charge $Q_{i, j}$. The estimated local coordinates for the hit positions relative to the $w=0$ plane of the silicon sensor are

$$
\begin{aligned}
& u_{m}=\frac{1}{\sum_{i, j} Q_{i, j}} \times \sum_{i, j} u_{c}(i, j) \times Q_{i, j} \\
& v_{m}=\frac{1}{\sum_{i, j} Q_{i, j}} \times \sum_{i, j} v_{c}(i, j) \times Q_{i, j}
\end{aligned}
$$

where the column and row numbers $i, j$ run over all digits in the cluster. To be useful for tracking and vertexing, measured hit coordinates $u_{m}, v_{m}$ must be accompanied with a $2 \times 2$ measurement covariance matrix $V$. The approach used here is to assume a diagonal covariance matrix and to parametrize the diagonal entries, the intrinsic spatial resolutions, as a function of the particle's incidence angle into the sensor. The intrinsic spatial resolutions are added to the Gear interface as explained in the appendix $\mathrm{C}$. This approach offers the advantage to fine tune the intrinsic spatial resolutions for any detector module in a test beam without additional programming effort.

\subsubsection{Sampling hit position errors}

The true particle hit can be calculated from the Geant 4 steps of the primary particle. A primary particle crossing a planar silicon sensor always creates two particular Geant 4 steps providing the entry and exit coordinates on the front and backside of the silicon sensor. The straight line connecting exit and entry point defines the local $u, v$ of the track intersection with the $w=0$ plane. The true hit coordinates are

$$
u_{t}=\frac{u^{e x i t}+u^{e n t r y}}{2}
$$




$$
v_{t}=\frac{v^{e x i t}+v^{e n t r y}}{2}
$$

A good property of this definition of the true hit coordinates is that it is purely geometric and independent of the energy deposits used for hit reconstruction. In particular, the true hit coordinates defined above are not affected by the production of secondary Delta electrons. The errors $\epsilon_{u}$ and $\epsilon_{v}$ of the coordinate measurements are defined as the distance between the reconstructed and the true intersection coordinates:

$$
\begin{gathered}
\epsilon_{u}=u_{m}-u_{t} \\
\epsilon_{v}=v_{m}-v_{t}
\end{gathered}
$$

Starting from a sample of tracks crossing the sensor, we can histogram the coordinate errors and estimate the probability density $\rho\left(\epsilon_{u}, \epsilon_{v}\right)$ of coordinate errors. The intrinsic spatial resolution is defined as the root mean square of the sampled coordinate errors.

$$
\begin{gathered}
\sigma_{u}=\sqrt{<\epsilon_{u}^{2}>} \\
\sigma_{v}=\sqrt{<\epsilon_{v}^{2}>}
\end{gathered}
$$

The correlation between $\epsilon_{u}$ and $\epsilon_{v}$ is typically very small for sensors with a checkerboard matrix hit by tracks at perpendicular incidence ${ }^{2}$. In the following, we assume that this correlation can be neglected. A more robust and useful estimator for the spatial resolution $\sigma_{u}$ is obtained by restricting the root mean error to the central $99 \%$ of the data points (RMS99). For tracking and vertexing with Kalman Filters, only the second moments of $\epsilon_{u}$ and $\epsilon_{v}$ are of interest, leading to a hit measurement covariance matrix

$$
V=\left(\begin{array}{cc}
\sigma_{u}^{2} & 0 \\
0 & \sigma_{v}^{2}
\end{array}\right)
$$

The intrinsic spatial resolution can be parametrized as a function of track variables. For minimum ionizing particles, track variables like the momentum $p$ and the charge $z$ have only a small impact on the energy loss in $50 \mu \mathrm{m}$ thin silicon sensors. The most important variables are the track incidence angle relative to the sensor plane and the in-pixel hit position.

\subsubsection{The results: cluster shapes and spatial resolution}

We present the results for a $3 \mathrm{GeV}$ electron beam incident on a $50 \mu$ m thick pixel module with $50 \times$ $75 \mu \mathrm{m}^{2}$ pixels (PXD6 layout in Tab. 3.1). The simulation is done without a magnetic field following the idea to stay close to the case of test beams with real modules where a magnetic field was not available. The electron beam is directed along the $z$ axis with a beam cross section of $5 \times 5 \mathrm{~mm}^{2}$ in the $z=0$ plane. The sensor is rotated by 55 degrees to produce elongated clusters in the $v$ direction (along the rows) while the clusters will be small in the $u$ direction (along the columns).

\footnotetext{
${ }^{2}$ In this case, the coordinate $u_{m}$ is estimated from pixels in adjacent columns while the $v_{m}$ coordinate is estimated from
} 


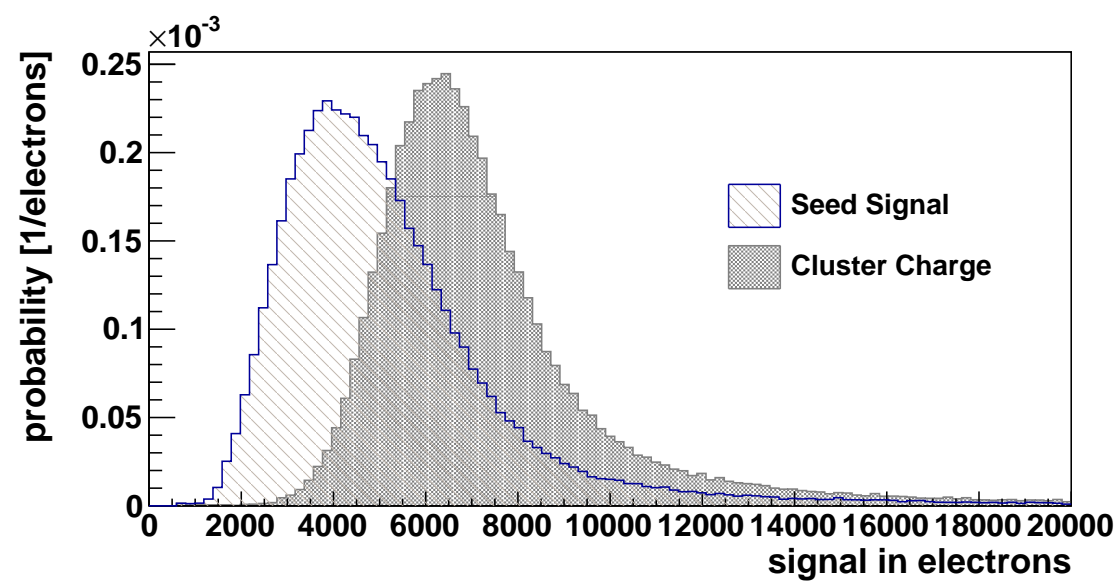

Figure 3.12.: Signal collected in clusters in response to $3 \mathrm{GeV}$ electrons at a tilt angle of 55 degree. The cluster charge is the sum of all signals in the cluster. The seed signal is the largest signal in the cluster. The most probable cluster (seed) charge is 6300 (4200) electrons.

The simulated detector response is shown in Fig. 3.12, The electrons create a signal of 6300 electrons (most probable value) in the silicon sensor. Roughly two thirds of the signal is contained in the pixel with the highest signal (the seed pixel), while the signal in the neighbouring pixels is much smaller. This means that a low zero suppression threshold is important to detect signal electrons in pixels around the seed pixel. For a zero suppression threshold of $5 \times 120$ electrons, the simulated cluster size is shown in Fig. 3.13. The most probable cluster has two hit rows and a single hit column. The cluster size of four hit pixels is possible when the particle passes between two columns.

The distribution of coordinate errors $\epsilon_{u}$ and $\epsilon_{v}$ is shown in Fig.3.14. A typical feature is the non Gaussian shape of the coordinate errors. The distribution of errors $\epsilon_{u}$ is box like reflecting the fact that mostly a single column responds to the particle. The so-called binary limit [56] describes a situation where only a hit in a single column is detected on the sensor. The predicted intrinsic spatial resolution in the binary limit $\left(P_{u} / \sqrt{12}\right)$ is $14.4 \mu \mathrm{m}$ and deviates significantly from the simulation result of $10 \mu \mathrm{m}$. The difference can be explained by the fraction of particle hits in the border region between two columns where charge sharing creates two column clusters. The intrinsic spatial resolution in the $v$ direction is $7.5 \mu \mathrm{m}$. The center of gravity hit reconstruction strongly benefits from the large fraction of two row clusters. The distribution of errors $\epsilon_{v}$ is not well described by a Gaussian but has strong tails. These tails are caused by large fluctuations of energy loss in different pixels of two row clusters. Large positive fluctuations of the energy loss in one pixel of the cluster inevitably pull the center of gravity towards this pixel. A more detailed analysis of the impact of energy loss fluctuations in thin silicon sensors on the intrinsic spatial resolution is given in [57].

The case study shows that the PXD6 design would be suitable for the forward region of the inner layer of the pixel vertex detector in Belle II. In the forward region of the first layer, charged tracks will hit the pixel sensor under an incidence angle of up to 60 degrees relative to the sensor normal. From the results shown above, we would expect to get mostly two row clusters with a spatial resolution of $<6.7 \mu \mathrm{m}$ for tracks in the $z$ direction of the Belle II coordinate system. For the column direction, we considered a worst case with minimal charge sharing and mostly one column clusters; still a spatial resolution

different pixels in adjacent rows. 

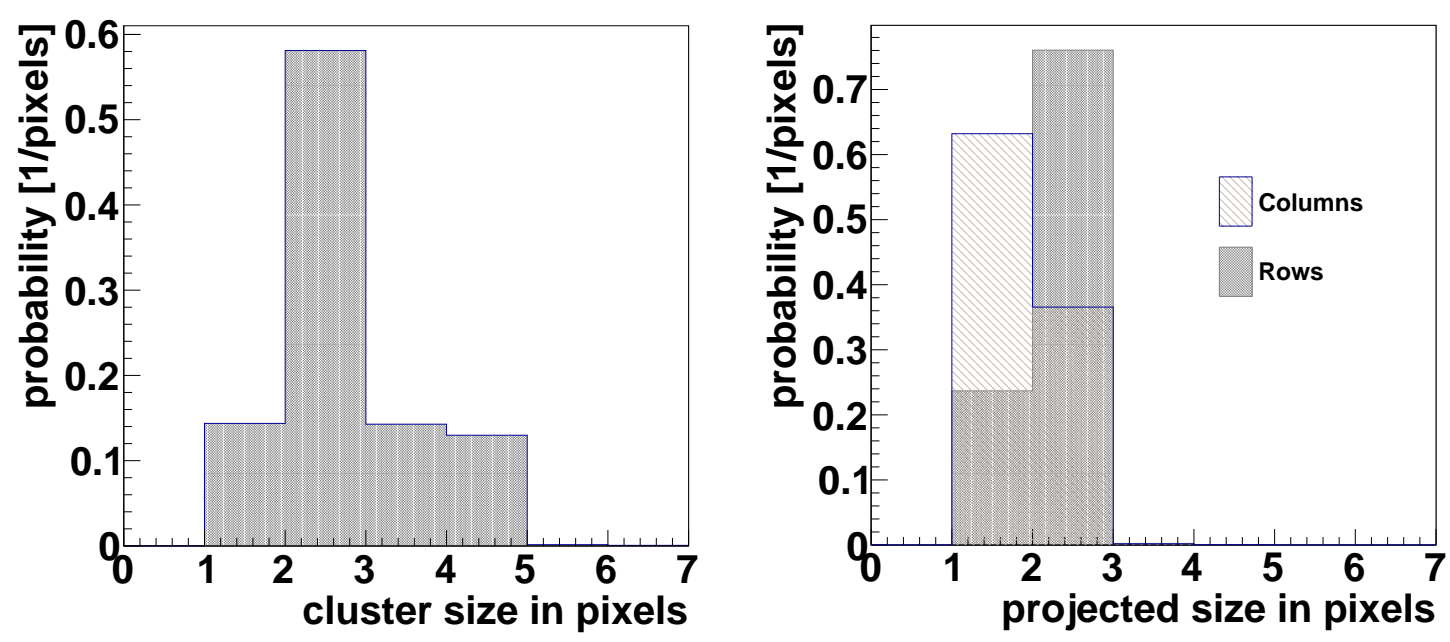

Figure 3.13.: The size of clusters from electron tracks measured by the number of hit pixels (left) or the number of hit columns and rows (right). Clusters are elongated in the local $v$ direction due to rotation of the sensor plane relative to the particle beam. In the $u-w$ plane, the tracks enter the sensor at normal incidence and mostly one sensor column is hit.
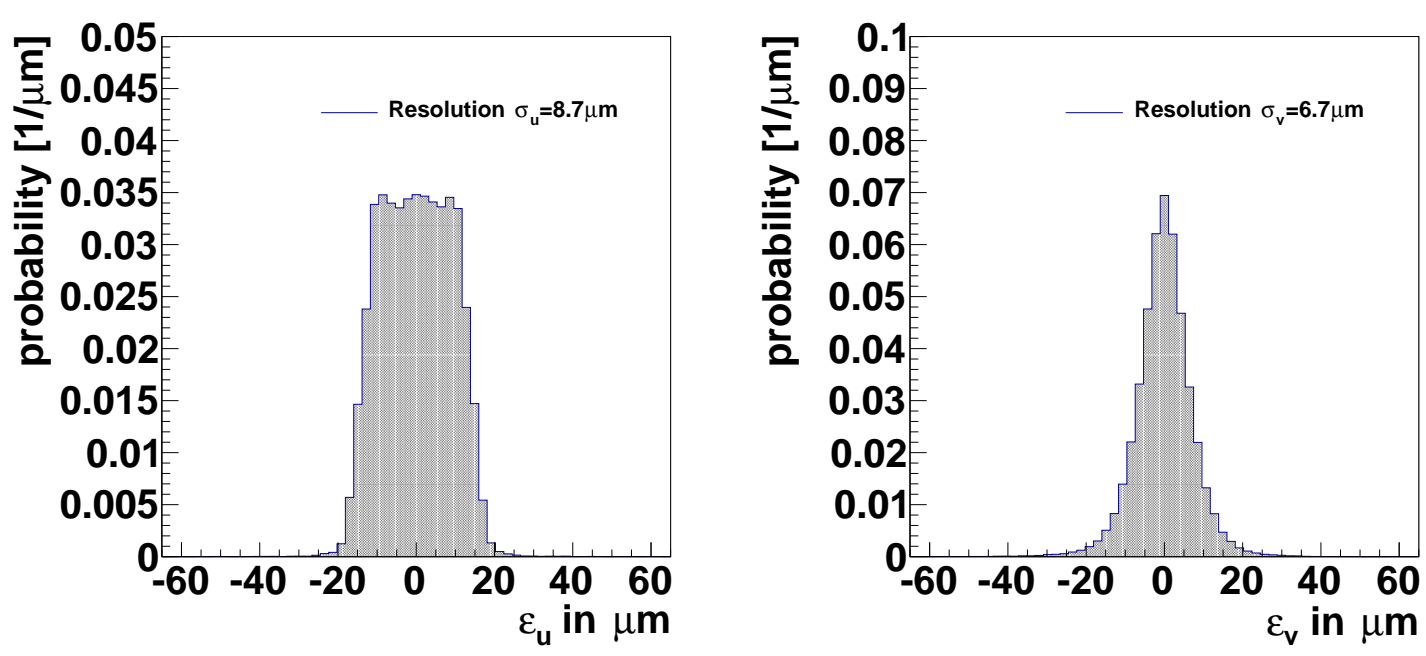

Figure 3.14.: Errors of hit coordinates $\epsilon_{u}$ (left) and $\epsilon_{v}$ (right) in the sensor $u-v$ plane. The spatial resolution is $8.7 \mu \mathrm{m}$ along the $u$ direction ( $50 \mu \mathrm{m}$ pitch) and $6.7 \mu \mathrm{m}$ along the $v$ direction ( $75 \mu \mathrm{m}$ pitch). The spatial resolution in the $v$ directions benefits from the tilted incidence of the beam particles on the sensor plane. 
of $8.7 \mu \mathrm{m}$ is obtained. As explained in section 2.2.2, a spatial resolution of $10 \mu \mathrm{m}$ in the innermost pixel layer is acceptable for Belle II because the vertex resolution is ultimately limited by multiple scattering. In the Belle II environment, we can expect additional charge sharing between columns from the Lorentz shift of charge clouds in the $1.5 \mathrm{~T}$ magnetic field with an accompanied improvement of the spatial resolution in the column direction.

As a summary, the design difference between relevant sensor layouts are well accounted for in the detector simulation. Once the simulation algorithm is validated from test measurements, we can be confident that the extrapolation of the spatial resolution to the design variations used in the first and second layer of the pixel vertex detector at Belle II are reliable. 


\section{The reconstruction of beam telescope data: methods, steps and calibration}

As already outlined in chapter2, it is necessary to validate the results of the detector simulation presented in chapter 3 in another work stream: As the EUDET beam telescope (already described in 2.3.2) does not provide us with the information required for the validation, especially regarding the intersection point at the device under test, we need to develop further methods for the reconstruction and calibration.

The results of this reconstruction will then be used for the real validation of the detector simulation (chapter 5). The goal is to compare digits from prototype DEPFET modules with the estimated track intersection points from the telescope. The situation closely parallels the simulated case study presented in the last section; the only difference is that the particle intersection point is now derived from telescope digits instead of the simulated energy losses.

The scope of this chapter is to develop and explain the reconstruction of the beam telescope data. It is organized as follows: Firstly we provide a brief overview of the reconstruction and calibration steps to obtain fully reconstructed tracks in a well aligned beam telescope. Secondly, we present the data model needed to work with reconstructed tracks and telescope alignment. Then, we follow the three main steps of the data reconstruction and present the methods for hit reconstruction, track fitting and telescope alignment in a more detailed way. In order to focus on important conceptual points, mathematical details of the developed methods for track fitting and alignment are presented in appendices.

\subsection{Overview of reconstruction and its challenges}

Simulations offer the possibility to study the response of a simulated pixel module to simulated particle hits. In particular, case studies like those presented in section 3.4 provide a means to study the intrinsic spatial resolution of pixels as a function of their design parameters and particle properties like its momentum or angle of incidence. The EUDET beam telescope offers the possibility to carry out very similar case studies with real particles and real sensors. Now, the readout digits from the telescope layers are used to compute an extrapolated particle intersection with a silicon sensor placed as a device under test in the center. The experimental challenges to be solved are threefold:

- Firstly, the estimation of track intersections (track fitting) has to take into account the effect of Coulombic (or multiple) scattering of the particle in the beam telescope. This is particularly necessary for data taking at DESY with $1-6 \mathrm{GeV}$ electrons where multiple scattering at the Mimosa26 sensors dominates the estimation errors. For a validation of the detector simulation, the estimation errors have to fall below $\sim 10 \mu \mathrm{m}$, which is the expected spatial resolution of the DEPFET pixel module for Belle II.

- Secondly, the track fitter should allow an estimation of track intersections on sensors with arbitrary 
$3 \mathrm{D}$ rotations. As seen in the case study, the intrinsic spatial resolution strongly depends on the incidence angle and the device under test must be tilted with respect to the beam axis by up to $\sim 55$ degrees.

- Thirdly, the positions and rotations of all sensors in the telescope, reference sensors and devices under test, must be calibrated from track data (telescope alignment). The generic difficulty for alignment is to deal with global distortions of the telescope and to achieve an accurate estimation of the angle of incidence into the sensors on the level of $\sim 1$ milliradians.

To tackle these problems, in this thesis we have further fine tuned the usual three step approach to reconstruction, with special regard to track fitting and telescope alignment with Kalman Filters. The three steps are:

Reconstruction of Clusters and Hits: In this step we proceed sensor by sensor from readout digits to hits. The step is performed by three Marlin Processors for the masking of hot pixels (DEPFETHotPixelKiller), the clustering of unmasked digits (DEPFETSparseClustering) and the estimation of local particle intersection coordinates from clusters (DEPFETHitMaker). The later two processors were already covered in the previous chapter and can be re-used.

Track finding and fitting: The task of track finding is to find hits that originate from the same particle at different telescope layers. The DEPFETFastTracker processor allows track finding at all telescope layers including hits from the devices under test. After finding those hits, a track fitting is performed to estimate intersection coordinates and incident angles at all sensors crossed by the particle. Unlike clustering, track finding and especially track fitting depends on a precise assessment of the position and rotation of all sensors in the telescope.

Telescope alignment: Alignment is the data driven calibration of sensor positions and rotations within a data taking period. The telescope geometry data base contains the geometry constants which are needed to define the transformations between local sensors and global telescope coordinates. The result of the alignment is an update of the telescope geometry data base which helps to improve track finding and fitting.

The reconstruction and alignment of beam telescope data typically requires to chain the above described steps as shown in Fig.4.1. Before we move into a detailed step by step explanation, we briefly describe the framework used for the study and the required data inputs from the EUDET telescope.

\subsection{The reconstruction framework: From digits to tracks}

\subsubsection{High energy particle beams at DESY and CERN}

The EUDET beam telescope is operated at particle beam lines which supply intense beams of strongly collimated high momentum particles. For this thesis, measurements with the EUDET beam telescope were performed by using particle beams at DESY and CERN. A summary of the most relevant properties of the two beam lines used during the measurement campaigns is given in Tab.4.1. The beam spot size is defined as the root mean square (RMS) of the particle density in a plane perpendicular to the beam direction while the particle rate gives the total particle flux per second through this plane.

In practice, the most important differences between the beam sites are the beam momentum and the particle rate. A high and continuous particle rate allows to quickly accumulate large samples of particle 


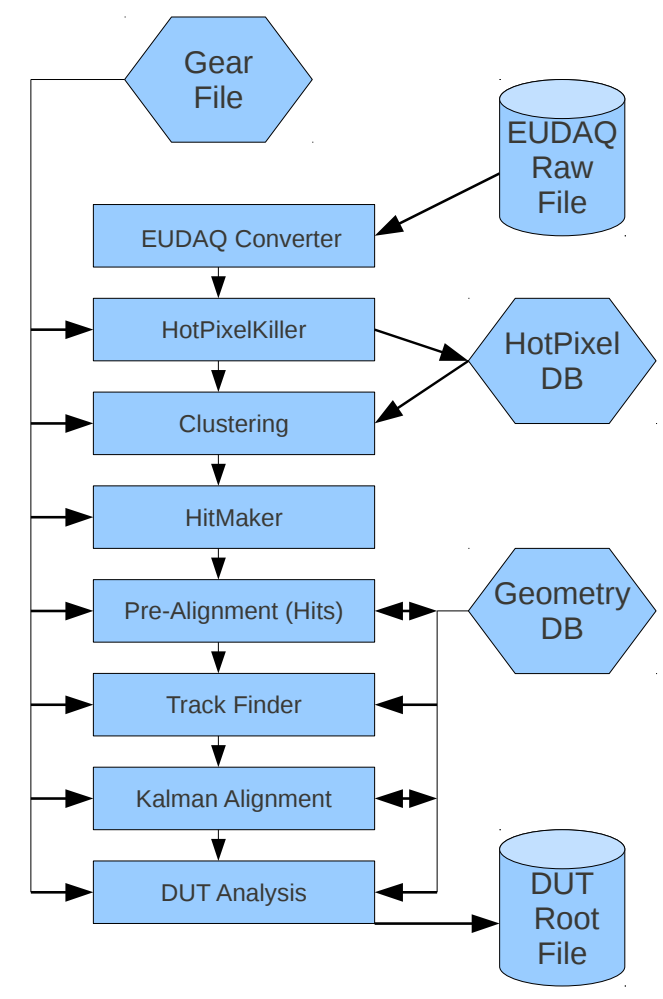

Figure 4.1.: Steps for data reconstruction and calibration for run data from the EUDET telescope: Firstly, the run file is converted into the LCIO format. Secondly, all events are processed step by step in a stream of Marlin Processors. The gear file provides the initial telescope geometry information needed for local hit reconstruction. Calibrated geometry constants for track finding and fitting are accessible from a geometry data base. In the final step, track intersections and hits at the devices under test are written to a Root file for user analysis.

\begin{tabular}{|l|c|c|}
\hline & DESY Test Beam & CERN SPS H6 \\
\hline \hline Particle Type & electrons/positrons & hadrons (mostly pions) \\
\hline Momentum $[\mathrm{GeV}]$ & $1-6$ & 120 \\
\hline Spot Size $[\mathrm{mm}]$ & $5-10$ & 5 \\
\hline Particle Rate $[\mathrm{kHz}]$ & $0.1-5$ & 1 \\
\hline
\end{tabular}

Table 4.1.: Overview of beam parameters at DESY and CERN. At DESY, the beam is continuous and beam parameters can be varied in the prescribed ranges. The H6 beam area at CERN SPS supplies beams of $120 \mathrm{GeV}$ pions. The beam is discontinuous and the quoted value is the maximum rate during a particle spill. 


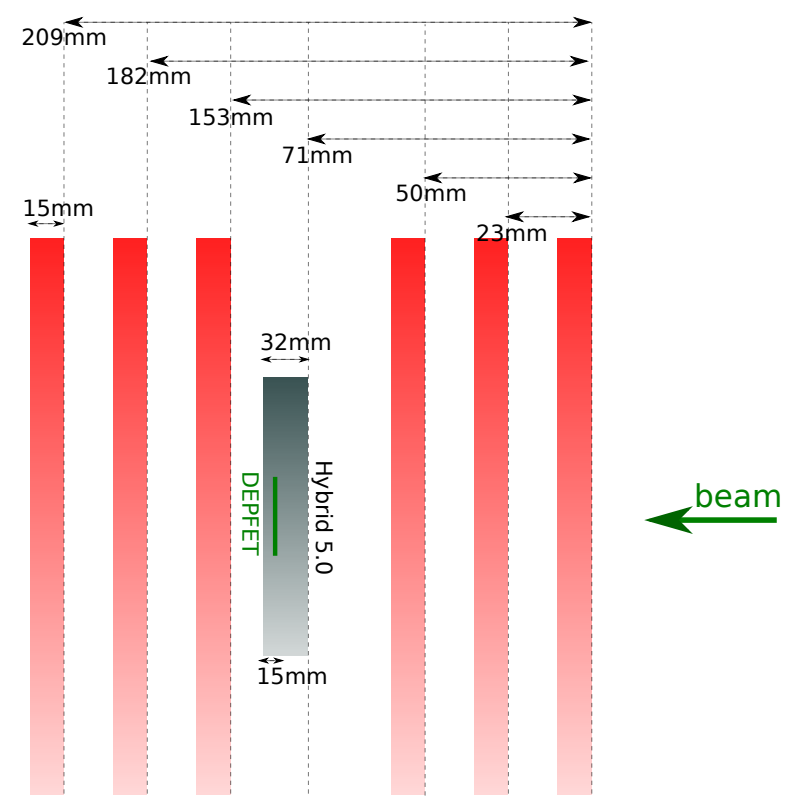

Figure 4.2.: Typical setup for a DEPFET test beam run with the EUDET telescope at DESY. The distance between the two telescope arms is around $100 \mathrm{~mm}$ limited by the space needed to install the DEPFET sensor box.

tracks needed for detailed detector studies, for example the study of in-pixel charge sharing presented in chapter 5. The large impact of the particle momentum on the resolution of track extrapolation was already discussed in section 2.2.2 for the case of the $z$ vertex resolution at Belle II.

At DESY [58], multiple test beam lines are fed by the lepton synchrotron DESY II. It provides up to $7 \mathrm{GeV}$ positrons, which are partially converted to a photon beam via Bremsstrahlung when hitting one or more carbon fiber targets close to the beam pipe perimeter. This photon beam in turn hits a conversion target (metal plate) and electron/positron pairs are created via pair production. A magnetic field spreads these beams into two fans with different directions. A collimator acts as a selector of particles of a certain momentum. The user has the option to vary the strength of the magnetic field. This results in particles of a different momentum passing through the collimator. That way the beam momentum and the intensity of the particle beam entering the telescope can be selected. The momentum resolution for test beams at DESY is around $1 \%$.

The testing of the DEPFET prototypes at the CERN Super Proton Synchrotron (SPS) beam line H6 was performed mainly in a beam of charged pions at $120 \mathrm{GeV}$. Every 40 seconds protons are extracted from the SPS ring accelerator. This so-called spill is collided with a wolfram target. The shower of secondary particles, mostly pions, is focused and channeled to the beam test area in the H6 beam area at the CERN Prevessin site. The length of a spill is 10 seconds and the particle rate during the spill is $\sim 1 \mathrm{kHz}$. The momentum resolution of the pion beam is $1 \%$.

A typical setup for a DEPFET test beam at DESY taken during the 2013 measurement campaign is shown in Fig.4.2. During this chapter, we will use a $3.75 \mathrm{GeV}$ electron run taken in this setup to illustrate the steps to reconstruct and calibrate test beam data. 


\subsubsection{The EUDET beam telescope as data source}

For the purpose of data reconstruction, it is important to clearly define the structure of data. Here, the interest is laid on the organization of the telescope data as it is the starting point for the event reconstruction. The key concepts for the organization of data are runs, events and sub-events. A run consists of a large number $(>10 \mathrm{k})$ of events collected under identical conditions with respect to telescope geometry and beam parameters. An event is a data container for all readout digits sent to the data acquisition system of the EUDET telescope, called EUDAQ [59, 60], in response to a particle trigger. In other words, the event contains the digits from one or more particles traversing the beam telescope. An event has a substructure and typically contains two sub-events. One sub-event contains the digits from all six Mimosa26 reference layers while the other contains the digits from the device under test; in our case a single DEPFET pixel module.

The ILCSoft framework requires the input data to be stored in the LCIO format. For this reason, the data should be converted from the native EUDAQ format into the LCIO data format. EUDAQ provides a data converter program that writes the sub-event data to LCIO::TrackerData collections in the LCIO file. The name of the collection identifies the name of the EUDAQ sub-event uniquely. The data reconstruction starts from these digit collections and subsequently adds more LCIO collections to the data file with clusters, hits and finally reconstructed tracks.

For the reconstruction of particle tracks in the EUDET beam telescope, we have to deal with additional complexity: the reconstruction of particle tracks depends on the telescope geometry, but the telescope geometry must be adjusted at the beginning of a run period, as the initial assumption about the geometry from mechanical measurements is not precise enough. The problem originates from the observation that the intrinsic spatial resolution of a telescope layer relative to the silicon sensor is $\sim 1 \mu \mathrm{m}$ while the positioning of the sensors in the laboratory is far less precisely known $(\sim 100 \mu \mathrm{m})$. The fine calibration of the sensor position and rotation in the laboratory is called telescope alignment.

Coming from here, the requirements for a proper data management for reconstruction are the following:

- Allow dynamic adjustment of the telescope geometry while maintaining a common interface to geometry data for reconstruction algorithms.

- Store event data (clusters, hits and tracks) in the LCIO file in a way that is independent from telescope geometry to avoid inconsistencies after geometry changes.

To fulfill the first requirement, we re-use the telescope geometry model developed in section 3.2 .2 for the detector simulations. Especially, the TBDetector class serves as a common interface to retrieve all geometry related data for track fitting and alignment. The TBDetector class implements an interface to retrieve geometry data from the gear file and a separate geometry data base. The geometry data base allows to persistently store those constants of the geometry model which must be calibrated during the telescope alignment. The geometry data base contains the following data for silicon sensors 1 .

- $k$ : The sensor or layer number along the beam line

- $x_{0}, y_{0}, z_{0}$ : The origin of the sensor center in telescope coordinates

\footnotetext{
${ }^{1}$ Discrete rotations need not be updated and are not included in the data base.
} 
- $\alpha_{0}, \beta_{0}, \gamma_{0}$ : The tilts of the silicon sensor in telescope coordinates

These geometry constants define the transformation between local and global telescope coordinates. The geometry data base gets initialized from the telescope geometry described in the gear file. It can be updated in a number of alignment steps and can be used in all reconstruction steps which rely on accurate track fitting.

For the second requirement, the proper storage of event data, the method developed in this thesis is the following: For hits, the decoupling from the telescope alignment constants is possible by storing only local hit coordinates and their covariance in the LCIO file; for details see the event data model in 3.2.3. For reconstructed tracks, the solution is to exclusively store the hit-to-track assignments in the LCIO events. Essentially, the LCIO::Track class used for the persistent storage of tracks only contains the list of hits found along the particle's track and the particle hypothesis. The particle hypothesis contains the mass $m$, charge $q$ and momentum $p$. An additional helper class is designed to combine the local hits stored in the LCIO::Track with the current telescope geometry data on the fly. By doing so, it is possible to quickly refit tracks at all stages of the data reconstruction using the most precise geometry constants. This approach avoids to make changes to the digits, clusters, hits and reconstructed tracks stored in the LCIO run file after alignment corrections are applied to the geometry data base. For user analysis, a final Marlin Processor is developed to write a Root [61] file containing the estimated track parameters at the devices under test after the reconstruction and calibration steps are finished.

\subsection{The reconstruction of clusters and hits (Step 1)}

The reconstruction steps to proceed from digits to clusters and from clusters to hits have already been discussed in section 3.4.2 for the case of a single DEPFET module in the beam line. There, the hit reconstruction was organized in two Marlin Processors called DEPFETSparseClustering and DEPFETHitMaker. For the reconstruction of telescope events, these two processors should be run separately for the Mimosa26 and DEPFET sub-events. In this way, it is possible to use the same algorithms but with adjusted steering parameters for digits from DEPFET and Mimosa26 modules. A further pre-processing step is needed to allow a masking of "hot" pixels on the Mimosa26 and DEPFET modules.

\section{Hot pixel masking}

The DEPFETHotPixelKiller is a Marlin Processor designed for data driven and automatic masking of hot pixels in a specified run. The input is the collection of digits and the output is a conditions data base (HotPixelDB) containing a list of masked or hot pixels to be used in the clustering processor. The processor computes a digit rate $f$ as the ratio of the number of detected digits from a pixel divided by the number of processed events. The expected rate $f$ is small and mostly depends on the chosen threshold for zero suppression. Assuming Gaussian noise, a zero suppression threshold of $N_{z s}=5$ implies that only in 1 event out of $10^{6}$ the readout noise will be high enough to fake a particle hit. However, if a pixel exceeds this threshold too often, it means that there is something weird going on and we better mask it. As can be seen in Fig.4.3, a few "hot" pixels have much higher rate than the rest and will be masked for further data processing. The hot pixel threshold is set to $f \geqq 10^{-3}$. The typical number of hot pixel per Mimosa26 module is in the order of $<100$ pixels which should be compared to the total number of $600 \mathrm{k}$ pixels per module. 

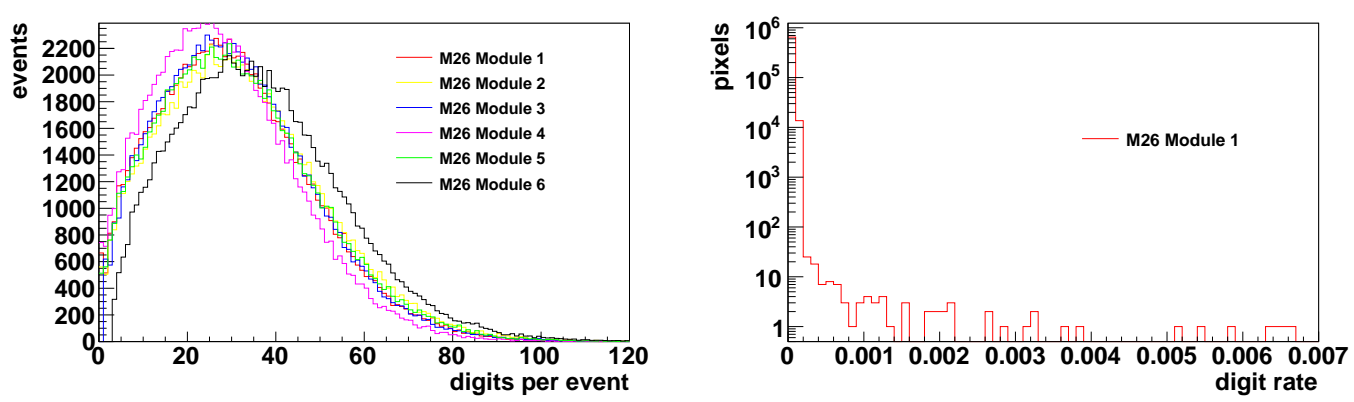

Figure 4.3.: Left: Histogram of the number of firing digits per event for all six Mimosa26 modules. The most probable number of digits per event is $20-30$ but events with up to 100 digits are recorded. Right: Histogram of digit rates for a Mimosa26 module in the same run. The digit rates are computed from $80 \mathrm{k}$ events and the hot pixel threshold is $10^{-3}$.

\begin{tabular}{|l|c|c|c|c|c|c|c|}
\hline \multirow{2}{*}{ Module } & \multicolumn{2}{|c|}{ number of pixel } & \multicolumn{2}{c|}{ pitch $[\mu \mathrm{m}]$} & \multicolumn{2}{c|}{ resolution $[\mu \mathrm{m}]$} & area $\left[\mathrm{mm}^{2}\right]$ \\
\cline { 2 - 8 } & in $u$ [cols] & in $v$ [rows] & in $u$ & in $v$ & in $u$ & in $v$ & $(u \times v)$ \\
\hline \hline Mimosa26 & 1152 & 576 & 18.4 & 18.4 & $3.5-4.5$ & $3.5-4.5$ & $(21.2 \times 10.6)$ \\
\hline DEPFET & 32 & 64 & 50 & 75 & 10 & 18 & $(1.6 \times 4.8)$ \\
\hline
\end{tabular}

Table 4.2.: Overview of sensor data for hit reconstruction: The layout of the pixel matrix is parametrized by the pixel pitches in the $u$ and $v$ directions and number of pixels. For Mimosa26 modules, the spatial resolution depends on the zero suppression and detector noise level. For the tested DEPFET modules, the quoted value is the expected spatial resolution for perpendicular incidence of tracks.

\section{Clustering and HitMaker}

The clustering of data from the DEPFET module with the DEPFETSparseClustering processor has already been discussed before and can be re-used. The same clustering processor can also be applied to the digits from Mimosa26 modules. The detected signal in Mimosa26 digits is a digital ' 1 ' indicating the detection of a signal over threshold. This means that the threshold cuts on the signals in candidate clusters are always set to zero in the clustering procedure. In other words, candidate clusters are always accepted. In order to avoid an excessive number of clusters from hot pixels, the DEPFETSparseClustering processor loads the HotPixelDB and ignores digits from hot pixels. This hot pixel suppression in the clustering reduces the number of reconstructed clusters per Mimosa26 module by a factor of $1.5-2.0$.

The DEPFETHitMaker can be used to process clusters from DEPFET or Mimosa26 modules without additional adjustments. The tested DEPFET modules and Mimosa26 modules both have a checker board matrix of pixel cells. The relevant geometrical parameters are given in Tab.4.2. These parameters allow to compute the center of pixel cells on the local $u, v$ measurement plane and specify the diagonals of the hit covariance matrix $V$.

For clusters on Mimosa26 modules, all digits are equally weighted in the center of gravity estimator for the hit intersection coordinates 2 For the relevant case of perpendicular incidence of tracks, the optimal spatial resolution is obtained when cluster sizes are small and only digits from a field of $2 \times 2$ pixels around the particle intersection contribute. In this case, there are only three types of clusters for Mimosa26 modules: The first case (case a) are one pixel clusters and the second case (case b) are two

\footnotetext{
${ }^{2}$ The detected signal is a digital ' 1 '.
} 

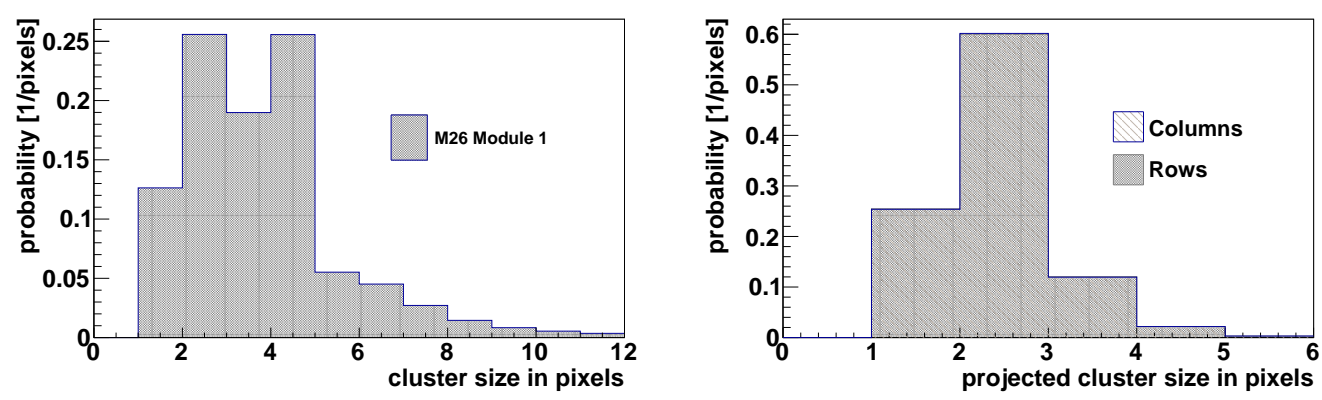

Figure 4.4.: Left: The size clusters on a Mimosa26 module in response to electrons at perpendicular incidence. The size one bin gives the number of single pixel clusters (case a). The peaks at sizes of two and four result from clusters of case b or case c. Large clusters with more than four pixels are considered to be outliers and should be ignored for track fitting. Right: The projected cluster size on columns and rows are nearly identical since the pixel pitch is equal in the $u$ and $v$ direction of the sensor. The large fraction of clusters with two columns (rows) shows significant charge sharing between neighboring pixels.

pixel clusters having pixels from two adjacent rows or columns. In the third case (case c), we have clusters with three or four pixels from two adjacent rows and two adjacent columns. Clusters with more than four pixels are suspicious and are most likely contaminated by noise digits. The distribution of cluster shapes on Mimosa26 modules for a test beam at DESY with $3.75 \mathrm{GeV}$ electrons is given in Fig. 4.4 .

The intrinsic spatial resolution of Mimosa26 modules depends on the distribution of cluster shapes which in turn depends on the zero suppression threshold. For a very high threshold, clusters are expected to contain only a single pixel (case a) and the spatial resolution in the $u$ and $v$ directions will be given by the binary limit formula [56]:

$$
\sigma_{u, v}=P_{u, v} / \sqrt{12}
$$

A lower threshold leads to larger clusters due to charge sharing and has the potential to improve the spatial resolution beyond the binary limit as argued in [62]. The basic idea is that a cluster with signal in two adjacent rows indicates a particle hit near the edge between the rows while a one row cluster signals a hit near the row center. In the ideal case, a $1: 1$ ratio between single and double row (or column) clusters can be reached and the row (column) pitch is effectively halved. In this case, the spatial resolution for sensors with purely digital readout is expected to be given as [62]:

$$
\sigma_{u, v}^{\star}=P_{u, v} / 2 \sqrt{12}
$$

The Mimosa26 sensors have a pitch $P_{u, v}$ of $18.4 \mu \mathrm{m}$ and their spatial resolution should be somewhere in the range from $2.7 \mu \mathrm{m}$ (best case) to $5.4 \mu \mathrm{m}$ (worst case). Reported numbers in the literature range from $3.5 \mu \mathrm{m}$ to $4.5 \mu \mathrm{m}$ but the dependence on the zero suppression threshold and the probability of outliers are not explicitly studied [41, 59]. A more reliable approach is a data driven calibration of the spatial resolution of Mimosa26 sensors from track data [63]. For this thesis, we will use a number of $3.5 \mu \mathrm{m}$ for the spatial resolution of Mimosa26 modules for test beams at DESY and CERN. In section 4.4.2.1, we will demonstrate that this number is statistically consistent with track residuals obtained for 
a test beam with $3.75 \mathrm{GeV}$ electrons at DESY.

\subsection{Track finding and fitting (Step 2)}

After having performed step 1, telescope events contain reconstructed hits on all pixel layers along the particle beam. A typical telescope event contains more than one hit per layer caused by multiple beam particles traversing the telescope during the integration time of pixel module or additional readout noise leading to fake hits. Track finding is a pattern recognition or classification problem and aims at dividing the set of hits in a telescope event into subsets, each subset containing measurements believed to originate from the same particle. These subsets are called reconstructed tracks. In track fitting, the hits in reconstructed tracks are used to estimate the intersection coordinates and incident angles of a particle as it traverses the silicon sensors in the telescope.

\subsubsection{A fast and combinatorial track finder}

The DEPFETFastTracker provides a fast and generic solution for track finding in the EUDET beam telescope. The processor reads input hits from one or more hit collections in the LCIO file. The approach chosen here allows a simultaneous track finding in all telescope layers including one or more devices under test. The output of the DEPFETFastTracker is a collection of reconstructed tracks stored in the LCIO file for usage in track based telescope alignment and validation studies. The processor reads the initial telescope geometry from the gear file but updates layer positions and rotations from an alignment database produced in previous alignment steps. This mechanism allows to iterate track finding and alignment steps to minimize the risk that reconstructed tracks are compromised by telescope misalignment.

Track finding is a combinatorial problem which proceeds from seed tracks over candidate tracks to a final selection of reconstructed tracks in a telescope event. A seed consists of a pair of hits from the first two telescope layers which are used to trace out a straight line through the telescope with intersection at all other layers. Seeds are turned into candidates by adding further hits on other layers being close enough to the intersection predicted by the two seed hits. A natural measure for closeness are the local residuals or distances between the predicted intersection and the measured hit coordinates. In case the residuals on a layer are too large, no hit is added to the candidate. Otherwise, the hit with the smallest residuals is added to the candidate track. After processing all telescope layers, only candidate tracks with enough hits are kept for a final selection of reconstructed tracks among the candidates. The purpose of the final selection is to resolve possible overlaps between candidate tracks when the same hit is added twice. Secondly, the final track selection allows to impose quality cuts from a Kalman Filter fit to all hits found in the candidate.

The algorithm outlined above is rather slow as it requires to build candidate tracks for all possible combinations of seeded hits on the first two layers. The processing of a typical event may easily involve up to $\sim 1000$ seed tracks, most of them being random combinations of noise hits. A strategy is needed to allow a fast detection of bad seeds before attempting to enter the time consuming process of adding hits on other layers. Our method employs the strong angular collimation of particle beams at DESY and CERN: All real particle tracks are nearly parallel to the beam axis. In other words, a seed which forms a large angle with the beam axis is very likely to result from a wrong combination of seed hits and should 
be skipped right away. A useful angle cut is in the range of a few degrees relative to the beam axis and allows to remove the majority of bad seeds and substantially speeds up the track finding. Naturally, the efficiency of this method increases when the distance between the first two layers is decreased. For short layer distances, random combinations of hits are even more likely to form large angles with the beam axis.

Track finding is the first step in data reconstruction where precise information of the telescope geometry is needed. In order to compute a straight line connecting the two seed hits, their coordinates have to be transformed into global telescope coordinates first. Similarly, to compute the intersections of a straight line with other telescope layers, the position and orientation of the sensor planes must be known. Large uncertainties in the initial telescope geometry have a high potential to compromise the efficiency of track finding. Even for a good seed containing hits from a beam particle, other hits from this particle may not be found because a bad initial geometry makes the track finder to search at wrong places.

\subsubsection{Track fitting with Kalman Filters}

Track fitting uses the set of hits in a reconstructed track as a starting point. The goal is to estimate as accurately as possible a set of parameters describing the state of the particle as it traverses the silicon sensors in the telescope. Since the measured hit coordinates are stochastic and have statistical uncertainties, track fitting leads to statistical filtering procedures. In addition to the estimated values of the track states, the track fit also provides a measure of the uncertainty of these figures in terms of the covariance matrix of the state parameter vector.

Regarding the above mentioned main challenges in reconstruction, multiple scattering and tilted sensors, the situation is as follows: For a high momentum beam, the flight path of a charged particle through the EUDET beam telescope is very close to a $3 \mathrm{D}$ straight line and multiple scattering is frequently negligible. In this case, the only problem is to perform a least squares fit of a $3 \mathrm{D}$ straight line to the set of measured hits. If the sensor planes are parallel to each other, the fit is trivial. In a more general case, when the measured hits are bound on sensor planes with arbitrary rotations to each other, the problem becomes non linear and an iterative fit procedure must be applied. Especially for the validation studies presented in chapter 5 , the effect of the track incidence angle on the spatial resolution must be studied by placing the DEPFET sensor at various angular positions with respect to the beam direction ("tilt scan"). The $3 \mathrm{D}$ straight line fitter presented in Karimäki [64] handles this general case and can be used to estimate the intersection coordinates.

Karimäki's 3 D fitter works well for measurements with high momentum $(120 \mathrm{GeV})$ pions at the CERN SPS, but has severe limitations for the low momentum electrons at DESY due to multiple scattering. This is why the approach to track fitting followed in this thesis extends Karimäki's $3 \mathrm{D}$ fitter to a complete forward-backward Kalman Filter with an explicit model for multiple scattering. The application of Kalman Filters to track fitting was pioneered by Frühwirth [45] and has been developed into a standard technique for track fitting used in many experiments [65, 66]. The explicit formulation of a track model for the passage of a particle through the telescope with multiple scatterings is given in the appendix A The track model allows a precise estimation of the covariance matrix of track parameters taking into account measurement errors at sensors, errors from the propagation of track parameters between sensors, and (process) noise from multiple scattering at materials in the telescope. The process 
noise from multiple scattering is computed from the Highland model [28] which requires an explicit specification of the beam momentum and the distribution of all materials and their radiation length in the telescope.

In the following, we will discuss a number of important issues and adjustments to apply Kalman Filters for track fitting in beam telescopes. Firstly, we discuss how to get from local track parameters and their residuals to a measurement of the intrinsic spatial resolution of the devices under test. The method is formulated in a way that it can be applied to any sensor in the telescope. For the case of Mimosa26 sensors, the measurements confirm that an intrinsic spatial resolution of $3.5 \mu \mathrm{m}$ can be achieved for test beams at DESY. Furthermore, we discuss a new idea to parametrize the distribution of track parameters in the collimated particle beam at DESY. This collimated beam model can be used to initialize the track fit and helps to constrain telescope distortions like shearing and torsion with track data. Finally, we outline a new approach to estimate the radiation length $X / X_{0}$ at the device under test based on the estimation of scattering kinks with time reversed Kalman Filters.

\subsubsection{Local track parameters and residuals}

The Kalman Filter track fitter is designed to estimate the state of the particle as it enters the silicon sensor before multiple scattering takes place. The track state is parametrized relative to the measurement plane $w_{k}=0$ of the $k$ th telescope layer by a four dimensional parameter vector

$$
\lambda_{k}=\left(t_{u}, t_{v}, u, v\right)^{T}
$$

where $t_{u}=d u / d w$ and $t_{v}=d v / d w$ are the local directions tangents (incident angles) and $u, v$ are the local intersection coordinates. The statistical uncertainty of the parameter values is described by a $4 \times 4$ parameter covariance matrix $C_{k}$. The formulation of the track model with local state variables offers a number of advantages for a beam telescope. In the case of tilt scans, the local incident angles are closely related to a number of important observables on the tilted sensor like the cluster size, the average energy loss and the spatial resolution. Especially, the estimated length of the ionizing path of a charged particle in a silicon sensor with thickness $T$ can be computed as

$$
l=T \sqrt{1+t_{u}^{2}+t_{v}^{2}} .
$$

and should be directly proportional to the average energy loss of the particle. For a study of the intrinsic spatial resolution at layer $k$, the key variables are the predicted residuals

$$
r_{k}=m_{k}-H \lambda_{k}, \quad H=\left(\begin{array}{cccc}
0 & 0 & 1 & 0 \\
0 & 0 & 0 & 1
\end{array}\right)
$$

where $m_{k}=\left(u_{m}, v_{m}\right)^{T}$ denotes the local hit coordinates and $H$ is a measurement projection matrix. As described in the appendix A.2.3 in more detail, the estimation of the local track state $\lambda_{k}$ is designed in a way to use all hits $m_{j}$ but excludes the hit at layer $k$ itself. This choice ensures that the track parameters $\lambda_{k}$ are statistically uncorrelated to the hit coordinates $m_{k}$. The residual covariance $R_{k}$ is the sum of the measurement covariance $V_{k}$ and the telescope pointing covariance $H C_{k} H^{T}$.

$$
R_{k}=H C_{k} H^{T}+V_{k}
$$


Measurements with the EUDET beam telescope allow to measure the residual covariance $R_{k}$ from a sufficiently large sample of reconstructed beam particles hitting the device under test. For the case of perpendicular incidence of high momentum particles at CERN, the telescope pointing covariance is frequently small compared to the hit covariance and can be neglected, $V_{k} \cong R_{k}$. However for increasing tilts of the sensor under study, the hit covariance shrinks while the telescope covariance generally grows. In the worst case, both covariance matrices are of similar size and an explicit subtraction of the telescope covariance is necessary, $V_{k}=R_{k}-H C_{k} H^{T}$. The accuracy of the result depends on the correct estimation of the telescope covariance by the track fitter. Especially, the straight line fitter generally ignores noise from multiple scattering and significantly underestimates the telescope covariance for low momentum particles. In appendix A.3, detailed simulation experiments are performed to demonstrate that the track fitter developed here allows a correct assessment of the telescope covariance.

The method described above can be used to measure the spatial resolution of Mimosa26 modules from track residuals. In a previous study on test beam measurements with the EUDET telescope [41], it was shown that the correlations between the residuals along the $u$ and $v$ coordinates of the Mimosa26 sensors are very small. If we neglect these small correlations, we obtain the following simplified formula for the spatial resolution at layer $k$ :

$$
\sigma_{u, k}=\sqrt{\sigma_{r, k}^{2}+\sigma_{t, k}^{2}}
$$

Here, the variables $\sigma_{r, k}$ and $\sigma_{t, k}$ are the square roots of the respective diagonal entries in the covariance matrices $R_{k}$ and $H C_{k} H^{T}$. The variables $\sigma_{r, k}$ and $\sigma_{t, k}$ are called the residual width and the telescope pointing resolution at layer $k$. In Fig. 4.5 , the distribution of the residuals in the $u$ direction is shown for all six Mimosa26 modules in a $3.75 \mathrm{GeV}$ electron beam at DESY 3 , The residuals are centered around zero. The curves fitted to the histograms are Gaussian and the fitted mean and standard deviations are given at all layers. The residual width $\sigma_{r, k}$ is estimated as the standard deviation of the Gaussian fit and strongly depends on the $z$ position of the module in the telescope as can be seen in Fig.4.6. However, after subtracting the telescope resolution $\sigma_{t, k}$ from the residual width according to Eq. 4.7, the measured spatial resolutions are rather flat as a function of $z$ and lie closely around the expected value of $3.5 \mu \mathrm{m}$. The remaining deviations are mostly due to systematic uncertainties on the telescope resolution at the level of $\sim 5 \%$. The result demonstrates that the track fitter developed in this thesis allows an accurate estimation of the telescope resolution even in the case of strong multiple scattering.

\subsubsection{A model for track parameters in collimated particle beams}

The particles tracked through the beam telescope originate from a highly collimated particle beam. Our idea is to utilize the beam collimation for the alignment of the telescope from track data. More precisely, we outline the approach to align the $z$ axis of the global coordinate system with the direction of the particle beam and to explicitly parametrize the first and second moments of the distribution of track parameters of beam particles. These moments can be used as an a priori statistics for the track fitter. In addition, the modeling of moments gives new constraints for the alignment of the beam telescope and allows to suppress the telescope distortions known as shearing and torsion.

\footnotetext{
${ }^{3}$ The telescope setup with all sensor distances is shown in Fig. 4.2
} 

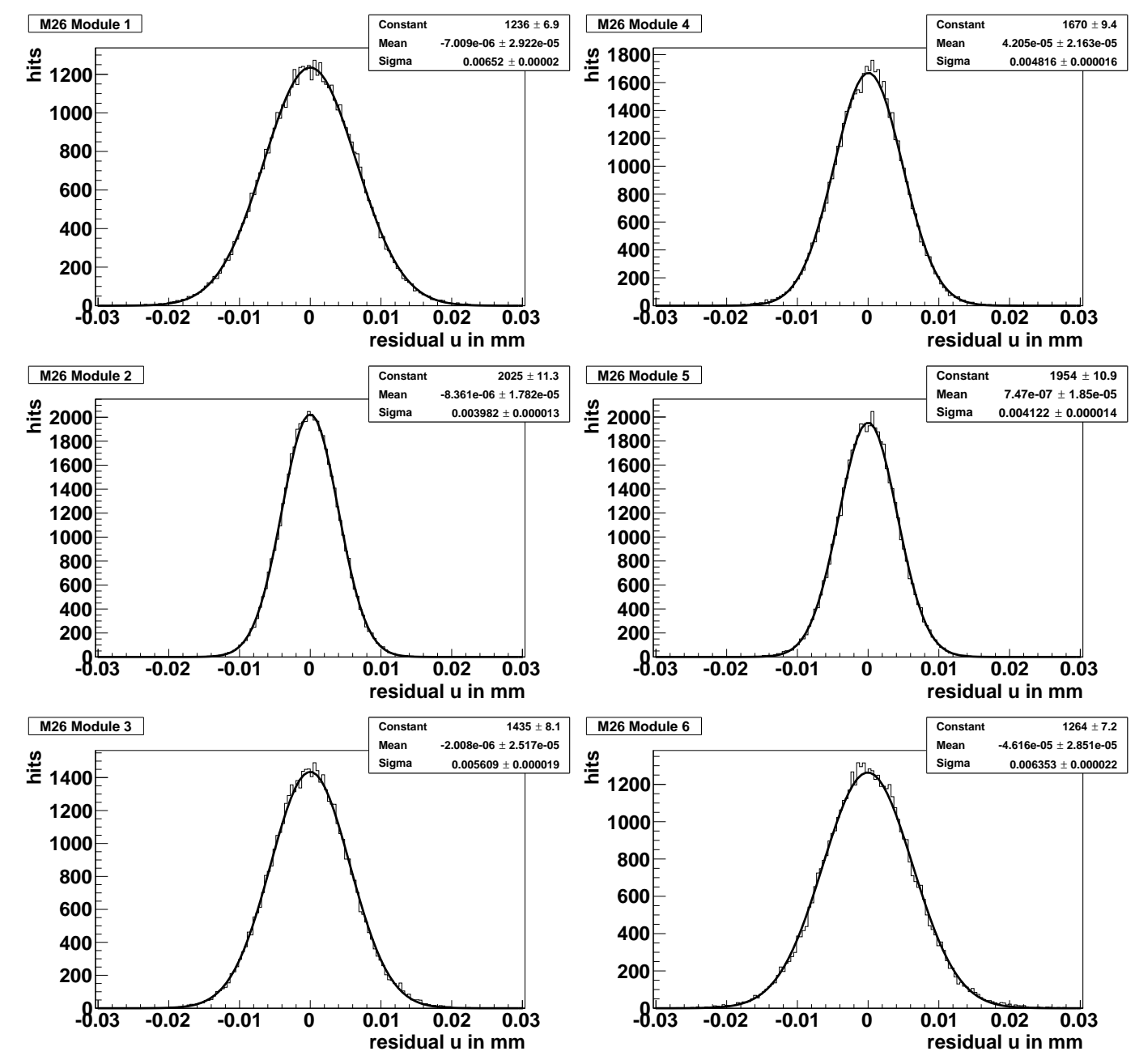

Figure 4.5.: Residuals in the local $u$ direction for all Mimosa26 modules in the EUDET telescope. The modules are numbered along the beam line with No. 1 at the first position in the telescope. A Gaussian is fitted to all residuals and the fitted mean and sigma values are shown. The residuals are centered around zero which shows that the alignment was successful. The width of the residuals depends on the position of the module in the telescope. 
4. The reconstruction of beam telescope data: methods, steps and calibration

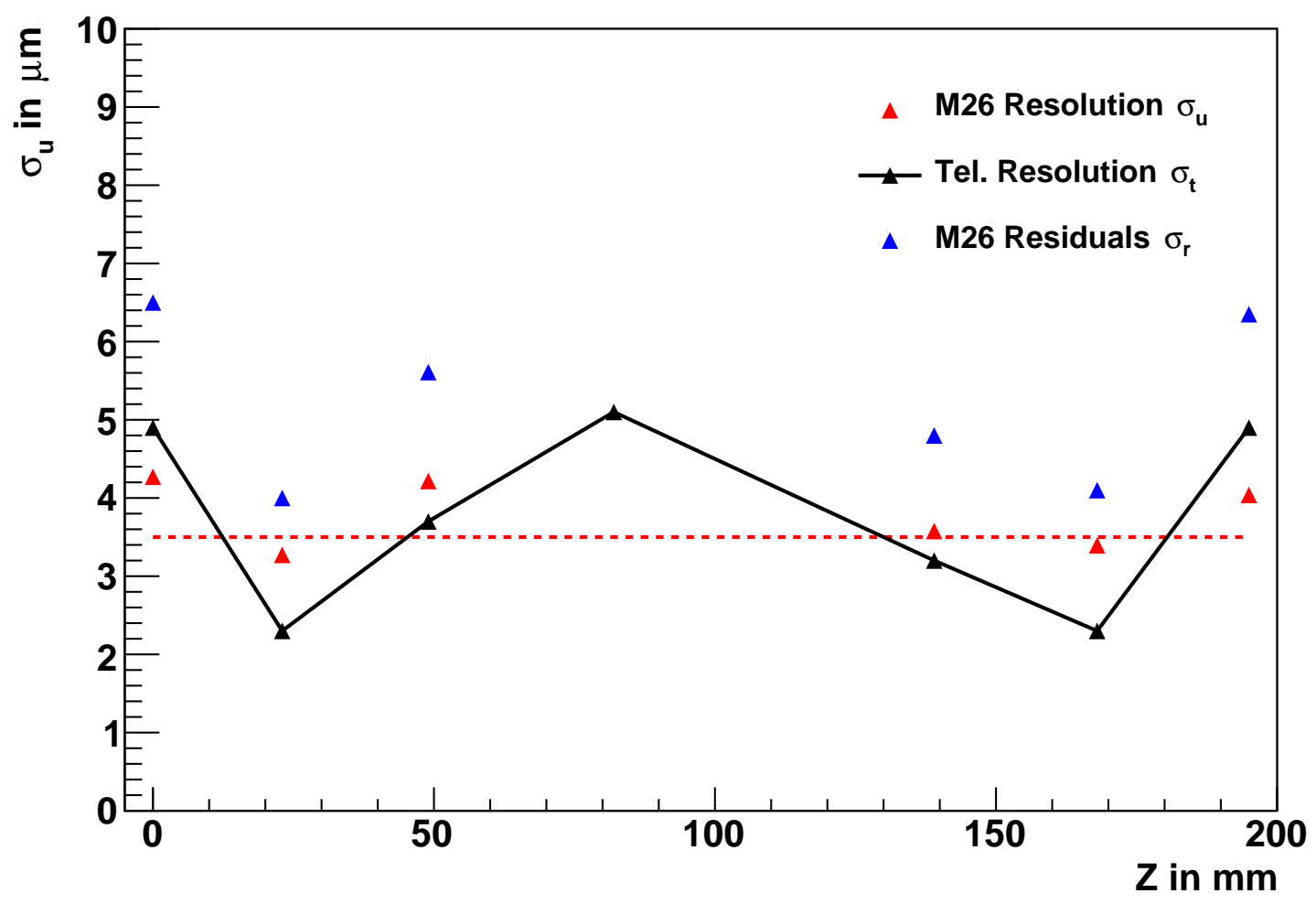

Figure 4.6.: The measured spatial resolution, $\sigma_{u}$, as a function of the telescope $z$ coordinate. The spatial resolution is computed by subtracting the telescope resolution from the measured residual width. As can be expected, the measured spatial resolution of all Mimosa26 modules is almost independent from the $z$ position in the telescope and lies around the expected value of $3.5 \mu \mathrm{m}$. The telescope resolution is $5 \mu \mathrm{m}$ at the position of the DEPFET module between the two telescope arms. 
Without loss of generality ${ }^{4}$, we consider the global $z=0$ plane as a further telescope layer placed before all other layers which follow at positions $0<z_{1}<\cdots<z_{n}$ along the $z$ axis. At this telescope layer, the track parameters are given relative to the $z=0$ plane

$$
\lambda_{0}=\left(\begin{array}{c}
t_{x} \\
t_{y} \\
x \\
y
\end{array}\right)
$$

The approach to align the global $z$ axis of the telescope with the direction of the particle beam can now be formulated more precisely as an equality constraint on the mean value of track parameters at the $z=0$ plane.

$$
<\lambda_{0}>=\left(\begin{array}{l}
0 \\
0 \\
0 \\
0
\end{array}\right)
$$

In other words, beam particles intersect the $z=0$ plane around the origin of the global coordinate system at $x=y=0$ and the average flight direction is parallel to the $z$ axis. To model the covariance matrix of track parameters, we assume that the track parameters decouple in the $x-z$ and $y-z$ plane. In this case, the most general parameter covariance matrix can be written as

$$
C_{0}=\left(\begin{array}{cccc}
\sigma_{t_{x}, t_{x}}^{2} & 0 & \sigma_{t_{x}, x}^{2} & 0 \\
0 & \sigma_{t_{y}, t_{y}}^{2} & 0 & \sigma_{t_{y}, y}^{2} \\
\sigma_{t_{y}, y}^{2} & 0 & \sigma_{x, x}^{2} & 0 \\
0 & \sigma_{t_{y}, y}^{2} & 0 & \sigma_{y, y}^{2}
\end{array}\right)
$$

The covariance matrix in Eq. 4.10 assumes an elliptical beam spot having a spot size of $\sigma_{x x}$ and $\sigma_{y y}$ in the horizontal direction $(x)$ and the vertical direction $(y)$. The angular spread of beam particles around the $z$ axis is described by the beam divergence $\sigma_{t_{x}, t_{x}}$ for the $x-z$ plane and $\sigma_{t_{y}, t_{y}}$ for the $y-z$ plane. The beam particle spread out along the beam direction due to multiple scattering and we can expect a positive correlation between the track intersection coordinates and the track incident angles. In the simplest case, the situation can be described by two linear beam correlation coefficients.

$$
\begin{aligned}
& r_{x}=\frac{\sigma_{t_{x}, x}^{2}}{\sigma_{x, x} \sigma_{t_{x}, x}} \geq 0 \\
& r_{y}=\frac{\sigma_{t_{y}, y}^{2}}{\sigma_{y, y} \sigma_{t_{y}, y}} \geq 0
\end{aligned}
$$

In Fig. 4.7 and Fig. 4.8 we show the distribution of reconstructed track intersection coordinates, incident angles and their correlations for a $3.75 \mathrm{GeV}$ electron run at DESY. The reconstruction of track parameters is performed in a fully aligned EUDET telescope operated with a single large scintillator in front. Within the telescope aperture, the measured beam spot size is $10 \pm 1 \mathrm{~mm}$ in the horizontal and

\footnotetext{
${ }^{4}$ In general, there is some degree of freedom regarding the choice of a global coordinate system. In particular, the reconstruction results do not depend on global shift and rotations of both the telescope sensors and the particle source.
} 

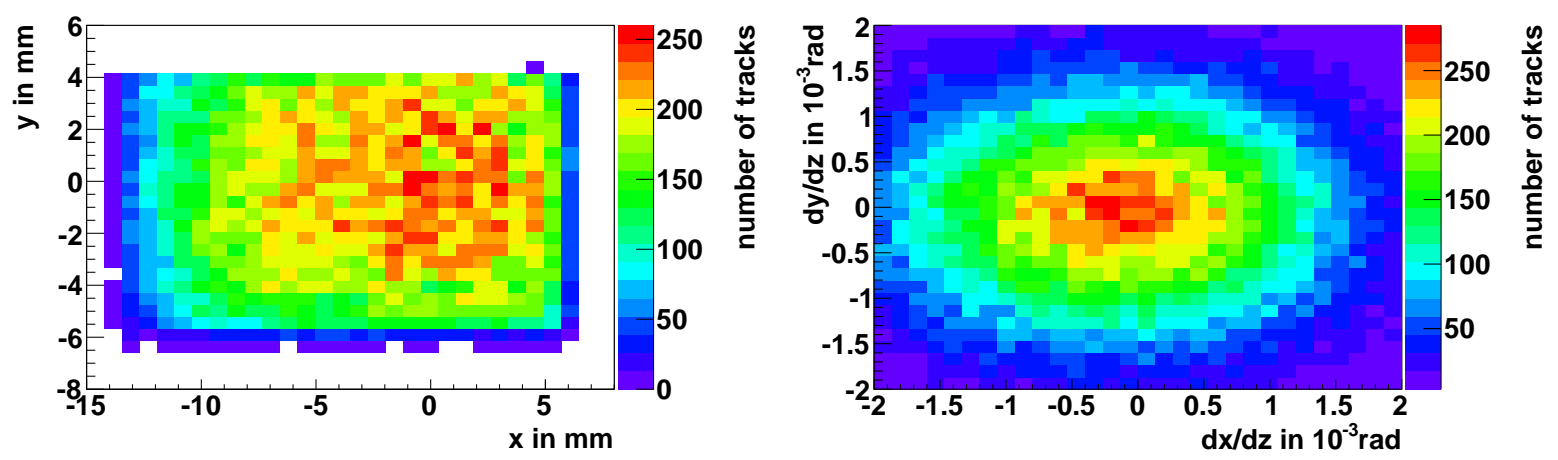

Figure 4.7.: Left: The distribution of track intersection coordinates at the $z=0$ plane for all reconstructed particle tracks with hits on all Mimosa26 sensors. The range of track intersections is limited to a rectangular field of $10 \times 20 \mathrm{~mm}^{2}$ defined by the active area of the Mimosa26 sensors. The central part of the beam spot is visible at the origin of the global coordinate system. Right: The distribution of track incident angles $d x / d z$ and $d y / d z$ at the $z=0$ plane for reconstructed tracks. The beam axis is aligned to the $z$ axis during the telescope alignment.

$6 \pm 1 \mathrm{~mm}$ in the vertical direction. Similarly, the measured beam divergence is $1.1 \times 10^{-3} \mathrm{rad}$ in the $x-z$ plane and $0.9 \times 10^{-3}$ rad in the $y-z$ plane. As can be seen in Fig. 4.8 , the track parameters show a linear correlation between track incident angles and intersection coordinates. The beam correlation coefficients are estimated to $0.7 \pm 0.2$.

This model can be employed in two ways. Firstly, the intersection of the beam axis with the sensor planes gives a reference flight path for the linearization of the track model. The strategy for the linearization of Kalman Filters for track fitting is described in [66]. Secondly, the collimation of beam particles is a property of the beam line and can be regarded as an independent further information about the beam particles. The moments $\left\langle\lambda_{0}>\right.$ and $C_{0}$ are usable as a priori statistics for the track fitter in addition to the reconstructed hits. These properties of the particle beam will be used for the alignment of the telescope described in section 4.5 .4 .

\subsubsection{Multiple scattering and estimation of the radiation length}

The Kalman Filter based track fitter developed in this thesis has already been used by Stolzenberg [67] and Wieduwilt [68] for a position resolved measurement of the radiation length $X / X_{0}$ of DEPFET pixel modules in the EUDET beam telescope. Our new approach to material estimation is based on the explicit reconstruction of the scattering kinks of a particle at the device under test. The main idea is to run two Kalman Filters in opposite directions on the reconstructed hits. A forward filter is run in the direction of the particle to estimate the track parameters before scattering at the device under test. Secondly, a time reversed filter is run in the opposite direction to estimate the local track parameters after scattering at the device under test. The difference between the incident angles before and after scattering is a measurement of the scattering kink angle, or shortly kink, at the device under test. The precise definition of the two Kalman Filters used for material estimation and the computation of the covariance of the measured scattering kinks is given in appendix A.2.2. The width of the distribution of the scattering kink at a given position on the device under test can be related to the local radiation 

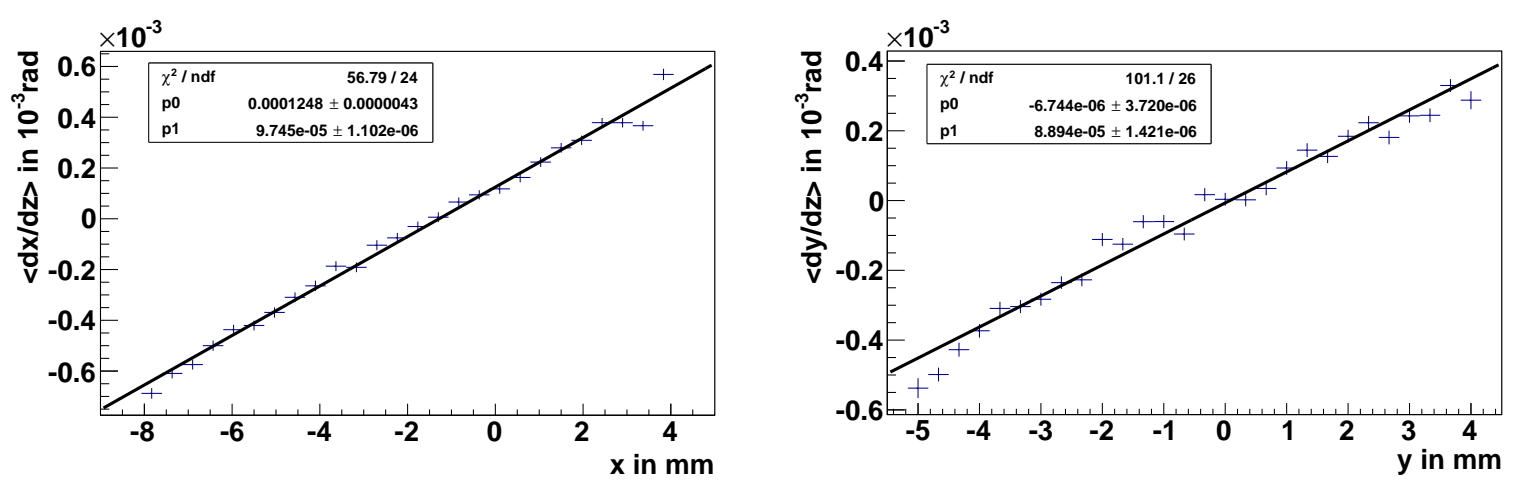

Figure 4.8.: Left: Profile of the track incident angles $d x / d z$ and the $x$ intersection coordinate at the $z=0$ plane. The linear correlation between the intersection coordinates and the incident angle is clearly visible. The correlation coefficient $r_{x}$ is extracted from a linear fit. Right: Profile of the track incident angles $d y / d z$ and the $y$ intersection coordinate at the $z=0$ plane. The linear correlation between the intersection coordinates and the incident angle is clearly visible. The correlation coefficients $r_{x}$ and $r_{y}$ are proportional to the slope of the line fit.

length; for details see [68]. To illustrate the potential of the new approach, a map of the radiation length of a DEPFET pixel module obtained from low momentum electrons is shown in Fig.4.9.

\subsection{The telescope alignment (Step 3)}

Track fitting can attain its ultimate precision only if the positions and orientations of the sensors are known with high precision. Alignment methods are used for the precise determination of the position and orientation of the sensors in the telescope. Telescope alignment is a highly nontrivial task that can only be solved by using information from charged particles that cross several sensors along their trajectory through the telescope. Alignment is therefore intimately connected to track fitting.

The scope of this section on telescope alignment is the following: Based on a brief overview of the goals of telescope alignment and its problems, we discuss the parametrization of sensor position errors, followed by an attempt to quantify the errors in the initial geometry data base. Finally, we present an optimized approach towards a better telescope alignment. A new method for robust pre-alignment with hits is presented to compute the correction of the sensor $x$ and $y$ position relative to the beam axis. The method utilizes the collimated beam model and can be used to pre-align the telescope for a more efficient and robust track finding. This pre-alignment is followed by an alignment with tracks using the Kalman Alignment Algorithm with Annealing, as presented in Frühwirth [18].

\subsubsection{Goals and problems of telescope alignment}

Misalignment can be seen as the difference between the assumed ideal position of a given sensor with respect to its true position. In the presence of misalignment, the local track parameters estimated in track fitting are biased. The goal of telescope alignment is to accurately determine the sensor positions so that parameter biases become negligible compared to the statistical uncertainty of track parameter given by the track parameter covariance matrix. In Tab. 4.3, we summarize the statistical resolutions 


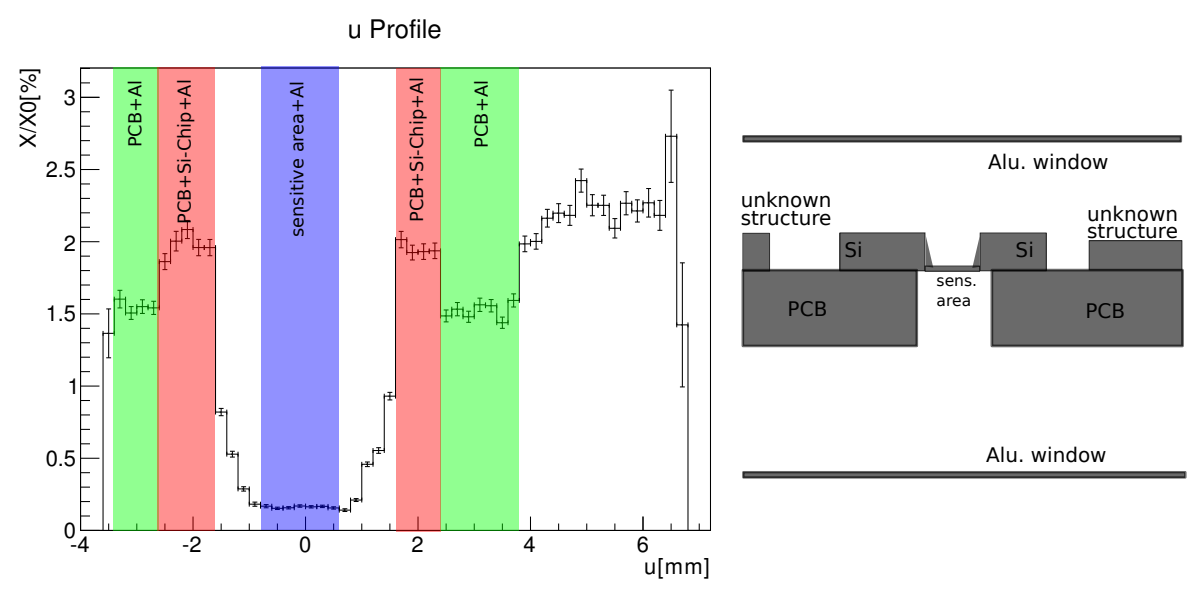

Figure 4.9.: Position resolved map of the radiation length of a DEPFET prototype module measured at a $3.75 \mathrm{GeV}$ electron beam with the EUDET telescope at DESY [67]. The sensitive silicon is thinned from $420 \mu \mathrm{m}$ to $50 \mu \mathrm{m}$ using deep anisotropic etching. The thickness of an aluminum window is roughly $80 \mu \mathrm{m}$. The measured radiation length the radiation length of all materials struck by the particle.

\begin{tabular}{|c|c|c|c|}
\hline$\delta u$ & $\delta v$ & $\delta t_{u}$ & $\delta t_{v}$ \\
\hline \hline$\sim 2 \mu \mathrm{m}$ & $\sim 2 \mu \mathrm{m}$ & $\sim 10^{-4} \mathrm{rad}$ & $\sim 10^{-4} \mathrm{rad}$ \\
\hline
\end{tabular}

Table 4.3.: Typical values for the track parameter resolution at a single device under test sensor in the center of the EUDET telescope at DESY. The given values refer to an optimized telescope geometry with a spacing of $30 \mathrm{~mm}$ between all sensor planes in a $3 \mathrm{GeV}$ electron beam. In the ideal case, track parameter biases from telescope misalignment should be smaller than these statistical errors.

for track parameters which can be reached at the center of the EUDET telescope at DESY. In the ideal case, the biases of extrapolated intersections should be well below one micrometer and biases of incident angles should be below $10^{-4}$ radians. These errors are at least one order of magnitude smaller than the placement accuracy achieved during telescope installation.

The established method to determine the true sensor positions is to use measurements from particles traversing the telescope and minimizing the residuals of an appropriate track model [69]. The problems of this alignment with tracks and some solutions which are developed in this thesis are the following:

- For the case of low momentum beams at DESY, an appropriate track model is supposed to take care of multiple scattering and telescopes with tilted sensors. By applying the Kalman Alignment Algorithm, it is possible to re-use the Kalman Filter track fitter presented in the last section.

- The size of the data sample needed is in the order $\sim 10 \mathrm{k}$ tracks crossing all telescope sensors including the devices under test. In order to find these tracks, the telescope must be pre-aligned. To get started, we use a robust pre-alignment with beam constrained tracks, i.e. tracks going through a single hit on the first layer and flying parallel to the beam axis.

- Certain global distortions (shearing, torsion, stretching) of the telescope geometry leave track residuals invariant and cannot be corrected by track based methods. These so called weak modes give a systematic bias to track incident angles which is far larger than the statistical error from the 
track fitter. Using the collimated beam model in the track fitter helps to constrain shearing and torsion. However, stretching must be constrained from mechanical measurements.

- The small divergence of the particle beam limits the sensitivity of track based alignment to certain alignment parameters. The sensitivity is smallest for the correction of tilts $\alpha$ and $\beta$ around the telescope $x$ and $y$ axes when the sensor plane is nearly perpendicular to the beam direction. For sensors tilted to the beam axis by more than approximately one degree, the track data provides enough sensitivity for a successful correction. Otherwise these tilts should be neglected.

\subsubsection{The parametrization of telescope misalignment}

To parametrize telescope misalignment, we follow the approach presented by Karimäki [69] where a silicon sensor is considered to be a rigid body and its position in space is implicitly defined by the transformation from local sensor to global telescope coordinates.

The nominal position of the $i$ th sensor is specified by a displacement vector $\vec{r}_{i}$ and a rotation matrix $R_{i}$ as discussed in section 3.2.2. However, the true position of the sensor in space is given by the displacement vector $\vec{r}_{i}^{\star}$ and a rotation matrix $R_{i}^{\star}$. The relation between the nominal and true sensor positions is given by

$$
\begin{aligned}
R_{i}^{\star} & =\Delta R_{i}^{\star} R_{i} \\
\vec{r}_{i}^{\star} & =\vec{r}_{i}+\Delta \vec{r}_{i}^{\star}
\end{aligned}
$$

where vector $\Delta \vec{r}_{i}^{*}=\left(\Delta x_{i}, \Delta y_{i}, \Delta z_{i}\right)$ shifts the sensor center from the nominal to its true position and the rotation matrix $\Delta R_{i}^{\star}$ tilts the nominal sensor plane. In the approximation of small tilts, we can write the rotation matrix in the following way 5 .

$$
\Delta R_{i}^{\star}=\left(\begin{array}{ccc}
1 & \Delta \gamma_{i} & \Delta \beta_{i} \\
-\Delta \gamma_{i} & 1 & \Delta \alpha_{i} \\
-\Delta \beta_{i} & -\Delta \alpha_{i} & 1
\end{array}\right)
$$

Here, $\Delta \alpha_{i}, \Delta \beta_{i}$ and $\Delta \gamma_{i}$ are small tilts around the nominal $u, v, w$ sensor axes. Thus, the misalignment of a single sensor is parametrized by a six dimensional vector $a_{i}^{\star}=\left(\Delta x_{i}, \Delta y_{i}, \Delta z_{i}, \Delta \alpha_{i}, \Delta \beta_{i}, \Delta \gamma_{i}\right)$ of alignment parameters. The shifts are given in units of $\mathrm{mm}$ and the tilts in units of radians. The task of alignment is to produce an estimate $a_{i}$ of the true alignment parameters $a_{i}^{\star}$ for all sensors in the telescope. The estimated alignment parameters can be used to update the sensor position according to:

$$
\begin{aligned}
R_{i}^{u p} & =\Delta R_{i} R_{i} \\
\vec{r}_{i}^{u p} & =\vec{r}_{i}+\Delta \vec{r}_{i}
\end{aligned}
$$

The updated sensor positions can be used to overwrite the geometry data base. Then, the updated geometry data base gives a new starting point for further telescope alignment steps. In general, telescope

\footnotetext{
${ }^{5}$ If tilt angles $x$ are small enough $\left(\sim 10^{-3} \mathrm{rad}\right)$, we use the first order linearization of the trigonometric functions: $\sin x=x$
} and $\cos x=1$ 
alignment should be repeated until all alignment parameters have converged to zero.

So far, the telescope misalignment was described in a local way sensor by sensor. It is possible to use these local degrees of freedom in a correlated way to create distortions of the whole telescope. For a tracking telescope, we are concerned with those global distortions which displace sensors in such a way that hits from high momentum particles would lie on a straight line before and after the alignment correction is applied. For beam telescopes, there are exactly four such global distortions which can be written as $z$ dependent shifts of the alignment parameters.

$$
\begin{aligned}
\Delta x_{i} & =a_{x}+b_{x} \cdot z_{i} \\
\Delta y_{i} & =a_{y}+b_{y} \cdot z_{i} \\
\Delta z_{i} & =a_{z}+b_{z} \cdot z_{i} \\
\Delta \gamma_{i} & =a_{\gamma}+b_{\gamma} \cdot z_{i}
\end{aligned}
$$

The first two equations describe a shearing of the telescope in the $x$ and $y$ direction while the third equation models a rescaling of the sensor distances along the beam line called stretching. Finally, the fourth equation describes a $z$ dependent rotation of the sensors around the $z$ axis called torsion. In the context of alignment, these global distortions of the telescope geometry are also known as weak modes.

For track based alignment, weak modes are very difficult to detect from track residuals as hits in the distorted telescope geometry still line up along a straight line. However, weak modes can lead to significant biases in fitted track parameters. As can be seen from Eq.4.14 and Eq.4.15, the fitted track intersections with the $z=0$ plane are biased by the shearing offsets $a_{x}, a_{y}$ while the fitted track incident angles are biased by the shearing amplitudes $b_{x}, b_{y}$. Large biases on the incident angles are especially problematic for the precise estimation of the material traversed by a particle in the telescope and angle resolved measurements of the spatial resolution for tilted devices under test. In the case of a telescope torsion, a straight track going parallel to the $z$ axis will be reconstructed as an helical track in distorted telescope geometry. Depending on the value of torsion amplitude $b_{\gamma}$ in Eq.4.17, the telescope measures hits only along a small segment of this helix. In this case, torsion leads to a position dependent bias on the fitted track directions as can be seen in Fig. 4.12 .

\subsubsection{Survey measurements and initial telescope misalignment}

In the previous section, we have described telescope misalignment both on a local and a global level. Now, we need to quantify how much misalignment should be anticipated in a real experiment. Essentially, our task is to come up with a realistic table of initial errors for both local and global alignment parameters.

The initial telescope geometry assumes that the six Mimosa26 reference sensors are placed perpendicular to the beam line. The distances $z_{i}$ between sensors are measured in mechanical surveys and are optimized to yield a high telescope pointing resolution at the device under test. The initial geometry parameters for the Mimosa26 reference sensors are 


\begin{tabular}{|c|c|c|c|c|c|}
\hline$\Delta x[\mathrm{~mm}]$ & $\Delta y[\mathrm{~mm}]$ & $\Delta z[\mathrm{~mm}]$ & $\Delta \alpha[\mathrm{mrad}]$ & $\Delta \beta[\mathrm{mrad}]$ & $\Delta \gamma[\mathrm{mrad}]$ \\
\hline \hline$\sim 1-5$ & $\sim 1-5$ & $\sim 1-5$ & $\sim 20$ & $\sim 20$ & $\sim 20$ \\
\hline
\end{tabular}

Table 4.4.: Conservative estimation of the RMS errors of the initial telescope geometry after mechanical installation of sensor boxes on the telescope support table.

$$
r_{k}=\left(\begin{array}{c}
0 \\
0 \\
z_{k}
\end{array}\right) \quad R_{k}=D_{k}=\left(\begin{array}{ccc}
d_{1} & d_{2} & 0 \\
d_{3} & d_{4} & 0 \\
0 & 0 & d_{5}
\end{array}\right)
$$

where the index $k$ enumerates all sensors along the beam line and $D_{k}$ is the discrete rotation matrix discussed previously (see section 3.2.2). In particular, the initial geometry assumes that the initial values of the variables $\Delta x, \Delta y, \Delta \alpha, \Delta \beta, \Delta \gamma$ are set to zero for all reference sensors. For tilt scans of the devices under test, initial values for the tilts $\alpha$ and $\beta$ are determined from the settings of the rotation stage. The telescope misalignment in the real telescope will be referenced to this initial geometry.

Precise mechanical survey measurements are difficult due to the fact that sensors are not easily accessible. Typically, silicon sensors are glued to a printed circuit board (PCB) which in turn is screwed to a light shielding box. The detector box is either directly screwed to the telescope support table or installed on an $X Y$ stage between the telescope arms. The track system of the EUDET support table provides a precise positioning of the Mimosa26 sensors relative to each other. However, the device under test sensors are installed without a track system and their initial positions can be shifted by several millimeters relative to the Mimosa26 sensors. In practice, mechanical surveys are limited to a rough measurement of the distances between the detector boxes along the beam direction, see for example Fig. 4.2 . For the remaining degrees of freedom, we can only give a conservative estimation of the error on the initial sensor position, see Tab. 4.4 .

The maximum possible shearing in the initial telescope geometry can be estimated under the assumption that the beam axis intersects the active sensor area $W \times H$ of the first and the last sensor in the telescope. In this case, the relevant geometrical parameters are the distance $L$ between the first and the last Mimosa26 sensor as well as the width $W$ and height $H$ of Mimosa26 sensors. Tab. 4.5 shows that the shearing amplitudes $b_{x}$ and $b_{y}$ defined in Eq.4.14 and 4.15 can be as large as $100 \mathrm{mrad}$ in the worst case. In other words, without further alignment corrections to suppress telescope shearing, the estimated track incident angles can be biased by up to $100 \mathrm{mrad}$. The numbers given in Tab. 4.5 can be interpreted as precision requirements for the mechanical positioning of the telescope support table relative to the particle beam. For proper positioning of the EUDET telescope in the particle beam, the $x$ and $y$ position of the telescope table relative to the beam axis should be controlled to the level of mm while rotations of the telescope table should be controlled to the level of a few milliradians.

A similar argument can be used to derive worst case values for telescope stretching and torsion relative to the initial geometry, see Tab.4.6. For stretching, the mechanical measurement of the total telescope length is accurate to $\sim 1 / 200$ and typically no further correction from track data to the $z$ position of the first and last sensor is needed. However, the $z$ positions of inner sensors should be fine-tuned in the alignment with tracks. The required accuracy on $z$ positions is in the order of $\sim 100 \mu \mathrm{m}$ and cannot be reached from mechanical measurements alone. The situation is a bit different for telescope torsion. Torsion has a very characteristic fingerprint on the beam profile (see Fig. 4.12) that can be used to 


\begin{tabular}{|c|c|c|c|}
\hline$a_{x}[\mathrm{~mm}]$ & $a_{y}[\mathrm{~mm}]$ & $b_{x}[\mathrm{rad}]$ & $b_{y}[\mathrm{rad}]$ \\
\hline \hline$H=10.6$ & $W=21.2$ & $H / L=0.05$ & $W / L=0.1$ \\
\hline
\end{tabular}

Table 4.5.: Estimation of maximum values for telescope shearing. The four shearing parameters $a_{x, y}$ and $b_{x, y}$ are defined in Eq. 4.14 and 4.15 and values are relative to the initial telescope geometry. The width $W$ and height $H$ of the Mimosa26 sensors are used. The length $L$ of the telescope is assumed to be $200 \mathrm{~mm}$. A length of $200 \mathrm{~mm}$ is a typical value for test beam measurements presented in this thesis.

\begin{tabular}{|c|c|c|c|}
\hline$a_{z}[\mathrm{~mm}]$ & $a_{\gamma}[\mathrm{mrad}]$ & $b_{z}[\mathrm{mrad}]$ & $b_{\gamma}[\mathrm{mrad}]$ \\
\hline \hline$\Delta z \sim 1-5$ & $\Delta \gamma \sim 20$ & $\Delta z / L \sim 5-25$ & $\Delta \gamma / L \sim 0.1$ \\
\hline
\end{tabular}

Table 4.6.: Estimation of maximum values for telescope stretching and torsion relative to the initial telescope geometry. The offsets and amplitudes for torsion and stretching are estimated from the sensor level misalignment $\Delta z$ and $\Delta \gamma$ of the first and the last sensors in the telescope. The total length of the telescope is $L$.

suppress telescope torsion.

\subsubsection{A new approach to better telescope alignment}

In practice, we use a combination of different algorithms to achieve a good alignment of the telescope. The next section provides an overview of the various algorithms. Next, we outline the global alignment strategy and give some tips and tricks that proved to be useful for telescope alignment. Finally, we study the performance of telescope alignment procedure on real data.

\subsubsection{Pre-Alignment: Beam spot correction and hit correlations}

Large initial errors of sensor positions degrade the efficiency of track finding and lead to large biases in estimated track parameters. As discussed in the last section, especially the position of a tilted device under test is poorly known and poses two problems:

- To align the device under test with tracks, we need to have enough tracks with hits on the device under test. However, the track finder is hampered by the badly known position of the device under test.

- A device under test may be small and noisy compared to the Mimosa26 reference sensors. In order to add the right hit on the device under test in the track finder, we need to know its position well.

The solution to these problems is a robust pre-alignment of the $x$ and $y$ position of sensors relative to the beam direction. At first, we compute the correction of the $x$ and $y$ position of the first sensor based on the position of the beam spot on its sensitive area. In a second step, the $x$ and $y$ positions of all other sensors are corrected using hit correlations between sensors.

\section{The beam spot correction}

For a telescope that is well positioned in the beam, the center of the beam spot is visible as a maximum in the density of hits on the local $u-v$ plane of the sensor. In Fig. 4.10, we show a map of the hit density of 
a Mimosa26 plane in a test beam at DESY computed from $100 \mathrm{k}$ events in a run with $3.75 \mathrm{GeV}$ electrons. Around the center of the beam spot, the hit density in the $u-v$ plane can be modeled by a $2 D$ Gaussian probability density.

$$
\rho(u, v)= \begin{cases}\frac{1}{N} \exp \left[\left(u-u_{b}\right)^{2} / \sigma_{b, u}^{2}+\left(v-v_{b}\right)^{2} / \sigma_{b, v}^{2}\right] & , \text { inside active area } \\ 0 & , \text { outside active area }\end{cases}
$$

Here, the variables $u_{b}, v_{b}$ are the local intersection coordinates of the beam axis on the sensor plane and $\sigma_{b, u}$ and $\sigma_{b, v}$ are the sizes of the beam spot and $N$ is a normalization factor. The fitted size of the beam spot is $7 \pm 1 \mathrm{~mm}$ in $u$ and $10 \pm 1 \mathrm{~mm}$ in the $v$ direction. The central area of the beam spot is $7 \times 10 \mathrm{~mm}^{2}$ while the active area of the Mimosa26 sensor is $10 \times 20 \mathrm{~mm}^{2}$. The positioning of the beam spot on the active sensor area is challenging but feasible by a careful positioning of the telescope support table relative to the particle beam.

We can use the fitted values for $u_{b}$ and $v_{b}$ to correct the initial $x$ and $y$ positions of the sensor relative to the beam axis. In the initial geometry, the estimated beam axis intersection is at

$$
\overrightarrow{q_{b}}=\left(\begin{array}{c}
u_{b} \\
v_{b} \\
0
\end{array}\right)
$$

while the global coordinates of this intersection point are

$$
\vec{r}_{b}=\left(\begin{array}{c}
x_{b} \\
y_{b} \\
z_{0}
\end{array}\right)=R_{0}^{T} \vec{q}_{b}+\vec{r}_{0}
$$

Here $\vec{r}_{0}$ and $R_{0}$ denote the initial sensor shift and rotation matrix. In other words, the real position of the sensor center is shifted from the beam axis by a distance of $x_{b}$ and $y_{b}$ in global coordinates. In order to align the beam axis with the telescope $z$ axis, we have to shift the sensor center by $\Delta x=x_{b}$ and $\Delta y=y_{b}$ away from the telescope $z$ axis. The precision of the sensor shifts $\Delta x$ and $\Delta y$ is typically in the order of $\sim 100 \mu \mathrm{m}$. The correction of the sensor position relative to the beam axis is an important first step to suppress shearing in the initial telescope geometry.

\section{Alignment with beam constrained tracks}

After the beam spot correction, we can assume that the $x$ and $y$ position of the first sensor in the telescope is well controlled and will be fixed to provide a reference point in the remaining alignment steps. In order to correct the $x$ and $y$ positions of the other sensors in the telescope, we will present an extension of the hit correlation method presented in Behr [41]. The new method can be viewed as an alignment with beam constrained tracks which allows to correctly handle correlations between reference sensors with tilted devices under test.

A beam constrained track is a $3 \mathrm{D}$ straight line passing through a hit at a reference sensor, typically the first sensor in the telescope, whose direction is given by the beam axis. The explicit introduction of beam constrained tracks allows to compute the intersections with all other sensors using the current telescope geometry data base. From these predicted intersections, the residuals in the $u$ and $v$ direction 

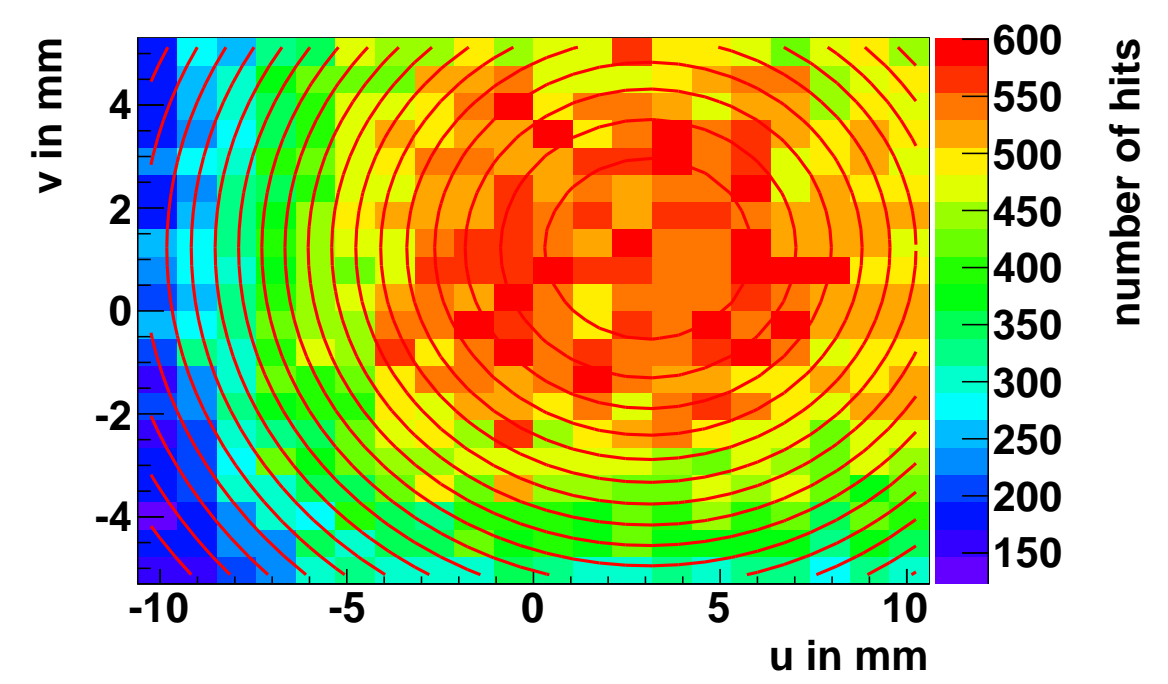

Figure 4.10.: The number of hits on the first Mimosa26 sensor in the telescope. A 2 D Gaussian model is fitted to the local hit distribution to measure the offsets $u_{b}$ and $v_{b}$ between the center of the active sensor area and the beam axis.

with all other hits in the same event are computed. A typical set of residual histograms between the first and the last Mimosa26 sensor is shown in Fig.4.11. The residual histograms contain a flat side band from wrong hit-to-track pairings and a signal peak containing correct pairings. Without telescope misalignment, the residual peaks would be centered around zero for all sensors. This constraint is employed to compute alignment corrections for the sensor shifts $\Delta x$ and $\Delta y$ to center the peaks around zero. A robust truncated average is used to compute the center of residual peaks $u_{0}$ and $v_{0}$. Bins in the side bands with a height below a user defined threshold are discarded in the truncated average. A typical threshold is $>0.5$ times the height of the signal peak. Then, sensor alignment shifts $\Delta x$ and $\Delta y$ can be computed in the same way as for the beam spot correction.

The alignment with beam constrained tracks has the advantage that it does not require the sensor to be large enough to contain the whole beam spot. This is very useful for sensors with a small active
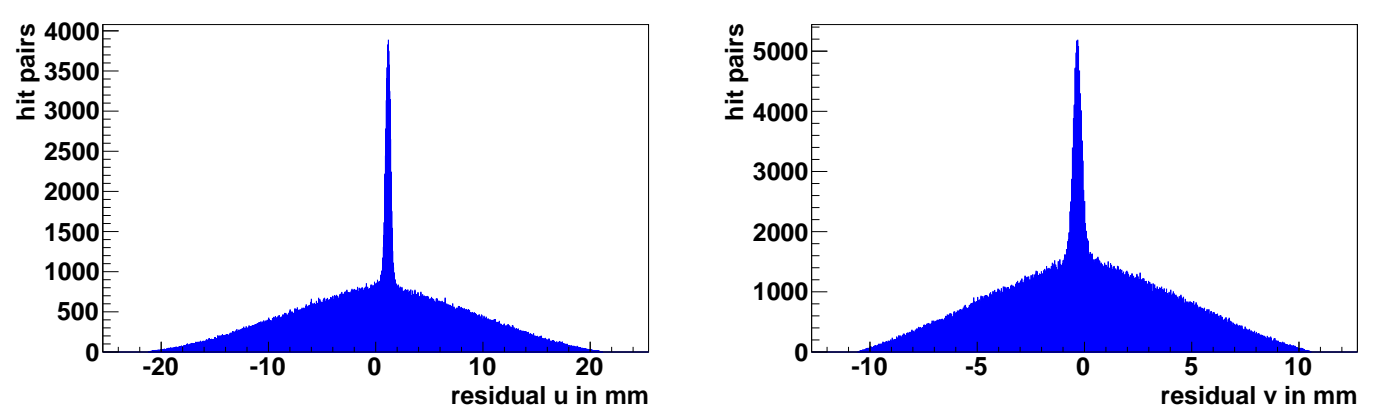

Figure 4.11.: Residuals in the local $u$ and $v$ direction between beam constrained tracks defined at the first sensor in the telescope and hits at the last sensor in the telescope. The full width at half maximum of the residual peak is in the order of $0.6 \mathrm{~mm}$. The position of the signal peaks can be centered around zero by shifting the center of the last sensor in $x$ and $y$ direction relative to the beam axis. 
area as for example for test beams with DEPFET prototype sensors. Furthermore, the alignment with beam constrained tracks offers a way to test the synchronization between the Mimosa26 sensors and the devices under test. If there is no residual peak visible at the devices under test, the run is lost for studies with telescope tracks. That is why it is so important to compute the correlation plots as described here.

The above described methods for telescope pre-alignment are implemented in the DEPFETCorrelator processor. The required inputs are an LCIO run file with hits on all layers, a gear file and the file name of a geometry data base. The processor outputs a new or updated geometry data base with the given file name. After pre-alignment, the $x$ and $y$ shifts of all sensors relative to the beam axis should be known to an accuracy of $\sim 100 \mu \mathrm{m}$. This method suppresses telescope shearing and gives a good starting point for efficient track finding.

\subsubsection{Alignment with tracks: Kalman Alignment Algorithm with annealing}

A well established method is the alignment with tracks [70, 71, 18]. The general idea is to minimize a global $\chi^{2}$ functional as a function of the sensor alignment parameters. The global $\chi^{2}$ function for telescope alignment is the sum of standardized track residuals from a large sample of tracks reconstructed in the telescope.

$$
\chi^{2}=\sum_{j}^{\text {tracks hits }} \sum_{i}^{T} r_{i j}^{T} R_{i j}^{-1} r_{i j}+\left(a-a_{0}\right)^{T} E_{0}^{-1}\left(a-a_{0}\right) \quad \text { where } \quad r_{i j}=\left(m_{i j}-H \lambda_{i j}\right)
$$

Here, $r_{i j}$ denotes the $2 D$ residual at the $i$ th sensor along the beam line for track $j$. The residual covariance $R_{i j}$ matrix can be computed as

$$
R_{i j}=H C_{i j} H^{T}+V_{i j}
$$

The residual covariance matrix weights the residuals according to the statistical errors from the track extrapolation $H C_{i j} H^{T}$ and the errors from the position measurement $V_{i j}$. The second term in Eq.4.22 penalizes deviations of the alignment parameters $a$ from an initial set of alignment parameters $a_{0}$. The initial alignment covariance matrix $E_{0}$ can be used to express a degree of belief on the initial alignment parameters. For our purpose, we will always take $E_{0}$ as a diagonal matrix.

The Kalman Alignment Algorithm with Annealing is an unbiased, sequential method derived from the Kalman Filter to minimize the global $\chi^{2}$ function [18, 71]. It is a sequential method in the sense that it processes tracks one by one and updates the alignment parameters and their covariance matrix after each processed track. The main benefit of this method is that it can be seen as a generalization of the Kalman filter based track fitter developed above. The extension is that now the track residuals depend not only on the track parameters but also on the alignment parameters. The technical details of the implementation are given in appendix $B$

A generic problem for the alignment of beam telescopes are the shearing, torsion and stretching deformations of the telescope. Small global distortions of the telescope leave the track residuals invariant but bias the track parameters, especially the local incident angles of tracks into the sensor planes. However, the build up of large telescope shearing or stretching poses a severe problem for telescope alignment as can be seen for the example of shearing: Shearing of the beam telescope increases the predicted flight 

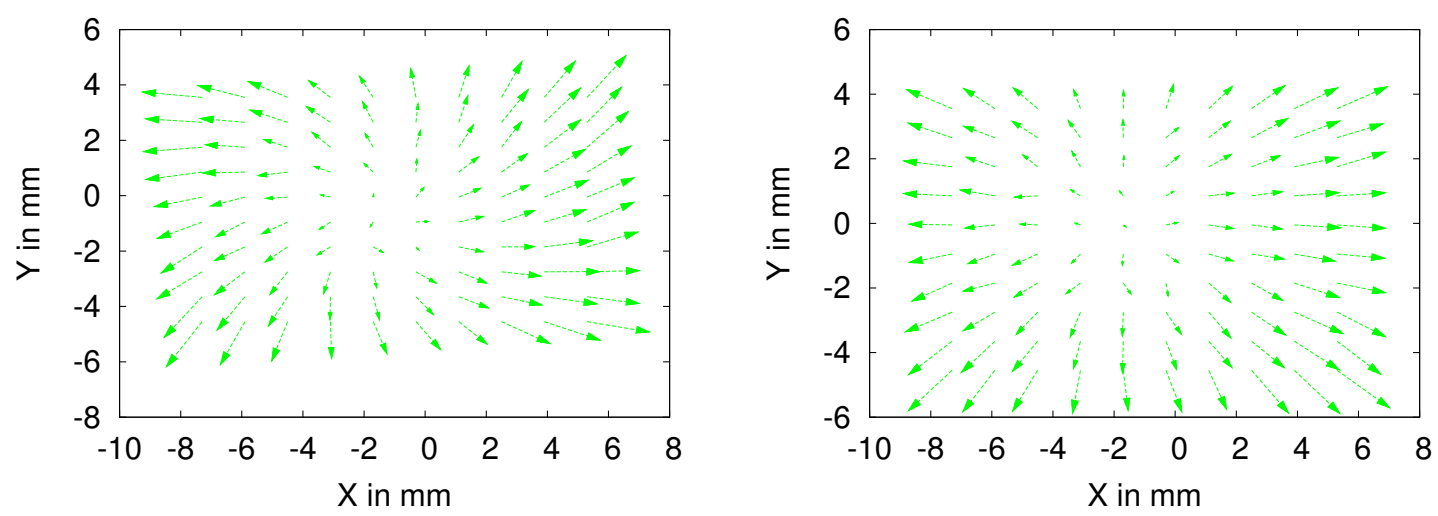

Figure 4.12.: Impact of telescope torsion on reconstructed track directions $(d x / d z, d y / d z)$ at the $z=0$ plane in front of the telescope. The averaged track directions are plotted as a $2 \mathrm{D}$ vector field inside the telescope acceptance. Left: Torsion is visible as an overlaid rotation in the field of track directions. Right: Track directions after correction of torsion using the collimated beam model in track fitting as described above. The beam covariance matrix enforces that track parameters should be uncorrelated in the $x-z$ and $y-z$ plane.

distance of the particle between hits and increases the extrapolation errors of track parameters. Large shearing blows up the track parameter covariance and shrinks the value of standardized residuals in the global $\chi^{2}$ function. In other words, excessive telescope shearing allows to minimize the track residual to zero and ultimately leads to a divergence of track based alignment. We decided to follow two solutions for this problem:

1. In order to avoid large shearing, torsion and stretching distortions, we can assign a zero variance in $E_{0}{ }^{6}$ to the alignment parameters $\Delta x, \Delta y, \Delta z$ and $\Delta \gamma$ of the first and the last sensors in the telescope. This solution effectively freezes the shearing, stretching and torsion which are present in the initial telescope geometry. The alignment parameters $\Delta x, \Delta y, \Delta z$ and $\Delta \gamma$ of the remaining inner sensors are effectively corrected with respect to the position of the two outermost sensors.

2. The collimated beam model sets tight constraints on the average beam direction and the correlations between track incident angles and positions. The collimated beam model allows to align the $\Delta x, \Delta y$ and $\Delta \gamma$ degrees of freedom for all but the first sensor in the telescope. The position of the first sensor should be frozen to the position achieved after pre-alignment.

A main benefit of the second approach is the possibility to suppress torsion in the aligned telescope geometry. The impact of telescope torsion on track fitting is visualized in Fig.4.12. In the first approach, telescope torsion is induced by the initial errors in the $\gamma$ tilts of the first and the last sensor. Typically, these initial errors are in the order of $20 \mathrm{mrad}$ and lead to correlated biases of the track directions in the order of $0.1 \mathrm{rad}$, as shown in the left panel of Fig.4.12. In the second approach, the collimated beam model imposes the further condition that the track parameters of beam particles should be uncorrelated in the $x-z$ and the $y-z$ plane.

The DEPFETKalmanAlignment processor implements a single pass of the Kalman Alignment Algorithm. A gear file defines the telescope setup, a geometry data base file defines the current telescope

\footnotetext{
${ }^{6} \mathrm{We}$ only consider the cases where $E_{0}$ is a diagonal matrix. For any alignment parameter, we have to specify an initial variance. This allows to add information from survey measurements to the alignment.
} 
geometry and a run data file supplies the track data.

\subsubsection{Strategy for telescope alignment and control plots}

The "hands on" experience with telescope alignment during DEPFET test beams has resulted in a number of recommendations for practical telescope alignment. The position of the telescope table should be fine-tuned until the beam spot is clearly visible on all reference sensors. The distances between sensors and the total length of the telescope should be measured at least to an accuracy of $1 \mathrm{~mm}$. The sequence of track finding and track based alignment steps should be iterated at least two times. The recommended sequence is as follows:

- First Finder Pass: After pre-alignment, the track finder should be used with very loose cuts on the track quality. In particular, the maximum allowed residual for adding hits to seed tracks should be around $200 \mu \mathrm{m}$. Cuts on the track $p$-value should be avoided. Even then, the track finder is likely to find only a sub-set of all recorded tracks and there is a chance that tracks have noise hits assigned.

- First Alignment Pass: Due to the limited quality of the track sample, it is advised to restrict the alignment to the most sensitive alignment degrees of freedom in the first pass. In other words, only the $\Delta x, \Delta y, \Delta \gamma$ degrees of freedom should be aligned while all other degrees of freedom should be kept at their starting values.

- Second Finder: After the first alignment with tracks, the track finder should cut on the $p$-value of the track fit to avoid badly reconstructed tracks. A typical cut is to request tracks with $p>0.1$. For telescope alignment, the quality of tracks is generally more important than their total number. After the first alignment pass, the track finder is likely to find more tracks with less contamination from noise hits.

- Second Alignment: After having a good track sample, the alignment with tracks should be repeated. In this second pass, it is recommended to include the $\Delta z$ degree of freedom for all inner sensors into the alignment fit. However, the $\Delta z$ of the first and last sensor should be excluded to avoid telescope stretching. At this stage of the alignment, the sensors tilts $\Delta \alpha$ and $\Delta \beta$ should be included as well. The square root of the initial variance for $\Delta \alpha$ and $\Delta \beta$ should be set to the expected errors during the mechanical installation; typically this error is around $\sim 20 \mathrm{mrad}$.

There are several control plots to judge the quality of telescope alignment from data. The distribution of $p$-values from track fitting after all alignment steps is shown in Fig.4.13. The $p$-value distribution is flat apart from a peak at zero caused by badly reconstructed tracks. There are many possible reasons for bad track reconstruction. One reason is Bremsstrahlung of charged particles in the sensor material leading to a wrong estimation of the particle momentum and multiple scattering deflections. Another potential reason are badly reconstructed hits, or outliers, with large cluster sizes $(>4)$ at the Mimosa26 modules, see Fig. 4.4. It can be expected that the spatial resolution obtained from these hits is far worse than $3.5 \mu \mathrm{m}$. A more refined approach to hit reconstruction for Mimosa26 modules should estimate spatial resolutions separately for small and large cluster sizes. The residual distributions in the $u$ and $v$ directions after alignment were already shown in Fig. 4.5. The remaining misalignment in the telescope leads to systematic shifts of the mean residuals as a function of the track intersection position on the 
4. The reconstruction of beam telescope data: methods, steps and calibration

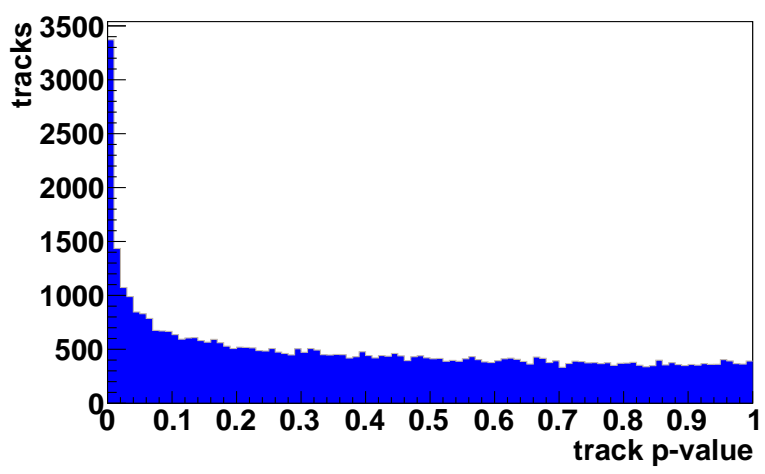

Figure 4.13.: Distribution of track $p$-values in the fully aligned EUDET telescope. The fitted tracks are required to have hits on all Mimosa26 sensors and cover the whole telescope acceptance.
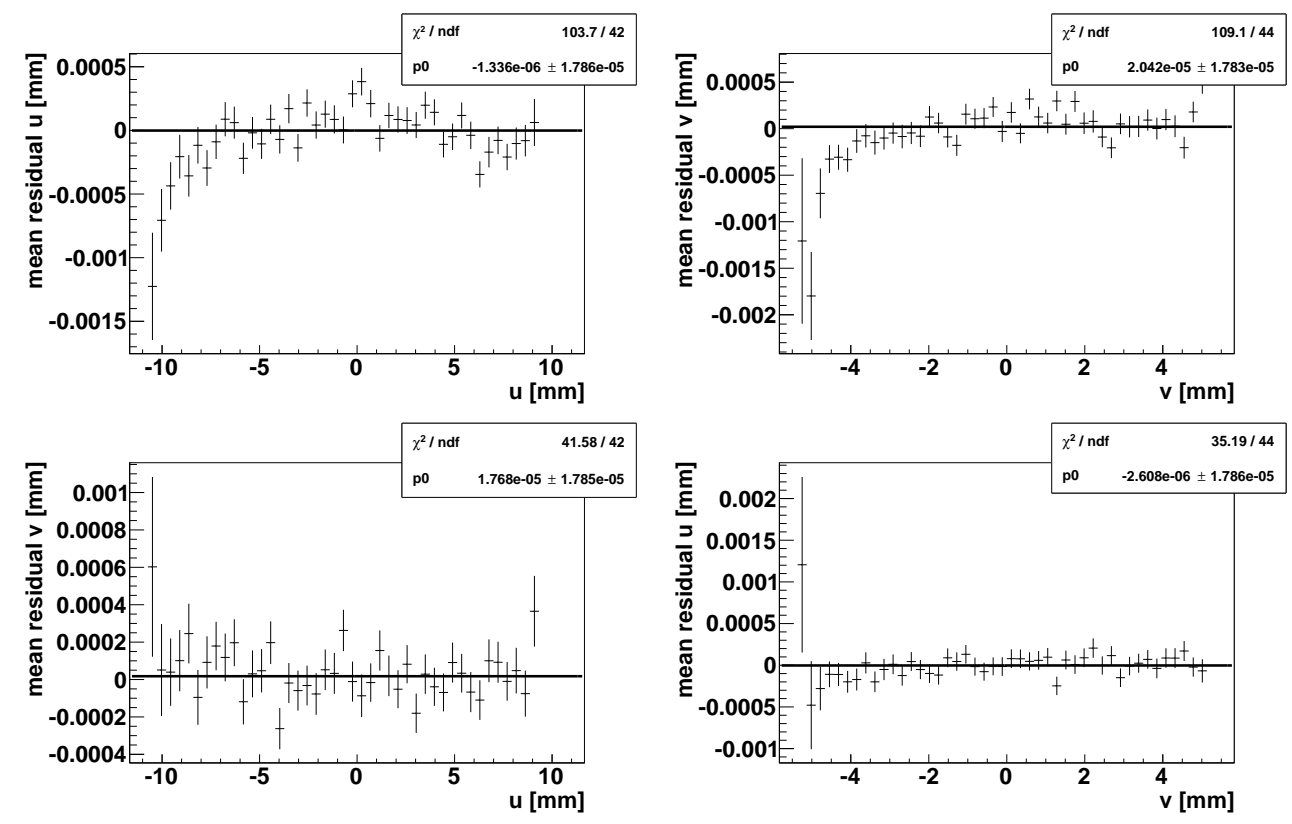

Figure 4.14.: Study of residual shifts on Mimosa26 module No. 2 after alignment. Systematic shifts of the fitted mean residual $p_{0}$ from zero are not observed within statistical errors.

sensor. The resulting four possibilities to study shifts of the mean residual $r_{u}$ and $r_{v}$ along the sensor $u$ and $v$ axis are shown in Fig.4.14. The mean residuals are centered around zero within statistical errors and independent of the hit position on the sensor. 


\section{Thin DEPFET modules in test beams: Validation of the simulation}

\subsection{Introduction to the validation approach and the results}

As already outlined in section 2.3, the present chapter is the third and final step towards the experimental validation of the DEPFET detector simulation. In the first step, covered in chapter 3 , we have presented a simulation algorithm for the detection of particle hits in a module of the Belle II pixel detector. This detector simulation allows to make quantitative predictions for the detected signal charge, the cluster size and the intrinsic spatial resolution needed to study the vertex resolution of the future Belle II experiment at SuperKEKB. In the second step, covered in chapter 4, we have described methods to reconstruct particle tracks in the EUDET beam telescope and to measure the spatial resolution of pixel modules from track residuals. In this chapter now, we will apply these reconstruction methods to DEPFET pixel modules installed in the EUDET beam telescope as devices under test. We present measurements of the detected signals, the intrinsic spatial resolution and the hit detection efficiency. The data obtained during test beams at DESY and CERN SPS in the years 2011-2013 confirms that the DEPFET detector simulation gives a realistic description of the detector response to charged particles.

The first (preparatory) part of the validation study is the offline calibration of the raw data from the DEPFET module. The readout noise is estimated pixel by pixel from the fluctuations of the digitized drain currents. The proposed scheme to calibrate the readout noise in terms of an equivalent noise charge (ENC) is to fit the total detected signals to the well known distribution for energy loss straggling in $50 \mu \mathrm{m}$ silicon. The measured energy loss straggling is well reproduced from the Geant 4 based detector simulation. The measured readout noise is equivalent to $120 \pm 30$ electrons.

The validation focuses on the study of the spatial resolution of the tested pixel modules since this is ultimately the most important variable for a vertex detector. The spatial resolution is measured both for ultra relativistic hadrons, mostly pions, with a momentum of $120 \mathrm{GeV}$ at the $\mathrm{H} 6$ test beam area at CERN SPS and $3 \mathrm{GeV}$ electrons for test beams at DESY. The spatial resolution is measured as a function of the inclination angle of the sensor plane to the particle beam. The angular dependency of the spatial resolution is well reproduced by the simulation.

Test beams with the EUDET telescope provide a unique opportunity to measure the hit detection efficiency of thin DEPFET modules. The pointing resolution of the telescope allows to tag the pixel on the DEPFET sensor which is hit by the particle and should detect signal charge. The measured hit detection efficiency is $>99.5 \%$ over the active sensor area using a low seed signal threshold of 525 electrons. In order to provide a full picture, the efficiency is studied as a function of the seed signal threshold applied in clustering. A significant hit detection inefficiency is measured for thresholds above 1000 electrons.

The very high pointing resolution of the EUDET telescope is exploited to study the charge sharing 
properties of thin sensors on an in-pixel level. For this purpose, large samples of about 1 million high energy pions hitting the DEPFET sensor at perpendicular incidence were recorded. The data is converted into a map of the average detected signal charge against the in-pixel impact position of the particle. The map of the mean cluster size and seed signal reveal border regions between pixels with enhanced charge sharing in agreement with the detector simulation. The measured mean seed signal drops from about 4500 electrons for tracks hitting the geometrical center of the pixel area to 1600 electrons for tracks hitting the pixel corners. The dependence of the mean seed signal and the mean cluster size on the impact position of the track is a consequence of signal charge diffusion during charge collection. Diffusive charge sharing splits the total signal charge created by the charged particle in $50 \mu \mathrm{m}$ of fully depleted silicon between multiple pixels. Maps of the in-pixel hit detection efficiency confirm that the efficiency drops below $99 \%$ precisely in the pixel corners even at very low seed signal thresholds.

This chapter is structured as follows: Section 5.2 gives a brief overview about the PXD6 sensors and the test system used for test beams. The next section 5.3 covers the offline corrections necessary to calibrate the sensor raw data and to obtain zero suppressed digits for clustering. The results of the validation measurement are presented in section 5.4 .

\subsection{Overview of prototype sensors and the test system}

Test beam campaigns of the DEPFET Collaboration from mid 2011 to 2013 were focused on the validation and testing of the latest DEPFET sensor production (PXD6). The PXD6 production is a first test run to proof the feasibility of the sensor design and readout concept for the pixel detector at Belle II. The sensors are $50 \mu \mathrm{m}$ thick with pixel cell sizes of $50 \times 50 \mu \mathrm{m}^{2}$ or $50 \times 75 \mu \mathrm{m}^{2}$. The test system uses close to final front-end ASICS (DCDB/SWB). For this reason, the tested prototype modules implement crucial parts of the final detector concept of the Belle II pixel detector.

The Belle II specific sensor layout has a double pixel structure with shared source implantation and pixel sizes of $50 \times 50 \mu \mathrm{m}^{2}$ (first layer) or $50 \times 75 \mu \mathrm{m}^{2}$ (second layer). Fast charge collection ( $<100 \mathrm{~ns}$ ) is provided by the implantation of a novel drift region in the pixel cell. This drift region is introduced to ensure efficient charge collection for particles traversing the sides or corners of the pixel cell. The sensor substrate is a high resistivity n-type silicon with a $p^{+}$backside contact. The substrate is thinned down to $50 \mu \mathrm{m}$ below the active area. The negative backside bias voltage needed to deplete the sensor is applied via a punch through mechanism from the front side. The size of the DEPFET transistor remains similar to previous ILC type productions with small pixel areas. The typical length of the transistor gate in the current technology is $4-6 \mu \mathrm{m}$. The figure of merit is the $g_{q}$ factor, which is the internal signal amplification gain of the device. The internal gain expected for the Belle II sensors is around $500 \mathrm{pA} / \mathrm{e}$. In this case, a signal charge of 3200 electrons collected in the internal gate of a pixel amplifies the sampled drain current by $1.6 \mu \mathrm{A}$. Two sensors with Belle II specific designs were successfully pretested and optimized for beam test measurements at CERN in June and October 2012 and at DESY in May 2013. A summary of the module parameters is given in Tab.5.1.

The test system developed for test beams with DEPFET prototypes is shown in Fig. 5.1. The PCB board accommodates a small PXD6 sensor with 32 columns and 64 rows connected to a multi-channel ADC chip (DCDB) and one SWB chip for the control of clear and gate lines. The DHP chip is replaced by a dummy chip (DCDB-RO) to provide a silicon adapter chip for communication with the DCDB 


\begin{tabular}{|l|c|c|c|c|c|c|}
\hline \multirow{2}{*}{ Module } & \multicolumn{2}{|c|}{ number of pixel } & \multicolumn{2}{c|}{ pitch $[\mu \mathrm{m}]$} & area $\left[\mathrm{mm}^{2}\right]$ & gate length \\
\cline { 2 - 8 } & in $u$ [cols] & in $v$ [rows] & in $u$ & in $v$ & $(u \times v)$ & in $\mu \mathrm{m}$ \\
\hline \hline Module A & 32 & 64 & 50 & 75 & $(1.6 \times 4.8)$ & 5 \\
\hline Module B & 32 & 64 & 50 & 75 & $(1.6 \times 4.8)$ & 5 \\
\hline
\end{tabular}

Table 5.1.: Overview of the two pixel modules measured in test beams. The sensors are thinned to $50 \mu \mathrm{m}$ and have the Belle II specific design. The two test modules are equipped with DCDB and SWB readout chips and were pre-selected and optimized before the beam test.

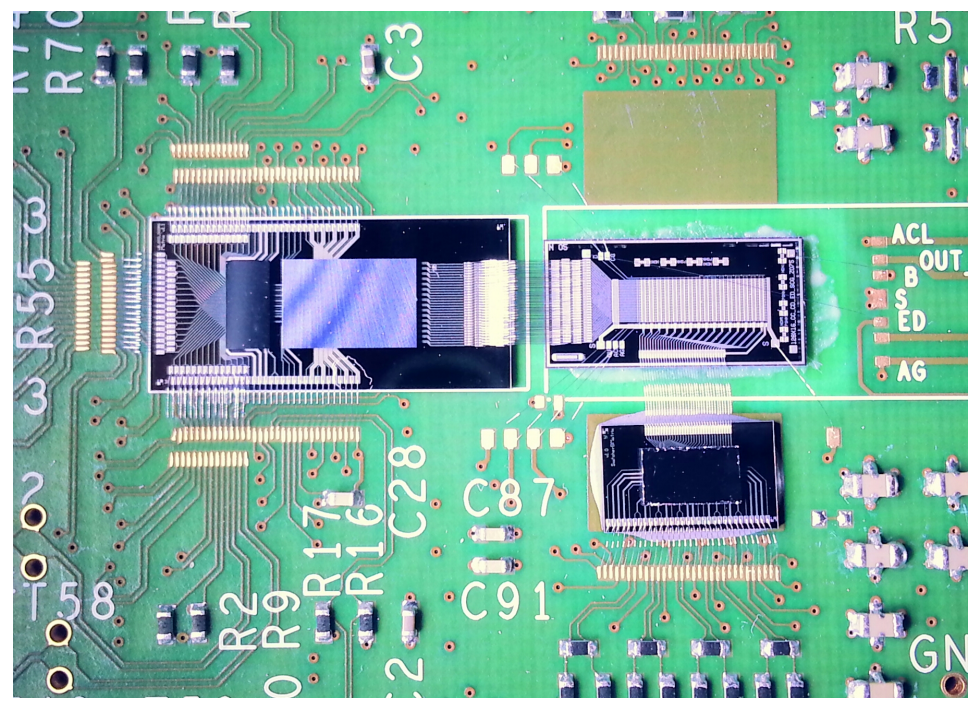

Figure 5.1.: Close up of the system developed for sensor testing. The 2048-pixel sensor in the center is surrounded by the auxiliary ASICS needed to operate it. The DCDB and DCDB-RO ASICS are visible left and the Switcher-B ASIC below the sensor.

chip. A second PCB board with a XILINX FPGA performs event building and sends data via USB link to the DAQ system. The powering of the DEPFET module is done by using a first prototype version of the final DEPFET power supply for Belle II [72].

The readout of the DEPFET pixel module with the DCDB and SWB chips and the calibration measurements are described in detail by Knopf [38]. The 64 rows on the sensor are divided into 16 so-called gates consisting of four rows having common gate and clear terminals (4-fold readout). The integration time needed to sample and digitize the drain currents from all 16 gates is $4.8 \mu \mathrm{s}$. The integration time per gate is $300 \mathrm{~ns}$ which is roughly a factor of three slower than required for Belle I[! The DCDB chip performs an 8 bit digitization of the drain currents from all pixels of a gate in parallel. The drain currents from DEPFET pixels are in the order of $60 \mu \mathrm{A}$ with pixel-to-pixel variations below $10 \%$. The least significant bit (LSB) is $0.1 \mu \mathrm{A}$ and the dynamic range of the ADC is around $24 \mu \mathrm{A}$. A global current subtraction at the input pads of the DCDB allows to shift all drain currents into the dynamic range of the ADC. The default readout mode for the test beams described here is a single sampling of the drain currents followed by a clear of all charges in the internal gate. This readout mode is called single sampling and allows a reduction of the integration time by a factor of two compared to the previously used correlated double sampling readout mode [73].

\footnotetext{
${ }^{1}$ The second version of the DCDB chip was tested to work at the nominal clock frequency of $320 \mathrm{MHz}$ required to read and clear a gate in $100 \mathrm{~ns}$ as needed for the Belle II pixel detector. The test beam results for module operation at $320 \mathrm{MHz}$ are still under preparation.
} 


\section{Thin DEPFET modules in test beams: Validation of the simulation}

The data acquisition of the DEPFET module is controlled by the EUDAQ run control and is synchronized with the rest of the telescope by the trigger logic unit of the EUDET telescope. The digital part of the DCDB receives the TLU trigger and selects event data from a ring buffer. The event data comprises a full sample of the digitized drain currents for all $32 \times 64$ pixels called a frame. The event data from the DEPFET module is sent to a data collector running on the EUDAQ PC and is saved in the same file as the data from Mimosa26 sensors. The integration of the DEPFET system into the EUDET telescope is well tested in a series of previous test beams and described in detail by Furletov [74, 75, 76]. The final test of data integrity and synchronization is the observation of correlations between telescope tracks and DEPFET hits during the offline data reconstruction as explained in section 4.5.4.1.

\subsection{Offline calibration of sensor raw data}

The offline calibration of raw signals is performed by a dedicated Marlin processor (DEPFETPedestalNoiseProcessor) as a further step of data reconstruction before clustering. For the Belle II pixel detector, the calibration of raw signals from the DCDB will be performed on the fly using one further ASIC, the Data Handling Hybrid [43]. The calibration consists of a pedestal and common mode subtraction of the raw signals from the DCDB chip on the test system. The required calibration constants - pedestal, noise and bad pixel maps - are directly calculated from the triggered raw signals collected during a data taking period or run.

Pedestals are cyclically re-calculated every 500 - 1000 triggers because drain currents may drift with the ambient temperature $2^{2}$. After the pedestal subtraction, a common mode correction is performed to subtract a common noise offset in the drain currents from pixels in the same gate. Finally, a user defined zero suppression threshold is applied to filter the signal from pixels which have detected a particle hit. Fig. 5.2 shows the steps of the offline calibration for a typical event from a test beam run at DESY. After offline calibration, a hit from a $3 \mathrm{GeV}$ electron is visible near the center of the active sensor area.

The computation of calibration constants is illustrated in Fig. 5.3. The digitized drain current from the PMOS transistor on a pixel cell is the raw signal. The following statistical model is used to detect signal charge in the time series of raw signals from a single pixel:

$$
\text { RawSignal }(t)=\text { Pedestal }(t)+\text { CommonMode }(t)+\text { Noise }(t)+\text { Charge }(t)
$$

The variable $t$ numbers the triggers during a run. The raw signals fluctuate around a pedestal value. The pedestal is estimated pixel by pixel as a long time average of several hundred consecutive events. The pedestal is expected to define a stable baseline value. After the pedestal subtraction, mostly noise or sometimes signal charge from a particle hit remains. Signal charge is detected as large excursions or outliers above the pedestal value.

To organize the calculation of calibration constants, pedestal and pixel noise, we split the run data into batches of several hundred events. A batch of events is split into two sub-batches of equal size, followed by two loops or passes to obtain the calibration constants:

- First Pass: Pedestal and noise values are computed as the mean and standard deviation of raw signals in the first sub-batch of events. Raw signals with signal charge from particle hits enter the

\footnotetext{
${ }^{2}$ The test system has no cooling system and a settling time is needed until the temperature of the test system is stabilized and data taking starts.
} 

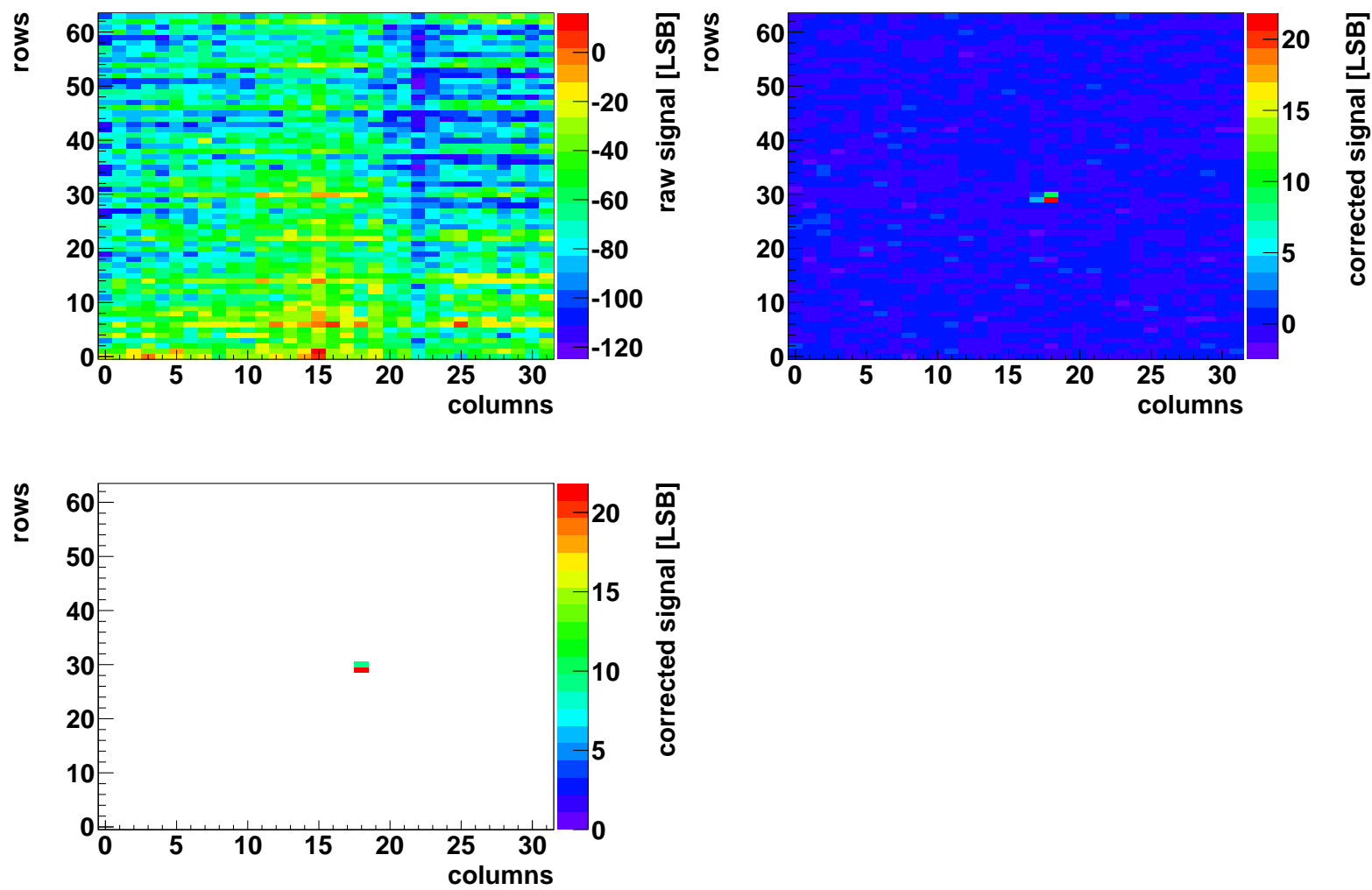

Figure 5.2.: Offline calibration of raw signals from a DEPFET module. The calibration of the full data from one event starts with the $32 \times 64$ matrix of raw signals (upper left). The first calibration step is the pixel-by-pixel subtraction of pedestal and common mode and results in a matrix of corrected signals (upper right). The second step is the sparsification of signals by a zero suppression threshold (lower left). A hit from a $3 \mathrm{GeV}$ electron is visible as a cluster around row 29 and column 18.

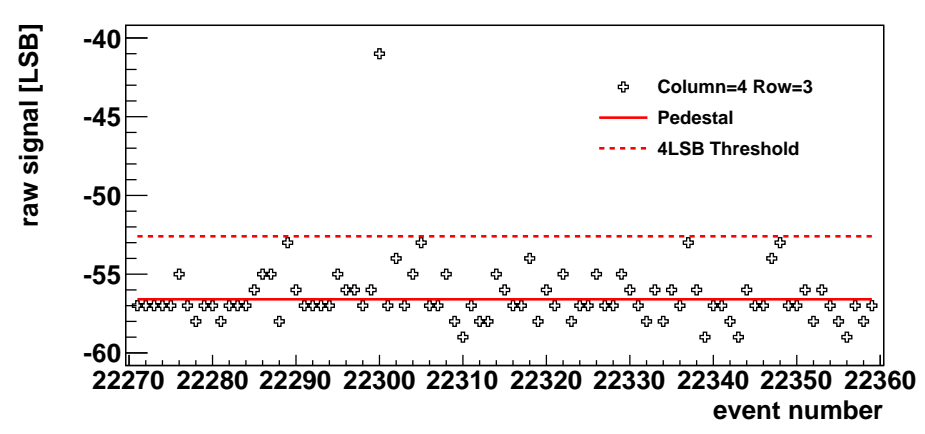

Figure 5.3.: Raw signals from a single pixel from 100 consecutive events during a test beam run at DESY. At a nominal trigger rate of $1 \mathrm{kHz}$ the time series covers an interval of $100 \mathrm{~ms}$. The raw signals are measured in units of the least significant bit (LSB) of the ADC chip. The output range of the ADC chip is -127 to +128 . The estimated pedestal values and a 4 LSB threshold for hit detection are shown as well. The fluctuation of raw signals around the pedestal contains contributions from common mode noise and pixel noise. A hit from a $3 \mathrm{GeV}$ electron is detected at event number 22300 . 


\section{Thin DEPFET modules in test beams: Validation of the simulation}

averaging and bias the pedestal to more positive values. The common mode offset fluctuates from trigger to trigger and contributes to the estimated noise value.

- Second Pass: The first pass delivers an intermediate estimate for the pedestal and noise values. This first pass pedestal value defines a baseline for hit detection. The second pass pedestal is computed as the mean of raw signals in the second sub-batch excluding detected hits. The noise value is computed as the standard deviation of charge values.

The common mode is a common offset of all drain currents which are sampled and digitized at the same time. In the 4-fold readout scheme for Belle II, the PMOS transistors of all pixels in four rows have a common gate terminal and are switched at the same time. The common mode is computed as

$$
\text { CommonMode }=\frac{\sum_{i}(\text { RawSignal }(i)-\text { Pedestal }(i))}{N_{C M}}
$$

where the index $i$ runs over all $N_{C M}$ pixels in a 4-fold row (gate) which are not masked and not hit. The possibility to mask pixels during a run period allows to ignore very noisy or damaged pixels and helps to make the common mode more robust. Hit pixels are excluded event by event to avoid biasing the common mode. A raw signal is flagged as hit, if the raw signal exceeds a 4 LSB threshold, see also Fig. 5.3. The charge value is obtained after pedestal and common mode subtraction

$$
\text { Charge }=\text { RawSignal }- \text { Pedestal }- \text { CommonMode } .
$$

The charge value is directly proportional to the number of electrons collected in the internal gate. The conversion factor or gain $g_{q}$ will be determined in the next section. The zero suppression discards all charge values below a user defined threshold as noise. The default choice for the zero suppression threshold is 3 LSB. After zero suppression, digits are built as triplets consisting of the charge value and the column and row address of the pixel. These digits are written to the LCIO run file for hot pixel masking and clustering as described in section 4.3 .

The noise and pedestal distribution measured during the test beams can be seen in Fig.5.4. The noise and pedestal values are measured in units of the least significant bit (LSB) which is around $0.1 \mu \mathrm{A}$ according to calibration measurement by Knopf [38]. The most probable value of the pixel noise is 0.6 LSB for both tested modules, compatible with results obtained during laboratory characterization [38]. The central value of the pedestal distribution can be shifted by a global current source at the input pads of the DCDB chip. A good working point is found if the digitized drain currents of all pixels are inside the output range of the ADC going from -127 to +128 . There are several factors that can influence the variation of pedestals on the sensor: pixel to pixel variation of the gate length, variations of the deep $n$ implantations below the gate and variations between the gains of different ADC channels on the DCDB chip. The measured variation (RMS) of pedestals is around 30 LSB which is equivalent to a drain current variation of $3 \mu \mathrm{A}$.

Fig. 5.5 shows that the noise maps of Module A and B are rather flat over the sensor area while the noise is a factor two higher at the outermost columns. The variation of pedestals over the sensor area is shown in Fig.5.6. In order to make the validation study more robust against effects from prototypes, we mask pixels having a very high noise $(>1.5 \mathrm{LSB})$ and pixels having a pedestal near the end of the dynamic range of the ADC. 

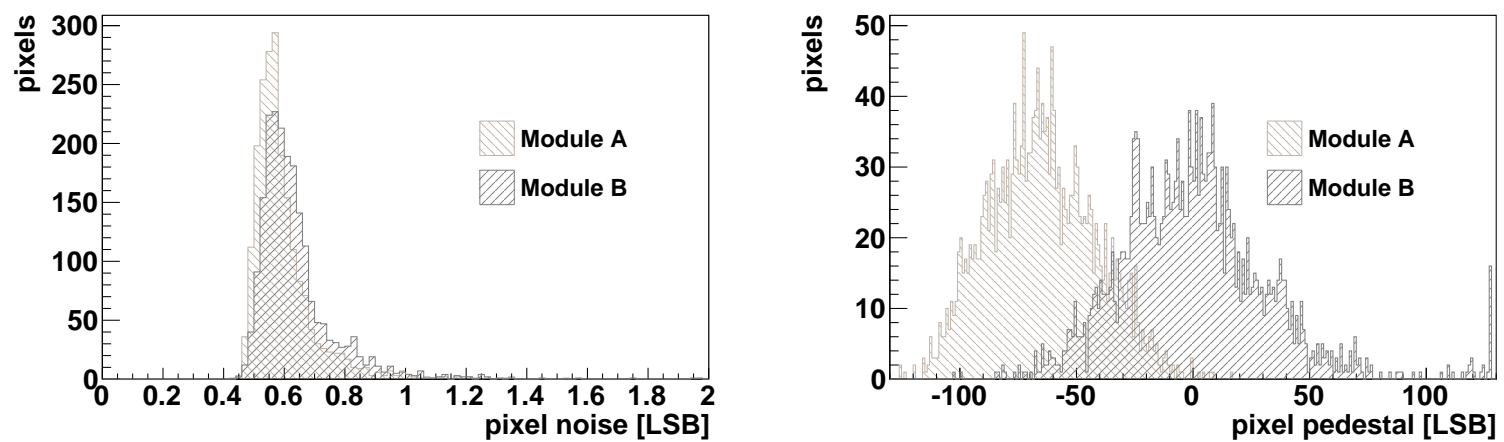

Figure 5.4.: Left: Measured pixel noise distribution for Modules A and B after pedestal and common mode correction. Noise values are measured in units of the least significant bit (LSB) of the ADC chip. The most probable noise value is $0.6 \mathrm{LSB}$. Right: Distribution of pedestal values for Modules A and B. The measured standard deviation of pedestals is 23 LSB (Module A) and $32 \mathrm{LSB}$ (Module B). All pedestal fit into the dynamic range of the ADC chips ranging from -128 LSB to 127 LSB.
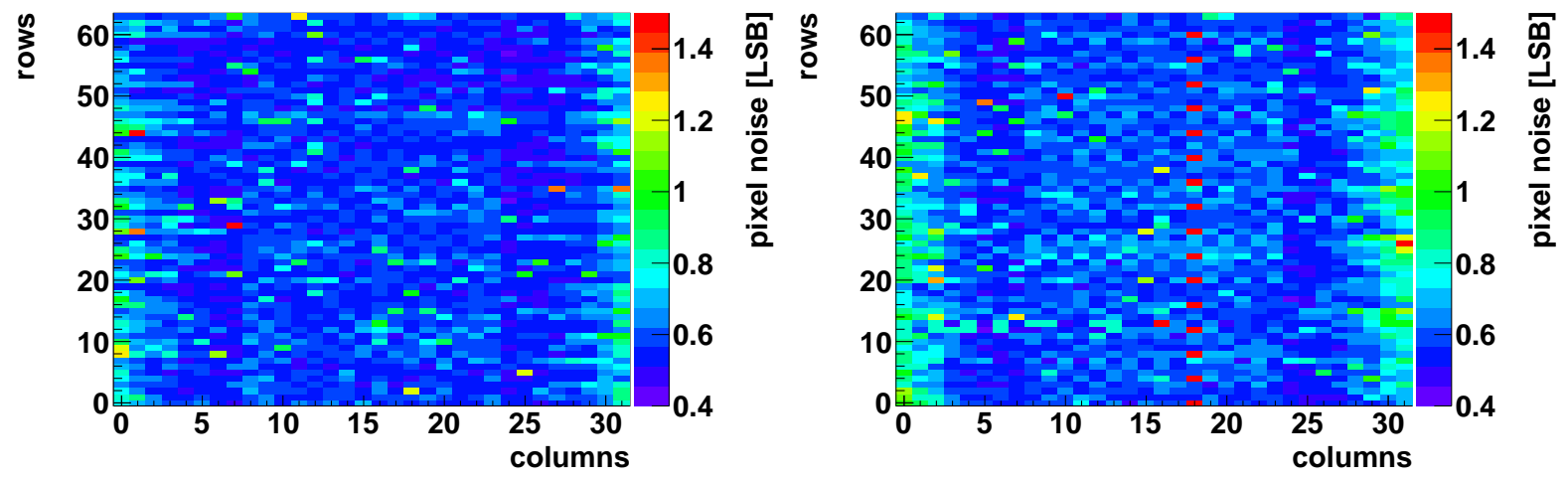

Figure 5.5.: Map of measured pixel noise for Module A (left) and Module B (right). Noise values are measured in units of the least significant bit (LSB) of the ADC chips. The noise in the outermost columns is a factor of two larger compared to the noise in the inner columns. Problems with an individual ADC channel appear as a period four pattern of noisy pixels in the same column. 

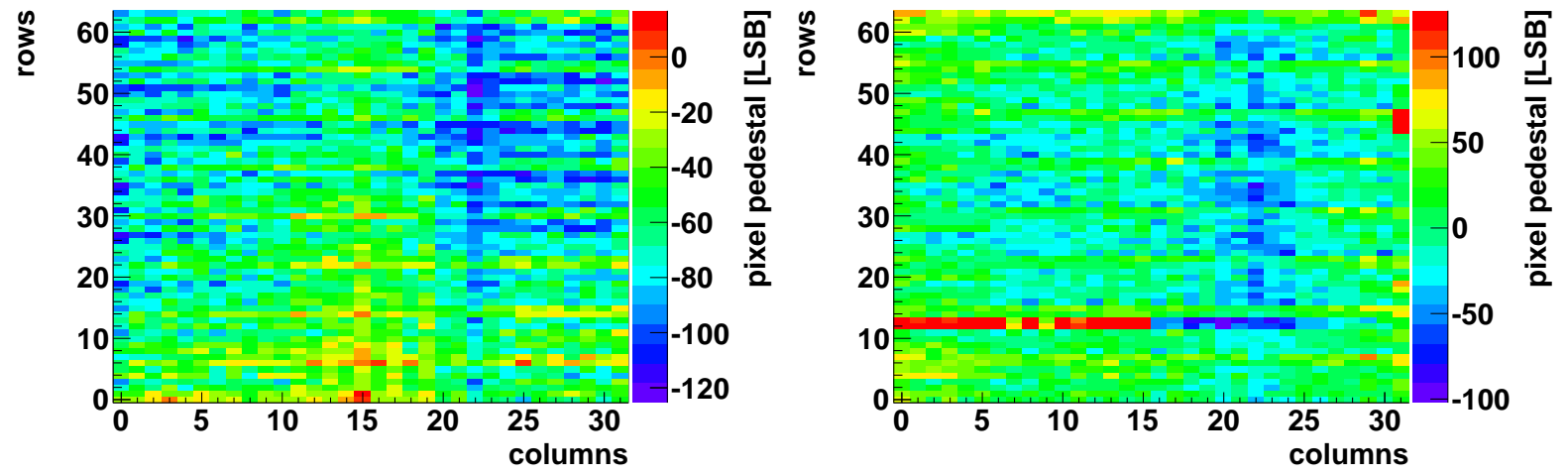

Figure 5.6.: Map of measured pedestals for Module A (left) and Module B (right). Pedestals are measured in units of the least significant bit (LSB) of the ADC chip. The variation of pedestals is proportional to the variation of drain currents from the DEPFET transistors. Rows $12-15$ of Module B show very large pedestals and are masked for offline data processing.

\subsection{Results of the validation measurements}

To be useful for vertexing, the DEPFET modules must measure the intersection points of charged particles with high spatial resolution and high efficiency. For test beams, especially the following questions are of interest:

- What is the probability to detect a signal cluster at the predicted particle impact point? This probability is called the hit detection efficiency $\epsilon$.

- What is the distribution of detected cluster signals and cluster sizes for a particle intersection? The key to a high spatial resolution are compact clusters of $2-3$ pixels and a high signal to noise ratio.

- What is the distribution of position measurement errors on the sensor, especially its standard deviation called the intrinsic spatial resolution?

- How is the signal charge transported into the internal gates and shared between neighbouring pixels? The modeling of the signal transport was the key point in the detector simulation.

Following these questions, the purpose of this section is to validate the detector simulation by comparing the simulated with the real detector response. With the above described and developed steps and algorithms, we are now in a position to relate the detector response to the properties of the charged particle at the sensor from an experimental point of view:

- The offline calibration delivers digits from the DEPFET module for each triggered telescope event.

- The hit reconstruction leads to clusters and (local) hits on the sensor.

- The track finding attempts to match these hits with telescope tracks.

- The track fitting gives a precise estimate of the local track parameters and their covariance. 


\section{Thin DEPFET modules in test beams: Validation of the simulation}

In more formal terms, we can express the detection of a particle intersection at the DEPFET module by the following map:

$$
\left[m, q, p, u, v, t_{u}, t_{v}\right] \stackrel{\text { detector }}{\longrightarrow}\left[s_{1}, \operatorname{row}_{1}, \operatorname{col}_{1} ; \ldots ; s_{n}, \text { row }_{n}, \operatorname{col}_{n}\right]
$$

The numbers on the left hand side are the particle's mass $m$, charge $q$, momentum $p$ and the four local track parameters. The right hand side lists all $n$ pixels responding to the particle intersection with their column and row addresses and the detected signals $s_{i}$. The mapping can be studied experimentally by using tracks from the EUDET telescope or with the detector simulation as described in the case study in section 3.4. The approach to validate the simulation is to compare distributions for variables like the cluster size $n$ or the cluster signal $\sum s_{i}$ between measurements at test beams and simulations. For test beams at DESY and CERN, the mass, charge and momentum of beam particles is of little importance for the detector response due to the high beam momentum and the small sensor thickness. The validation study focuses on the DEPFET specific part of the detector simulation covering the collection of signal electrons in the internal gate of one or more pixel cells. Charge collections is important for the charge sharing between neighbouring pixels and the intrinsic spatial resolution of the sensor. The settings of the detector simulation are summarized in Tab.3.1.

\subsubsection{Study of signal and cluster size distributions}

The detector simulation measures signal in units of electrons collected in the internal gate while the prototype detectors measure signal in units of the least significant bit (LSB). In order to compare data from simulations and test beams, it is necessary to calibrate the value of the LSB in terms of an equivalent number of electrons. Our approach to signal calibration uses the simulated cluster signal in response to beams of $120 \mathrm{GeV}$ pions (CERN) or $3 \mathrm{GeV}$ electrons (DESY) at perpendicular incidence as a standard. The cluster charge is a well understood observable which mostly depends on the simulation of energy loss in $50 \mu \mathrm{m}$ thin silicon in the Geant4 [44] part of the detector simulation. The theoretical error of the most probable energy loss in Geant 4 is $<10 \%$ [50] and justifies the choice of the standard. Calibration measurements of the tested modules using transition lines of radioactive sources are not yet published but used to yield similar results in the past.

The simulated cluster charge distributions for $120 \mathrm{GeV}$ pions at CERN and $3 \mathrm{GeV}$ electrons at DESY are shown in Fig. 5.7 The most probable cluster charge raises from 3300 electrons at DESY to 3500 electrons at CERN. The width of the cluster charge (the Full Width at Half Maximum or FWHM) is 2200 electrons both for pion and electron beams. The differences in the energy loss between electron beams at DESY and pion beams CERN is negligible for thin DEPFET sensors and allows to directly compare test beam data from DESY and CERN. The estimated calibration factor $g$ is $195 \pm 10$ electrons per LSB for Module A and $175 \pm 10$ electrons per LSB for Module B. The calibration factor $g$ is closely related to the internal gain $g_{q}$ of the integrated first amplifier stage. Using the calibration of the ADC channels of $0.1 \mu \mathrm{A}$ per LSB obtained in [38], the internal gain $g_{q}$ is $510 \pm 25 \mathrm{pA} / \mathrm{e}$ for Module A and $570 \pm 25 \mathrm{pA} / \mathrm{e}$ for Module B. The estimated internal gain for the tested modules is close to the expectation for Belle II of $500 \mathrm{pA} / \mathrm{e}$. The gain difference between Module A and B is most likely caused by using different bias voltages for Module A and B during the test beam measurements. The most probable pixel noise of 0.6 LSB measured during test beams translates into an equivalent noise charge of 120 electrons for 

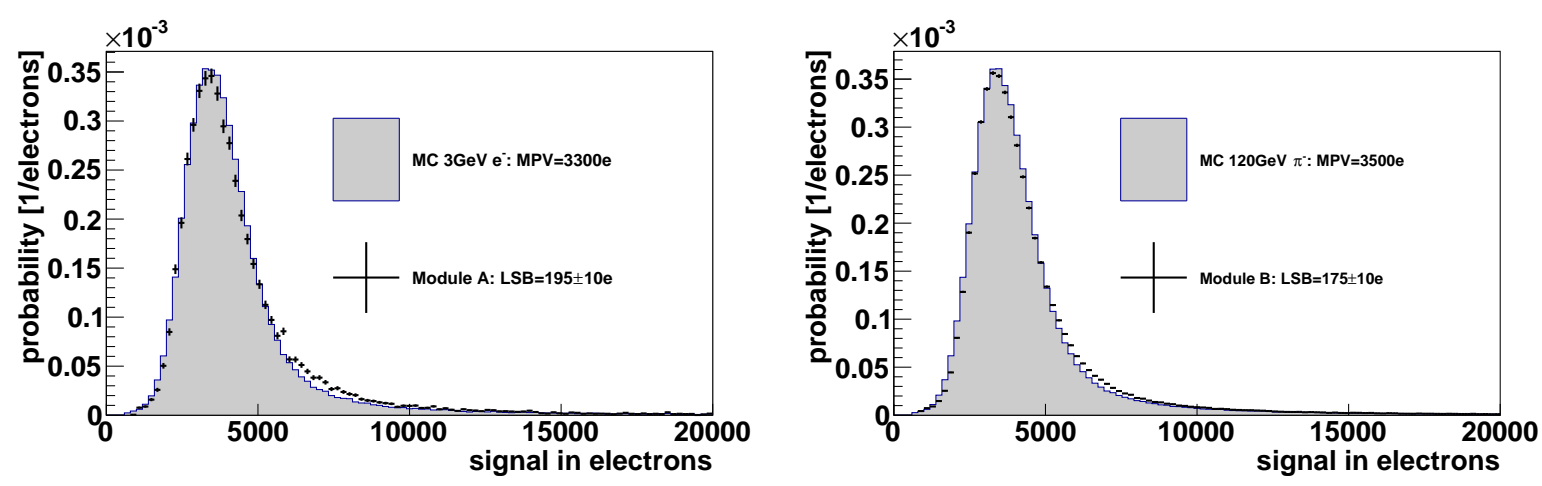

Figure 5.7.: Signal collected in clusters of DEPFET pixels in response to perpendicularly incident $120 \mathrm{GeV}$ pions in the H6 area of the CERN SPS (right) and $3 \mathrm{GeV}$ electrons at DESY (left). The Monte Carlo (MC) prediction drawn in the same Figure corresponds to the DEPFET detector simulation. The histograms are normalized to unit area.

Module A and 105 electrons for Module B. The error on the noise calibration is in the order of $\sim 10 \%$ dominated by the width (FWHM) of the pixel noise histograms shown in Fig. 5.4.

The uniformity of the detector response from different parts of the sensor area after the signal calibrations is studied in Fig.5.8. The uniformity of the mean seed signal for hits in different columns or rows is a direct measure of the uniformity of signal gains. The observed variation of the mean seed signal for different rows or columns is below \pm 100 electrons around the global mean value of 3130 electrons. The gain uniformity is considered as satisfactory and no attempt is made to obtain a pixel-by-pixel fine calibration of signal gains.

Module A was subject to a study of the detector response for different tilt angles during a test beam at DESY with $3 \mathrm{GeV}$ electrons. The dependence of the cluster charge and the cluster size as a function of the incident angle $\theta=\arctan t_{v}$ of the incoming particle is shown in Fig. 5.9. The incident angle in the other direction $\arctan t_{u}$ is very close to zero due to the small beam divergence of roughly $1 \mathrm{mrad}$ (see section 4.4.2.2). The flight length of the particle in the sensor volume increases with the incident angle and more signal charge is created in the sensor. At the same time, the signal charge is shared over more pixel rows on the sensor and the cluster size increases. Both effects are qualitatively reproduced by the detector simulation. According to the simulation, the most probable cluster charge increases from 3300 electrons at perpendicular incidence $\left(\theta=0^{\circ}\right)$ to 5000 electrons at the maximum incidence angle of $\theta=46^{\circ}$. The most probable cluster charge at perpendicular incidence serves as a standard for the calibration of the detector signal. The calibrated cluster signal at $46^{\circ}$ peaks at 4600 electrons and shows a $10 \%$ deviation between test beam data and simulation. At perpendicular incidence, the cluster size depends on the diffusion of signal electrons in the sensor volume. The simulation overestimates the fraction of size one clusters and underestimates the fraction of size two clusters by $4 \%$. The agreement improves for larger tilt angles because the cluster size increasingly depends on the projection of the track length in silicon on the sensor surface.

\subsubsection{Study of the intrinsic spatial resolution}

The intrinsic spatial resolution of the DEPFET prototypes is measured by comparing the center of gravity of clusters to the reference hit position provided by the telescope. The distribution of these track residuals 

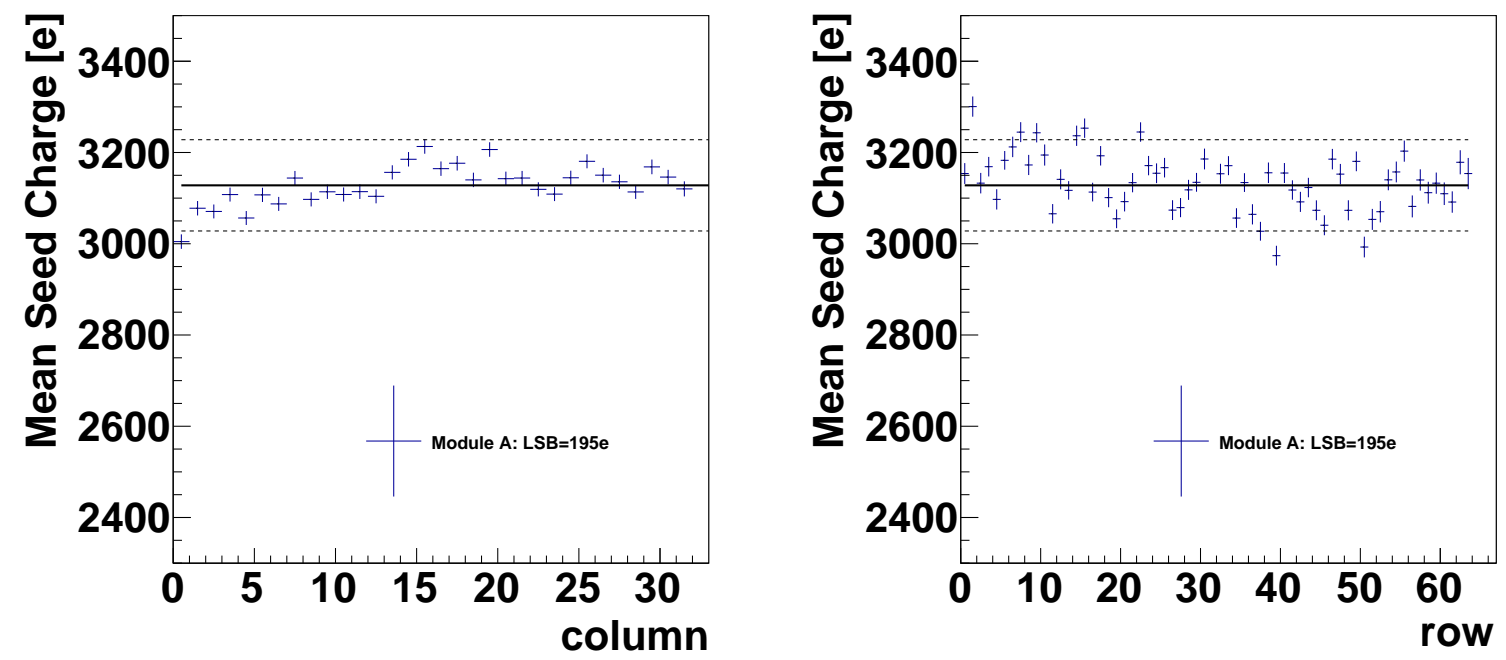

Figure 5.8.: Profile of the mean seed charge for individual columns (left) and rows (right) of Module A exposed to $120 \mathrm{GeV}$ pions at CERN. The global mean seed charge averaged over all pixel columns or rows is equal to 3130 signal electrons. The variations of the mean seed signal per column (row) is sensitive to variations of pixel gains $\left(g_{q}\right)$ and channel by channel variations on the ADC chip (DCDB). The observed variation of the mean seed signal is smaller than \pm 100 electrons.
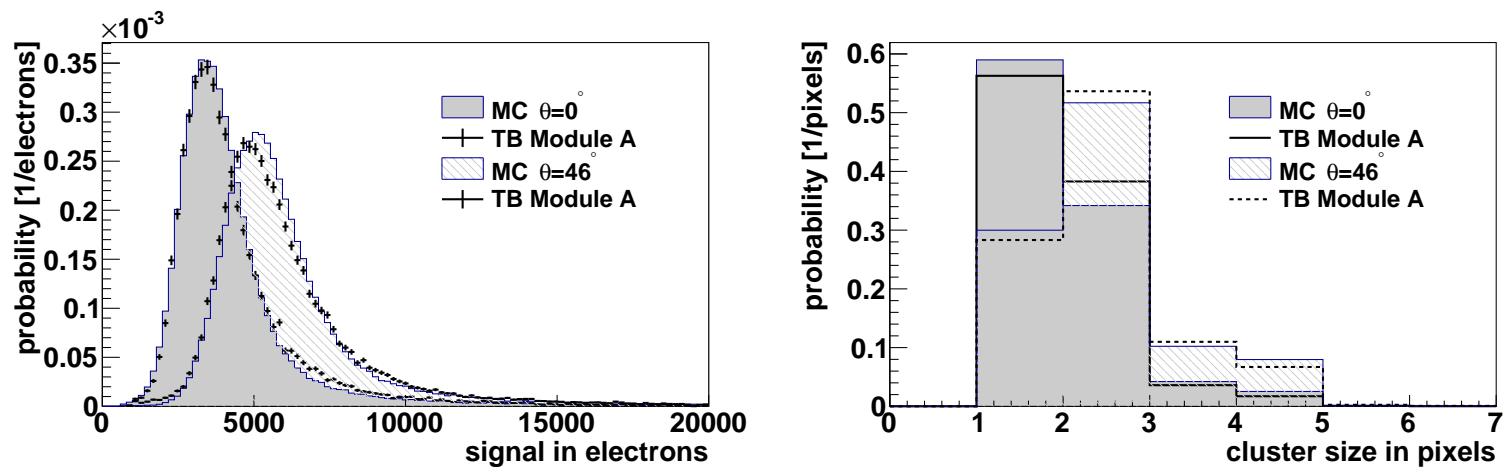

Figure 5.9.: Cluster signal (left) as a function of the incident angle $\theta=\arctan t_{v}$ of the incoming particle. The incident angle in the other direction $\arctan t_{u}$ is close to zero. The cluster signal (left) and cluster size (right) increases with the incident angle as expected. Histograms are normalized to unit area. 

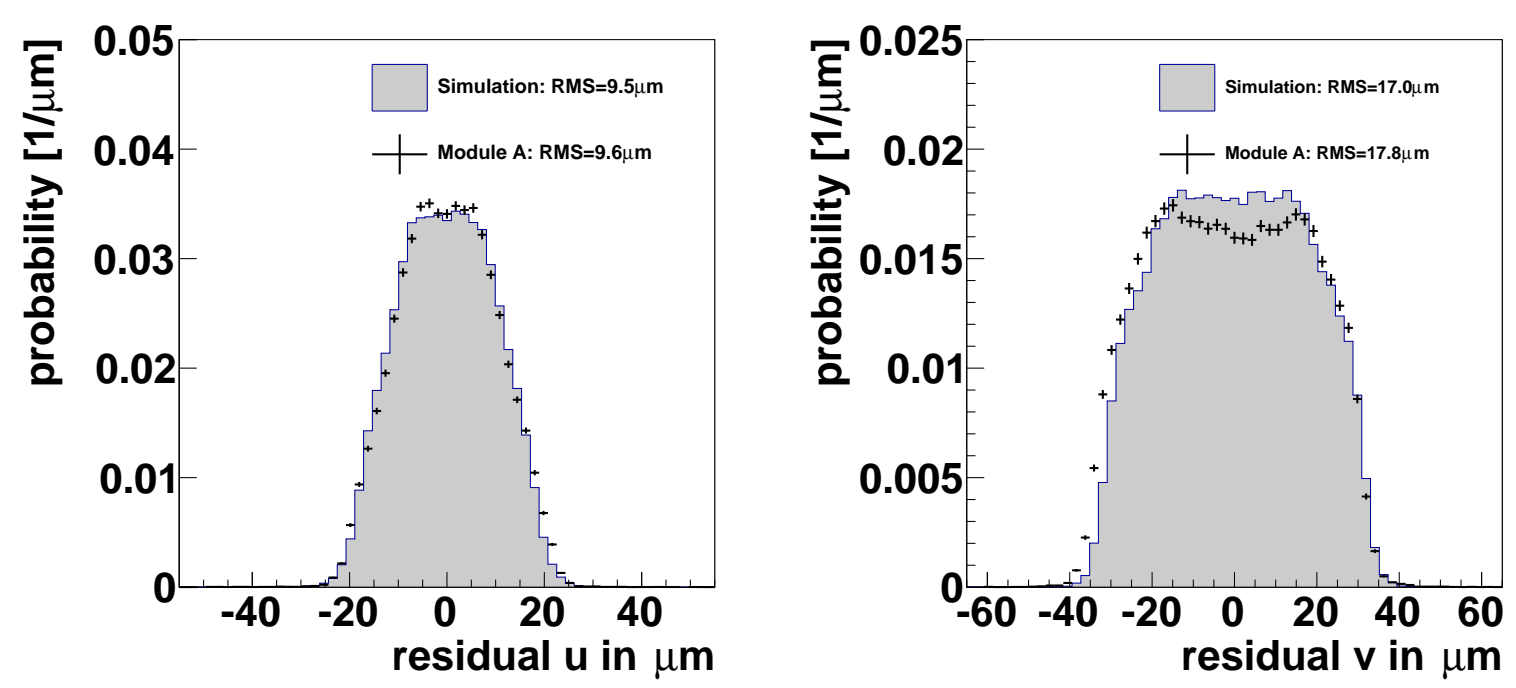

Figure 5.10.: Measured residual distribution for the case of $120 \mathrm{GeV}$ pions at perpendicular incidence for Module A. The width of the residuals is $9.6 \mu \mathrm{m}$ (RMS99) and $17.8 \mu \mathrm{m}$ (RMS99). The simulated detector resolution function is smeared with a Gaussian error in order to simulate the estimated telescope pointing resolution of $1.5 \mu \mathrm{m}$.

allows to estimate the spatial resolution after subtracting the pointing resolution of the telescope. The method to infer the spatial resolution from track residuals was introduced and tested in section 4.4.2.1. The key point is an accurate estimation of the pointing resolution of the telescope using a Kalman Filter based track fitting which takes into account multiple scattering and telescope alignment corrections. In the case of the detector simulation, the distribution of position measurement errors can be obtained directly by comparing the center of gravity of the cluster to the true intersection coordinates of the particle as described in section 3.4 .

The residual distribution for the measurements of the intersection coordinates perpendicular to and along the columns are shown in Fig. 5.10. The residual distribution is obtained for Module A during a test beam at CERN with $120 \mathrm{GeV}$ pions at perpendicular incidence. The pointing resolution of the EUDET telescope estimated by the track fitter is $1.5 \mu \mathrm{m}$. This is significantly better than the expected intrinsic spatial resolution of the DEPFET module. The measured residuals are compared to the simulated distribution of true position measurement errors smeared with the estimated telescope pointing resolution of $1.5 \mu \mathrm{m}$. The detector simulation describes the shape of the residuals well in the $u$ direction. The width of the residuals is taken as the root mean square of the central $99 \%$ of data points (RMS99). The width of the residuals in the $u$ direction is $9.6 \mu \mathrm{m}$ for Module A and $9.5 \mu \mathrm{m}$ for the simulation. However, the measured residual distribution in the $v$ direction shows two shoulders which are not reproduced in the simulation. The appearance of shoulders causes a discrepancy between the width of the residuals from simulation $(17.0 \mu \mathrm{m})$ and test beam data $(17.7 \mu \mathrm{m})$. The origin of these shoulders is still under investigation.

In Fig. 5.11 (left) the effect of the non-perpendicular incident particles on the cluster size is shown. The average cluster size grows and the spatial resolution improves while rotating the DEPFET module to tilt angles $\theta$ ranging from $0^{\circ}$ to $46^{\circ}$. The data is obtained from a test beam at DESY with $3 \mathrm{GeV}$ electrons and agrees well with the results from the detector simulation. The intrinsic spatial resolution 

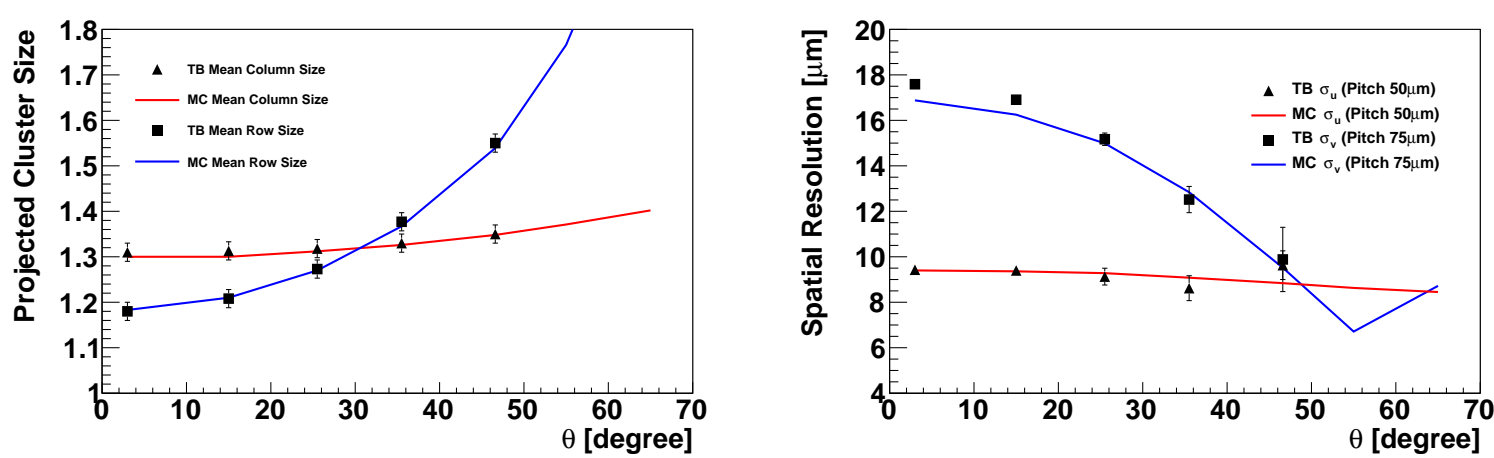

Figure 5.11.: Mean cluster size (left) as a function of the incident angle $\theta=\arctan t_{v}$ of the incoming particle. The incident angle in the other direction $\arctan t_{u}$ is close to zero. The mean number of rows in a cluster increases from 1.2 to 1.6 for the largest measured incident angle of $46^{\circ}$. The positive effect of having larger clusters is visible on the spatial resolution (right). The resolution $\sigma_{v}$ in the $v$ direction is obtained after quadratic subtraction of the telescope pointing resolution and drops from $17.7 \mu \mathrm{m}$ to $9.9 \mu \mathrm{m}$ for $46^{\circ}$.

of Module A with $50 \times 75 \mu \mathrm{m}^{2}$ pixels is measured to be $9.6 \mu \mathrm{m}(17.8 \mu \mathrm{m})$ in the short (long) pixel direction in the case of perpendicular incidence. The simulation shows that a minimum resolution of $6.7 \mu \mathrm{m}$ could be achieved in the long pitch direction for even larger tilts of $55^{\circ}$. At this rotation angle, the average number of hit rows in clusters is 1.8 and the center of gravity is able to interpolate an improved hit position from the signals of the two rows. At even larger angles, the cluster size quickly increases and the signal interpolation is less beneficial. In the $u$ direction, the mild increase in the mean number of hit columns is a secondary effect caused by the overall increase of signal charge in clusters. The quoted error on the measured spatial resolution quickly grows with the tilt angle. The origin of this effect is the increased spacing between the two arms of the EUDET telescope which is needed to accommodate the tilted DEPFET module in the center of the telescope. The pointing resolution at the central DEPFET module depends on the spacing between the arms and degrades from $5.7 \mu \mathrm{m}$ at perpendicular incidence to $16.4 \mu \mathrm{m}$ for a rotation angle of $46^{\circ}$. The error on the measured spatial resolution is dominated by the assumption of an $5 \%$ uncertainty on the estimated telescope pointing resolution.

The spatial resolution of the DEPFET modules operated in Belle II depends on the incidence angle of tracks, the pitch of pixels in the $u$ and $v$ direction and the sensor thickness. For this reason, the spatial resolution reported here can not be applied immediately in the Belle II environment. The detector simulation provides a good model for the data obtained from test beams. Moreover, it contains enough detector physics to extrapolate the estimated spatial resolution from the test beam environment to the Belle II case.

\subsubsection{Study of hit detection efficiency}

Test beams offer the possibility to measure the hit detection efficiency $\epsilon$ of DEPFET modules using a tag and probe technique. The approach followed here is to reconstruct tracks in the telescope requiring hits on the six Mimosa26 sensors only (tag). Then, we can probe whether we find a hit on the DEPFET sensor from the same particle close to the predicted impact point on the device under test. A complication of the efficiency measurement is the rather long integration time of the Mimosa26 planes (114 $\mu$ s) compared 

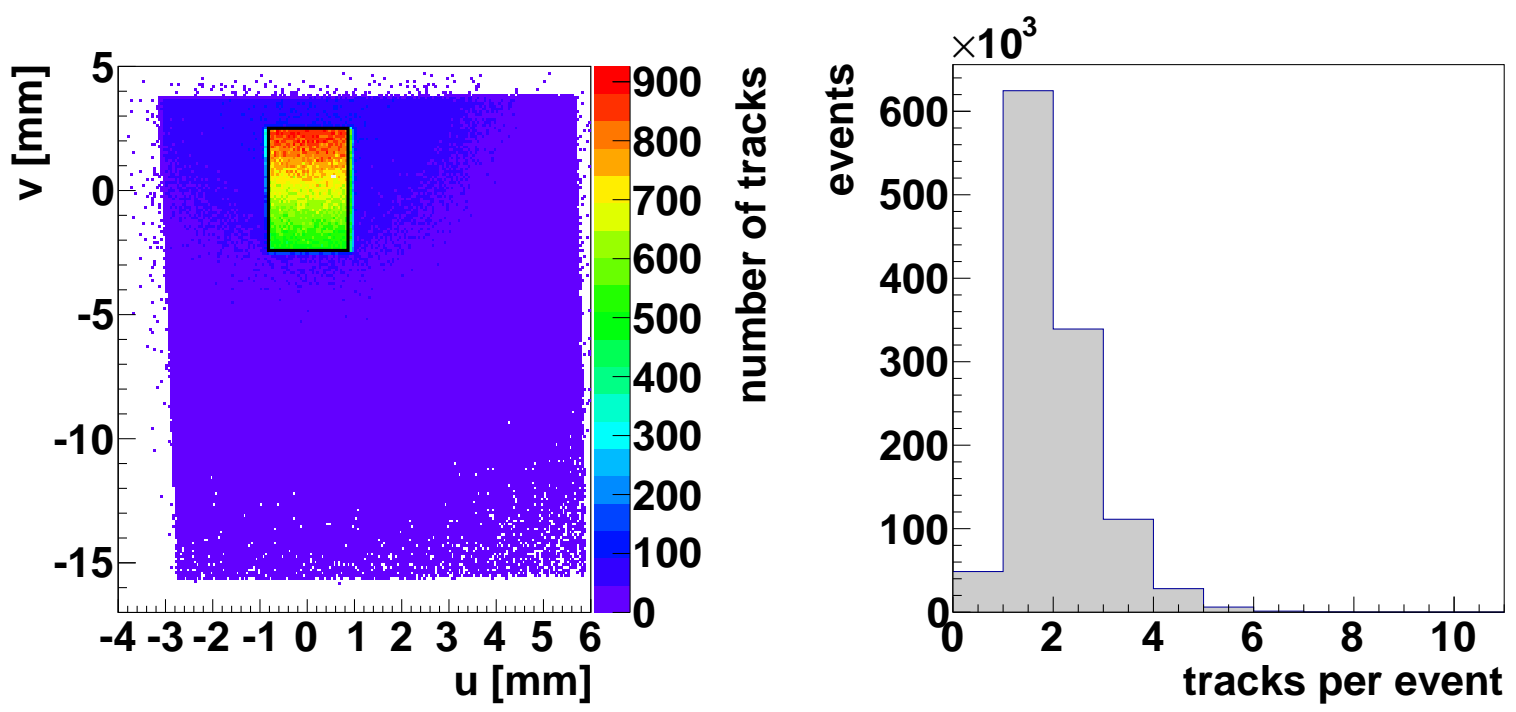

Figure 5.12.: Left: Number of telescope tracks with at least 5 hits in the six Mimosa26 sensors shown in the local $u, v$ coordinates of the tested DEPFET module (B). The size of the trigger area was adjusted to the sensitive area of the tested sensor (black rectangle). The density of primary tracks peaks at the position of the tested sensor while secondary tracks are scattered over the entire acceptance of the telescope. Right: Distribution of the number of telescope tracks per event. Only events with a single track in the entire telescope are used for the efficiency measurement.

to the much shorter integration time of the DEPFET module $(4.8 \mu \mathrm{s})$. Only the first track which triggers the readout is guaranteed to be in the integration time of both the Mimosa26 planes and the DEPFET module. Secondary tracks crossing the telescope within the integration time of the Mimosa26 planes but more than $4.8 \mu$ s after the trigger cannot be detected on the DEPFET plane but will be reconstructed in the telescope. In other words, secondary tracks hitting the sensitive area of the DEPFET module bias the measured hit efficiency $\epsilon$ downwards. The approach followed here is to suppress the effect of secondary tracks by vetoing all events with more than one track in the telescope acceptance 3 .

The efficiency measurements were performed for Module B from a sample of $120 \mathrm{GeV}$ pion tracks measured at CERN, see Fig.5.12. The track reconstruction requires five hits on Mimosa26 planes with a track $\chi^{2}$ value below 100 in the fully aligned telescope. The number of tracks per trigger hitting the small active area of Module B was significantly enhanced by integrating a further trigger plane based on the FE-I4 chip into the TLU as described in Obermann [77]. The main advantage of the new trigger plane is the possibility to trigger on particle tracks traversing an adjustable trigger area which can be positioned around the active sensor area of the device under test. A total number of 0.6 million tracks was available for measurements of the hit detection efficiency in single track events. A hit on the device under test was matched to the particle track if the track residuals were below $100 \mu \mathrm{m}$ in the $u$ and $v$ directions.

Fig. 5.13 shows the two dimensional map of efficiencies for all pixels on the sensor. The statistical errors for a pixel by pixel map are in the order of $1 \%$ limited by the rather small number of 300 tracks

\footnotetext{
${ }^{3}$ The optimal solution to this problem would be the installation of another (reference) DEPFET module in order to tag the first track in an event. However, the available DAQ system was limited to operate a single DEPFET module in the test beam.
} 


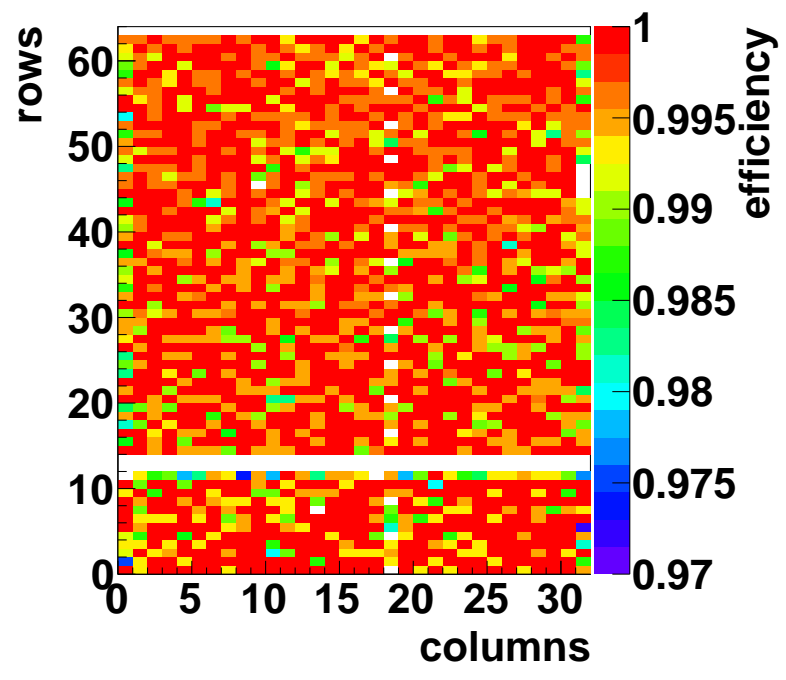

Figure 5.13.: Measured efficiency $\epsilon$ for all $32 \times 64$ pixels of Module B. The average number of tracks per pixel used for the measurement is 300 and results in a statistical uncertainty of $\sim 1 \%$. A total of four rows and parts of one column are masked (white pixels). Apart from masked pixels the measured efficiency is uniform over the sensor area within the statistical errors.

per pixel. Despite the limited statistical accuracy, the two dimensional map demonstrates that only few pixels were masked and shows a uniform hit efficiency over the whole sensor area. A statistically more accurate measurement of the hit efficiency is given Fig. 5.14 where hits in the same pixel column or row are accumulated. The measured hit detection efficiency is better than $99.5 \%$ for all working pixel columns and rows. The efficiency drop in the outermost two columns to $99.2 \%$ is still under investigation. The expected hit detection efficiency from the detector simulation is $99.7 \%$ and agrees well with the experimental result.

In Fig. 5.15 the detection efficiency for pions under perpendicular incidence is shown as a function of the threshold on the signal in the seed pixel (the pixel with largest signal in the cluster). The minimum value of the seed threshold is given by the smallest usable zero suppression threshold. The zero suppression threshold used here is 3 LSB or 525 electrons (Module B). The hit detection efficiency is measured
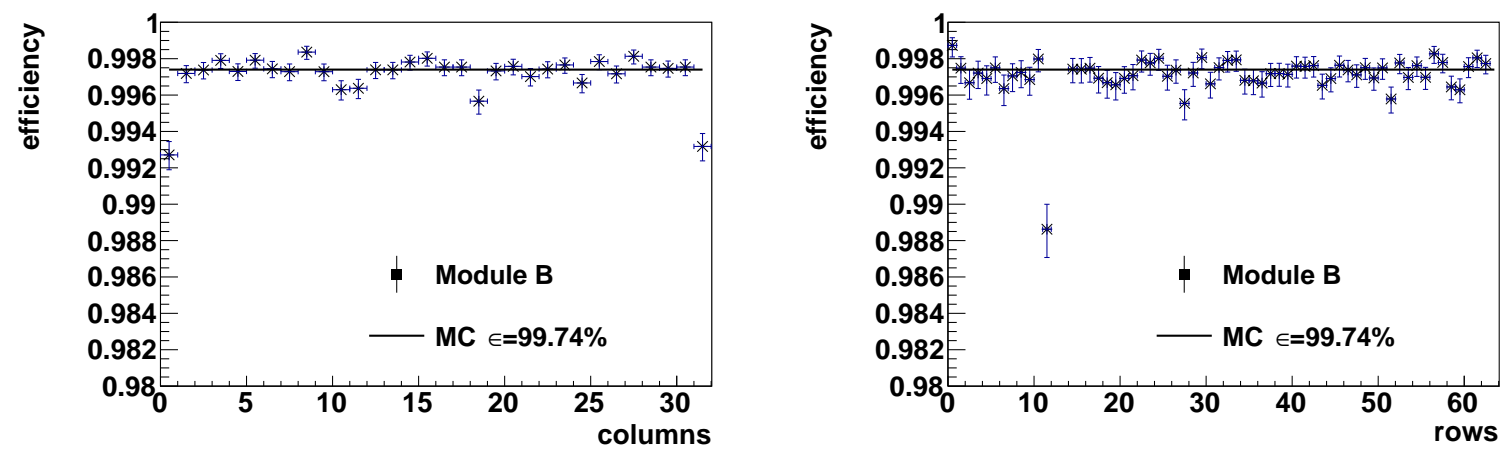

Figure 5.14.: Measured efficiency $\epsilon$ for Module B averaged over columns (left) and rows (right). Apart from columns (rows) with masked pixels, the efficiency is $>99.5 \%$ in the whole sensor area. The efficiency drop to $99.2 \%$ in the outermost is still under investigation. 

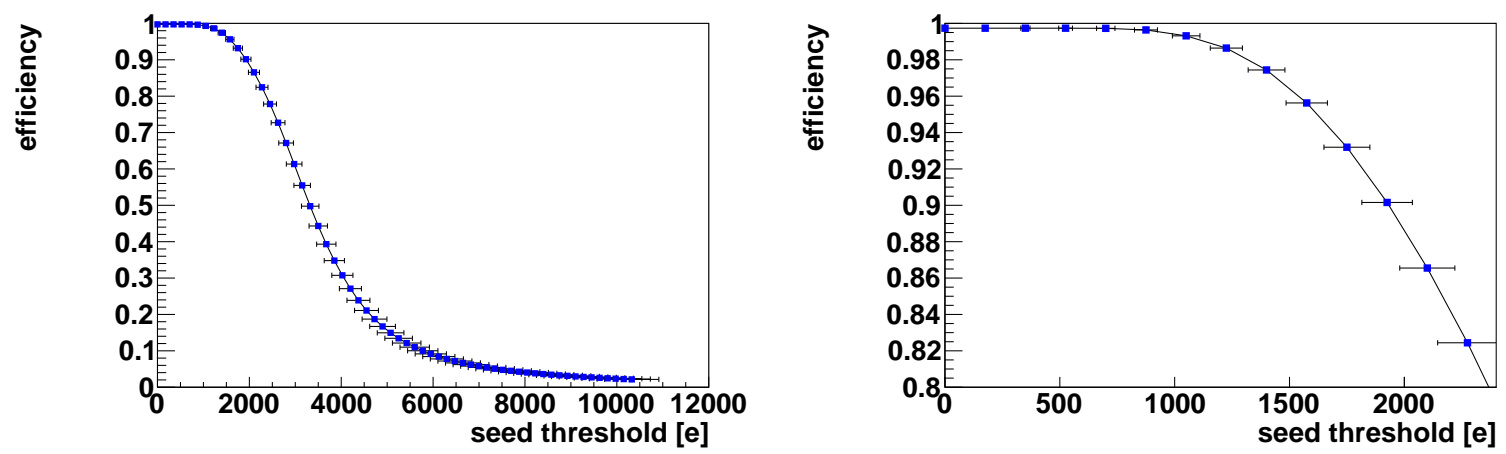

Figure 5.15.: Measured efficiency $\epsilon$ for Module B averaged over the whole sensor area for different thresholds on the seed signal (left). The minimum usable seed threshold was 3 LSB or 525 electrons limited by the smallest usable zero suppression threshold. The measured efficiency is $>99.5 \%$ for a seed threshold of 525 electrons but falls below $99 \%$ for thresholds larger than 1200 electrons (right).

for seed thresholds from 525 electrons to roughly 12000 electrons. The statistical error on the efficiency is $<0.1 \%$ for all seed thresholds. The main experimental error is the calibration of the least significant bit in terms of an equivalent number of electrons. The most important observation is the steep decrease of the hit efficiency for thresholds larger than 1200 electrons. The origin of this effect will be discussed in more detail in the next section.

\subsubsection{Study of in-pixel charge collection}

The precise pointing resolution of the EUDET telescope offers the possibility to carry out in-pixel scans of hit detection efficiency, charge collection and charge sharing properties of the thinned DEPFET sensors. Fig.5.16 introduces the layout of the Belle II specific fourfold pixel cell and defines the in-pixel coordinates $u_{p}, v_{p}$ used for these studies. The fourfold pixel cell consists of a pair of double pixels with a shared clear implantation. The fourfold pixel is the smallest periodic unit on the sensor.

The in-pixel maps are all computed for Module A using a sample of $\sim 1$ million $120 \mathrm{GeV}$ pions hitting the sensor at perpendicular incidence. The binning for the two dimensional maps of the mean seed signal and cluster size is $30 \times 40$ leading to an average number of 100 track per bin. The resulting bin size is $3.3 \times 3.8 \mu \mathrm{m}^{2}$ can still be be resolved by precisely reconstructed telescope tracks having hits on all six Mimosa26 planes. For the two dimensional maps of the hit efficiency and the cluster charge the binning is chosen to be $10 \times 20$ and the bin size is $10 \times 7.5 \mu \mathrm{m}^{2}$. The different binning is motivated by the larger number of tracks per bin needed to estimate efficiencies and small changes of the mean cluster charge in the fourfold pixel cell.

Fig. 5.17 shows the mean cluster charge as a function of the in-pixel hit position. Both data from the prototype module and the detector simulation show a decrease of the mean cluster charge at the position of the single pixel corners in the fourfold pixel cell. The mean cluster charge drops from 4200 electrons in the pixel center to 3600 electrons in the corners. The charge loss in pixel corners is reproduced by the simulation. The detector simulation works on the assumption that all signal electrons created in the sensor volume are collected in internal gates. The only remaining mechanism for charge loss in the detector simulation is the zero suppression threshold of 600 electrons. For particles hitting in between 


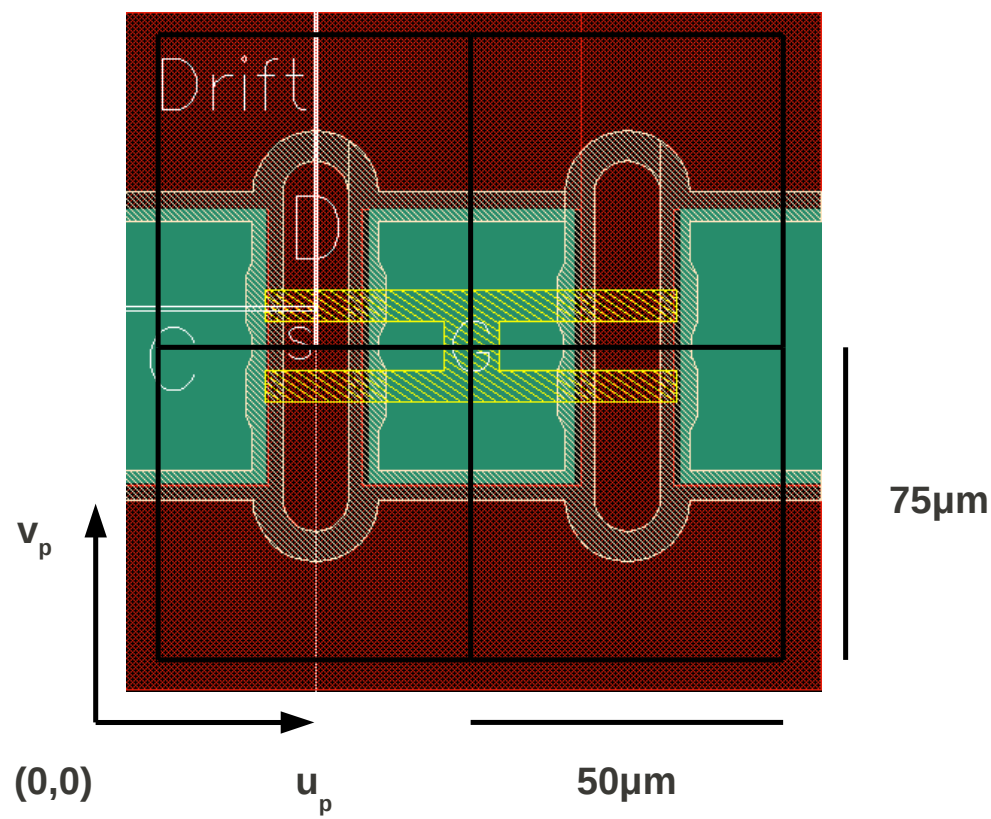

Figure 5.16.: DEPFET sensor layout for Belle II with two double pixels having shared source and clear implantations. The coloured regions are Clear ('C', green), Drift ('DRIFT', red), Drain ('D', red), Source ('S', red) and Gate contact ('G', yellow). The four internal gates are implanted below the gate contacts between the Drain and Source regions. The figure defines the local pixel coordinates $u_{p}$ and $v_{p}$ used for all subsequent in-pixel studies. The origin of the pixel coordinates is the lower left corner of the four fold pixel. 

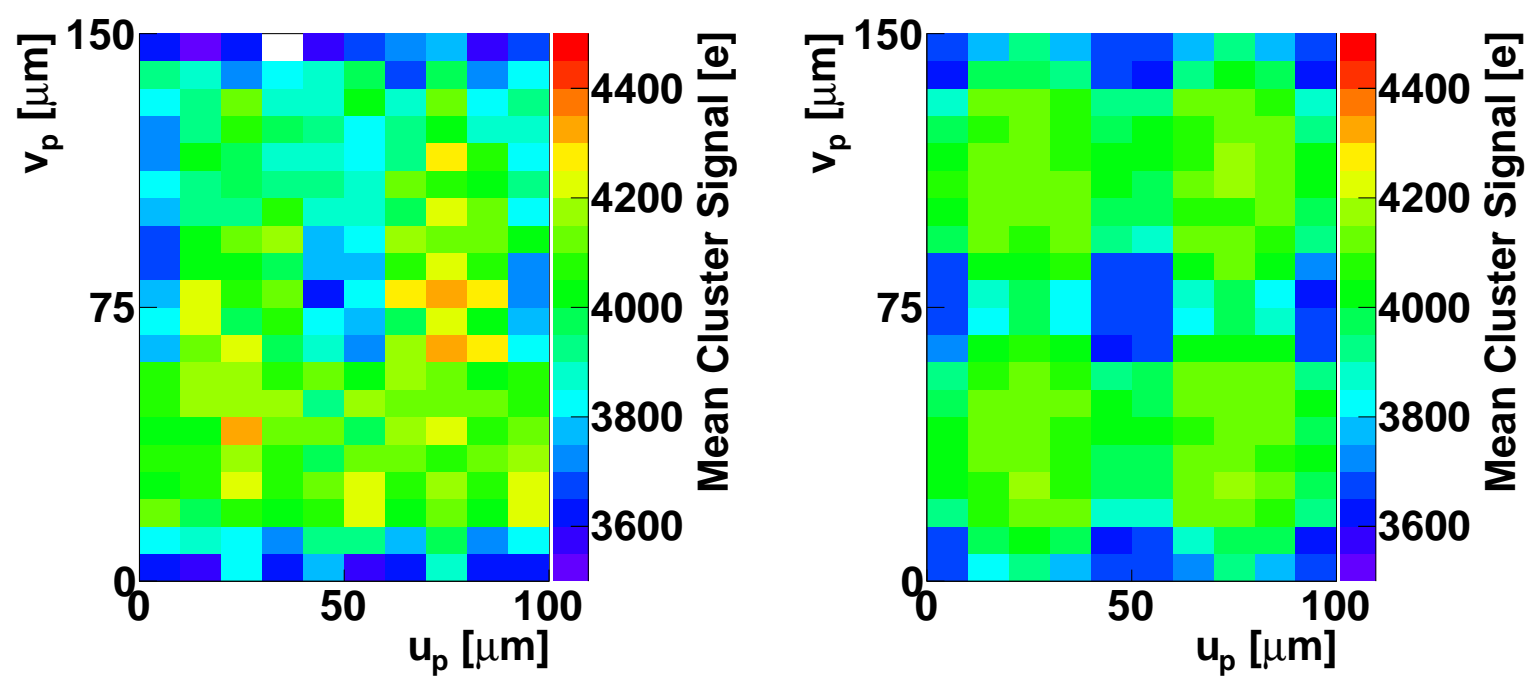

Figure 5.17.: Map of the mean cluster signal from Module A (left) and the DEPFET detector simulation (right) as a function of the predicted hit position in the four fold pixel cell. A reduction of the mean cluster charge is observed in the center of the clear and drift regions in the corners of the four single pixel cells $\left(50 \times 75 \mu \mathrm{m}^{2}\right)$. The mean cluster charge drops from 4200 electrons in the pixel center to 3600 electrons in the corners. The charge loss in pixel corners is reproduced by the simulation.

two pixels, charge sharing and energy loss fluctuations allows the detected signal in neighbour pixels to fall below the zero suppression threshold. This signal charge collected in neighbour pixels is lost after zero suppression.

The impact of the observed charge loss on the hit detection efficiency is studied in Fig.5.18 for two different seed threshold of 3 LSB (525 electrons) and 6 LSB (1050 electrons). Even for the low threshold case, the hit detection efficiency drops to $97 \%$ near the corner of the fourfold pixel. The hit detection inefficiency grows significantly as the seed threshold is raised to 1050 electrons. The inefficiency increases in magnitude and grows from the corners to the center of the pixel area. This data confirms the hypothesis that energy loss fluctuations in thin silicon and charge sharing between neighbouring pixels are responsible for the observed charge losses and hit detection inefficiency.

\subsubsection{Study of in-pixel charge sharing}

Charge sharing means that the primary ionization charge is collected in the internal gates of more than one pixel. In case this shared charge exceeds the zero suppression threshold, a multi-pixel cluster is detected. Multi-pixel cluster improve the spatial resolution as demonstrated in the previous section. On the other hand, charge sharing can potentially lead to charge loss if the shared charge falls below the zero suppression threshold. The detector simulation implements a detailed model for the diffusion of signal charge in thin DEPFET sensors. The purpose of this section is to test the diffusion model using position resolved maps of the cluster size and the seed signal.

Fig. 5.19 shows the mean cluster size as a function of the in-pixel hit position determined from track extrapolation. In order to allow a quantitative comparison between real data and simulation, the profiles of the mean cluster size along the $u_{p}$ and $v_{p}$ directions are given in Fig.5.20 The mean cluster size is 

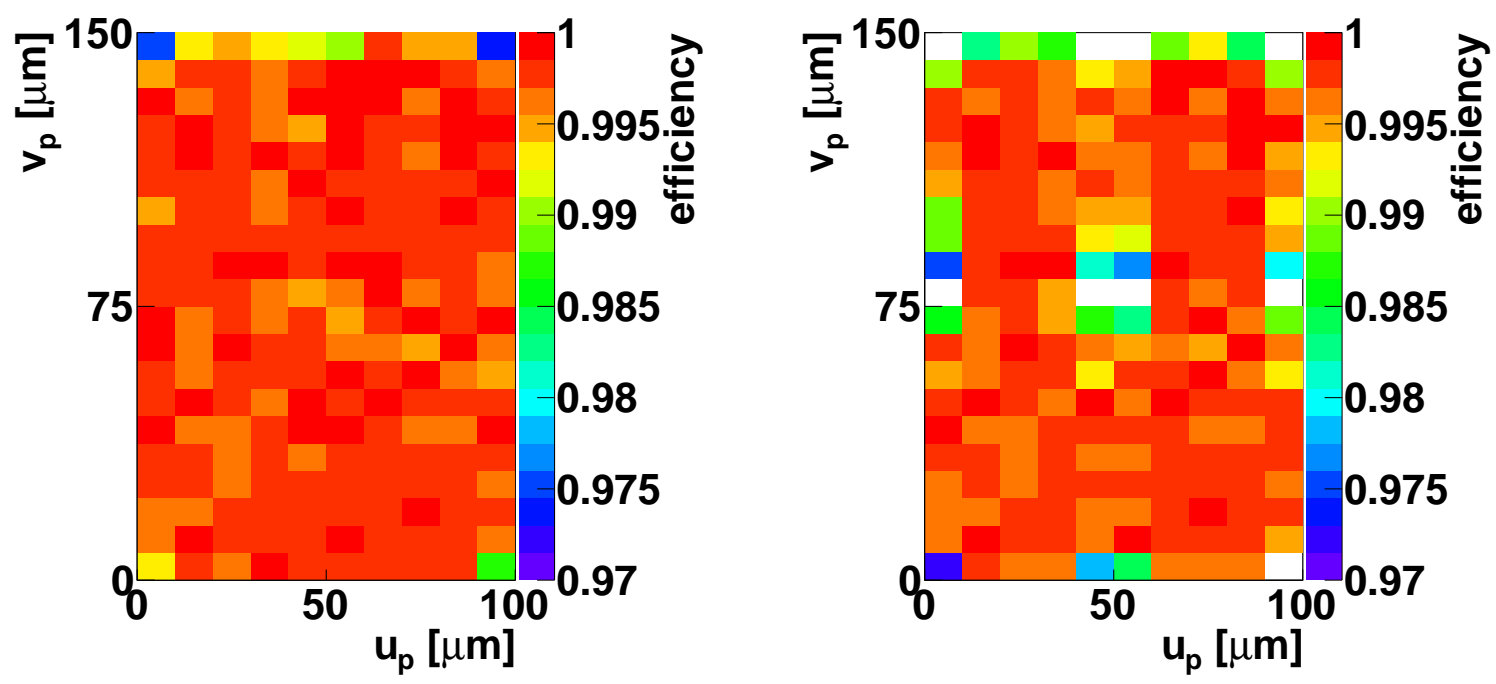

Figure 5.18.: Map of the hit efficiency $\epsilon$ in the four fold pixel cell for a seed threshold of 525 electrons (left) and 1050 electrons (right) in response to $120 \mathrm{GeV}$ pions at perpendicular incidence. Even for a low threshold, a small hit detection inefficiency is visible at the outer corners of the four fold pixel cell. The hit detection inefficiency grows significantly as the seed threshold is raised to 1050 electrons (right). The bin size is $10 \times 7.5 \mu \mathrm{m}^{2}$.

one near the geometrical center of each of the four pixels and two at the pixel edges. The measured data confirms the prediction of border regions with enhanced charge sharing. The mean cluster size in the pixel corners is three and not four as could be expected from geometry. The charge shared to the fourth pixel always falls below the zero suppression threshold and gives an explanation for the observed charge loss in the corners. The most significant deviations between test beam data and simulation are visible in the center of the drift regions of the four fold pixel cell at $v_{p}=0 \mu \mathrm{m}$ and $v_{p}=150 \mu \mathrm{m}$. The one pixel regions appears rounded in these regions due to not modeled details of the electrical potential in the pixels as compared to the simulation. The appearance of shoulders in the measured residuals in the $v$ direction are most likely related to this observation.

Charge sharing between neighbouring pixels implies that the mean seed signal depends on the in-pixel hit positions as shown in Fig.5.21 and Fig.5.22. The mean seed signal is rather flat in the pixel centers where single pixel clusters dominate and decreases towards the pixel edges from about 4500 electrons to 2500 electrons. In the pixel corners, the mean seed signal is as small as 1600 electrons due to large charge sharing. The small seed signal in the pixel corners is the most likely explanation for the observed hit detection inefficiency and requires a sensor operation with small signal thresholds. 

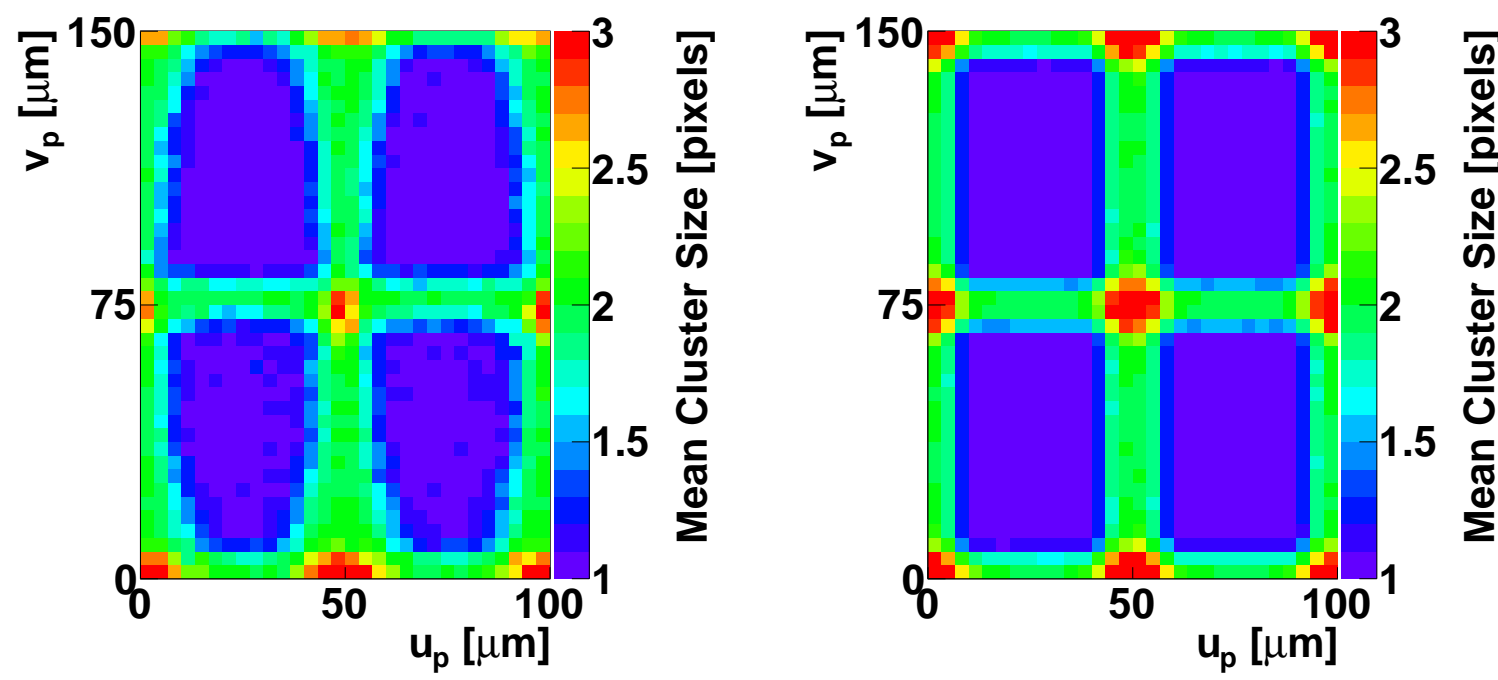

Figure 5.19.: Map of the mean cluster size in the four fold pixel cell for Module A (left) and the detector simulation (right) in response to $120 \mathrm{GeV}$ pions at perpendicular incidence. The average cluster size is one near the geometrical center of the four pixels, two near the pixel edges and approaches three in the pixel corners.
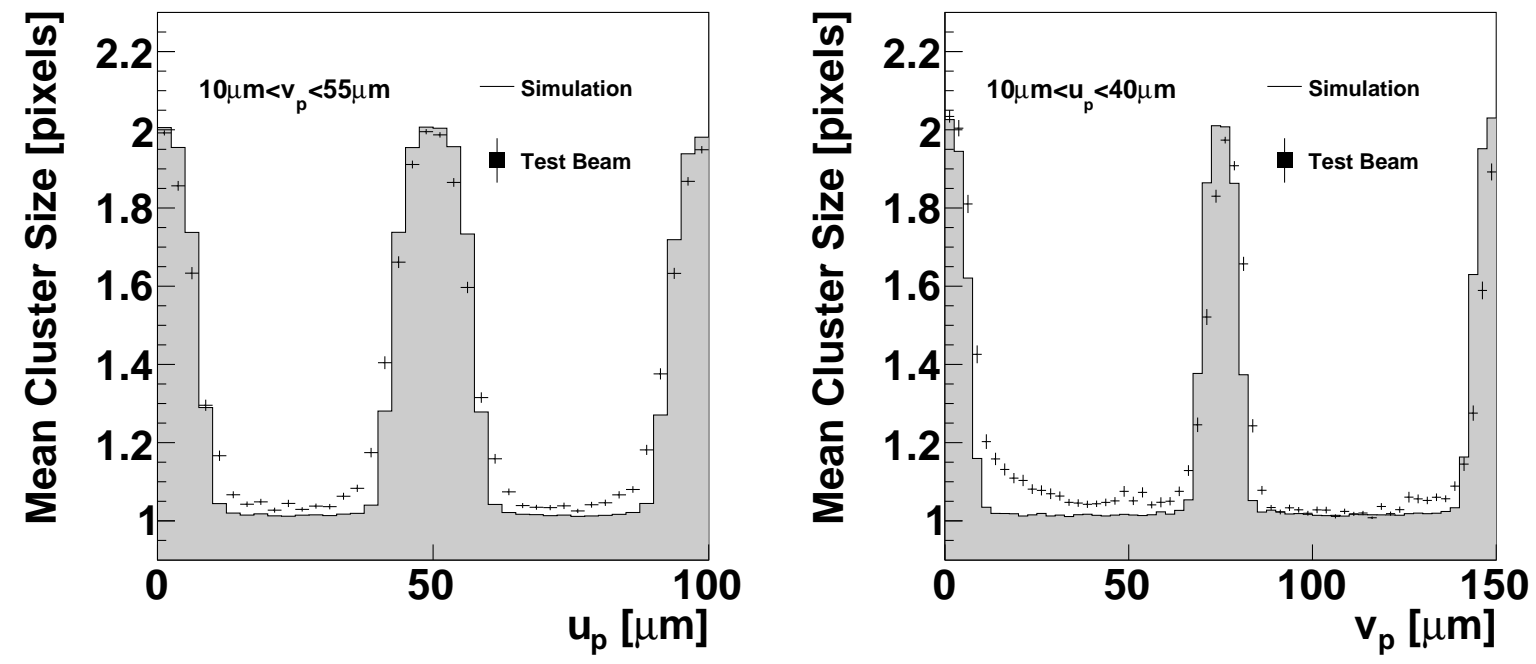

Figure 5.20.: Profile of the mean cluster signal along the $u_{p}$ coordinate (left) and the $v_{p}$ coordinate (right). The simulation agrees well with the real profile in the $u_{p}$ direction and demonstrates the existence of border regions with enhanced charge sharing and increased cluster size. The cluster size profile in the $v_{p}$ directions show an asymmetry between the odd and even pixel cells that is not reproduced in the simulation. 

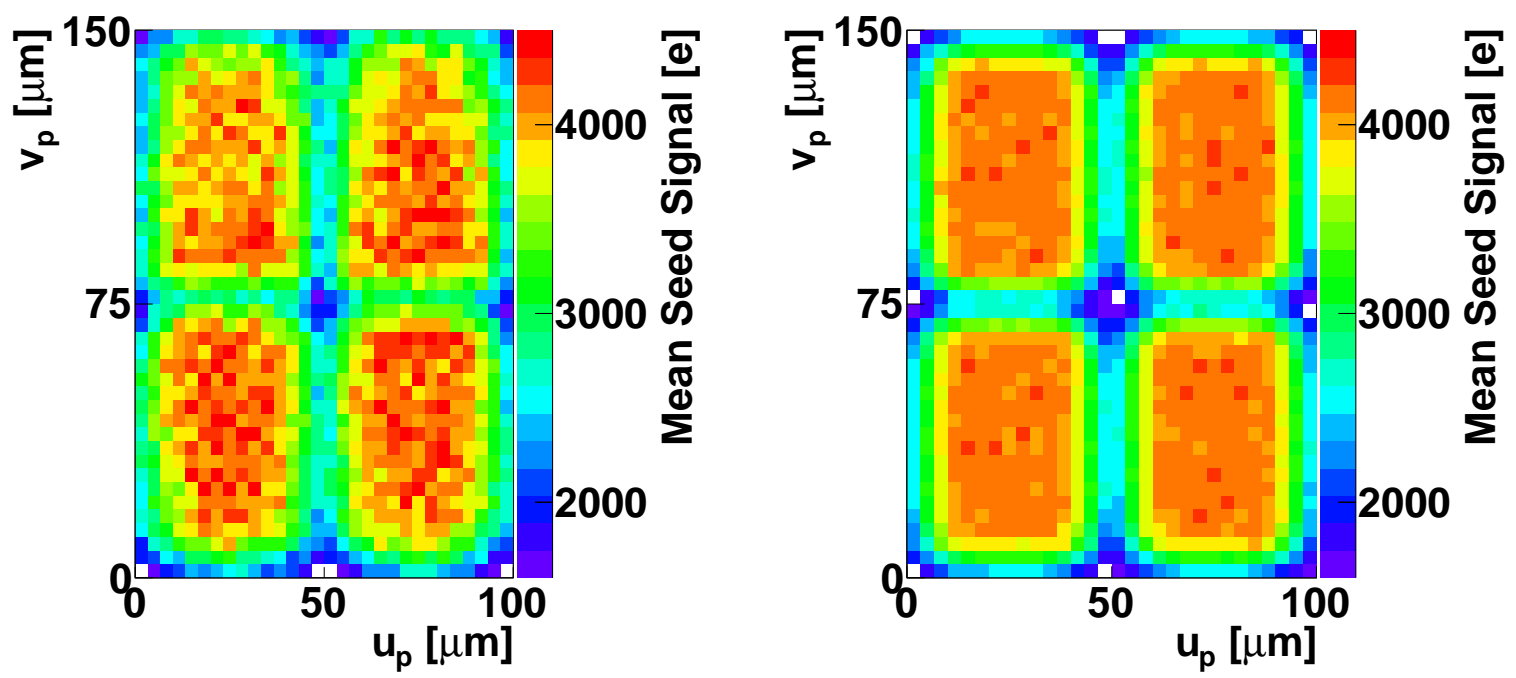

Figure 5.21.: Map of the mean seed signal in the four fold pixel cell for Module A (left) and the detector simulation (right) in response to $120 \mathrm{GeV}$ pions at perpendicular incidence.
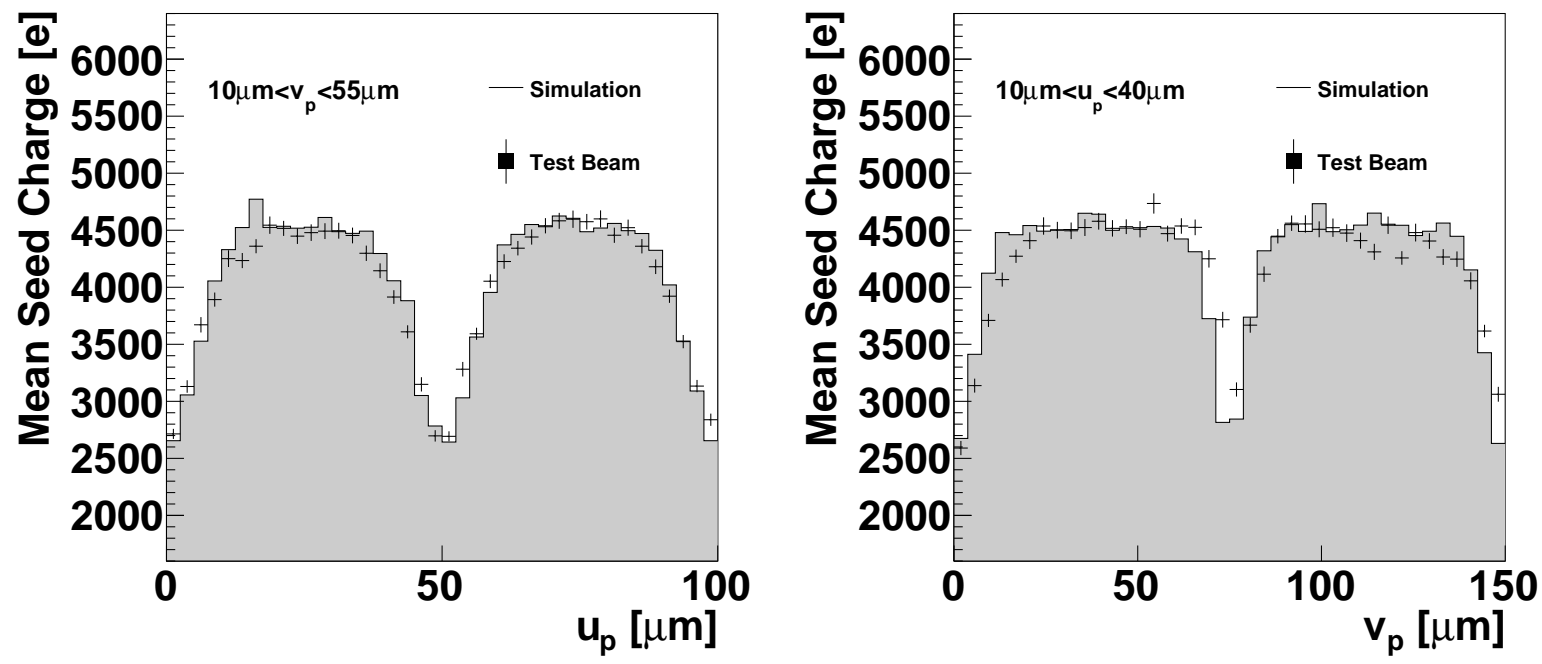

Figure 5.22.: Profile of the mean cluster signal along the $u_{p}$ coordinate (left) and the $v_{p}$ coordinate (right). The simulation agrees well with the real module data in the $u_{p}$ and the $v_{p}$ direction. 


\section{Summary and outlook}

The future Belle II experiment at the SuperKEKB flavour factory requires a vertex detector of unprecedented performance to take advantage of the high beam luminosity and provide an increased vertex resolution. A novel, two layer pixel vertex detector is foreseen for Belle II, based on the DEPFET technology. The Depleted Field Effect Transistor (DEPFET) pixel structure is an advanced semiconductor detector which delivers position sensitive particle detection. The main advantages of this detector concept are low noise, internal signal amplification, small pixels and thinned detectors.

A crucial tool for the optimization of the vertex resolution is a validated algorithm to simulate the response of a DEPFET pixel detector to a charged particle. The detector simulation provides a description of the straggling of particles in silicon, the drift, diffusion and collection of the signal and the response of the read-out electronics. One important contribution of this thesis is the development of a fast model for the signal drift, diffusion and collection in fully depleted DEPFET pixel sensors. This model is derived from a detailed semiconductor device simulation performed at the Semiconductor Laboratory of the Max-Planck Institutes for Physics and for Extraterrestrial Physics (MPI HLL). The model gives an approximate solution for the drift times of signal electrons in the sidewards depleted sensor. Moreover, the model accounts for slow, diffusion-dominated signal collection near the borders of pixels. The model yields a precise prediction of the spatial resolution of the detector, given design parameters such as the pixel size, the sensor thickness and the electronics noise.

The second part of this thesis is devoted to the results of measurements of the response of DEPFET detector prototypes to a beam of charged particles in test beam lines at CERN and DESY. All test beam measurements were performed using the EUDET/AIDA beam telescope and its copies. Substantial effort was devoted to the development of new methods for the calibration, tracking and alignment of the beam telescope, that allow a quantitative determination of the signal distribution, hit efficiency and spatial resolution. These methods confirmed that all six Mimosa26 planes in the EUDET/AIDA telescope achieve an intrinsic spatial resolution of $3.5 \mu \mathrm{m}$ exposed to electron beams in the test beam lines at DESY. For the first time, a position resolved measurement of the radiation length $X / X_{0}$ of DEPFET detectors was performed from reconstructed scattering kinks. This result shows the prospect of routinely measuring the radiation length of position sensitive detectors and their passive components at test beams.

The relevant test beams for the validation of the detector simulation are based on the latest DEPFET sensor production (PXD 6). The sensors are thinned to $50 \mu \mathrm{m}$ and feature pixel cell sizes of $50 \times 75 \mu \mathrm{m}^{2}$ specifically designed for Belle II. The readout of the prototype detectors is based on the first production version of the Drain Current Digitizer for Belle II (DCDB) front-end chip. The availability of prototypes with Belle II specific sensors and readout chips allows a direct comparison between the intrinsic spatial resolution predicted for Belle II and measurements at test beams.

The best achievable intrinsic spatial resolution of DEPFET pixel detectors has far reaching consequences for the vertex resolution of Belle II. The intrinsic spatial resolution of the pixel detector strongly depends on the pixel pitch and the incidence angle of tracks hitting the sensor. In the sensor direction 


\section{Summary and outlook}

parallel to the pixel columns ( $50 \mu \mathrm{m}$ pitch), the measured resolution is $9.6 \mu \mathrm{m}$ for tracks hitting the sensor at normal incidence. In the sensor direction perpendicular to the columns ( $75 \mu \mathrm{m}$ pitch), the measured resolution is $17.8 \mu \mathrm{m}$ for tracks at normal incidence. However, the resolution improves for larger incidence angles and reaches a minimum of $6.7 \mu \mathrm{m}$ at an incidence angle of $55^{\circ}$ relative to the sensor normal. The agreement between measurement and simulation is better than $10 \%$. A long pitch of $75 \mu \mathrm{m}$ allows to keep the spatial resolution well below $10 \mu \mathrm{m}$ in the forward-backward part of the vertex detector while a pitch of $50 \mu \mathrm{m}$ is acceptable near the interaction point. The proposed design of the pixel vertex detector for Belle II features a variable pixel pitch along the beam pipe to maintain an optimal resolution over the entire tracking acceptance.

The hit detection efficiency of the prototype detectors is better than $99.5 \%$ requiring a signal of at least 600 electrons per pixel. The efficiency strongly depends on the signal threshold and falls below $98 \%$ for thresholds above 1200 electrons. At perpendicular incidence of tracks on the sensor, the cluster size and seed signal depend on the diffusion of signal electrons in the pixel cell. For this reason, the in-pixel resolved measurements of the mean cluster size allow to test the drift and diffusion model of the detector simulation. As expected from the detector simulation, the cluster size is one in the center of pixels and increases towards the pixel borders where signal collection is dominated by diffusion.

The detector simulation algorithm discussed in this thesis has been a key element in the optimization of the baseline design of the pixel vertex detector for Belle II. The good agreement with recent test beam data gives us confidence that the most important factors affecting the pixel performance are modeled accurately and that the baseline design will be an excellent tool to achieve the physics goals of Belle II.

Final design DEPFET ladders for Belle II will become available for testing in spring 2015. The set of test beam measurements presented in this thesis should be repeated to confirm the scalability of the results from small scale prototypes to full-size detectors. Moreover, the effect of the Lorentz force in a 1.5 T magnetic field at Belle II on charge collection in DEPFET sensors should be measured in upcoming test beams. 


\section{A. Track fitting in the EUDET telescope with Kalman Filters}

In this appendix, we give the mathematical details on the application of Kalman Filters for track fitting in the EUDET beam telescope. After introducing a dynamic system model, or track model, for high momentum charged particles in the telescope, we describe how the Kalman Filter technique can be applied to estimate local track parameters at all sensors in the telescope. In particular, this appendix addresses a number of specific adjustments to the general Kalman Filter formalism used for track fitting in this thesis:

- The track model is nonlinear in case the telescope contains tilted sensors. In order to apply Kalman Filters, a linearization of the track model is needed. The main idea followed here is to linearize the track model around the axis of the particle beam itself. In this case, the Kalman Filter is applied to a linearized system and filters the deviations of the track parameters from the beam axis.

- The EUDET telescope requires track fitters which attain their highest precision at the device under test placed between the telescope arms. For that purpose different types of Kalman Filters are combined. For track fitting, the local track parameters are computed as a weighted means of a forward and backward filter. For the material estimation presented in section 4.4.2.3 a further time reversed filter is needed.

- In order to initialize the forward filter, we use the collimated beam model developed in section 4.4.2.2. This is especially useful to constrain telescope shearing and torsion during telescope alignment.

In order to increase the numerical robustness of Kalman Filters all Kalman Filters used in this thesis are implemented as Square Root Information Filters as described in Kaminski [78]. The final section of this appendix gives the results of pseudo experiments to validate the developed track fitter.

\section{A.1. A track model for the EUDET beam telescope}

A dynamic system model for the passage of a charged particle through the EUDET telescope is a prerequisite for the application of Kalman Filters to track fitting [45]. The dynamic system model, or track model, developed for track fitting in the EUDET telescope consists of four building blocks:

- A stochastic model for the particle beam: The purpose is to provide a realistic model of the distribution of track parameters at a reference plane placed just before the first telescope sensor.

- A deterministic vacuum propagator function to map track parameters from sensor to sensor. Track parameters are transported along $3 D$ straight lines between sensor planes. 
- A stochastic model for multiple scattering: A local scattering function maps track parameters before scattering to track parameters after scattering. Multiple scattering at thin sensors amounts to small randomized changes of the incident angles at the sensor plane.

- A stochastic model to simulate hits on sensor planes: The purpose is to model the relation between the true track parameters on the one hand side and measured hit coordinates on the other.

The model developed here allows to simulate the passage of a particle through the telescope as a discrete stochastic process. Starting from the creation of a beam particle at the $z=0$ plane, the simulation follows the particle in a sequence of $3 D$ straight line steps from sensor to sensor. Multiple scattering at sensor planes is taken into account as a small change to the local incident angle of the particle that will be used for stepping to the next sensor. In order to account for scattering in the air between sensors, further scattering planes are simulated at half flight length between sensors.

\section{A Gaussian model for the particle beam}

Particles entering the beam telescope originate from a collimated particle beam. We will assume a monochromatic particle beam where all particles have the same momentum $p$, mass $m$ and electric charge $q^{1}$ The distribution of local track parameters

$$
\lambda_{b}=\left(\begin{array}{c}
t_{x} \\
t_{y} \\
x \\
y
\end{array}\right)
$$

at the $z=0$ plane is modeled as a $4 D$ Gaussian distribution. Following the parametrization of real particle beams developed before (see section 4.4.2.2, , the mean value for track parameter is $\left\langle\lambda_{b}\right\rangle=0$ while the covariance is $C_{b}$.

$$
C_{b}=\left(\begin{array}{cccc}
\sigma_{t_{x}, t_{x}}^{2} & 0 & \sigma_{t_{x}, x}^{2} & 0 \\
0 & \sigma_{t_{y}, t_{y}}^{2} & 0 & \sigma_{t_{y}, y}^{2} \\
\sigma_{t_{y}, y}^{2} & 0 & \sigma_{x, x}^{2} & 0 \\
0 & \sigma_{t_{y}, y}^{2} & 0 & \sigma_{y, y}^{2}
\end{array}\right)
$$

\section{Local track parameters and vacuum propagation}

Without a magnetic field and in a vacuum, the flight path of a particle is a $3 D$ straight line. For a given local coordinate system, we can parametrize the state of the particle at the $w=0$ plane by the following four variables

$$
\lambda=\left(t_{u}, t_{v}, u, v\right)^{T}
$$

where $t_{u}=d u / d w$ and $t_{v}=d v / d w$ are the local directions tangents and $u, v$ are the intersection coordinates with the $w=0$ plane. The flight path of the particle in vacuum is given as

\footnotetext{
${ }^{1}$ The momentum resolution of a real particle beam at DESY or at CERN SPS H6 is around 1\%. For a real beam test, contamination of the beam with other particle species and a smearing of the particle momentum should be considered as systematic uncertainties.
} 


$$
\vec{q}(s)=\vec{a}+s \cdot \frac{\vec{b}}{|\vec{b}|}
$$

where $\vec{a}=(u, v, 0)$ is a $3 D$ point on the particle's path and $\vec{b}=(\tan \theta, \tan \psi, 1)$ denotes the direction vector and $s$ is the path length. The $3 D$ straight line can be parametrized relative to any other local coordinate system. The vacuum track propagator

$$
\lambda_{k}=f_{k \mid l}\left(\lambda_{l}\right)
$$

maps the track parameters $\lambda_{l}$ at the plane $w_{l}=0$ to track parameters $\lambda_{k}$ relative to the $w_{k}=0$ plane of another local coordinate system. The track propagator $f_{k \mid l}$ is known analytically for any pair of coordinate systems, see for example Karimäki [64]. In order to compute the track propagator, the coordinate transformation from $l$ coordinates to $k$ coordinates is needed. Using the notations developed in section 3.2.3, this coordinate transformation can be written as

$$
\vec{q}_{k}=R_{k \mid l}\left(\vec{q}_{l}-\vec{r}_{k \mid l}\right)
$$

where $\vec{r}_{k \mid l}=R_{l}\left(\vec{r}_{k}-\vec{r}_{l}\right)$ denotes a sensor shift vector and $R_{k \mid l}=R_{k} R_{l}^{T}$ is a sensor rotation matrix for sensor $k$ in the coordinate system of sensor $l$. Then, the intersection coordinates of a $3 D$ line with the $w_{k}=0$ plane are denoted as $u_{k}$ and $v_{k}$ and can be computed by the following formulas.

$$
\begin{aligned}
& u_{k}=\left(\overrightarrow{a_{l}}-\vec{r}_{k \mid l} \frac{\left(\vec{r}_{k \mid l}-\overrightarrow{a_{l}}\right) \cdot R_{k \mid l} \vec{w}}{\vec{b} \cdot R_{i} \vec{w}} \overrightarrow{b_{l}}\right) \hat{u} \\
& v_{k}=\left(\overrightarrow{a_{l}}-\vec{r}_{k \mid l} \frac{\left.\left(\vec{r}_{k \mid l}-\vec{a}_{l}\right) \cdot R_{k \mid l} \vec{w}_{\vec{b}}\right) \hat{v}}{\vec{b}_{l} \cdot R_{k \mid l} \vec{w}}\right.
\end{aligned}
$$

Here, $\hat{u}=(1,0,0), \hat{v}=(0,1,0)$ and $\hat{w}=(0,0,1)$ are unit vectors in the local coordinate system. The direction vector $\vec{b}_{k}$ of the line relative to the $w_{k}=0$ plane is given by

$$
\vec{b}_{k}=R_{k \mid l} \vec{b}_{l}
$$

and the flight length $S_{k \mid l}$ from sensor $l$ and to sensor $k$ is $S_{k \mid l}$.

$$
S_{k \mid l}=\left|\vec{b}_{l}\right| \frac{\left(\vec{r}_{k \mid l}-\overrightarrow{a_{l}}\right) \cdot R_{k \mid l} \vec{w}}{\overrightarrow{b_{l}} \cdot R_{k \mid l} \vec{w}}
$$

In a real beam telescope, the particle traverses air between the sensors and the radiation length in air is given by $S_{k \mid l} / S_{\mathrm{d}}{ }^{2}$. Finally, the transport matrix $F_{k \mid l}$ is defined as the derivative of the vacuum propagator.

$$
F_{k \mid l}=\frac{\partial f_{k \mid l}}{\partial \lambda_{l}}
$$

\footnotetext{
${ }^{2}$ At room temperature and normal pressure, the value for $S_{0}$ is given by $305000 \mathrm{~mm}$. For a typical flight length in air of $20 \mathrm{~mm}$ between sensors, the radiation length $S / S_{0}=0.005 \%$ is roughly one tenth of the radiation length of a Mimosa26 module.
} 


\section{Multiple scattering in telescope sensors}

The multiple scattering of the particle at the $k$ th silicon sensor in the telescope can parametrized by two mutually orthogonal, uncorrelated scattering angles $\theta_{1}^{k}$ and $\theta_{2}^{k}$ which are assumed to be small, see Wolin [79]. The $2 \times 2$ covariance matrix $Q_{k}$ for the scattering angles $\theta_{1}$ and $\theta_{2}$ at layer $k$ has the general form

$$
Q_{k}=\left(\begin{array}{cc}
\sigma_{m s}^{2} & 0 \\
0 & \sigma_{m s}^{2}
\end{array}\right)
$$

where $\sigma_{m s}^{2}$ is the variance of the projected multiple scattering angle in the so called comoving frame. The scattering angle of the particle after many scattering with atoms in the sensor roughly follows a Gaussian distribution with a standard deviation of

$$
\sigma_{m s}=\frac{13.6 \mathrm{MeV}}{p c \beta} z \sqrt{\frac{T}{T_{0}}}\left(1+0.038 \log \left[\frac{T}{T_{0}}\right]\right)
$$

where $z$ is the electrical charge in units of $e$ and $T / T_{0}$ is the sensor's radiation length and $p$ is the particles momentum in units of $\mathrm{MeV} / \mathrm{c}$. The so called scatter function $g_{m s}$ computes the track parameters after scattering $\lambda_{k}^{\star}$ from the track parameters before scattering $\lambda_{k}$ and the two scattering kinks $\eta_{k}=$ $\left(\theta_{1}, \theta_{2}\right)$.

$$
\lambda_{k}^{\star}=g_{m s}\left(\lambda_{k}, \eta_{k}\right)
$$

Without a magnetic field, the scatter function and its partial derivatives can be computed analytically, see for example Wolin [79]. For thin sensors, the lateral displacement of the particle due to multiple scattering can be neglected. Therefor, we focus in the computation of the direction tangents $t_{u}^{\star}$ and $t_{v}^{\star}$ after scattering. The key step is the explicit construction of the comoving frame relative to the local coordinates system $u_{k}, v_{k}, w_{k}$ of the sensor. The origin of the comoving frame is the intersection of the particle at the sensor plane. The unit vectors of the comoving frame $\hat{u}_{c o}, \hat{v}_{c o}, \hat{w}_{c o}$ relative to the sensor plane can be constructed in the following way. By definition, the $w$ axis of the comoving frame is parallel to the local track direction before scattering.

$$
\hat{w}_{c o}=\frac{1}{\sqrt{1+t_{u}^{2}+t_{v}^{2}}}\left(\begin{array}{c}
t_{u} \\
t_{v} \\
1
\end{array}\right)
$$

There is a little bit of freedom to define the remaining two unit vectors of the comoving frame. Here, the following conventions are used:

$$
\begin{gathered}
\hat{v}_{c o}=\frac{\hat{w}_{c o} \times \hat{u}_{k}}{\left|\hat{w}_{c o} \times \hat{u}_{k}\right|} \\
\hat{u}_{c o}=\hat{v}_{c o} \times \hat{w}_{c o}
\end{gathered}
$$

The three unit vectors $\hat{u}_{c o}, \hat{v}_{c o}, \hat{w}_{c o}$ define the columns of a rotation matrix $R_{c o}$ between the comoving frame and the local sensor coordinates. 
A. Track fitting in the EUDET telescope with Kalman Filters

$$
R_{c o}=\left(\begin{array}{ccc}
\hat{u}_{c o} & \hat{v}_{c o} & \hat{w}_{c o}
\end{array}\right)=\left(\begin{array}{ccc}
\alpha_{1} & \alpha_{2} & \alpha_{3} \\
\beta_{1} & \beta_{2} & \beta_{3} \\
\gamma_{1} & \gamma_{2} & \gamma_{3}
\end{array}\right)
$$

The direction of the track after scattering in the comoving frame is simply given by

$$
\vec{b}_{c o}^{\star}=\left(\begin{array}{c}
\tan \theta_{1} \\
\tan \theta_{2} \\
1
\end{array}\right) \text {. }
$$

After rotating the scattered direction into the coordinates of the sensor, we obtain the following result:

$$
\begin{aligned}
t_{u}^{\star} & =\frac{\alpha_{1} \tan \theta_{1}+\alpha_{2} \tan \theta_{2}+\alpha_{3}}{\gamma_{1} \tan \theta_{1}+\gamma_{2} \tan \theta_{2}+\gamma_{3}} \\
t_{v}^{\star} & =\frac{\beta_{1} \tan \theta_{1}+\beta_{2} \tan \theta_{2}+\beta_{3}}{\gamma_{1} \tan \theta_{1}+\gamma_{2} \tan \theta_{2}+\gamma_{3}}
\end{aligned}
$$

The partial derivatives of the scatter function in the limit of vanishing scatter angles are needed as well. The result is a $4 \times 2$ matrix called noise influence matrix $G$. The non zero entries are found in Wolin and are repeated here for completeness:

$$
\begin{aligned}
& G_{1,1}=\left.\frac{\partial \lambda_{1}}{\partial \theta_{1}}\right|_{\theta_{1}, \theta_{2} \rightarrow 0}=\frac{\alpha_{1} \gamma_{3}-\alpha_{3} \gamma_{1}}{\gamma_{3}^{2}} \\
& G_{1,2}=\left.\frac{\partial \lambda_{1}}{\partial \theta_{2}}\right|_{\theta_{1}, \theta_{2} \rightarrow 0}=\frac{\alpha_{2} \gamma_{3}-\alpha_{3} \gamma_{2}}{\gamma_{3}^{2}} \\
& G_{2,1}=\left.\frac{\partial \lambda_{2}}{\partial \theta_{1}}\right|_{\theta_{1}, \theta_{2} \rightarrow 0}=\frac{\beta_{1} \gamma_{3}-\beta_{3} \gamma_{1}}{\gamma_{3}^{2}} \\
& G_{2,2}=\left.\frac{\partial \lambda_{2}}{\partial \theta_{2}}\right|_{\theta_{1}, \theta_{2} \rightarrow 0}=\frac{\beta_{1} \gamma_{3}-\beta_{3} \gamma_{1}}{\gamma_{3}^{2}}
\end{aligned}
$$

\section{Simulation of hits along the track}

The measured hit coordinates $m_{k}$ at the $k$ th sensor are given by the particle's intersection coordinates smeared with measurement noise. The relation is formally given as a so called measurement equation.

$$
m_{k}=\left(\begin{array}{c}
u_{m} \\
v_{m}
\end{array}\right)=H \lambda_{k}+\epsilon_{k}
$$

Here, the two dimensional vector $\epsilon=\left(\epsilon_{u}, \epsilon_{v}\right)$ is the measurement noise. The measurement matrix $H$ projects the local intersection coordinates out of the track parameter vector.

$$
H \lambda_{k}=\left(\begin{array}{cccc}
0 & 0 & 1 & 0 \\
0 & 0 & 0 & 1
\end{array}\right)\left(\begin{array}{cccc}
t_{u} & t_{v} & u & v
\end{array}\right)^{T}=\left(\begin{array}{c}
u \\
v
\end{array}\right)
$$

For the track model in the EUDET telescope, we will assume a purely Gaussian measurement noise. The covariance matrix of measurement noise is parametrized via the gear file from user defined intrinsic spatial resolutions. 


$$
V_{k}=<\vec{\epsilon}_{k}^{T} \overrightarrow{\epsilon_{k}}>=\left(\begin{array}{cc}
\sigma_{u}^{2} & 0 \\
0 & \sigma_{v}^{2}
\end{array}\right)
$$

\section{A.2. Kalman Filters for track fitting}

The application of the Kalman Filters to high energy particle tracking was first discussed in Frühwirth [45]. The Kalman Filter is computationally fast and flexible. If, in addition, the track model can be approximated sufficiently well by a discrete linear model in the neighborhood of the measurements belonging to an individual track, the Kalman Filter estimator has minimum variance among all linear estimators. Asymptotically, or in case of Gaussian measurement errors, this estimator is also efficient.

\section{A.2.1. Linearization for tracks in a collimated beam}

The discretized track model consists of a scattering at the current material layer and a vacuum propagation to the next material layer. The scattering at layer $k$ is given as

$$
\lambda_{k}^{\star}=g_{m s}\left(\lambda_{k}, \eta_{k}\right)
$$

while the vacuum propagation to the next layer $k+1$ is written as

$$
\lambda_{k+1}=f_{k+1 \mid k}\left(\lambda_{k}^{\star}\right)
$$

Mathematically, the track model can be seen as a composition $f_{k+1 \mid k} \bullet g_{k}$ of the scattering function and the vacuum track propagator.

$$
\lambda_{k+1}=f_{k+1 \mid k}\left(g_{m s}\left(\lambda_{k}, \eta_{k}\right)\right)
$$

Following Frühwirth [66], the idea is to linearize the track model around a reference trajectory which is a solution of the vacuum propagator and stays close to the true particle trajectory. In our case, a natural choice for a reference trajectory is the axis of the collimated particle beam. In the following, the vector $\lambda_{k, r}$ denotes the parameters of the beam axis at telescope layer $k$. To first order, the deviations between the parameters of a real particle $\lambda_{k}$ and the parameters of the beam axis can be propagated linearly.

$$
\lambda_{k+1}-\lambda_{k+1, r}=F_{k+1 \mid k}\left(\lambda_{k}-\lambda_{k, r}\right)+F_{k+1 \mid k} G_{k} w_{k}
$$

Here, $F_{k+1 \mid k}$ is the transport matrix defined as the derivative to the vacuum propagator along the beam axis.

$$
F_{k+1 \mid k}=\frac{\partial f_{k+1 \mid k}}{\partial \lambda_{k} \quad \mid \lambda_{k}=\lambda_{k, r}}
$$

Similarly, the scatter matrix $G_{k}$ is defined as the derivative of the scatter function at point $\lambda_{k, r}$.

$$
G_{k}=\frac{\partial g_{m s}}{\partial \lambda_{k}} \mid \lambda=\lambda_{k, r}
$$


The matrices $F_{k+1 \mid k}$ and $G_{k}$ were computed in the previous section. We can introduce linearized parameter vectors $x_{k}$ and linearized measurements $m_{k, r}$ in the following way:

$$
\begin{aligned}
m_{k, r} & =m_{k}-H \lambda_{k, r} \\
x_{k} & =\lambda_{k}-\lambda_{k, r}
\end{aligned}
$$

To complete the formal definition of a linearized dynamical system for Kalman filtering, the so called system equation for the parameters $x_{k}$ can be formulated as

$$
x_{k+1}=F_{k+1 \mid k} x_{k}+F_{k+1 \mid k} G_{k} w_{k}
$$

while the measurement equation is

$$
m_{k, r}=H x_{k}+\epsilon_{k} .
$$

The Kalman Filter provides estimates for the track parameter differences $x_{k}$ and their covariance matrix $C_{k}$. The predicted track parameter differences $x_{k}^{p}$ and their covariance matrix $C_{k}^{p}$ are computed using all measurement $m_{j, r}$ on upstream telescope layers $j<k$. The index $p$ indicates that the estimate is a prediction ahead in time using previous measurements on the position of the particle. Similarly, the updated parameter estimates $x_{k}^{u p}$ and $C_{k}^{u p}$ are computed using all previous measurement including the measurement at layer $k$. The recursive system of equations needed to compute predicted and updated estimates for the linear dynamical system defined in Eq. A.36 and A.34 is described in detail in the literature; see for example [78].

The Kalman Filter also requires an initial estimate $x_{0}^{p}$ and $C_{0}^{p}$ of the track parameter difference before the first measurement is taken. The method developed for this thesis is to employ the collimated beam model for filter initialization. The first layer with index $k=0$ is the $z=0$ plane and we use $x_{0}^{p}=0$ and $C_{0}^{p}=C_{b}$ (as given in Eq. A.2) to initialize the Kalman Filter. An alternative way to initialize the Kalman Filter without putting much emphasize on the beam parameters is to use $C_{0}^{p}=\alpha C_{b}$ with a large scaling factor $\alpha$. For this alternative initialization case, square root filters offer the advantage to allow an initial covariance $C_{0}^{p}$ for the limiting case $\alpha \rightarrow \infty$.

\section{A.2.2. Forward, backward and time reversed filters}

In the context of track fitting, the above described filter for the track parameters $x_{k}$ is called a forward filter. The dynamic model defined in Eq. A.36 follows the particle from layer $k=0$ to layer $k=n$ in the flight direction. The scattering of the particle at the sensor layers implies that a distinction between local parameters before and after scattering at any given layer is necessary. In particular, the forward filter estimates the parameters $\lambda_{k}$ of the particle as it enters a sensor layer before scattering takes place.

To complement the forward filter, we can define a backward filter in the following way. The linear mapping relating the particle state at layer $k+1$ to the state at layer $k$ is given as:

$$
x_{k}=F_{k+1 \mid k}^{-1} x_{k+1}-G_{k} w_{k} .
$$

Eq. A.35 can be used as the system equation for the backward filter, that allows to process mea- 
surements in the reverse order starting with the measurement at layer $k=n$. Especially, the backward filter computes predicted parameter estimates $x_{k}^{b, p}$ using all measurements at layer $j>k$ and updated estimates $x_{k}^{b, u p}$ using all measurements $j \geqq k$.

There is another possibility to run the dynamic system given in Eq. A.33 in the opposite direction, namely time reversal. The system equation for the time reversed dynamic is given as

$$
x_{k}^{\star}=F_{k \mid k+1} x_{k+1}^{\star}+F_{k \mid k+1} G_{k+1} w_{k+1} .
$$

Obviously, the time reversed system must be formulated for the state of the particle $x_{k}^{\star}$ after scattering at the material in layer $k$. Using the notion developed in the previous section, we can write this in the following way:

$$
x_{k}^{\star}=\lambda_{k}^{\star}-\lambda_{k, r}
$$

The time reversed system can be used to process measurement in the reverse order starting with the measurement at layer $k=n$ and processing against the flight direction of the particle. Unlike the backward filter, the time reversed filter estimates the local particle state after scattering at layer $k$. In this thesis, the main use of the time reversed filter is to estimate the scattering kinks $\eta_{k}$ from differences $\Delta_{k}$ between the prediction of the forward filter $x_{k}^{p}$ and the prediction of the time reversed filter $x_{k}^{\star, p}$.

$$
\Delta_{k}=x_{k}^{\star, p}-x_{k}^{p}
$$

As the forward filter uses all measurements at sensor before layer $k$ and the time reversed filter uses all measurements behind, it is obvious that $x_{k}^{\star, p}$ and $x_{k}^{p}$ are uncorrelated. As a consequence, the covariance for the difference $\Delta_{k}$ is simply given by

$$
C_{\Delta_{k}}=C_{k}^{\star, p}+C_{k}^{p}
$$

\section{A.2.3. Track fitting with forward and backward filters}

By running a forward and a backward filter at the same time, we can produce precise estimators for track parameters at layer $k$ using hits before and after layer $k$ at the same time. To simplify the notation, we will drop the uppercase index $p$ and denote the predicted estimators from the forward and backward filters as $x_{k}^{f}, C_{k}^{f}$ and $x_{k}^{b}, C_{k}^{b}$. By construction, the these estimators are uncorrelated for any layer $k$ because they use non-overlapping sets of measurements. Therefore, we can compute a smoothed estimator for the local parameters at layer $k$ as a weighted means.

$$
\begin{gathered}
C_{k}=\left(\left(C_{k}^{f}\right)^{-1}+\left(C_{k}^{b}\right)^{-1}\right)^{-1} \\
x_{k}=C_{k}\left(\left(C_{k}^{f}\right)^{-1} x_{k}^{f}+\left(C_{k}^{b}\right)^{-1} x_{k}^{b}\right)
\end{gathered}
$$

The smoothed parameter vector $x_{k}$ and its covariance $C_{k}$ combine the information from all measurements excluding the measurement at layer $k$ itself. In other words, $x_{k}$ and $m_{k}$ are uncorrelated. The smoothed track parameter vector $\lambda_{k}$ at the $k$ th sensor is obtained as the sum $x_{k}+\lambda_{k, r}$ and has the 


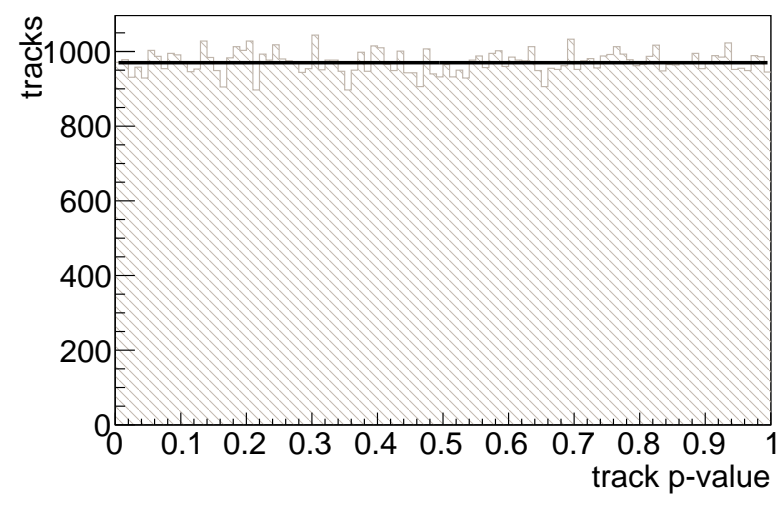

Figure A.1.: Distribution of track $p$-values for simulated tracks from 100 randomly tilted telescope setups in a $1 \mathrm{GeV}$ electron beam.

covariance matrix $C_{k}$ defined in Eq. A.40. For track fitting in the EUDET telescope, the fitting method is implemented in a way to compute smoothed local track parameters for all sensors in the telescope.

\section{A.3. Validation of track fitting with pseudo experiments}

A dedicated Marlin processor DEPFETTrackingTest has been developed to validate the track fitting. The processor reads the telescope geometry from the same gear file as used for the reconstruction of real data. The Marlin steering file allows to specify the properties of the particle beam like the beam momentum, beam spot size and others. The processor outputs a root file with detailed control plots containing track residuals, track parameter pulls and the $p$-value distribution.

The track fitting algorithm was tested by simulating the EUDET telescope in a beam of $1 \mathrm{GeV}$ electrons similar to test beam setups at DESY. The simulated telescope contains seven sensors with properties close to the specifications of Mimosa26 sensors. Especially, the simulated intrinsic spatial resolution was $3.5 \mu \mathrm{m}$ and the radiation length $T / T_{0}$ was $0.07 \%$. The distance between sensors was chosen to be $20 \mathrm{~mm}$ and the innermost sensor is considered as the device under test. In the simulation, all sensors were randomly tilted in the range $-20^{\circ} \leq \alpha \leq+20^{\circ},-20^{\circ} \leq \beta \leq+20^{\circ}$ and $-20^{\circ} \leq \gamma \leq+20^{\circ}$ around the global $x, y$ and $z$ axes. The initial track parameters were generated at random directions and positions covering the full acceptance of the telescope and leading to hits at all six sensor planes. The simulation was carried out for 100 random telescope geometries and 1,000 tracks were simulated for each telescope.

The histogram of track $p$-values is shown in Fig. A.1. The $p$-value distribution is flat and follows perfectly the theoretical expectation for the case that all noise sources, scattering kinks and measurement errors, are modeled by uncorrelated Gaussian. The result shows that the linearization scheme works well and the remaining non linearity can indeed by neglected. The pull values from the track fits for all the four track parameters at the center of the telescope are shown in Fig. A.2. The curves fitted to the histograms are Gaussian from which it can be seen that the pull values follow very well the Gaussian distribution with zero mean and unit variance. This proves the validity of the fit procedure. In the center of the telescope, the track fitter gives an error for the position parameters $u$ and $v$ of about $3.5 \mu \mathrm{m}$ and for the slope parameters $t_{u}$ and $t_{v}$ the error is $1.6 \times 10^{-4} \mathrm{rad}$. 
A. Track fitting in the EUDET telescope with Kalman Filters
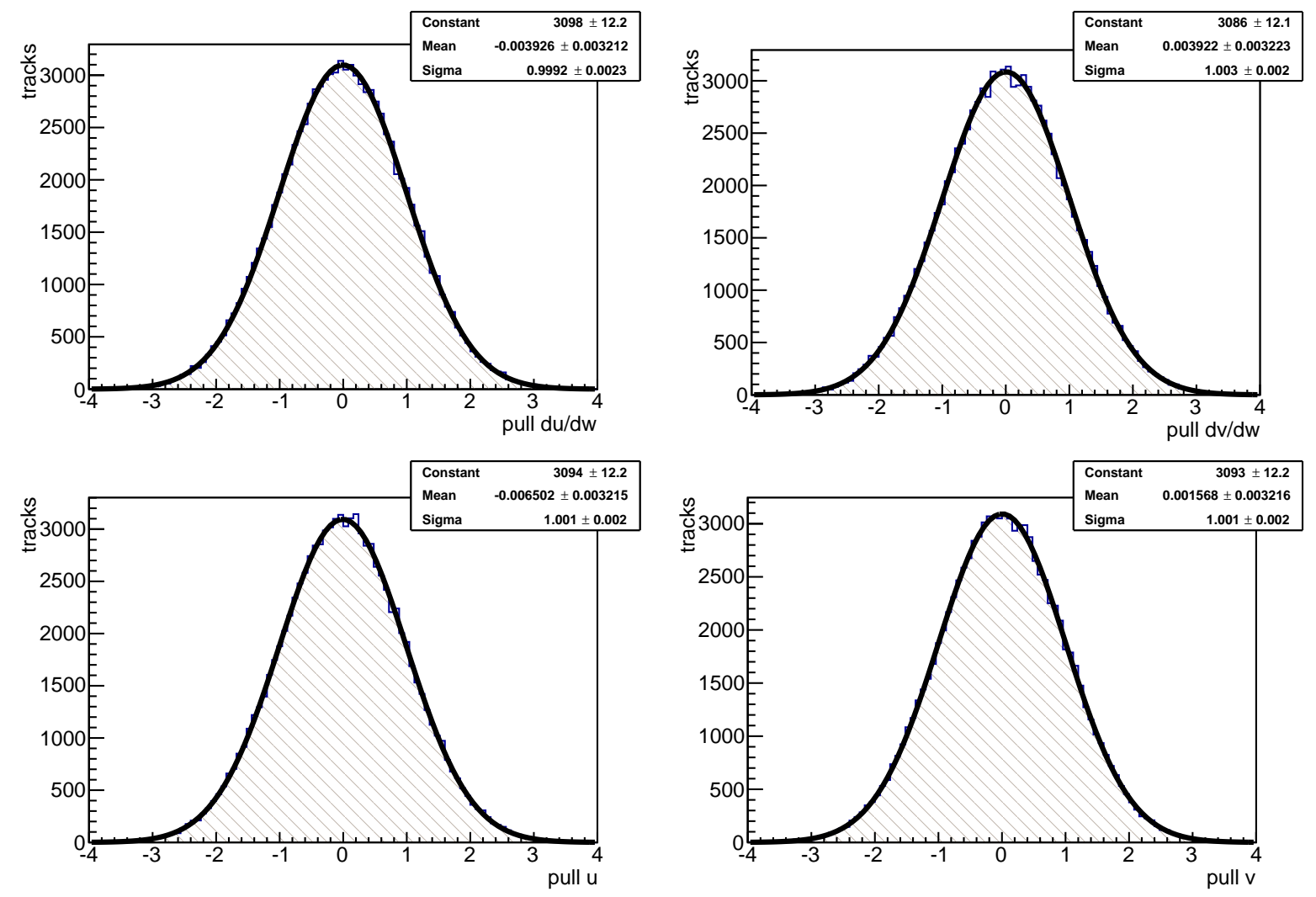

Figure A.2.: Distribution of pull values for the track parameters at the central sensor plane in the simulated telescope. Pull values are defined as standardized differences between estimated and simulated parameter values. The pulls are fitted to a Gaussian. The fitted means are centered around zero and the fitted sigma is close to one. 


\section{B. Kalman Alignment Algorithm with annealing}

The Kalman Filter technique offers a way to minimize the global $\chi^{2}$ function defined in Eq. 4.22 by a sequential processing of tracks. The idea to utilize the Kalman Filter update rule to estimate both track parameters and telescope alignment parameters at the same time was first proposed by Frühwirth [18] in 2003. In this work, the Kalman Filter Alignment Algorithm (KAA) was applied to the problem of aligning a simulated strip telescope from high energy track data where multiple scattering is negligible. Here, we outline the mathematical details to implement the Kalman Alignment Algorithm for the alignment of the EUDET telescope from low energy track data available for test beams at DESY. In the last part of this appendix, we show the results of pseudo experiments to validate the alignment algorithm. The application of the algorithm on real data has already been shown (section 4.5.4.2).

\section{B.1. The sequential update rule}

The Kalman Alignment Algorithm views every measured particle track as a further piece of information to obtain a better telescope geometry. In order to clearly formulate the algorithm, we have to introduce a notation that allows us to keep track of the sequence of different telescope geometries obtained during the alignment.

The track sample used for alignment consists of $n$ tracks recorded by the beam telescope. The hit of track $j$ on sensor $i$ is a $2 D$ vector $m_{i j}$ and has a $2 \times 2$ covariance matrix $V_{i j}$. For telescope alignment, we will use tracks crossing the entire telescope with hits on all sensors.

The initial telescope geometry $\left\{r_{i}, R_{i}\right\}_{0}$ specifies the positions of all sensors before the first track is processed. The initial vector of alignment parameters is $a_{0}=0$. The initial covariance matrix $E_{0}$ of alignment parameters quantifies our belief about the errors in the initial telescope geometry. After processing the first $j$ tracks in the track sample, the alignment parameters are denoted as $a_{j}$ having an covariance matrix $E_{j}$. Applying the alignment parameters $a_{j}$ to the initial geometry, we obtain a corrected telescope geometry $\left\{r_{i}, R_{i}\right\}_{j}$.

In the following, we denote the smoothed track parameters of track $j$ at sensor $i$ with $\lambda_{i j}$. The track parameter covariance matrix is $C_{i j}$. As discussed before (see section A.2.3, the smoothed track parameters $\lambda_{i j}$ are uncorrelated with the hit $m_{i j}$. The track fit which yields the smoothed track parameters $\lambda_{i j}$ is performed in the telescope geometry $\left\{r_{i}, R_{i}\right\}_{j-1}$ obtained after processing the first $j-1$ tracks.

The advantage to update the telescope geometry after each processed track is to mitigate the effect of sensor misalignment on track fitting. In case the alignment converges, the updated geometry is always closer to the real geometry and reduces the bias on local track parameters $\lambda_{i j}$. In close vicinity to the true telescope geometry, the local track parameters are unbiased and the parameter errors are dominated by random measurement errors and scattering kinks. 


\section{B. Kalman Alignment Algorithm with annealing}

For the local alignment update, we use the hit $m_{i j}$ at sensor $i$ from track $j$ to improve our information on the local track parameters $\lambda_{i j}$ and the local alignment state $a_{i j}$ at the same time. We can formulate a measurement equation for the joint alignment and track fitting problem as

$$
m_{i j}=h_{i j}\left(\lambda_{i j}, a_{i j}\right)+\epsilon_{i j}
$$

where $\epsilon_{i j}$ is the measurement noise of sensor $i$. In order to cast the measurement equation in the standard form for Kalman filters, we define a concatenated parameter vector $x_{i j}=\left(\lambda_{i j}, a_{i j}\right)$ with a covariance matrix

$$
B_{i j}=\left(\begin{array}{cc}
C_{i j} & 0 \\
0 & E_{i j}
\end{array}\right)
$$

The block diagonal form of $B_{i j}$ implies that track and alignment parameters are considered as uncorrelated. An important consequence of the block diagonal form is that the update for alignment and track parameters decouples. Since they are of no further interest, the computation of the updated track parameters can be skipped. The Kalman Filter measurement update for the alignment parameters and their covariance matrix can be written as

$$
\begin{aligned}
a_{i j+1} & =a_{i j}+E_{i j} D_{i j}^{T} W_{i j}\left[m_{i j}-h_{i j}\left(\lambda_{i j}, a_{i j}\right)\right] \\
E_{i j+1} & =E_{i j}-E_{i j} D_{i j}^{T} W_{i j} D_{i j} E_{i j}^{T} .
\end{aligned}
$$

The following auxiliary matrix needs to be computed.

$$
W_{i j}=\left(V_{i j}+H_{i j} C_{i j} H_{i j}^{T}+D_{i j} E_{i j} D_{i j}^{T}\right)^{-1}
$$

The matrices $H_{i j}$ and $D_{i j}$ are the derivatives of the measurement equation $h_{i j}$ with respect to local track and alignment parameters.

$$
\begin{aligned}
H_{i j} & =\frac{\partial h_{i j}}{\partial \lambda_{i j}} \\
D_{i j} & =\frac{\partial h_{i j}}{\partial a_{i j}}
\end{aligned}
$$

In the case of using local track parameters $\lambda_{i j}=\left(t_{u}, t_{v}, u, v\right)$, the derivatives $H_{i j}$ are constant and have already been computed, see Eq. A.23. The alignment derivatives $D_{i j}$ can be computed in two steps. The derivatives $D_{i j}^{l o c}$ of the measurement equation with respect to the local alignment vector $a_{i j}^{l o c}=(\Delta u, \Delta v, \Delta w, \Delta \alpha, \Delta \beta, \Delta \gamma)$ have been computed in Karimäki [69].

$$
D_{i j}^{l o c}=\left(\begin{array}{cccccc}
-1 & 0 & t_{u} & -v t_{u} & u t_{u} & -v \\
0 & -1 & t_{v} & -v t_{v} & u t_{v} & u
\end{array}\right)
$$

The derivatives with respect to the alignment parameters $a_{i j}=(\Delta x, \Delta y, \Delta z, \Delta \alpha, \Delta \beta, \Delta \gamma)$ can be obtained via the chain rule. 


$$
D_{i j}=\frac{\partial h_{i j}}{\partial a_{i j}^{l o c}} \frac{\partial a_{i j}^{l o c}}{\partial a_{i j}}=D_{i j}^{l o c} A_{i j}
$$

The auxiliary $6 \times 6$ matrix $A_{i j}$ can be computed as:

$$
A_{i j}=\frac{\partial a_{i j}^{l o c}}{\partial a_{i j}}=\left(\begin{array}{cc}
R_{i} & 0_{3 \times 3} \\
0_{3 \times 3} & 1_{3 \times 3}
\end{array}\right) .
$$

The matrix $R_{i}$ is the rotation matrix of sensor $i$. The advantage of using $\Delta x, \Delta y$ and $\Delta z$ as alignment parameters instead of $\Delta u, \Delta v$ and $\Delta w$ becomes visible for tilted sensors where $\alpha_{i} \neq 0$ or $\beta_{i} \neq 0$. The sensor tilts give rise to strong correlations between the two variable pairs $\Delta u, \Delta w$ and $\Delta v, \Delta w$. These correlations can be avoided by using the variables $\Delta x, \Delta y$ and $\Delta z$ instead.

\section{B.2. The initial alignment covariance and annealing}

The Kalman Filter alignment method needs an initial alignment covariance matrix $E_{0}$ to start the update procedure. In practice, we restrict ourselves to a diagonal covariance matrix and have the freedom to set an initial variance for each alignment parameter. In practice, this freedom can be used in three different ways:

- The expected errors of the initial sensor positions are summarized in Tab 4.4. We can use the square of these number as an initial covariance to start the alignment. This is particularly useful for the rotations $\Delta \alpha$ and $\Delta \beta$ in order to avoid large excursions of these parameters at the beginning of alignment. In case of further iterations of the alignment, the value of the initial variance should be decreased slowly.

- The Kalman Alignment Algorithm allows to set the initial alignment of certain alignment parameters to zero. This choice implies that these parameters cannot be changed by track data during alignment. In particular, the eight weak mode parameters of the telescope can be constrained by assigning zero variance to the $\Delta x, \Delta y, \Delta z$ and $\Delta \gamma$ degrees of freedom of the first and the last sensor in the beam. In this case, the weak mode deformations present in the nominal geometry remain unchanged.

- It is also possible to assign zero variance to all alignment parameters of one or more sensors in the telescope. For example, this allows a standalone alignment of a device under test with respect to a previously aligned reference telescope.

The local alignment method is attractive as it decomposes the alignment of the whole telescope into the alignment of several independent sensors. However, the local alignment risks to converge into a shallow local minimum of the global $\chi^{2}$ function. For a beam telescope, the occasional convergence to a local minimum was observed in Frühwirth [18]. The suggested solution in Frühwirth is a geometric annealing scheme. Geometric annealing means that the hits are down-weighted by a large factor in the beginning of alignment. This factor is then gradually decreased, until it reaches unity after a prescribed number of tracks. More precisely, we can formulate annealing as a rescaling of the hit covariance matrix for hits in track $j$. 


$$
V_{i j} \rightarrow \alpha(j) V_{i j}
$$

Here, $\alpha(j)$ denotes the annealing function. It is important that the rescaled covariance matrices must be used both in the track refit to obtain $\lambda_{i j}$ and in the local alignment update to obtain $a_{i j+1}$ and $E_{i j+1}$. Intuitively, annealing tries to absorb the misplacement of sensors into a blown up measurement error. Consequently, the initial annealing factor depends on the scale of misplacement of sensors and must be reduced once the position of sensors gradually shrinks during the alignment process. Obviously, well aligned sensors should not be annealed and annealing should stop well before the end of the track sample is reached. Different types of annealing are possible. However, a good choice for the annealing function is:

$$
\alpha(j)= \begin{cases}b^{\frac{m-j}{m-1}} & , j<m \\ 1 & j \geqq m\end{cases}
$$

The geometric annealing function depends on two parameters. Annealing is turned off after $m$ tracks and the initial annealing factor is $b$. The optimal values of $m$ and $b$ should be tuned for each alignment problem by simulation studies taking into account the actual telescope geometry and the available track sample. However, experience shows that annealing functions using $m \sim 100-1000$ tracks and an initial annealing factor $b \sim 1000-10,000$ give good results.

\section{B.3. Validation of telescope alignment}

A Monte Carlo simulation was written to test the alignment algorithm in a standalone Marlin processor called DEPFETAlignerTest. The approach to validate the simulation follows closely the prescriptions given in Karimäki [69]. The simulation takes an initial design telescope geometry and randomly moves and rotates all sensor planes according to mechanical installation errors. Particle tracks were simulated and followed through the beam telescope. Our imperfect knowledge of the true position of the sensor planes is simulated by reconstructing the tracks in the design telescope geometry. This means that the track fit uses the wrong transformation from local to global coordinates and leads to biased track parameters. The full algorithm runs as follows:

1. Creation of a ideal detector with no misalignment

2. Creation of a misaligned (realistic) detector

3. Generation of particles and hits in the misaligned detector

4. Alignment procedure using hits (generated in misaligned detector) but starting alignment in ideal detector

5. Validation of aligned detector using an independent sample of tracks

The simulated telescope has six Mimosa26 sensors. The spacing between all sensors is $40 \mathrm{~mm}$. The misalignment shifts $\Delta x, \Delta y, \Delta z$ are chosen at random, each in the range $\pm 2 \mathrm{~mm}$. The misalignment 


\section{B. Kalman Alignment Algorithm with annealing}

tilts $\Delta \alpha, \Delta \beta, \Delta \gamma$ were chosen at random in the range $\pm 100 \mathrm{mrad}$. The telescope is positioned in a way such that the beam axis crosses all sensors planes and particle tracks cover the entire active sensor area. For the track sample used for alignment, we assume a beam with $2 \mathrm{GeV}$ electrons. The size of the beam spot is $20 \mathrm{~mm}$ and the beam divergence is $3.5 \mathrm{mrad}$.

In reality, all sensors in the telescope have positioning errors and should be aligned. However, in order to avoid complications from global telescope distortions, we keep the position of the first and the last sensor at their design values. In the alignment, the position of these two sensors is fixed and defines a reference coordinate system for the alignment of the inner sensors. In order to study the statistical properties of the alignment method, the simulations is repeated for 250 randomly misaligned telescope geometries. For each case, we simulate a sample of 10, 000 tracks for alignment and another 2000 tracks are simulated for the validation of the alignment results. The alignment proceeds in three iterations or passes over the track sample:

- First Pass: Alignment of the robust $\Delta x, \Delta y, \Delta \gamma, \Delta z$ degrees of freedom of the inner telescope sensors. The initial variances for these parameters are set to the mechanical installation uncertainty (RMS). All other alignment parameters are fixed by assigning a zero initial covariance. The geometric annealing schedule is used.

- Second Pass: Alignment of all six degrees of freedom per sensor. Initial variances for alignment parameters are set according to the installation uncertainty (RMS). The geometric annealing schedule is used.

- Third Pass: Similar to the second pass but with reduced values for the initial variances of alignment parameters. The initial alignment covariance matrix scaled down by a factor of $1 / 4$.

The convergence of the alignment method is very fast due to the combination of annealing and the immediate application of alignment corrections after each track. The track fit $\chi^{2}$ distribution after alignment in Fig. B.1 agrees well the the theoretical expectation of a proper $\chi^{2}$ distribution with 8 degrees of freedom. The precision of the fitted parameters in comparison with the true values is shown in Fig. B.2. As can be seen, the correlations between aligned and true parameter are very strong. The typical deviation of the fitted parameters from the true value is less than $1 \mu \mathrm{m}$ for the offsets and a fraction of a milliradian for the tilts. The precision appears to be better than actually needed in this case, indicating that a smaller track sample would give a satisfactory result.

Finally, we have checked whether the computed errors on the alignment parameters correspond to the actual spread around the true values. The resulting histograms of the standardized residuals (pulls) are shown in Fig. B.3. The standard deviations of pulls for all alignment parameters are reasonably close to unity. As a conclusion, all six degrees of freedom could be aligned in the simulation experiment. However, the simulation assumed a beam with an angular divergence of $3.5 \mathrm{mrad}$ which is at least two times larger than the beams available at DESY or CERN. A track sample from a less divergent beam gives worse results for the correction of sensor $z$ positions and tilt angles $\alpha$ and $\beta$. The multiple scattering of $2 \mathrm{GeV}$ electrons poses no problem for telescope alignment. In fact, multiple scattering makes the particle beam more divergent and gives better sensitivity for the correction of sensor tilts and $z$ positions. 
B. Kalman Alignment Algorithm with annealing

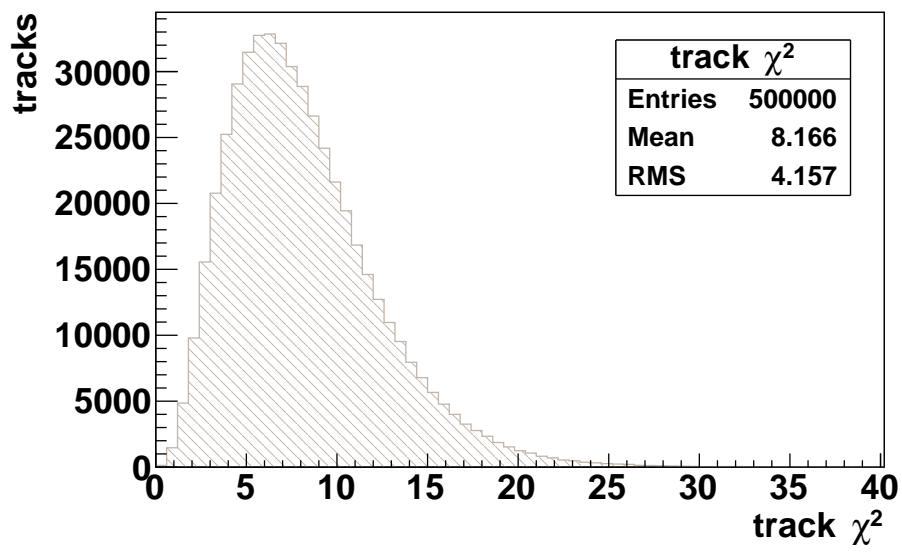

Figure B.1.: Distribution of track $\chi^{2}$ values for simulated tracks from 100 randomly tilted telescope setups in a $3 \mathrm{GeV}$ electron beam. The sample mean and RMS values agree well with the theoretical expectation of a proper $\chi^{2}$ distribution with 8 degrees of freedom. 

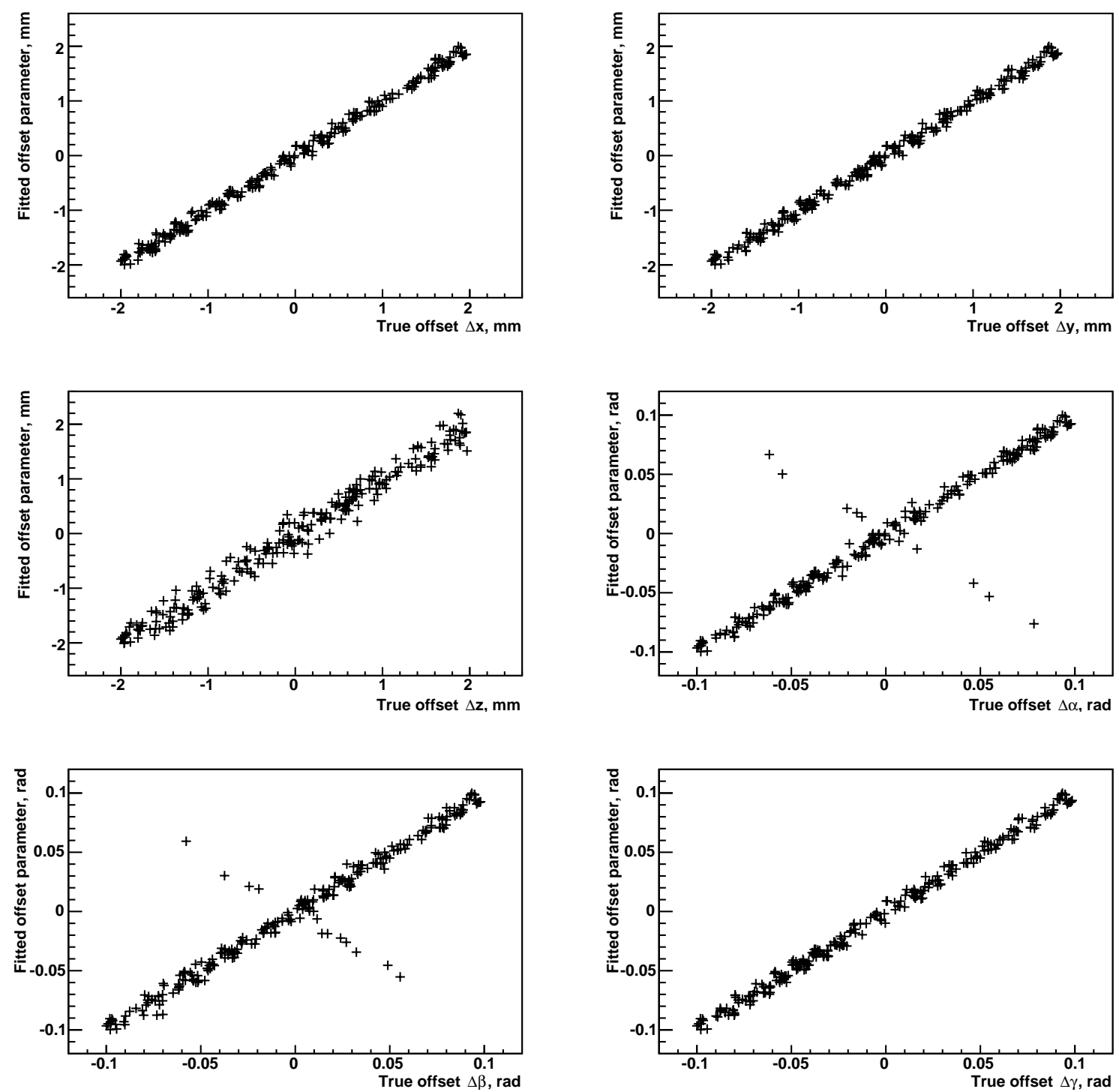

Figure B.2.: Scatter plot of the fitted and true alignment parameters at a central sensor plane in the misaligned telescope. The alignment experiment is repeated 250 times with randomly displaced sensors. A strong correlation between the fitted and true parameters is visible. In rare cases, fitted tilt angles converge to the wrong sign. 

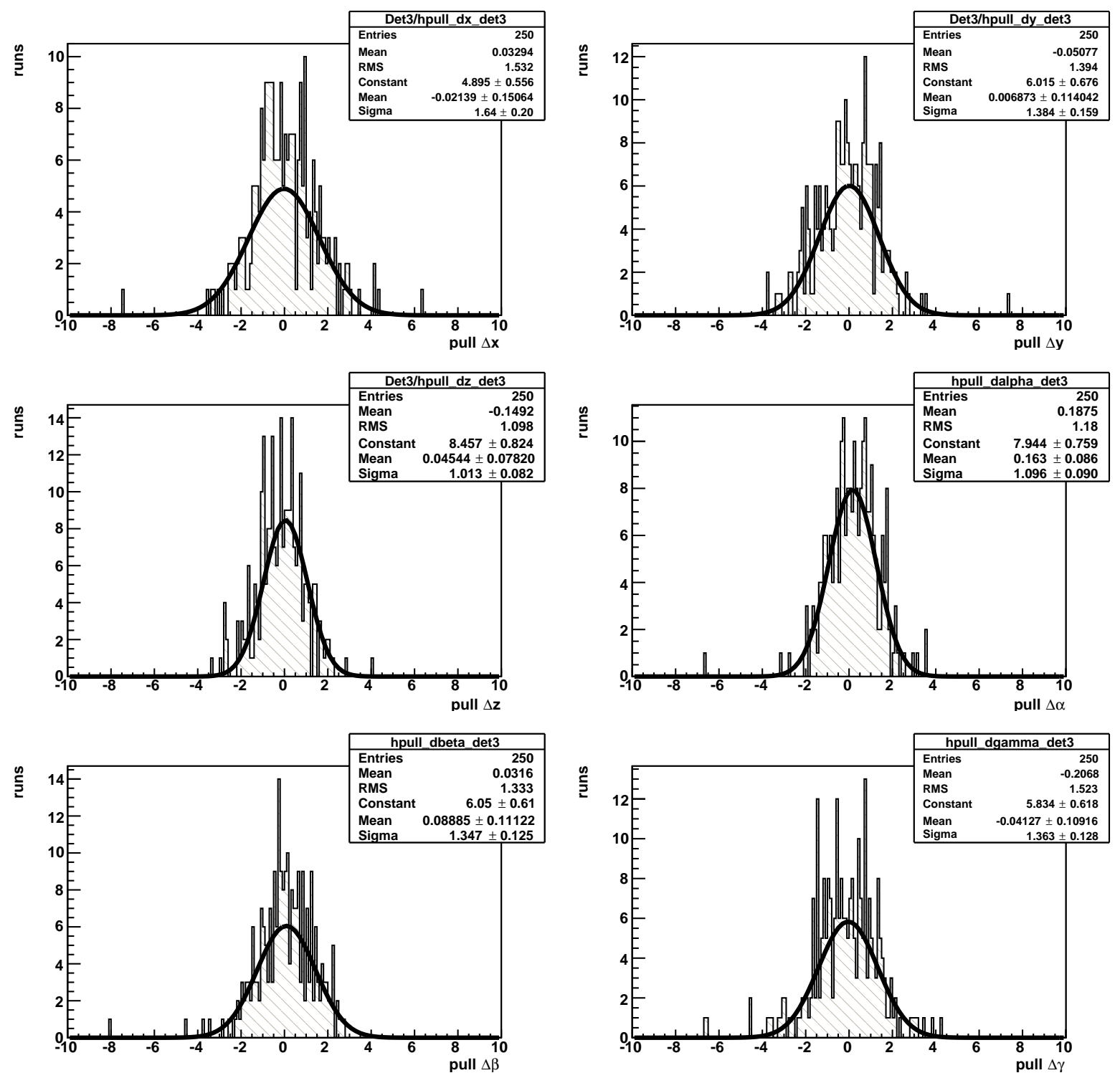

Figure B.3.: Distribution of pull values for the alignment parameters at a central sensor plane in the misaligned telescope. Pull values are defined as standardized differences between estimated and simulated (true) parameter values sampled in 250 runs. The pulls are fitted to a Gaussian. The fitted means are centered around zero and the fitted sigma is close to one. 


\section{GEAR interface for test beams}

A GEAR file is used to describe the geometry of the beam telescope needed for data reconstruction and simulation. The GEAR file is organized as an array of so-called layers. A layer represents a detector module installed in the telescope as a bounded plane which has an outer ladder and an inner sensitive part. The data fields in the ladder part are used to describe the materials around the sensor element including for example the printed circuit board. The model for the ladder allows to specify the length width, thickness and radiation length of materials around the sensor. The data fields in the sensitive part are used to describe the geometrical layout of the sensor element and its position in the telescope. There are additional data fields to specify an intrinsic spatial resolution of the sensor element along the local $u$ and $v$ direction 1

The general layout of the GEAR files used throughout this thesis is shown in Listing C.1. The example file shows the specification of GEAR layers for a single Mimosa26 or DEPFET module in the beam telescope. The design of the GEAR interface allows to add an arbitrary number of additional telescope layers.

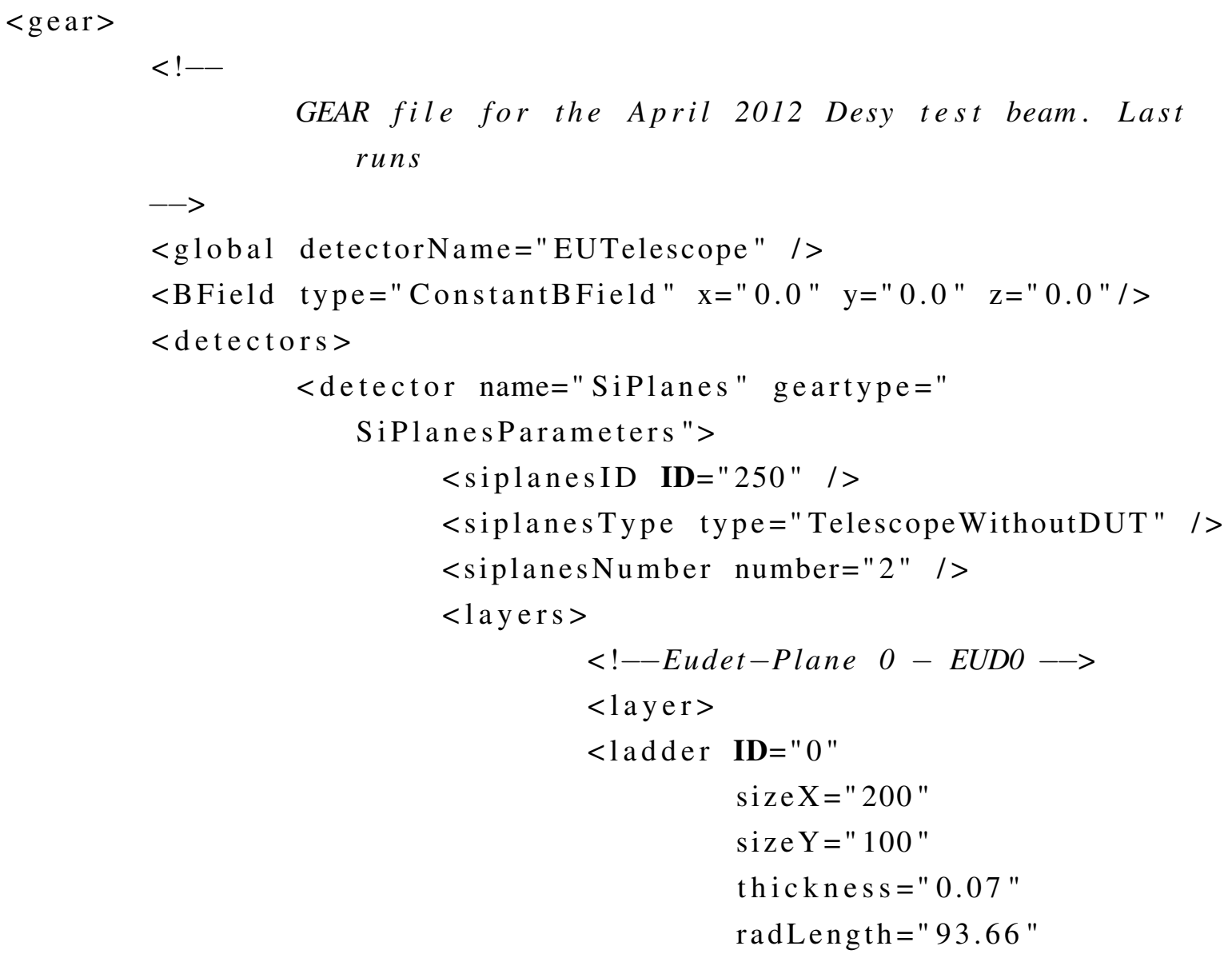

${ }^{1}$ The $x$ and $y$ axes of a layer are internally interpreted as local $u$ and $v$ axes. The names in the Gear file are kept for consistency reasons. 
C. GEAR interface for test beams

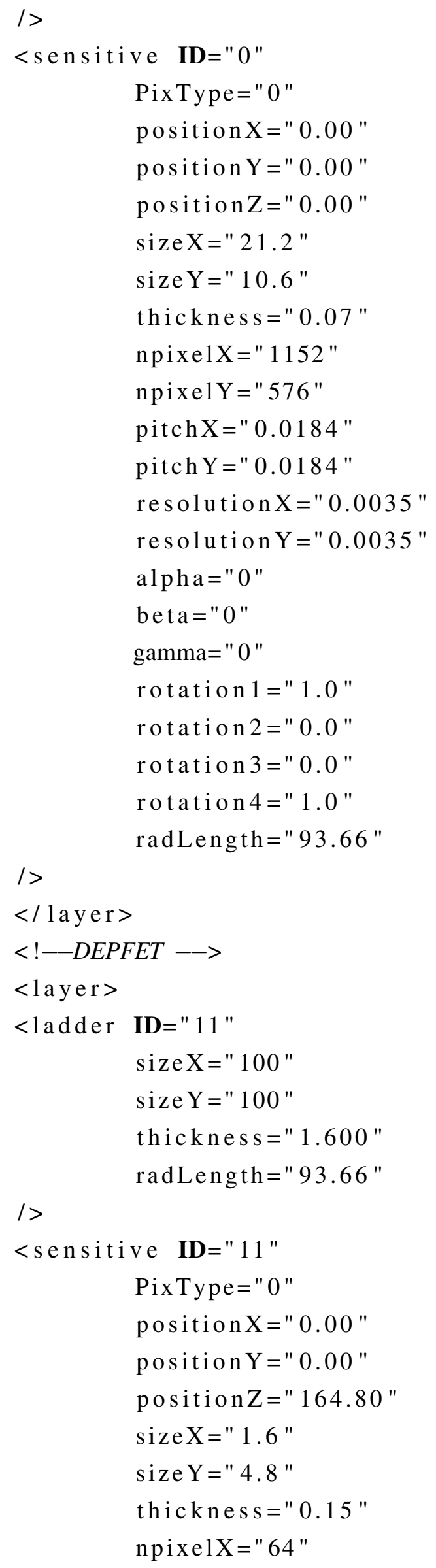


C. GEAR interface for test beams

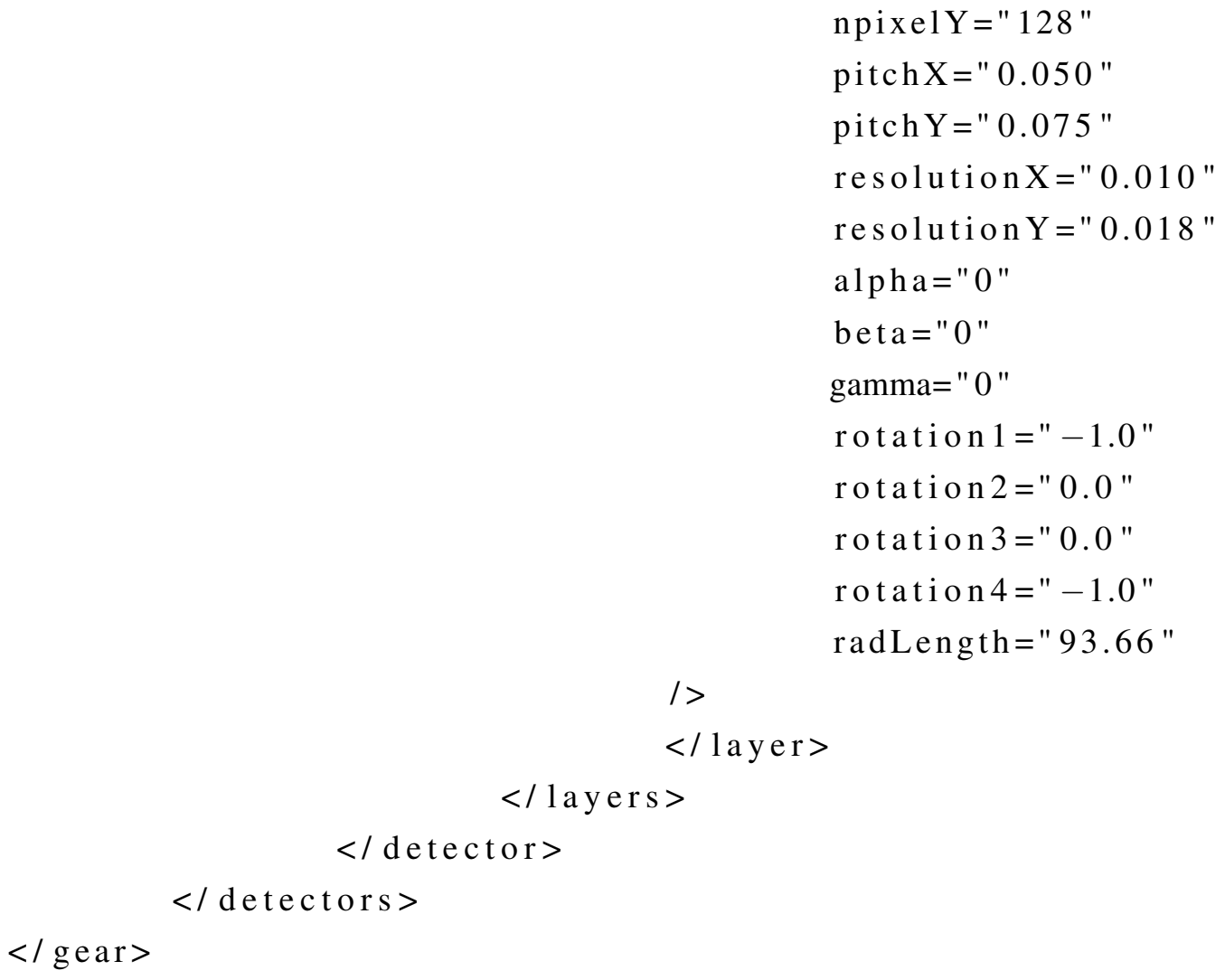

Listing C.1: Layout for gear file of a simplified beam telescope with a Mimosa26 plane and a DEPFET module. 


\section{Acknowledgments}

First of all, I would like to thank Prof. Dr. Ariane Frey for giving me the opportunity to write my thesis in particle physics in her research group. I want to thank her for giving me the freedom to make my own research decisions while at the same time taking care that these decisions do not lead me too far astray. It has been a very valuable experience to be working as colleague among the fellow scientists in her research group. I also want to thank Prof. Dr. Arnulf Quadt and PD Dr. Jörn Große-Knetter for their co-supervision of my $\mathrm{PhD}$ project during the last years.

Of course I want to thank the members of our institute, for giving me such a nice time during working hours and beyond. I want to thank Jens Weingarten for his careful and patient proof-reading of my manuscript. Jörn Große-Knetter taught me that a good espresso served at a friendly place helps to forget about a bad lunch. I want to thank Christian Geisler, Fabian Wilk and Ulf Stolzenberg for their great enthusiasm during stressful test beam campaigns at DESY and CERN and many fruitful discussions in our office and elsewhere.

Thanks to Christa Wohlfahrt, Bernadette Tyson, Heidi Afshar, Heike Ahrens and Gabriela Herbold for constant and constructive help with administrative issues. Special thanks to Lucie Hamdi for her patience with creatively filled or even delayed travel forms.

Thanks a lot to all the people from the DEPFET community for the lively and inspiring atmosphere during the workshops and the comradeship at test beams. I am grateful to Zbynek Drasal who introduced me to his work on detector simulations and provided me with a starting point for my own research. I want to express my special gratitude to Rainer Richter, from whom I learned much about the operation and design of DEPFET detectors. Special thanks go to Marcel Vos, Carlos Marinas, Florian Lütticke, Christian Koffmane and the whole DEPFET test beam crew for bringing our detectors to life before our time slots ended.

Many thanks to my friends Robin, Sascha, Thomas, Freya, Felix and Satu for cheering me up and making me smile whenever I was low spirited. I am very grateful to my parents for their constant support - not only, but also - during my PhD time. 


\section{Bibliography}

[1] F. Englert and R. Brout, "Broken Symmetry and the Mass of Gauge Vector Mesons," Phys. Rev. Lett. 13 (1964) 321-323.

[2] P. W. Higgs, "Broken Symmetries and the Masses of Gauge Bosons," Phys. Rev. Lett. 13 (1964) 508-509.

[3] ATLAS Collaboration, G. Aad, et al., "Observation of a new particle in the search for the Standard Model Higgs boson with the ATLAS detector at the LHC," Phys.Lett. B716 (2012) 1-29.

[4] CMS Collaboration, S. Chatrchyan, et al., "Observation of a new boson at a mass of $125 \mathrm{GeV}$ with the CMS experiment at the LHC," Phys.Lett. B716 (2012) 30-61.

[5] M. Kobayashi and T. Maskawa, "CP-Violation in the Renormalizable Theory of Weak Interaction," Prog. Theor. Phys. 49 no. 2, (1973) 652-657.

[6] A. Sakharov, "Violation of CP Invariance, c Asymmetry, and Baryon Asymmetry of the Universe,” Pisma Zh.Eksp.Teor.Fiz. 5 (1967) 32-35.

[7] A. Riotto, "Theories of baryogenesis," arXiv: hep-ph/9807454 [hep-ph].

[8] B. Aubert et al., "The BaBar detector," Nucl.Instrum.Meth. A479 (2002) 1-116.

[9] A. Abashian et al., "The Belle Detector," Nucl.Instrum.Meth. A479 (2002) 117-232.

[10] Letter of Intent for KEK Super B Factory: SuperKEKB Task Force. Letter of Intent. KEK, Tsukuba, 2004.

[11] T. Abe et al., "Belle II Technical Design Report," arXiv:1011.0352 [physics.ins-det].

[12] J. Kemmer and G. Lutz, "New detector concepts," Nucl.Instrum.Meth. A253 no. 3, (1987) 365 377 .

[13] H. G. Moser et al., "Thinned Silicon Detectors,” PoS Vertex 2007 (2007) .

[14] Z. Drasal, K. Prothmann, and B. Schwenker, "Silicon simulation code for Belle II and ILC," PoS VERTEX 2011 (2011) .

[15] J. Knopf et al., “A 256 channel 8-Bit current digitizer ASIC for the Belle-II PXD,” JINST 6 no. $01,(2011)$.

[16] I. Peric, P. Fischer, and T. H. H. Nguyen, "DCDB and SWITCHERB, the readout ASICS for Belle II DEPFET pixel detector,” IEEE Nucl.Sci.Symp.Conf.Rec. 2011 (2011) 1536-1539. 
[17] I. Rubinskiy, “An EUDET/AIDA Pixel Beam Telescope for Detector Development ," Physics Procedia 37 no. 0, (2012) 923 - 931. Proceedings of the 2nd International Conference on Technology and Instrumentation in Particle Physics (TIPP 2011).

[18] R. Frühwirth et al., "Estimation of detector alignment parameters using the Kalman filter with annealing," J. Phys. G29 no. 3, (2003) 561.

[19] R. A. Millikan, "The Isolation of an Ion, a Precision Measurement of its Charge, and the Correction of Stokes's Law," Phys. Rev. (Series I) 32 (1911) 349-397.

[20] A. Einstein, "Über einen die Erzeugung und Verwandlung des Lichtes betreffenden heuristischen Gesichtspunkt," Annalen der Physik 322 (1905) 132-148.

[21] H. R. Hertz, "Über einen Einfluss des ultravioletten Lichtes auf die elektrische Entladung," Annalen der Physik 31 (1887) 983.

[22] R. P. Feynman, "Space-Time Approach to Non-Relativistic Quantum Mechanics," Rev. Mod. Phys. 20 (1948) 367-387.

[23] S.-I. Tomonaga and J. R. Oppenheimer, "On Infinite Field Reactions in Quantum Field Theory," Phys. Rev. 74 (1948) 224-225.

[24] J. Schwinger, "Quantum Electrodynamics. III. The Electromagnetic Properties of the Electron Radiative Corrections to Scattering," Phys. Rev. 76 (1949) 790-817.

[25] R. Feynman and A. Zee, QED: The Strange Theory of Light and Matter. Princeton Science Library. Princeton University Press, 2014.

[26] D. Griffiths, Introduction to Elementary Particles. Physics textbook. Wiley, 2008.

[27] A. Seiden, Particle Physics: A Comprehensive Introduction. Pearson Education, 2004.

[28] Particle Data Group, K. Nakamura, et al., "Review of Particle Physics," Phys. Rev. D86 (2012)

[29] H. D. Politzer, "Reliable Perturbative Results for Strong Interactions?," Phys.Rev.Lett. 30 (1973) 1346-1349

[30] H. D. Politzer, "Asymptotic Freedom: An Approach to Strong Interactions," Phys.Rept. 14 (1974) 129-180.

[31] D. Gross and F. Wilczek, "Asymptotically Free Gauge Theories. 1," Phys.Rev. D8 (1973) $3633-3652$

[32] S. Glashow, "Partial Symmetries of Weak Interactions," Nucl.Phys. 22 (1961) 579-588.

[33] A. Salam and J. C. Ward, "Weak and electromagnetic interactions," Nuovo Cim. 11 (1959) 568-577.

[34] S. Weinberg, "A Model of Leptons," Phys. Rev. Lett. 19 (1967) 1264-1266. 
[35] J. Christenson et al., "Evidence for the 2 pi Decay of the k(2)0 Meson," Phys.Rev.Lett. 13 (1964) 138-140.

[36] CKMfitter Group, J. Charles, et al., "CP violation and the CKM matrix: assessing the impact of the asymmetric $B$ factories," Eur.Phys.J. C41 no. 1, (2005) 1-131.

[37] T. Aushev et al., "Physics at Super B Factory," arXiv:1002.5012 [hep-ex].

[38] J. Knopf, Development, characterization and operation of the DCDB, the front-end readout chip for the pixel vertex detector of the future BELLE-II experiment. $\mathrm{PhD}$ thesis, Heidelberg Universität, 2011.

[39] M. Battaglia, "Vertex tracking at a future linear collider," Nuclear Instruments and Methods in Physics Research Section A: Accelerators, Spectrometers, Detectors and Associated Equipment 650 no. 1, (2011) 55 - 58. International Workshop on Semiconductor Pixel Detectors for Particles and Imaging 2010.

[40] J. Baudot et al., "First test results Of MIMOSA-26, a fast CMOS sensor with integrated zero suppression and digitized output," in Nuclear Science Symposium Conference Record (NSS/MIC), 2009 IEEE, pp. 1169-1173. Oct, 2009.

[41] J. Behr, "Test Beam Measurements with the EUDET Pixel Telescope," EUDET-Report-2010-01 (2010) .

[42] L. Andricek et al., "Intrinsic resolutions of DEPFET detector prototypes measured at beam tests," Nucl.Instrum.Meth. A638 (2011) 24-32

[43] M. Lemarenko et al., "Test results of the Data Handling Processor for the DEPFET Pixel Vertex Detector," JINST 8 no. 01, (2013).

[44] GEANT4 Collaboration, S. Agostinelli et al., "Geant4: A simulation toolkit," Nucl.Instrum.Meth. A506 (2003) 250-303.

[45] R. Frühwirth, "Application of Kalman filtering to track and vertex fitting," Nucl.Instrum.Meth. A262 no. 2-3, (1987) 444-450.

[46] F. Gaede, "Marlin and LCCD: Software tools for the ILC," Nucl.Instrum.Meth. A559 (2006) 177-180.

[47] F. Gaede and J. Engels, "Marlin et al - A Software Framework for ILC and detector R\&D," EUDET-Report-2007-11 (2007) .

[48] F. Gaede et al., "LCIO: A Persistency framework for linear collider simulation studies," arXiv:physics/0306114 [physics],

[49] L. D. Landau, “On the energy loss of fast particles by ionization,” J. Phys. 8 no. 4, (1944) 201-205.

[50] K. Lassila-Perini and L. Urban, "Energy loss in thin layers in GEANT," Nucl.Instrum.Meth. A362 no. 2-3, (1995) 416-422. 
[51] V. N. Ivanchenko et al., "Geant4 models for simulation of multiple scattering," JINST: Conference Series 219 no. 3, (2010).

[52] K. Gärtner and R. Richter, "DEPFET sensor design using an experimental 3d device simulator," Nucl.Instrum.Meth. A568 no. 1, (2006) 12 - 17 .

[53] R. Richter. Private communications, 2011.

[54] G. Lutz, Semiconductor Radiation Detectors: Device Physics. Accelerator Physics Series. U.S. Government Printing Office, 1999.

[55] F. Wilk, Study of Straggling and Extreme Cases of Energy Deposition in Micron Scale Silicon Volumes using the DEPFET Detector. Georg-August-Universität Göttingen II.Physik-UniGö-BSc-2012/05, 2012.

[56] R. Turchetta, "Spatial resolution of silicon microstrip detectors," Nucl.Instrum.Meth. A335 (1993) $44-58$

[57] B. Schwenker et al., "Physical limitations to the spatial resolution of solid-state detectors," arXiv:1404.4535 [physics.ins-det].

[58] T. Behnke et al., “Test Beams at DESY,” EUDET-Memo-2007-11 (2007) .

[59] E. Corrin, "EUDAQ software user manual,” EUDET-Memo-2010-01 (2010) .

[60] D. Haas, "The EUDET pixel telescope data acquisition system," in Real Time Conference, 2009. RT'09. 16th IEEE-NPSS, pp. 366-371, IEEE. 2009.

[61] R. Brun and F. Rademakers, "ROOT - An object oriented data analysis framework," Nucl.Instrum.Meth. A389 no. 1-2, (1997) 81-86.

[62] L. Rossi, Pixel Detectors: From Fundamentals to Applications. Particle Acceleration and Detection. Springer, 2006.

[63] H. Gjersdal et al., "Optimizing track reconstruction by simultaneous estimation of material and resolutions," JINST 8 no. 01, (2013) .

[64] V. Karimaki et al., "Straight Line Fit for Pixel and Strip Detectors with Arbitrary Plane Orientations," CMS-NOTE-1999-041 (1999) .

[65] D. N. Brown, E. A. Charles, and D. A. Roberts, "The BaBar Track Fitting Algorithm," in Proc. Computing in High Energy Physics Conference, Padova. 2000.

[66] R. Frühwirth et al., "The DELPHI forward track fit Track fitting with outlier rejection," Nucl.Instrum.Meth. A334 no. 2-3, (1993) 528 - 536.

[67] U. Stolzenberg, Measurement of the radiation length X/XO by using the EUDET tracking telescope. Georg-August-Universität Göttingen II.Physik-UniGö-MSc-2013/08, 2013.

[68] P. Wieduwilt, Position resolved measurement of the radiaiton length for DEPFET pixel modules. Georg-August-Universität Göttingen II.Physik-UniGö-BSc-2014/03, 2013. 


\section{Bibliography}

[69] V. Karimaki et al., "Sensor Alignment by Tracks,"arXiv:physics/0306034.

[70] D. Brown et al., "Local alignment of the BaBar Silicon Vertex Tracking detector," Nucl.Instrum.Meth. A603 no. 3, (2009) 467 - 484.

[71] E. Widl, Global Alignment of the CMS Tracker. PhD thesis, Technische Universität Wien, 2008.

[72] S. Rummel, "The power supply system for the DEPFET pixel detector at BELLE II," Nucl.Instrum.Meth. A699 no. 0, (2013) 51 - 55

[73] M. Koch, Development of a test environment for the characterization of the current digitizer chip DCD2 and the DEPFET pixel system for the Belle II experiment at SuperKEKB. PhD thesis, Bonn Universität, 2011.

[74] S. Furletov, "A system for characterization of DEPFET silicon pixel matrices and test beam results ,"Nucl.Instrum.Meth. A628 no. 1, (2011) 221 - 225

[75] J. Furletova and L. Reuen, "JRA1-The DEPFET sensor as the first fully integrated DUT in the EUDET pixel telescope: The PS test beam 2008," Eudet-Memo-2008-34 (2009) .

[76] J. Furletova, "DEPFET SPS beam test using the EUDET telescope," Eudet-Memo-2009-19 (2009) .

[77] T. Obermann et al., "Implementation of a configurable FE-I4 trigger plane for the AIDA telescope," JINST 9 (2014) C03035.

[78] P. G. Kaminski, Square root filtering and smoothing for discrete processes. Department of Aeronautics and Astronautics, Stanford University, 1971.

[79] E. Wolin and L. Ho, "Covariance matrices for track fitting with the Kalman filter ," Nucl.Instrum.Meth. A329 no. 3, (1993) 493 - 500 . 


\section{Benjamin Schwenker}

\section{Curriculum Vitae}

\section{Personal Details}

Name Benjamin Schwenker.

Date of Birth

Place of Birth

Email benjamin.schwenker@phys.uni-goettingen.de.

Nationality

German.

\section{Education}

2009-2014 PhD Physics, Georg-August-Universität Göttingen, Göttingen, Germany.

2004-2008 Diploma Physics, Georg-August-Universität Göttingen, Göttingen, Germany.

2002-2004 Intermediate Diploma Physics, Universität Rostock, Rostock, Germany.

1994-2002 Abitur, Gymnasium Bondenwald, Hamburg, Germany.

\section{Professional Experience}

2012current

2012 Talk at IEEE NSS 2012, Anaheim, California, USA.

2010 42. Herbstschule für Hochenergiephysik Maria Laach. 
2008 DESY Summer Student Program 2008, Hamburg, Germany.

\section{Languages}

German Native

English Fluent

Toefl test successfully finished 2004

\section{List of Publications}

- L. Andricek et al., Intrinsic resolutions of DEPFET detector prototypes measured at beam tests, Nucl.Instrum.Meth. A638, 2011

- B. Schwenker et al., Physical limitations to the spatial resolution of solid-state detectors, arXiv:1404.4535 [physics.ins-det]

- Z. Drasal, K. Prothmann, and B. Schwenker, Silicon simulation code for Belle II and ILC, PoS VERTEX2011 (2011) 027

- Alonso et al., DEPFET active pixel detectors for a future linear $e^{+} e^{-}$collider, IEEE TNS, vol.60, no.2, 2013

- I. Peric et al., High-voltage pixel detectors in commercial CMOS technologies for ATLAS, CLIC and Mu3e experiments, Nucl.Instrum.Meth. A731, 2013 